\title{
Uso de Modeios de Estratégia de Tipo II em Confiabilidade de Software
}

\author{
Karin Storani
}

\section{Orientador: Prof. Dr. Jorge Alberto Achcar}

Dissertação apresentada ao Instituto de Ciências Matemáticas de São Carlos-USP, como parte dos requisitos para a obtenção do título de Mestre em Ciências - Área de Ciências de Computação e Matemática Computacional.

USP-São Carlos

Maio/1997 
Em especial, ao Mario Eduardo (Dú) e aos meus pais João e Marly. 


\section{Agradecimentos}

A Deus por mais essa realização.

Ao Prof. Jorge Alberto Achcar, por sua orientação segura no decorrer do presente trabalho, incentivo a pesquisa e, principalmente, pelo seu exemplo de dedicação e disciplina ao trabalho.

Aos Prof. Josemar Rodrigues e Carlos Alberto Ribeiro Diniz pelas valiosas sugestões no exame de qualificação.

Em especial ao Prof. Marinho pela sempre boa vontade em ajudar e pelas valiosas sugestões para redação deste trabalho.

Aos colegas e Funcionários do ICMSC-USP.

A FAPESP pelo apoio financeiro.

Ao Mario Eduardo, por estar sempre presente em todos os momentos da minha vida, sendo o porto seguro nos momentos mais dificeis e sempre comemorando comigo as nossas conquistas.

À minha familia, que com paciência sempre compreendeu minhas ausências. Em especial, aos meus pais João e Marly por acreditarem em mim e me apoiarem.

Aos amigos Danieli, Daniela e Luciano pelo apoio e principamente, pela amizade no decorrer deste trabalho.

Ao Sr. Andrade e D.Alice, que me acolheram como uma filha e sempre me incentivaram muito. Também à $\mathrm{D}$. Antonieta, pela amizade e apoio nos momentos dificeis. 
Ao Prof. Jose Carlos Fogo pelas valiosas sugestões dadas à esse trabalho.

Enfim, a todos que direta ou indiretamente contribuíram para a realização deste trabalho. 


\section{Terminologia e Notação}

Variáveis aleatórias são representadas por letras maiúsculas e suas realizações por minúsculas.

A função densidade de probabilidade e função distribuição de uma variável aleatória é representada por $f(\cdot)$ e $\mathrm{F}(\cdot)$ respectivamente, as densidades a priori por $\pi(\cdot)$, as densidades a posteriori por $\pi(\cdot \backslash \mathrm{x})$.

Para simplificação de notação as funções de verossimilhança dos parâmetros envolvidos são representada por $\mathrm{L}(\cdot \mid \underset{\sim}{\mathrm{x}})=\mathrm{L}(\cdot)$.

Letras gregas indicam os parâmetros dos modelos.

Em geral, constantes são representadas por letras minúsculas.

Vetores são representados por letras sublinhadas.

O conjunto dos números reais positivos é denotado por $\mathfrak{R}^{+}$.

As seguintes abreviações são utilizadas:

EMV Estimador de Máxima Verossimilhança

i.i.d. independente e identicamente distribuída 


\section{Abstract}

In this dissertation, we explore software reliability models based on homogeneous and nonhomogeneous Poisson process to model software failure data. To model the epochs of the software failures, we choose the family of order statistics models introduced by Yang (1994). We propose some generalization of these models considering the Gaussian inverse distribution, to model the mean value function of the nonhomogeneous Poisson process. The proposed methods to get inferences on the parameters of interest are given by the Bayesian approach. We also explore the use of Metropolis-with-Gibbs algorithms to obtain the posterior summaries of interest. To verify the adequability of the proposed models, we develop some diagnostic Bayesian methods. We choose the best model, based on the values of ordinated predictives. The methodology presented in this work is illustrated with the data sets introduced by Jelinski and Moranda (1972) and Goel (1985). 


\section{Resumo}

Nesta dissertação de mestrado, exploramos os modelos estatísticos de confiabilidade de software que utilizam os processos de Poisson homogêneo e não homogêneo para modelagem dos dados de falhas. Para modelar os instantes de falhas, escolhemos a classe de modelos de estatísticas de ordem proposta por Yang (1994). Propomos uma extensão desses modelos, considerando a distribuição gaussiana inversa, para modelar a função de valor médio dos processos de Poisson não homogêneo. Os métodos considerados para fazer inferências para os parâmetros de interesse são os métodos Bayesianos. Exploramos, ainda, o uso de algoritmos de Metropolis com etapas Gibbs para desenvolver a inferência Bayesiana. Tendo em vista a verificação das suposições dos modelos de estatísticas de ordem, desenvolvemos e incorporamos algumas técnicas Bayesianas de diagnóstico. Baseamos a seleção de modelos nos valores de predição ordenados. A metodologia desenvolvida neste trabalho é exemplificada com conjuntos de dados introduzidos por Jelinski e Moranda (1972) e Goel (1985). 


\section{Conteúdo}

Capítulo 1 Introdução.

1.1 Confiabilidade de Software.

1.2 Processos de Poisson em Confiabilidade de Software.

1.2.1 Modelos de Confiabilidade de Software com Processos de Poisson Não Homogêneo. 5

1.3 Motivações e Objetivos................................................................... 10

1.4 Organização do Trabalho.................................................................11

Capítulo 2 Análise Clássica para Alguns Modelos de Estratégia Tipo II

2.1 Análise Clássica do Modelo de Moranda (1975)............................14

2.1.1 Formulação do Modelo de Moranda....................................15

2.1.2 Função de Verossimilhança...............................................16

2.1.3 Estimadores de Máxima Verossimilhança...........................16

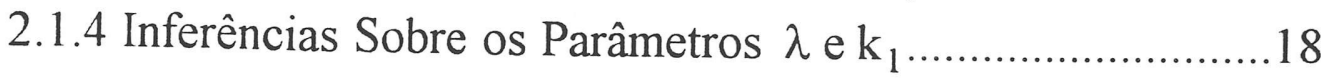

2.2 Análise Clássica da Generalização do Modelo de Moranda............20 (1975)

2.2.1 Motivação para a Generalização do Modelo de Moranda

2.2.2 Função de Verossimilhança...............................................22

2.2.3 Estimadores de Máxima Verossimilhança..........................22

2.2.4 Inferências Sobre os Parâmetros $\lambda_{\mathrm{a}}, \mathrm{k}_{1}$ e $\mathrm{k}_{2} \ldots \ldots \ldots \ldots \ldots \ldots \ldots . . . .24$

Capítulo 3 Análise Bayesiana para Alguns Modelos de Estratégia TipoII. 28

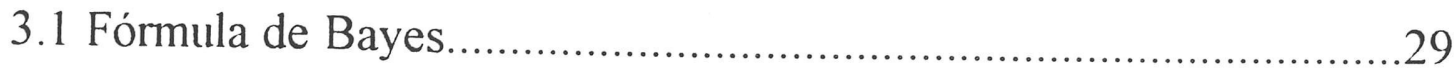

3.2 Densidades a Priori e a Posteriori...................................................29

3.3 Algoritmos de Simulação de Monte Carlo com Cadeia de Markov.31

3.3.1 Convergência dos Algoritmos Amostrador de Gibbs e Metropolis-Hastings.

3.4 Análise Bayesiana para o Modelo de Moranda Considerando Densidades a Priori Informativas. 
3.4.2 Densidades a Posteriori Conjunta para $\lambda \mathrm{e}_{1}$ 33

3.4.2.1 Densidade Condicional de $\lambda$ para o Algoritmo Amostrador de Gibbs. 34

3.4.2.2 Densidades Condicionais de $\mathrm{k}_{1}$ para o Algoritmo Amostrador de Gibbs. 34

3.4.3 Densidade Preditivas e Momentos a Posteriori 36 3.5 Análise Bayesiana da Generalização do Modelo de Moranda Considerando Densidades a Priori Informativas 37

3.5.1 Densidades a Priori Informativas para $\lambda_{\mathrm{a}}, \mathrm{k}_{1}$ e $\mathrm{k}_{2} \ldots \ldots \ldots . . .38$ 3.5.2 Densidades a Posteriori Conjunto para $\lambda_{\mathrm{a}}, \mathrm{k}_{1}$ e $\mathrm{k}_{2} \ldots \ldots \ldots . .39$

3.5.2.1 Densidade Condicional de $\lambda_{\mathrm{a}}$ para o algoritmo Amostrador de Gibbs. 39

3.5.2.2 Densidade Condicional de $\mathrm{k}_{1}$ para o algoritmo de Gibbs

3.5.2.3 Densidade Condicional de $\mathrm{k}_{2}$ para o algoritmo de Gibbs 40

3.5.3 Momentos a Posteriori e Densidade Preditiva 41 Capítulo 4. Processos de Poisson Não Homogêneos:Um Enfoque Bayesiano. 42

4.1 Modelos de Confiabilidade de Software com Processos de Poisson não Homogêneos.

4.1.1 Modelos de Estatísticas de Ordem.....................................43

4.1.1.1 Função de Verossimilhança..................................44

4.1.1.2 Estatísticas de Ordem Genéricas e PPNH-I.........46

4.1.2 Modelo de Tempo Dependente e Detecção de Erro (Goel e Okumoto, 1979). 48

4.1.3 Modelo de Goel (1983) ..................................................49

4.1.4 Modelo de Confiabilidade de Software Tipo Gama..........50

4.1.5 Modelo de Confiabilidade de Software com Função de Valor Médio Tipo Gama Generalizada.

4.1.6 Modelo de Confiabilidade de Software com Função de Valor Médio Tipo Log Normal..... 
4.1.7 Modelo de Confiabilidade de Software com Função de Valor Médio Tipo Gaussiana Inversa.

4.1.7.1 Motivação para a Utilização da Distribuição

Gaussiana Inversa em Confiabilidade de Software. .53

4.1.7.2 Taxa de Falha. .54

Distribuição

4.1.7.3 Função Densidade de Probabilidade e Função Gaussiana Inversa dos PPNH-I

4.1.7.4 Função de Valor Médio e Taxa de Falha Tipo 4.2 Inferência Bayesiana para os Modelos de Confiabilidade de Software com Processos com Processos de Poisson não Homogêneo. 56

4.2.1 Análise Bayesiana do Modelo de Estatísticas de Ordem Gama Generalizada (Achcar, Dey e Niverthy, 1996)

4.2.1.1 Função de Verossimilhança.

4.2.1.2 Densidades a Priori para os Parâmetros $\theta, \alpha, \beta$ e $k$ .58

4.2.1.3 Densidades a Posteriori Conjunta para $\theta, \alpha, \beta$ e $k$

4.2.1.4 Densidades Condicionais de $\theta, \alpha, \beta$ e $k$ para o Algoritmo Amostrador de Gibbs.

4.2.2 Análise Bayesiana do Modelo de Estatísticas de Ordem Gama Generalizada (Achcar, Dey e Niverthy, 1996) Considerando a Inclusão da Variável Latente $\left(\mathrm{N}^{\prime}=\mathrm{N}-\mathrm{n}\right)$. .64

4.2.2.1 Função de Verossimilhança.................................65 $\mathrm{N}^{\prime}, \theta, \alpha, \beta$ e $k$

4.2.2.2 Densidades a Priori para os Parâmetros

4.2.2.3 Densidades a Posteriori Conjunta para os Parâmetros $\mathrm{N}^{\prime}, \theta, \alpha, \beta$ e $k$ 66

4.2.2.4 Densidades Condicionais para $\mathrm{N}^{\prime}, \theta, \alpha, \beta$ e $k$ para o Algoritmo Amostrador de Gibbs.

4.2.3 Análise Bayesiana do Modelo de Goel (1983) Considerando a Inclusão da Variável Latente .68 
4.2.3.1 Densidade a Posteriori Conjunta.

algoritmo de Gibbs.

4.2.3.2 Densidades Condicionais para $\mathrm{N}^{\prime}, \theta, \alpha$ e $\beta$ para o

4.2.4 Análise Bayesiana para o Modelo de Estatísticas de ordem Gama Considerando a Inclusão da Variável Latente $\left(\mathrm{N}^{\prime}=\mathrm{N}-\mathrm{n}\right)$.

4.2.4.1 Densidade a Posteriori Conjunta de $\mathrm{N}^{\prime} \theta, \beta$ e $k$ para o algoritmo de Gibbs. algoritmo de Gibbs

4.2.4.2 Densidades Condicionais para $\mathrm{N}^{\prime} \theta, \beta$ e $k$ para o

4.2.5 Análise Bayesiana para o Modelo de Goel e Okumoto (1979) Considerando a Inclusão da Variável Latente $\left(N^{\prime}=N-n\right) \ldots \ldots . . .71$

4.2.5.1 Densidades a Posteriori Conjunta de $N^{\prime} \theta$ e $\beta \ldots .71$ algoritmo de Gibbs

4.2.5.2 Densidades Condicionais para $\mathrm{N}^{\prime} \theta$ e $\beta$ para o

4.2.6 Análise Bayesiana do Modelo de Estatísticas de Ordem Log-Normal Considerando a Inclusão da Variável Latente $\left(\mathrm{N}^{\prime}=\mathrm{N}-\mathrm{n}\right) 72$

4.2.6.1 Função de Verossimilhança..................................72

4.2.6.2 Densidades a Priori para $\mathrm{N}^{\prime}, \theta, \mu, \sigma \ldots \ldots \ldots \ldots \ldots \ldots . . .73$

4.2.6.3 Densidades a Posteriori Conjunta para $\mathrm{N}^{\prime}, \theta, \mu, \sigma$

4.2.6.4 Densidades Condicionais para $\mathrm{N}^{\prime}, \theta, \mu, \sigma$ para o Algoritmo Amostrador de Gibbs

4.2.7 Análise Bayesiana do Modelo de Estatísticas de Ordem Gaussiana Inversa (Achcar e Storani, 1996) Considerando a Inclusão da Variável Latente $\left(\mathrm{N}^{\prime}=\mathrm{N}-\mathrm{n}\right)$.

4.2.7.1 Função de Verossimilhança...................................75

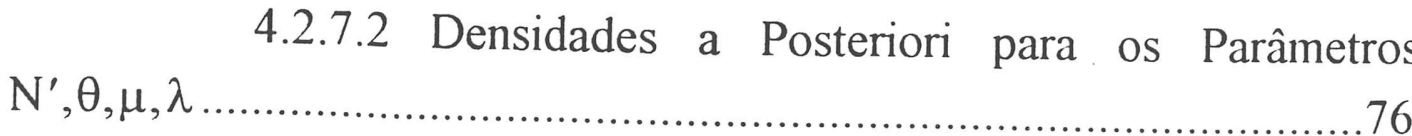

4.2.7.3 Densidades a Posteriori Conjunta de $N^{\prime}, \theta, \mu, \lambda . .76$

4.2.7.4 Densidades Condicionais de $\mathrm{N}^{\prime}, \theta, \mu, \lambda$ para o algoritmo Amostrador de Gibbs .77

Capítulo 5. Discriminação dos Modelos de Confiabilidade de Software. 
5.1 Seleção dos Modelos que Utilizam Processos de Poisson Homogêneo

5.2 Seleção dos Modelos de Confiabilidade de Software que Utilizam os Processos de Poisson não Homogêneo. .86

Capítulo 6. Exemplos de Aplicações......................................................91

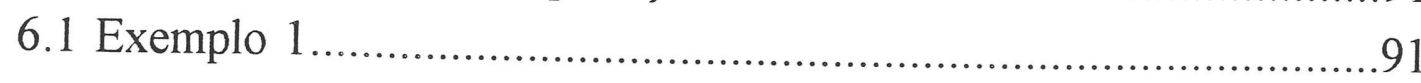

6.2 Exemplo 2

Apêndice A

Apêndice A.1 Amostrador de Gibbs

Apêndice A.2 Algoritmo de Metropolis-Hastings.

Apêndice A.3 Critério de Gelman e Rubin, 1992

140

Apêndice B Programa de Metropolis Hastings e Amostrador de Gibbs para o Modelo de Estatísticas de Ordem Gaussiana Inversa.

Bibliografia. 


\section{Capítulo 1}

\section{INTRODUÇÃO}

O software é o grande negócio de hoje e do futuro, com projeção de faturamento de um trilhão de dólares para o ano 2000 (Weber, Luca e Rocha, 97). O aumento considerável da oferta e da evolução de pacotes de software de sistemas dificulta a seleção desses pacotes, pois é cada vez maior o número de opções no mercado que aparentemente podem solucionar um problema específico da empresa nos seus diversos níveis de decisão.

Um critério de seleção que usualmente uma empresa deve levar em conta, ao selecionar um produto que deseja comprar, é a relação custo benefício que o produto trará à empresa. Dentro desse critério, um dos fatores predominantes é a qualidade do software.

A ISO/IEC $9126^{1}$ considera a qualidade de software através de 6 características: (1) Confiabilidade: tolerância às falhas; (2) Funcionabilidade: funções oferecidas ao usuário que atendem às suas necessidades (exigências); (3) Usabilidade: facilidade de uso; (4) Desempenho: desempenho do software em relação a tempo e recursos; (5) Manutenibilidade: facilidade de modificar o software e (6) Portabilidade: facilidade de mudar de ambiente.

Neste trabalho, estamos analisando modelos estatísticos para avaliar o item confiabilidade de software, fornecendo uma visão geral dos principais problemas (e de suas causas prováveis), tendo em vista a relevância da área de tolerâncias a falhas, tanto do ponto de vista técnico quanto econômico.

${ }^{1}$ ISO/IEC 9126 é a garantia de qualidade total do software. 


\subsection{Confiabilidade de Software}

Musa e Okumoto (1984) definem a confiabilidade de software como "probabilidade de um programa computacional estar livre de erros de operação, durante um período específico de tempo".

Vários modelos probabilísticos e técnicas estatísticas têm sido utilizados por pesquisadores da área de computação, para avaliar a performance (execução) do software, o qual está sujeito a falhas devido à presença de erros.

Erros ou "bugs" são defeitos de projeto que não foram detectados em fase de testes e que, sob certas condições, podem causar ou não uma ou mais falhas. A falha de um software é um desvio do comportamento exigido em relação ao sistema, causada por erros ou "bugs" existentes no programa, por vírus ou pelo uso impróprio do software pelo usuário.

A confiabilidade é uma função que expressa a probabilidade de que um sistema funcione corretamente no intervalo de tempo $(0, \mathrm{t}]$. Durante o estágio de teste, os erros são detectados e corrigidos, aperfeiçoando (ou melhorando) a confiabilidade do software. Espera-se, então, que o número de falhas no intervalo $(0, t]$ decresça ou que os tempos entre as falhas sejam crescentes.

Um modelo que descreve tal comportamento das falhas é chamado de modelo de confiabilidade crescente de software.

A literatura em estatística e em engenharia, apresenta vários modelos para modelar a confiabilidade do software (ver por exemplo, Mazzuchi e Singpurwalla, 1988 ou Singpurwalla e Wilson, 1994).

Duas estratégias de modelagem amplamente utilizadas são:

(i) Estratégia de Tipo I: Modela os tempos entre falhas sucessivas do software.

(ii) Estratégia de Tipo II: Modela o número de falhas do software até um determinado tempo (usual: tempo de CPU). 
A estratégia de modelagem de tipo-I pode ser classificada em:

(i.1) Estratégia de tipo I-1, quando o modelo é construído a partir de considerações de taxas de falhas do software.

(i.2) Estratégia de tipo I-2, quando o modelo é construído a partir de uma relação estocástica entre os tempos de falhas sucessivas.

Os modelos de confiabilidade de software de estratégia de tipo II são classificados conforme a especificação da distribuição da quantidade de falha (número de falhas observadas no intervalo de tempo $(0, \mathrm{t}]$ ).

Os Processos de Poisson homogêneo, não homogêneo e binomial são os mais importantes para modelar o número de falhas observadas no intervalo de tempo $(0, \mathrm{t}]$ (ver Musa, Iannino e Okumoto, 1987).

Neste trabalho, consideramos os modelos de Estratégia de Tipo II, que utilizam os processos de Poisson homogêneo e não homogêneo.

Para os modelos com processo de Poisson homogêneo, foi feito um estudo comparativo das inferências obtidas para os parâmetros de interesse, usando-se métodos estatísticos clássicos e Bayesianos.

Os modelos que utilizam processo de Poisson não homogêneo tornam-se modelos muito complexos e, geralmente, apresentam vários parâmetros $\beta=\left\{\beta_{1}, \beta_{2}, \ldots, \beta_{n}\right\}$. Devido a essa característica, são várias as dificuldades encontradas para se fazer inferências sobre $\beta$ principalmente quando utilizamos os métodos clássicos de inferência, tais como o método de máxima verossimilhança e mínimos quadrados.

Assim, para a obtenção de sumários de interesse dos modelos de confiabilidade, com processo de Poisson não homogêneo, utilizamos os métodos Bayesianos e algoritmos computacionais, como amostrador de Gibbs e Metropolis-Hastings (Gelfand e Smith, 1990). Estes amostradores são técnicas que permitem a geração de variáveis aleatórias de uma distribuição (marginal) indiretamente, isto é, sem necessidade do cálculo da densidade marginal de $\beta$. 


\subsection{Processos de Poisson em Confiabilidade de Software}

Um modelo com processo pontual estocástico pode ser usado para contagem do número de falhas num dado intervalo de tempo. O processo de Poisson não homogêneo, que é um caso especial de processos pontuais estocásticos, é um processo adequado para descrever o comportamento estocástico do número de falhas de um software.

Seja $M(t)$ o número de falhas do software observadas durante um intervalo de tempo $(0, t]$; então $M(t)$ pode ser modelado por um processo de Poisson não homogêneo baseado nas seguintes suposições:

1) Não existem falhas de software no tempo $t=0$, isto é, $M(0)=0$ com probabilidade 1 .

2) $O$ processo tem incrementos independentes, ou seja, o número de falhas observadas durante o intervalo ( $t, t+\Delta t$ ], isto é, $M(t+\Delta t)-M(t)$, é independente dos valores passados. Observe que esta suposição implica propriedades de Markov, ou seja, o futuro $M(t+\Delta t)$ do processo depende apenas do estado presente $\mathrm{M}(\mathrm{t})$ e independe do passado $\mathrm{M}(\mathrm{x})$ para $\mathrm{x}<\mathrm{t}$.

3) A probabilidade de que uma falha ocorra durante o intervalo $(t, t+\Delta t]$ e $\lambda(t) \Delta t+o(\Delta t)$, onde $\lambda(t)$ é a intensidade de falha do processo. Observe que a função $\mathrm{o}(\mathrm{t})$ é definida como,

$$
\lim _{t \rightarrow 0} \frac{o(\Delta t)}{\Delta t}=0
$$

4) A probabilidade de que mais de uma falha ocorra durante o intervalo ( $t$, $t+\Delta t]$ é $o(t)$. 
Portanto, $\mathrm{M}(\mathrm{t})$ é uma variável aleatória com distribuição de Poisson com média e variância $\mathrm{m}(\mathrm{t})$, a qual é chamada função de valor médio do processo, na forma,

$$
\mathrm{P}\{\mathrm{M}(\mathrm{t})=\mathrm{n}\}=\frac{[\mathrm{m}(\mathrm{t})]^{\mathrm{n}}}{\mathrm{n} !} \mathrm{e}^{-\mathrm{m}(\mathrm{t})} \text { onde } \mathrm{n}=0,1,2, \ldots
$$

onde,

$$
m(t)=\int_{0}^{t} \lambda(x) d x
$$

Se $\lambda(t)$ é uma constante, ou seja, $m(t)$ é linear, então, $M(t)$ é chamado processo de Poisson homogêneo, caso contrário, $M(t)$ é um processo de Poisson não homogêneo.

$\mathrm{O}$ processo $\mathrm{M}(\mathrm{t})$ pode ser particularizado por funções específicas de valor médio $m(t)$, ou por funções intensidades de falhas, obtidas pela derivação de $m(t)$ em relação a $t$, ou seja, $\lambda(t)=d m(t) / d t$.

\subsubsection{Modelos de Confiabilidade de Software com Processos de Poisson Não Homogêneo}

Os modelos de confiabilidade de software com processo de Poisson não homogêneo são classificados conforme o comportamento limite da função de valor médio: (1) modelos com número de falhas finito $\left(\lim _{t \rightarrow \infty} \mathrm{m}(\mathrm{t})<\infty\right)$, denotados por PPNH-I; (2) modelos com número de falhas infinito (ou seja $m(t) \rightarrow \infty$ com $t \rightarrow \infty)$, denotados por PPNH-II.

Yang (1994) propõe uma teoria unificada para os modelos com número de falhas finito e infinito, modelando as falhas do software por duas classes diferentes de modelos: modelos de estatísticas de ordem, e modelos de estatísticas de valores observados. 
Nos modelos de estatísticas de ordem, assume-se um número desconhecido de erros $\mathrm{N}$, no início do teste do software. Os tempos observados de falhas, nesse modelo, são as $\mathrm{n}$ primeiras estatísticas de ordem i.i.d. de $\mathrm{N}$ com função densidade $f$ limitada em $\mathfrak{R}^{+}$.

Os modelos de estatísticas de ordem têm aplicação limitada para o teste onde novos erros são introduzidos no software em cada reparo feito. Durante o teste do software em cada reparo de erros, novos erros são introduzidos no software; nessas situações, utiliza-se o modelo de estatísticas de valores observados, onde se assume que os tempos de falhas são as estatísticas observadas i.i.d., com função densidade $f$, limitada em $\mathfrak{R}^{+}$.

O processo de contagem das estatísticas de ordem e das estatísticas observadas, num dado intervalo de tempo, é modelado como um processo de Poisson não homogêneo (ver Yang, 1994).

Os modelos de estatísticas de ordem assumem que $\mathrm{N}$ é uma variável aleatória, com distribuição de Poisson com média $\theta$. Então, pode-se mostrar que $\mathrm{M}(\mathrm{t})$ é um processo PPNH-I, com função de valor médio dada por,

$$
\mathrm{m}(\mathrm{t})=\theta \mathrm{F}(\mathrm{t})
$$

onde $\mathrm{F}$ é a função distribuição, com densidade $f$.

A função intensidade pode ser obtida derivando $m(t)$ com respeito a $t$. Assim, temos,

$$
\lambda(\mathrm{t})=\theta f(\mathrm{t})
$$

Supondo que $f$ é a distribuição exponencial em (1.2.3), temos o processo de Goel e Okumoto(1979), o qual é chamado de modelo de estatísticas de ordem exponencial. Outros modelos de estatísticas de ordem podem ser considerados como, por exemplo, os modelos de estatísticas de ordem Pareto, Weibul e gama, onde $f$ de (1.2.3) é a distribuição Pareto, Weibul e gama respectivamente. 
Para os modelos de estatísticas de valores observados, pode-se mostrar que $\mathrm{M}(\mathrm{t})$ é um PPNH-II, com função de valor médio dada por,

$$
m_{\text {II }}(t)=-\ln (1-F(t))
$$

Portanto, os processos PPNH-I e PPNH-II são particularizados por funções distribuições $F(t)$ específicas em (1.2.2) e (1.2.4).

A seguir, apresentamos alguns exemplos de modelos de confiabilidade de software que utilizam processos de Poisson homogêneo e não homogêneo.

Exemplo 1.1 Moranda (1975) assume um modelo de confiabilidade de software, com um processo de Poisson homogêneo, para descrever o número de falhas em cada período sucessivo de tempo. Assim, considera uma função intensidade constante para um intervalo de tempo, mas formando uma seqüência decrescente na forma

$$
\lambda_{\mathrm{i}}=\lambda \mathrm{k}_{1}^{\mathrm{i}-1} \text { onde } \mathrm{i}=1,2,3, \ldots ; \lambda>0 \text { e } 0 \leq \mathrm{k}_{1} \leq 1
$$

onde $\lambda$ pode ser interpretado como o valor médio de falhas do software e $k_{1}$ como a probabilidade de ocorrer uma falha no i-ésimo intervalo de tempo. Esse modelo é formulado sob a argumentação de que a correção de erros que causam as primeiras falhas reduz a taxa de falha mais do que os erros que ocorrem mais tarde.

Exemplo1.2 Goel e Okumoto (1979) propõem um modelo de estatísticas de ordem exponencial para a confiabilidade de software com um processo de Poisson não homogêneo, com função distribuição $F(t)=1-e^{-\beta t}$ e função de valor médio $m(t)$ não-decrescente, dada por:

$$
m(t)=\theta\left(1-e^{-\beta t}\right)
$$


e função intensidade dada por,

$$
\lambda(t)=\frac{d m(t)}{d t}=\theta \beta e^{-\beta t}
$$

Nesse modelo, o número total de erros no software é uma variável aleatória com média $\theta$ e os tempos entre sucessivas falhas são dependentes e distribuídos exponencialmente. Para um dado número de falhas $\mathrm{w}_{0}$, o número total esperado de falhas será $\mathrm{w}_{0} / \beta$, onde $\beta$ corresponde ao fator de redução de erro.

Exemplo1.3 Experiências têm mostrado que, geralmente, a taxa de falhas no software cresce inicialmente antes de um eventual decréscimo; Goel (1983) sugere um processo não homogêneo $F(t)=1-e^{-\beta t^{\alpha}}$ (modelo de estatísticas de ordem Weibull), que é uma generalização do modelo de Goel e Okumoto (1979), com função de valor médio e função intensidade dadas por,

$$
m(t)=\theta\left(1-e^{-\beta t^{\alpha}}\right),
$$

e

$$
\lambda(t)=\frac{d m(t)}{d t}=\theta \beta \alpha t^{\alpha-1} e^{-\beta t^{\alpha}}
$$

onde $\theta$ é o número médio de erros no software e $\beta$ e $\alpha$ descrevem a qualidade do teste.

Exemplo 1.4. Achcar, Dey e Niverthy (1996) propõem um "super modelo" de confiabilidade de software com processo de Poisson não homogêneo, assumindo uma função de valor médio dada por

$$
m(t)=\theta I_{k}\left(\beta t^{\alpha}\right)
$$


onde $I_{k}$ é uma integral gama incompleta dada por

$$
I_{k}(s)=\frac{1}{\Gamma(k)} \int_{0}^{s} x^{k-1} e^{-x} d x
$$

e função intensidade dada por

$$
\lambda(\mathrm{t})=\frac{\mathrm{dm}(\mathrm{t})}{\mathrm{dt}}=\frac{1}{\Gamma(\mathrm{k})} \theta \beta^{\mathrm{k}} \alpha \mathrm{t}^{\alpha \mathrm{t}-1} \mathrm{e}^{-\beta \mathrm{t}^{\alpha}}
$$

onde $\theta, \alpha, \beta$ e k são os parâmetros dos modelos e $\Gamma(\mathrm{k})$ denota a função gama.

Exemplo 1.5 Achcar, Dey e Niverthy (1996) também consideram um modelo com processo de Poisson não homogêneo com função de valor médio dada por,

$$
\mathrm{m}(\mathrm{t})=\theta \Phi\left(\frac{\ln t-\mu}{\sigma^{2}}\right)
$$

onde $\Phi(\cdot)$ é uma função distribuição de uma normal padronizada (função distribuição log-normal em (1.2.2)).

Derivando (1.2.5), obtemos a intensidade de falha dada por,

$$
\lambda(t)=\frac{\theta}{t \sqrt{2 \pi \sigma}} \exp \left\{-\frac{(\ln t-\mu)^{2}}{2 \sigma^{2}}\right\},
$$

onde $\theta$ é o número médio de erros do software, e $\mu$ e $\sigma$ são os parâmetros do modelo. 


\subsection{Motivações e Objetivos}

$\mathrm{Na}$ prática, temos interesse em obter estimativas dos parâmetros dos modelos de confiabilidade de software com processos de Poisson homogêneo e não homogêneo $\theta$ e $\beta=\left\{\beta_{1}, \ldots, \beta_{n}\right\}$, ou funções dos mesmos, através dos dados de falhas.

A inferência para os modelos de confiabilidade de software tem sido baseada em métodos estatísticos clássicos usuais. Entretanto, Forman e Singpurwalla (1977) mostram que o estimador de máxima verossimilhança do parâmetro N do modelo de Jelinski e Moranda (1972) pode ser altamente induzido ao erro "viciado" e às vezes absurdo. Campodonico e Singpurwalla (1994) mostram que as funções de verossimilhança dos modelos de confiabilidade de software com processo de Poisson não homogêneo podem apresentar vários máximos locais, o que pode causar dificuldades computacionais, quando calculamos as estimativas dos parâmetros.

Meinhold e Singpurwalla (1985), Jewell (1985), Littlewood e Sofer (1987), Yang (1994), Kuo e Yang (1994), Achcar, Dey e Niverthy (1996) e Kuo, Lee, Chang e Yang (1996) argumentam em favor da inferência Bayesiana para os parâmetros dos modelos de confiabilidade de software.

O presente trabalho, inserido neste contexto, tem como objetivo principal apresentar uma análise Bayesiana para alguns dos modelos da confiabilidade de software de estratégia de Tipo II, que utilizam os processos de Poisson homogêneo e não homogêneo para modelagem dos dados de falhas. $O$ desenvolvimento da análise Bayesiana para esses modelos envolve o cálculo de uma integral multidimensional, uma vez que os modelos apresentam vários parâmetros. Assim a utilização de métodos computacionais torna-se necessária para a solução das integrais Bayesianas de interesse. Os métodos computacionais utilizados são os algoritmos computacionais amostrador de Gibbs e o de Metropolis-Hastings, devido à simplicidade de implementação dessas técnicas comparadas aos demais métodos numéricos de aproximação de integrais propostos na literatura, como por exemplo, o método de Laplace (ver Tierney e Kadane, 1986). 
Devido à diversidade de modelos de confiabilidade de software propostos na literatura, uma questão que surge é: "Qual modelo de confiabilidade é o mais adequado para um determinado conjunto de dados?". Sob o enfoque Bayesiano, essa questão pode ser respondida através de várias estratégias de discriminação propostas na literatura.

Abdel, Littlewood, Ghaly e Chan (1986) propõem o u-plot; ordenando os valores $u_{i}=P\left(T_{i}<t_{i}\right)$ e plotando os pontos $\left(u_{j}, j / c\right)$, onde c é o número total de falhas no software observadas no intervalo $(0, t]$. Algum vício na predição será destacado pelo desvio da linha unitária com inclinação na origem.

Mas considerando-se que o teste do software é feito em um tempo real, uma análise frequencial será apropriada. Assim, Dawid (1984) propõe para a seleção de modelos de confiabilidade de software o critério de verossimilhança frequencial. Singpurwalla e Soyer (1992), Yang (1994) e Achcar, Dey e Niverthy (1996) também utilizam a análise frequencial para comparação dos modelos de confiabilidade de software.

Neste trabalho, escolhemos o modelo que maximiza a densidade preditiva Bayesiana. Essa densidade preditiva é também o produto das preditivas condicionais frequenciais ordenadas, denotadas por PCPO. Consideramos a análise frequencial, pois o software é usualmente testado num tempo real. A seleção de modelos também pode ser baseada na soma de erros relativos. Assim o modelo escolhido minimiza a soma desses erros.

\subsection{Organização do Trabalho}

O presente trabalho está organizado em 7 capítulos. Este capítulo introdutório apresenta os modelos de confiabilidade considerados nesta dissertação e descreve os problemas de estimação a serem atingidos.

No Capítulo 2, são apresentados os modelos de confiabilidade de software com processo de Poisson homogêneo, dentre os quais, destacamos:

- Modelo de Moranda (1975), 
- Generalização do modelo de Moranda (Achcar, 1995).

Sob o enfoque da inferência clássica, são feitas inferências sobre os parâmetros de interesse para esses modelos.

No Capítulo 3, os modelos que utilizam processos de Poisson homogêneo, são analisados sob o enfoque Bayesiano. Densidades a priori informativas para os parâmetros de interesse são consideradas. Algoritmos computacionais como o amostrador de Gibbs e Metropolis-Hastings (ver Gelfand e Smith, 1990) são utilizados.

No Capítulo 4, são explorados os modelos de confiabilidade de software com processo de Poisson não homogêneo. Dentre os vários modelos desta classe, destacam-se:

- Modelo de estatísticas de ordem exponencial (ver Goel e Okumoto, 1979);

- Modelo de estatísticas de ordem Weibul (ver Goel, 1983);

- Modelo de estatísticas de ordem gama (ver por exemplo Ohba-Yamada, 1983);

- Modelo de estatísticas de ordem gama generalizada (ver Achcar, Dey e Niverthy, 1996);

- Modelo de estatísticas de ordem log-normal (ver Achcar, Dey e Niverthy, 1996).

Considerando a distribuição gaussiana inversa para modelar a função intensidade de falha dos processos de Poisson não homogêneos (ver Achcar e 
Storani, 1996), apresentamos ainda, neste capítulo, uma extensão dos modelos de estatísticas de ordem.

Esses modelos são analisados sob o enfoque Bayesiano. Densidades a priori informativas e não-informativas para os parâmetros de interesse são consideradas. Os métodos computacionais amostrador de Gibbs e MetropolisHastings são aplicados para implementar os cálculos das integrais Bayesianas de interesse. Para o amostrador de Gibbs, utilizamos a técnica de dados aumentados (ver Tanner e Wong, 1987), uma vez que essa técnica facilita a especificação das densidades condicionais dos parâmetros desconhecidos, dada uma variável latente (ver Yang, 1994).

No Capítulo 5, com finalidade de verificar qual ou quais modelos são adequados para fazer inferências sobre o software, são desenvolvidas técnicas de discriminação para os modelos introduzidos nos capítulos 2 e 4 . Densidades preditivas e o algoritmo amostrador de Gibbs com Metropolis são considerados.

Exemplos de aplicação das metodologias considerando os modelos que utilizam processos de Poisson homogêneo e não homogêneo, são vistos no Capítulo 6. Um primeiro conjunto de dados, introduzido por Goel (1985), é utilizado para fazer análise Bayesiana e clássica dos modelos que usam processo de Poisson homogêneo. Para fazer uma análise Bayesiana dos modelos que utilizam processos de Poisson não homogêneo, consideramos um segundo conjunto de dados, introduzido por Jelinski \& Moranda (1972). As conclusões e perspectivas futuras são apresentadas no Capítulo 7.

No apêndice A, são apresentados os algoritmos amostrador de Gibbs, Metropolis-Hastings e o critério de verificação formal de convergência de Gelman e Rubin (1992). No apêndice B, apresentamos o programa desenvolvido para o modelo de estatísticas de ordem gaussiana inversa. 


\section{Capítulo 2}

\section{ANÁliSE CLÁSSICA PARA ALGUNS MODELOS DE ESTRATÉGIA TIPO II}

Um problema comum da área estatística é fazer inferências sobre o parâmetro de interesse ou função do mesmo, com informações vindas de uma amostra selecionada da população.

Os procedimentos clássicos usuais para fazer uma análise estatística dos dados consistem basicamente em fazer inferências (construir testes de hipóteses, intervalos de confiança, previsões, etc) a partir da distribuição normal assintótica para os estimadores de máxima verossimilhança.

Neste capítulo, realizamos uma análise clássica para os modelos da estratégia de tipo II, onde o número de falhas observadas num intervalo de tempo $(0, \mathrm{t}]$ é modelado por um processo de Poisson homogêneo. Nesta dissertação, os modelos considerados são o modelo de Moranda (1975) e sua forma generalizada (ver Achcar, 1995).

\subsection{Análise Clássica do Modelo de Moranda (1975)}

Nesta seção, determinamos os estimadores de máxima verossimilhança para o modelo de Moranda e, a partir da normalidade assintótica desses estimadores, obtemos as inferências sobre os parâmetros do modelo. 


\subsubsection{Formulação do Modelo de Moranda}

Quando as falhas do software são dadas em termos do número de falhas que ocorrem em um período fixo de tempo, Moranda (1975) propõe um processo de Poisson homogêneo para descrever o número de falhas em cada período sucessivo de tempo.

Esse modelo supõe que a correção de erros que causam as primeiras falhas no sistema reduz a taxa de falha mais do que a correção de erros que ocorrem mais tarde. Para ilustrar esse fato, observamos a figura (2.1), que mostra a taxa de falhas do modelo de Moranda para os dados introduzidos por Goel (1985).

$$
\lambda_{\mathrm{i}}=\lambda \mathrm{k}_{1}^{\mathrm{i}-1}
$$

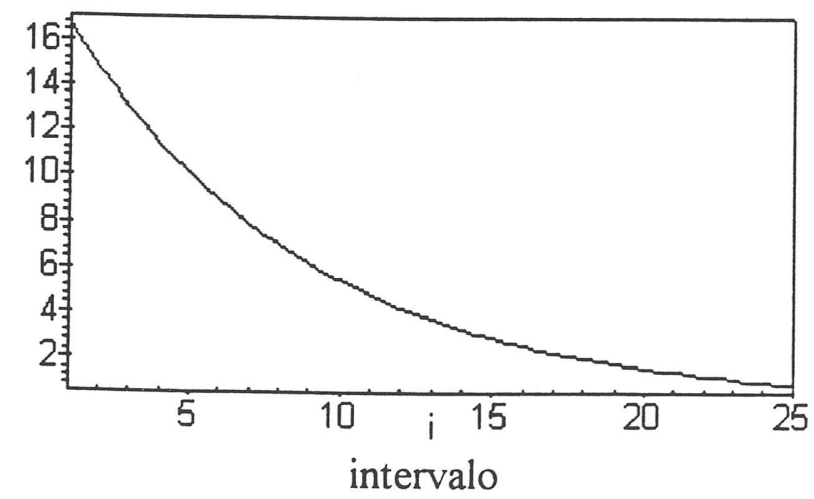

Figura 2.1: Taxa de falha do Modelo de Moranda para os dados introduzidos por Goel (1985).

Esse modelo assume que a taxa de falha é dada por,

$$
\lambda_{\mathrm{i}}=\lambda \mathrm{k}_{1}^{\mathrm{i}-1}, \text { onde } \lambda>0 \text { e } 0<\mathrm{k}_{1}<1, \mathrm{i}=1,2, \ldots \mathrm{n}
$$

O parâmetro $\lambda$ pode ser interpretado como o valor médio de falhas do software. $\mathrm{O}$ parâmetro $\mathrm{k}_{1}$ é a probabilidade de ocorrer uma falha no i-ésimo 
intervalo de tempo. Portanto, $\lambda_{\mathrm{i}}(\mathrm{i}=1,2, \ldots, \mathrm{n})$ é a taxa de falha no i-ésimo intervalo de tempo.

A função de probabilidade para o número de falhas $m_{i}$, no i-ésimo período de tempo, é dada por:

$$
\mathrm{P}\left(\mathrm{X}_{\mathrm{i}}=\mathrm{m}_{\mathrm{i}}\right)=\frac{\mathrm{e}^{-\lambda_{\mathrm{i}}} \lambda_{\mathrm{i}}^{\mathrm{m}_{\mathrm{i}}}}{\mathrm{m}_{\mathrm{i}} !} \text {, onde } \mathrm{m}_{\mathrm{i}}=0,1,2, \ldots
$$

onde $\lambda_{\mathrm{i}}$ é dado por (2.1.1).

\subsubsection{Função de Verossimilhança}

A função de verossimilhança resume toda a informação relevante que os dados fornecem a respeito dos parâmetros de interesse.

Para o modelo de Moranda com parâmetros $\lambda$ e $k_{1}$, considerando-se uma amostra aleatória de tamanho $n$, a função de verossimilhança é dada por

$$
L\left(\lambda, k_{1}\right)=\frac{e^{-\lambda \sum_{i=1}^{n} k_{1}^{i-1}}\left(\lambda^{\sum_{i=1}^{n} m_{i}} k_{1}^{\sum_{i=1}^{n} m_{i}(i-1)}\right)}{\prod_{i=1}^{n} m_{i} !} .
$$

\subsubsection{Estimadores de Máxima Verossimilhança}

Os estimadores de máxima verossimilhança são os valores dos parâmetros $\lambda$ e $\mathrm{k}_{1}$, que maximizam a função $\mathrm{L}\left(\lambda, \mathrm{k}_{1}\right)$, definida em (2.1.3). 
É comum determinarmos $\hat{\lambda}, \widehat{\mathrm{k}}_{1}$, a partir da função log-verossimilhança. Para o modelo em estudo, ignorando-se os termos que não dependem dos parâmetros, essa função é dada por:

$$
1\left(\lambda, \mathrm{k}_{1}\right) \propto-\lambda \sum_{\mathrm{i}=1}^{\mathrm{n}} \mathrm{k}_{1}^{\mathrm{i}-1}+\sum_{\mathrm{i}=1}^{\mathrm{n}} \mathrm{m}_{\mathrm{i}} \log \lambda+\sum_{\mathrm{i}=1}^{\mathrm{n}}(\mathrm{i}-1) \mathrm{m}_{\mathrm{i}} \log \mathrm{k}_{1}
$$

As primeiras derivadas da função de $1\left(\lambda, \mathrm{k}_{1}\right)$, com respeito a $\lambda$ e $\mathrm{k}_{1}$, são dadas por:

$$
\frac{\partial l\left(\lambda, \mathrm{k}_{1}\right)}{\partial \lambda}=-\sum_{\mathrm{i}=1}^{\mathrm{n}} \mathrm{k}_{1}^{\mathrm{i}-1}+\sum_{\mathrm{i}=1}^{\mathrm{n}} \frac{\mathrm{m}_{\mathrm{i}}}{\lambda}
$$

e

$$
\frac{\partial \mathrm{l}\left(\lambda, \mathrm{k}_{1}\right)}{\partial \mathrm{k}_{1}}=-\lambda \sum_{\mathrm{i}=1}^{\mathrm{n}} \mathrm{k}_{1}^{\mathrm{i}-2}(\mathrm{i}-1)+\sum_{\mathrm{i}=1}^{\mathrm{n}} \frac{\mathrm{m}_{\mathrm{i}}(\mathrm{i}-1)}{\mathrm{k}_{1}}
$$

Assim, os estimadores de máxima verossimilhança são obtidos através da resolução das equações:

$$
-\sum_{i=1}^{n} k_{1}^{i-1}+\sum_{i=1}^{n} \frac{m_{i}}{\lambda}=0
$$

e

$$
-\lambda \sum_{i=1}^{n} k_{1}^{i-1}(i-1)+\sum_{i=1}^{n} m_{i}(i-1)=0
$$


O estimador de máxima verossimilhança $\hat{\lambda}$ é dado por

$$
\hat{\lambda}=\frac{\sum_{i=1}^{n} m_{i}}{\sum_{i=0}^{n-1} \hat{k}_{1}^{i}},
$$

e o estimador de máxima verossimilhança de $\mathrm{k}_{1}$ é obtido através da resolução da equação,

$$
\frac{\sum_{i=1}^{n} m_{i}}{\sum_{i=0}^{n-1} i_{i+1}}=\frac{\sum_{i=0}^{n-1} k_{1}^{i}}{\sum_{i=1}^{n} k_{1}^{i-1}(i-1)} .
$$

Observe que para resolução da equação (2.1.5) torna-se necessária a utilização de um método numérico, como, por exemplo, o método iterativo de Newton-Raphson.

\subsubsection{Inferências sobre os Parâmetros $\lambda$ e $k_{1}$}

A partir dos estimadores de máxima verossimilhança $\hat{\lambda} \mathrm{e} \hat{\mathrm{k}}_{1}$, podemos obter intervalos de confiança e testes de hipóteses para os parâmetros do modelo, usando a teoria assintótica para grandes amostras, baseadas na matriz de informação de Fisher ou na matriz de informação observada. Assim, consideramos o resultado,

$$
\left(\hat{\lambda}, \widehat{\mathrm{k}}_{1}\right) \stackrel{\mathrm{a}}{\sim} N\left[\left(\lambda, \mathrm{k}_{1}\right), \mathrm{I}^{-1}\left(\hat{\lambda}, \widehat{\mathrm{k}}_{1}\right)\right]
$$


A matriz de informação observada é definida por:

$$
\mathrm{I}\left(\hat{\lambda}, \hat{\mathrm{k}}_{1}\right)=\left[\begin{array}{cc}
-\frac{\partial^{2} 1\left(\hat{\lambda}, \hat{\mathrm{k}}_{1}\right)}{\partial^{2} \lambda} & -\frac{\partial^{2} 1\left(\hat{\lambda}, \hat{\mathrm{k}}_{1}\right)}{\partial \lambda \partial \mathrm{k}_{1}} \\
-\frac{\partial^{2} 1\left(\hat{\lambda}, \hat{\mathrm{k}}_{1}\right)}{\partial \lambda \partial \mathrm{k}_{1}} & -\frac{\partial^{2} 1\left(\hat{\lambda}, \hat{\mathrm{k}}_{1}\right)}{\partial^{2} \mathrm{k}_{1}}
\end{array}\right]
$$

onde,

$$
\begin{aligned}
& I_{11}=-\frac{\partial^{2} l\left(\hat{\lambda}, \hat{k}_{1}\right)}{\partial^{2} \lambda}=\frac{\sum_{i=1}^{n} m_{i}}{\hat{\lambda}^{2}}, \\
& I_{22}=-\frac{\partial^{2} l\left(\hat{\lambda}, \hat{k}_{1}\right)}{\partial^{2} k_{1}}=\hat{\lambda} \sum_{i=1}^{n} \frac{\hat{k}_{1}^{i-2}(i-1)(i-2)}{\hat{k}_{1}}+\sum_{i=1}^{n} \frac{m_{i}(i-1)}{\hat{k}_{1}^{2}}, \\
& I_{21}=I_{12}=-\frac{\partial^{2} 1\left(\hat{\lambda}, \hat{k}_{1}\right)}{\partial \lambda \partial \mathrm{k}_{1}}=\frac{\sum_{i=1}^{n} \hat{k}_{1}^{i-1}(i-1)}{\hat{k}_{1}} .
\end{aligned}
$$

e $I^{-1}\left(\hat{\lambda}, \hat{k}_{1}\right)$, o inverso da matriz de informação observada, dada em (2.1.7), representa a matriz de variância e covariância assintótica de $\hat{\lambda}_{\mathrm{e}} \hat{\mathrm{k}}_{1}$.

A partir da normalidade assintótica dos estimadores, podemos construir intervalos de confiança e testes de hipóteses para $\lambda$ e $\mathrm{k}_{1}$, usando a inversa da matriz de informação observada. 
Considerando-se, por exemplo, um nível de significância $(1-\alpha)$, os intervalos de confiança assintóticos para $\lambda$ e $\mathrm{k}_{1}$ são dados por:

$$
\operatorname{Ic}(\lambda)=\left[\hat{\lambda}-z_{\alpha / 2} \sqrt{\mathrm{I}_{11}^{-1}\left(\hat{\lambda}, \widehat{\mathrm{k}}_{1}\right)} ; \hat{\lambda}+z_{\alpha / 2} \sqrt{\mathrm{I}_{11}^{-1}\left(\hat{\lambda}, \widehat{\mathrm{k}}_{1}\right)}\right]
$$

e

$$
\operatorname{Ic}\left(\mathrm{k}_{1}\right)=\left[\hat{\mathrm{k}}_{1}-\mathrm{z}_{\alpha / 2} \sqrt{\mathrm{I}_{22}^{-1}\left(\hat{\lambda}, \widehat{\mathrm{k}}_{1}\right)} ; \hat{\mathrm{k}}_{1}+\mathrm{z}_{\alpha / 2} \sqrt{\mathrm{I}_{22}^{-1}\left(\hat{\lambda}, \widehat{\mathrm{k}}_{1}\right)}\right]
$$

onde $\mathrm{I}_{\mathrm{ii}}^{-1}\left(\hat{\lambda}, \hat{\mathrm{k}}_{1}\right)$ representa o i-ésimo elemento diagonal da matriz de variância e covariância assintótica de $\hat{\lambda}$ e $\hat{k}_{1}$, e $z_{\alpha / 2}$ é um percentil da distribuição normal padronizada de uma tabela estatística.

\subsection{Análise Clássica da Generalização do Modelo de Moranda (Achcar, 1995)}

Nesta seção, discutimos algumas motivações para a generalização do modelo de Moranda. Inicialmente, determinamos os estimadores de máxima verossimilhança desse modelo e, a partir da normalidade assintótica desses estimadores, obtemos as inferências sobre os parâmetros de interesse.

\subsubsection{Motivação para a Generalização do Modelo de Moranda (1975) (Achcar, 1995)}

Em confiabilidade de software, quando assumimos um modelo para modelar os dados de falhas ${ }^{1}$, um dos interesses mais importantes é o comportamento da taxa de falha do modelo.

Dados de falhas podem ser classificados em tempos entre falhas e número de falhas observadas no intervalo de falha $(0, \mathrm{t}]$. 
Observe-se que um modelo de confiabilidade com taxa de falha, apresentando grande flexibidade, proporciona um bom ajuste do modelo aos dados e permite que o modelo seja considerado para uma extensa classe de distribuições para os dados de falhas.

Entretanto, o modelo de Moranda apresenta taxa de falha somente decrescente; dessa forma, esse modelo é restrito apenas à classe de distribuições para os dados de falhas com taxa de falha decrescente.

Para melhorar a flexibilidade da taxa de falha desse modelo, Achcar (1995) propõe uma modificação. Essa generalização do modelo em estudo pode ser dada adicionando-se um parâmetro $\mathrm{k}_{2}$ na função intensidade (2.1.1), e uma reparametrização $\lambda_{\mathrm{a}}=\frac{\lambda}{\mathrm{k}_{1}}$, isto é,

$$
\lambda_{\mathrm{i}}=\lambda_{\mathrm{a}} \mathrm{k}_{1}^{\mathrm{i}_{2}} \text {, onde } \lambda_{\mathrm{a}}>0,0<\mathrm{k}_{1}<1 \text { e }-\infty<\mathrm{k}_{2}<\infty
$$

A reparametrização $\lambda_{\mathrm{a}}=\frac{\lambda}{\mathrm{k}_{1}}$ é feita somente com objetivo de facilitar os cálculos computacionais.

Observe que se considerarmos $\mathrm{k}_{2}=1$ e $\lambda_{\mathrm{a}}=\frac{\lambda}{\mathrm{k}_{1}}$, temos a taxa de falha (2.1.1) do modelo de Moranda.

$\mathrm{O}$ parâmetro $\mathrm{k}_{2}$, presente na generalização do modelo de Moranda, permite maior flexibilidade de ajuste se comparado ao modelo de Moranda com 2 parâmetros (ver figura 2.2), e sua interpretação é inerente ao comportamento de falhas que se deseja analisar. 


$$
\lambda_{\mathrm{i}}=\lambda_{\mathrm{a}} \mathrm{k}_{1}^{\mathrm{i}_{2}}
$$

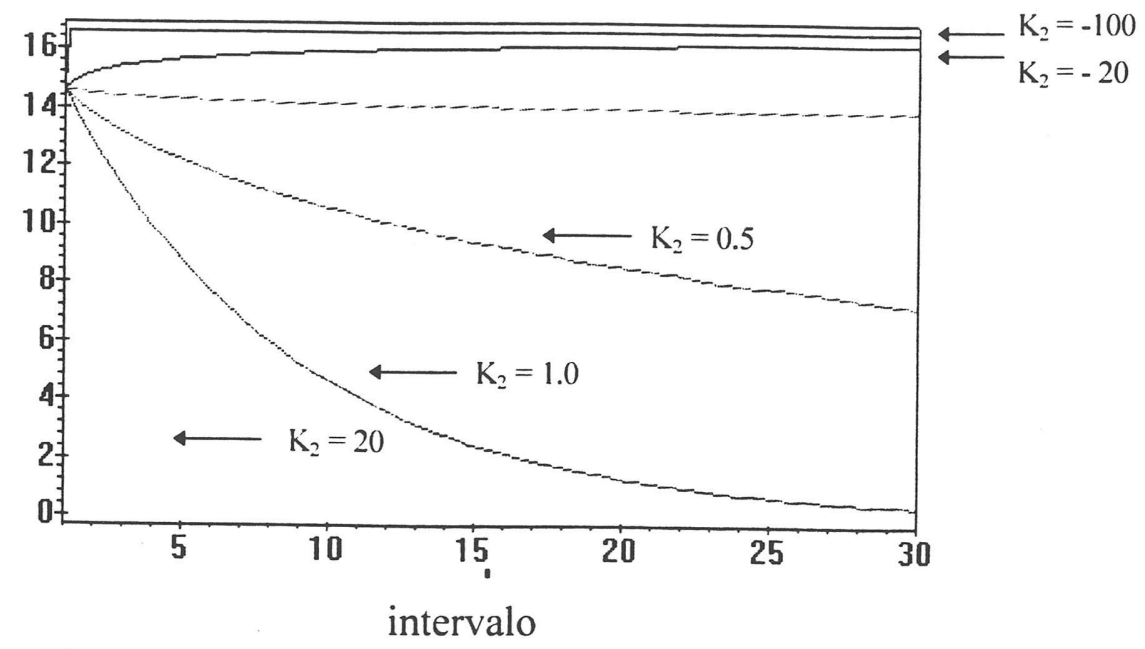

Figura 2.2: Taxa de falha da generalização do Modelo de Moranda (Achcar, 1995) com $\lambda_{\mathrm{a}}=16 \mathrm{e} \mathrm{k}_{1}=0.88$, para seis valores de $k_{2}$.

Quando inserimos o parâmetro $\mathrm{k}_{2}$ na função intensidade (2.1.1), a taxa de falha é crescente, decrescente e constante, semelhante à distribuição de Weibull, que têm sido extensivamente utilizada em estudos de confiabilidade, devido a essa propriedade da taxa de falha (crescente, decrescente e constante).

No Capítulo 6, podemos verificar, com alguns conjuntos de dados, a melhoria significativa que a forma generalizada do modelo de Moranda proporciona ao ajuste do modelo.

\subsubsection{Função de Verossimilhança}

A função de verossimilhança para a generalização do modelo de Moranda (Achcar, 1995), $\mathrm{L}\left(\lambda_{\mathrm{a}}, \mathrm{k}_{1}, \mathrm{k}_{2}\right)$, considerando-se uma amostra de tamanho $n$, é escrita na forma: 


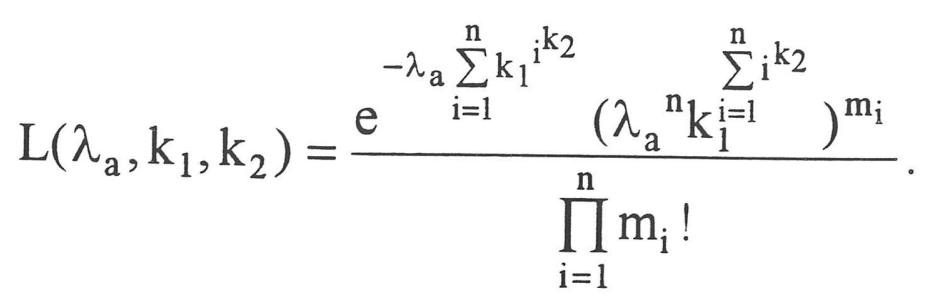

\subsubsection{Estimadores de Máxima Verossimilhança}

Os estimadores de máxima verossimilhança são os valores de $\lambda_{\mathrm{a}}, \mathrm{k}_{1}$ e $\mathrm{k}_{2}$, que maximizam $\mathrm{L}\left(\lambda_{\mathrm{a}}, \mathrm{k}_{1}, \mathrm{k}_{2}\right)$, ou a função log-verossimilhança.

Para a forma generalizada do modelo de Moranda, a função logverossimilhança, ignorando o termo que não depende dos parâmetros, fica especificada por:

$$
\mathrm{l}\left(\lambda_{\mathrm{a}}, \mathrm{k}_{1}, \mathrm{k}_{2}\right) \propto \sum_{\mathrm{i}=1}^{\mathrm{n}} \mathrm{m}_{\mathrm{i}} \log \lambda_{\mathrm{a}}+\sum_{\mathrm{i}=1}^{\mathrm{n}} \mathrm{i}^{\mathrm{k}_{2}} \mathrm{~m}_{\mathrm{i}} \log \mathrm{k}_{1}-\lambda_{\mathrm{a}} \sum_{\mathrm{i}=1}^{\mathrm{n}} \mathrm{k}_{1} \mathrm{i}^{\mathrm{k}_{2}}
$$

Calculando as primeiras derivadas parciais, em relação aos parâmetros $\lambda_{\mathrm{a}}, \mathrm{k}_{1}$ e $\mathrm{k}_{2}$, temos:

$$
\begin{aligned}
& \frac{\partial \mathrm{l}\left(\lambda_{\mathrm{a}}, \mathrm{k}_{1}, \mathrm{k}_{2}\right)}{\partial \lambda_{\mathrm{a}}}=-\frac{\sum_{\mathrm{i}=1}^{\mathrm{n}} \mathrm{m}_{\mathrm{i}}}{\lambda_{\mathrm{a}}}+\sum_{\mathrm{i}=1}^{\mathrm{n}} \mathrm{k}_{1}^{\mathrm{i}_{2}}, \\
& \frac{\partial \mathrm{l}\left(\lambda_{\mathrm{a}}, \mathrm{k}_{1}, \mathrm{k}_{2}\right)}{\partial \mathrm{k}_{1}}=-\lambda_{\mathrm{a}} \sum_{\mathrm{i}=1}^{\mathrm{n}} \frac{\mathrm{k}_{1}^{\mathrm{i}^{\mathrm{k}} 2} \mathrm{i}^{\mathrm{k}_{2}}}{\mathrm{k}_{1}}+\sum_{\mathrm{i}=1}^{\mathrm{n}} \frac{\mathrm{i}^{\mathrm{k}_{2}} \mathrm{~m}_{\mathrm{i}}}{\mathrm{k}_{1}}
\end{aligned}
$$




$$
\begin{aligned}
& \frac{\partial \mathrm{l}\left(\lambda_{\mathrm{a}}, \mathrm{k}_{1}, \mathrm{k}_{2}\right)}{\partial \mathrm{k}_{2}}=-\lambda_{\mathrm{a}}\left(\sum_{\mathrm{i}=1}^{\mathrm{n}} \mathrm{k}_{1}^{\mathrm{i}^{\mathrm{k}_{2}}} \mathrm{i}^{\mathrm{k}_{2}} \log (\mathrm{i}) \log \left(\mathrm{k}_{1}\right)\right)+ \\
& \left(\sum_{\mathrm{i}=1}^{\mathrm{n}} \mathrm{i}^{\mathrm{k}_{2}} \log (\mathrm{i}) \mathrm{m}_{\mathrm{i}}\right) \log \left(\mathrm{k}_{1}\right) .
\end{aligned}
$$

Os estimadores de máxima verossimilhança $\hat{\mathrm{k}}_{1}$ e $\hat{\mathrm{k}}_{2}$ não podem ser obtidos de modo analítico, portanto, devemos maximizar (2.2.3), utilizando um método iterativo, como, por exemplo, o método de Newton.

O estimador de máxima verossimilhança de $\lambda_{\mathrm{a}}$ é dado por:

$$
\hat{\lambda}_{a}=\frac{\sum_{i=1}^{n} m_{i}}{\sum_{i=1}^{n} \hat{k}_{1}^{\hat{i}_{2}}} .
$$

\subsubsection{Inferências Sobre os Parâmetros $\lambda_{\mathrm{a}}, \mathrm{k}_{1}$ e $\mathrm{k}_{2}$}

Pela normalidade assintótica dos estimadores de máxima verossimilhança temos:

$$
\left(\bar{\lambda}_{\mathrm{a}}, \widehat{\mathrm{k}}_{1}, \widehat{\mathrm{k}}_{2}\right) \stackrel{\mathrm{a}}{\sim} \mathbf{N}\left[\left(\lambda_{\mathrm{a}}, \mathrm{k}_{1}, \mathrm{k}_{2}\right), \mathbf{I}^{-1}\left(\hat{\lambda}_{\mathrm{a}}, \widehat{\mathrm{k}}_{1}, \widehat{\mathrm{k}}_{2}\right)\right]
$$

Assim, para a forma generalizada do modelo de Moranda, a matriz de informação observada é dada por: 


$$
\mathrm{I}\left(\hat{\lambda}_{\mathrm{a}}, \hat{\mathrm{k}}_{1}, \hat{\mathrm{k}}_{2}\right)=\left[\begin{array}{ccc}
-\frac{\partial \mathrm{l}^{2}\left(\hat{\lambda}_{\mathrm{a}}, \hat{\mathrm{k}}_{1}, \hat{\mathrm{k}}_{2}\right)}{\partial^{2} \lambda_{\mathrm{a}}} & -\frac{\partial \mathrm{l}\left(\hat{\lambda}_{\mathrm{a}}, \hat{\mathrm{k}}_{1}, \hat{\mathrm{k}}_{2}\right)}{\partial \lambda_{\mathrm{a}} \mathrm{k}_{1}} & -\frac{\partial \mathrm{l}\left(\hat{\lambda}_{\mathrm{a}}, \hat{\mathrm{k}}_{1}, \hat{\mathrm{k}}_{2}\right)}{\partial \lambda_{\mathrm{a}} \mathrm{k}_{2}} \\
-\frac{\partial \mathrm{l}\left(\hat{\lambda}_{\mathrm{a}}, \hat{\mathrm{k}}_{1}, \hat{\mathrm{k}}_{2}\right)}{\partial \lambda_{\mathrm{a}} \mathrm{k}_{1}} & -\frac{\partial \mathrm{l}^{2}\left(\hat{\lambda}_{\mathrm{a}}, \hat{\mathrm{k}}_{1}, \hat{\mathrm{k}}_{2}\right)}{\partial^{2} \mathrm{k}_{1}} & -\frac{\partial \mathrm{l}\left(\hat{\lambda}_{\mathrm{a}}, \hat{\mathrm{k}}_{1}, \hat{\mathrm{k}}_{2}\right)}{\partial \mathrm{k}_{1} \partial \mathrm{k}_{2}} \\
-\frac{\partial \mathrm{l}\left(\hat{\lambda}_{\mathrm{a}}, \hat{\mathrm{k}}_{1}, \hat{\mathrm{k}}_{2}\right)}{\partial \lambda_{\mathrm{a}} \mathrm{k}_{2}} & -\frac{\partial \mathrm{l}\left(\hat{\lambda}_{\mathrm{a}}, \hat{\mathrm{k}}_{1}, \hat{\mathrm{k}}_{2}\right)}{\partial \mathrm{k}_{1} \partial \mathrm{k}_{2}} & -\frac{\partial \mathrm{l}^{2}\left(\hat{\lambda}_{\mathrm{a}}, \hat{\mathrm{k}}_{1}, \hat{\mathrm{k}}_{2}\right)}{\partial^{2} \mathrm{k}_{2}}
\end{array}\right]
$$

onde,

$$
\begin{aligned}
& I_{11}=-\frac{\partial 1^{2}\left(\hat{\lambda}_{a}, \hat{k}_{1}, \hat{k}_{2}\right)}{\partial^{2} \lambda_{a}}=\frac{\sum_{i=1}^{n} m_{i}}{\hat{\lambda}_{a}^{2}}, \\
& \mathrm{I}_{22}=-\frac{\partial \mathrm{l}^{2}\left(\hat{\lambda}_{\mathrm{a}}, \hat{\mathrm{k}}_{1}, \hat{\mathrm{k}}_{2}\right)}{\partial^{2} \mathrm{k}_{1}}=\bar{\lambda}_{\mathrm{a}} \sum_{\mathrm{i}=1}^{\mathrm{n}} \overline{\mathrm{k}}_{1}^{\mathrm{i}^{\mathrm{k}_{2}}-2} \mathrm{i}^{\mathrm{k}_{2}}\left(\mathrm{i}^{\mathrm{k}_{2}}-1\right)+\frac{\sum_{\mathrm{i}=1}^{\mathrm{n}} \mathrm{i}^{\hat{\mathrm{k}}_{2}} \mathrm{~m}_{\mathrm{i}}}{\widehat{\mathrm{k}}_{1}^{2}}, \\
& I_{33}=-\frac{\partial \mathrm{l}^{2}\left(\hat{\lambda}_{\mathrm{a}}, \hat{\mathrm{k}}_{1}, \hat{\mathrm{k}}_{2}\right)}{\partial^{2} \mathrm{k}_{2}}=\bar{\lambda}_{\mathrm{a}} \sum_{\mathrm{i}=1}^{\mathrm{n}}\left(\widehat{\mathrm{k}}_{1}^{\mathrm{i}^{\mathrm{k}_{2}}}\left(\mathrm{i}^{\mathrm{k}_{2}}\right)^{2}(\log \mathrm{i})^{2}\left(\log \widehat{\mathrm{k}}_{1}\right)^{2}\right. \\
& \left.-\widehat{\mathrm{k}}_{1}^{\mathrm{k}^{\mathrm{k}}}(\log (\mathrm{i}))^{2} \mathrm{i}^{\mathrm{k}_{2}} \log \left(\widehat{\mathrm{k}}_{1}\right)\right)+\sum_{\mathrm{i}=1}^{\mathrm{n}}\left(\mathrm{i}^{\mathrm{k}_{2}}\left(\log \left(\widehat{\mathrm{k}}_{1}\right)\right) \log (\mathrm{i})^{2} \mathrm{mi}\right),
\end{aligned}
$$

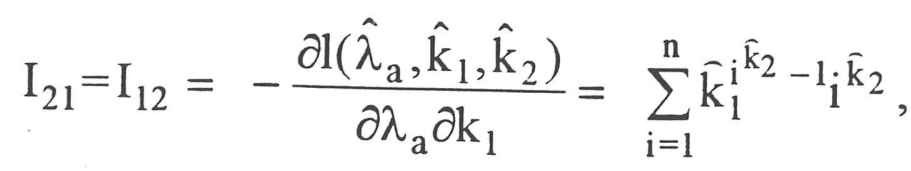

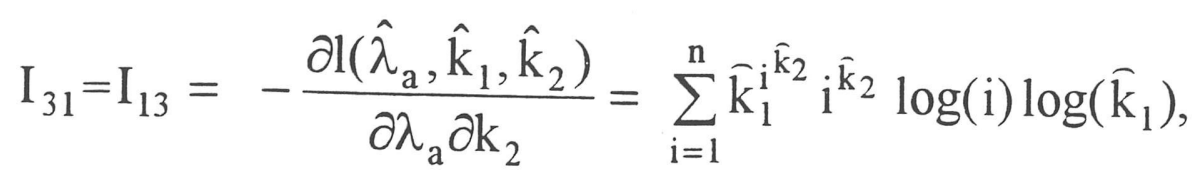




$$
\begin{aligned}
& \mathrm{I}_{32}=\mathrm{I}_{23}=-\frac{\partial \mathrm{l}\left(\hat{\lambda}_{\mathrm{a}}, \hat{\mathrm{k}}_{1}, \hat{\mathrm{k}}_{2}\right)}{\partial \mathrm{k}_{1} \partial \mathrm{k}_{2}}=\hat{\lambda}_{\mathrm{a}} \sum_{\mathrm{i}=1}^{\mathrm{n}} \widehat{\mathrm{k}}_{1}^{\mathrm{i}^{\mathrm{k}}{ }^{2}-1}\left(\mathrm{i}^{\hat{\mathrm{k}}_{2}}\right)^{2} \log (\mathrm{i}) \log \left(\hat{\mathrm{k}}_{1}\right) \\
& +\sum_{\mathrm{i}=1}^{\mathrm{n}} \widehat{\mathrm{k}}_{1}^{\mathrm{i}^{\hat{\mathrm{k}}_{2}}-1}\left(\mathrm{i}^{\mathrm{k}_{2}}\right) \log (\mathrm{i})-\sum_{\mathrm{i}=1}^{\mathrm{n}} \frac{\mathrm{i}^{\hat{\mathrm{k}}_{2}} \log (\mathrm{i}) \mathrm{m}_{\mathrm{i}}}{\widehat{\mathrm{k}}_{1}}
\end{aligned}
$$

Portanto, considerando um nível de significância $100(1-\alpha) \%$, temos os intervalos de confiança para $\lambda_{\mathrm{a}}, \mathrm{k}_{1}$ e $\mathrm{k}_{2}$ dados por:

$$
\begin{aligned}
& \operatorname{Ic}\left(\lambda_{\mathrm{a}}\right)=\left[\hat{\lambda}-\mathrm{z}_{\alpha / 2} \mathrm{I}_{11}^{-1 / 2}\left(\hat{\lambda}_{\mathrm{a}}, \widehat{\mathrm{k}}_{1,}, \widehat{\mathrm{k}}_{2}\right) ; \hat{\lambda}+\mathrm{z}_{\alpha / 2} \mathrm{I}_{11}^{-1 / 2}\left(\hat{\lambda}_{\mathrm{a}}, \widehat{\mathrm{k}}_{1}, \widehat{\mathrm{k}}_{2}\right)\right] \\
& \operatorname{Ic}\left(\mathrm{k}_{1}\right)=\left[\hat{\mathrm{k}}_{1}-\mathrm{z}_{\alpha / 2} \mathrm{I}_{22}^{-1 / 2}\left(\hat{\lambda}_{\mathrm{a}}, \widehat{\mathrm{k}}_{1}, \widehat{\mathrm{k}}_{2}\right) ; \hat{\mathrm{k}}_{1}+\mathrm{z}_{\alpha / 2} \mathrm{I}_{22}^{-1 / 2}\left(\hat{\lambda}_{\mathrm{a}}, \widehat{\mathrm{k}}_{1}, \widehat{\mathrm{k}}_{2}\right)\right] \\
& \operatorname{Ic}\left(\mathrm{k}_{2}\right)=\left[\hat{\mathrm{k}}_{2}-\mathrm{z}_{\alpha / 2} \mathrm{I}_{33}^{-1 / 2}\left(\hat{\lambda}_{\mathrm{a}}, \widehat{\mathrm{k}}_{1}, \widehat{\mathrm{k}}_{2}\right) ; \hat{\mathrm{k}}_{2}+\mathrm{z}_{\alpha / 2} \mathrm{I}_{33}^{-1 / 2}\left(\hat{\lambda}_{\mathrm{a}}, \widehat{\mathrm{k}}_{1}, \widehat{\mathrm{k}}_{2}\right)\right]
\end{aligned}
$$

onde $\mathrm{I}_{\mathrm{ii}}^{-1}\left(\widehat{\lambda}_{\mathrm{a}}, \widehat{\mathrm{k}}_{1}, \widehat{\mathrm{k}}_{2}\right)$ é o i-ésimo elemento diagonal da matriz de variância e covariâncias de $\lambda_{\mathrm{a}}, \mathrm{k}_{1} \mathrm{e} \mathrm{k}_{2}$, ou seja, o inverso da matriz de informação observada, definida em (2.2.4), e $z_{\alpha / 2}$ é um percentil da distribuição normal padronizada.

A qualidade de predição da confiabilidade e a de outras medidas associadas ao comportamento das falhas do software (por exemplo, taxa de falha, previsões, etc) são muito importantes, e várias decisões de engenharia dependem disso, como, por exemplo, a liberação do software no mercado. Uma estimativa errada, "viciada", da confiabilidade ou da taxa de falha do software acarreta decisões equivocadas em relação ao mesmo, daí a importância de resultados precisos. 
Devemos ressaltar, no entanto, que a obtenção de resultados precisos para os parâmetros dos modelos de confiabilidade de software pelo método clássico usual depende do tamanho da amostra ou de uma reparametrização adequada. Dependendo do modelo e conjuntos de dados, podemos ter dificuldades na obtenção de inferências, ou resultados imprecisos para os parâmetros de interesse. 


\section{Capítulo 3}

\section{ANÁLISE BAYESIANA PARA ALGUNS MODELOS DE ESTRATÉGIA TIPO II}

Em análise de confiabilidade de software, quando assumimos um modelo para o número de falhas do software $\mathrm{M}(\mathrm{t})$, observadas durante um intervalo de tempo $[0, t)$, temos interesse em determinar o valor do vetor $\beta=\left\{\beta_{1}, \beta_{2}, \ldots, \beta_{n}\right\}$ de parâmetros que o definem, ou de funções dos mesmos.

Usualmente, os pesquisadores de software têm opinião prévia sobre $\beta$ e, em inferência Bayesiana, essa opinião pode ser incorporada à análise através de distribuições de probabilidade a priori para os parâmetros de interesse que, sob esse ponto de vista, tais parâmetros são considerados variáveis aleatórias.

A fórmula de Bayes (ver por exemplo Box e Tiao, 1973) proporciona um método para calcular as densidades de probabilidades dos parâmetros de interesse, combinando a informação contida nos dados com a informação prévia que o pesquisador tem a respeito de $\beta$.

Para representar a opinião prévia sobre os parâmetros, vamos considerar densidades a priori informativas para os parâmetros do modelo de Moranda (1975) e da forma generalizada desse modelo (Achcar, 1995), para, assim, obter as densidades a posteriori de interesse. Vamos explorar o uso de algoritmos computacionais, como o amostrador de Gibbs, para implementar os cálculos Bayesianos. Quando as densidades condicionais no algoritmo de Gibbs não são facilmente identificáveis, utilizamos o algoritmo de Gibbs e Metropolis-Hastings (ver Gelfand e Smith, 1990). 


\subsection{Fórmula de Bayes}

Sejam $X$ e $\beta_{1}$ variáveis aleatórias com densidades conjuntas e condicionais dadas por $\pi\left(x, \beta_{1}\right), \pi\left(x \backslash \beta_{1}\right)$, e $\pi\left(\beta_{1} \backslash x\right)$, respectivamente; e seja $\pi\left(\beta_{1}\right)$ a densidade marginal de $\beta_{1}$. A densidade condicional de $\beta_{1}$, dado $\mathrm{x}$, é dada por,

$$
\pi\left(\beta_{1} \backslash \mathrm{x}\right)=\frac{\pi\left(\mathrm{x}, \beta_{1}\right)}{\pi(\mathrm{x})}
$$

Isto é,

$$
\pi\left(\beta_{1} \backslash x\right)=\frac{\pi\left(x \backslash \beta_{1}\right) \pi\left(\beta_{1}\right)}{\int_{\beta_{1} \in \Theta} \pi\left(x \backslash \beta_{1}\right) \pi\left(\beta_{1}\right) d \beta_{1}},
$$

onde $\Theta$ é o espaço paramétrico.

\subsection{Densidades a Priori e a Posteriori}

A utilização de métodos Bayesianos na análise estatística dos dados permite a incorporação de conhecimento prévio sobre $\beta$, através de uma densidade de probabilidade para $\beta$. Essa densidade é identificada como densidade a priori e é denotada por $\pi(\beta)$. Quando não temos nenhuma informação a respeito de $\beta$, devemos formular uma informação a priori, tal que toda a informação acerca do parâmetro provenha exclusivamente dos dados, ou seja, devemos considerar uma densidade a priori não-informativa.

Uma vez definidos o modelo para os dados e a distribuição a priori para $\beta$, devemos combinar a informação prévia sobre o parâmetro $\beta$ à informação contida nos dados, obtendo assim uma distribuição a posteriori para $\beta$. 
Se $\pi(\beta)$ representa a distribuição a priori do vetor de parâmetros desconhecidos e $\mathrm{p}(\beta \backslash \mathrm{x})$ é a função de verossimilhança, frequentemente denotada por $\mathrm{L}(\beta)$, podemos obter as distribuições a posteriori conjuntas e marginais para os parâmetros $\beta=\left\{\beta_{1}, \beta_{2}, \ldots, \beta_{\mathrm{n}}\right\}$.

Utilizando a expressão (3.1.1) temos que a distribuição a posteriori para o vetor de parâmetros $\beta$ é dada por:

$$
\pi(\beta \backslash \mathrm{x})=\frac{\mathrm{L}(\beta) \pi(\beta)}{\int_{\beta \in \Theta} \mathrm{L}(\beta) \pi(\beta) \mathrm{d} \beta},
$$

onde $\Theta$ é o espaço paramétrico.

A distribuição a posteriori (3.2.1) pode ser utilizada para a estimativa pontual do parâmetro de interesse (por exemplo, a média ou a moda a posteriori de $\beta$ ) e para a construção de intervalos de credibilidade (estimativa Bayesiana por intervalo).

Em muitas situações, para encontrarmos as distribuições a posteriori marginais, densidades preditivas, ou momentos a posteriori de interesse, não conseguimos achar uma forma analítica explícita para essas integrais. Nesse caso, escolhe-se uma entre as várias estratégias existentes tais como: métodos numéricos (Naylor e Smith, 1982); métodos de aproximação de integrais (Tierney e Kadane, 1986); métodos de Monte Carlo (Kloek e Van Dijk, 1978) ou algoritmos de simulação de Monte Carlo com Cadeia de Markov (MCMC), do tipo MetropolisHastings (Chib e Greenberg, 1995) e amostrador de Gibbs (Gelfand e Smith, 1990; Casella e George, 1992).

Neste trabalho, exploramos os algoritmos computacionais MCMC, pois os modelos de confiabilidade de software, principalmente os que utilizam processos de Poisson não homogêneo, apresentam vários parâmetros. Desta forma, as integrais Bayesianas de interesse são integrais multidimensionais, tornando-se necessária a 
utilização de métodos computacionais para a solução das mesmas.

Utilizamos os algoritmos amostrador de Gibbs e Metropolis-Hastings porque tais amostradores são técnicas que permitem a geração de variáveis aleatórias de uma distribuição (marginal) indiretamente, isto é, sem necessidade do cálculo da densidade marginal, o que implementa o cálculo das integrais com um procedimento muito simples, comparado aos demais métodos de aproximação de integrais.

\subsection{Algoritmos de Simulação de Monte Carlo com Cadeia de Markov}

Um dos tópicos mais importantes na estatística computacional é a inferência realizada através de simulação iterativa, especialmente feita pelo algoritmo conhecido como amostrador de Gibbs e o algoritmo de Metropolis-Hastings (ver por exemplo, Gelfand e Smith, 1990; Casella e George, 1992; Chib e Greenberg, 1995). Esta metodologia tem causado grande impacto em problemas paramétricos principalmente no uso de modelos Bayesianos.

$O$ amostrador de Gibbs é uma técnica que envolve a seleção de amostras aleatórias usando a densidade conjunta $\mathrm{p}(\beta \backslash$ dados), onde $\beta$ é multiparamétrico, quando as distribuições condicionais são de forma padronizada como Normal, Gama, Poisson, Beta, etc.

Quando essas distribuições condicionais não são facilmente identificáveis, usamos um método mais geral que tem o amostrador de Gibbs como um caso particular, o algoritmo de Metropolis-Hastings (ver por exemplo Gelfand e Smith, 1990; Chib e Greenberg, 1995). Os algoritmos amostrador de Gibbs e MetropolisHastings são apresentados no apêndice A.

\subsubsection{Convergência dos Algoritmos Amostrador de Gibbs e Metropolis-Hastings}

A convergência (em distribuição) dos algoritmos amostrador de Gibbs e Metropolis-Hastings pode ser verificada de várias formas. Gerando-se, por exemplo, m seqüências de comprimento $\mathrm{M}+\mathrm{N}$, cada uma partindo de uma condição inicial diferente e escolhendo-se, a seguir as m seqüências de tamanho $\mathrm{N}$, temos 
uma amostra i.i.d dos parâmetros que estão sendo gerados. Geman e Geman (1984) argumentam que essa amostra converge em distribuição para uma amostra i.i.d da densidade marginal procurada.

Um critério de verificação formal de convergência que utilizamos nesse trabalho é o proposto por Gelman e Rubin (1992). Esse critério utiliza a técnica de análise de variância para verificar a convergência em distribuição do algoritmo amostrador de Gibbs, calculando a variabilidade entre as cadeias e dentro das cadeias de amostras geradas por esse amostrador.

Para verificar a convergência em distribuição, utiliza-se o fator de redução de escala denotado em Gelman e Rubin (1992) por $\sqrt{\hat{\mathrm{R}}}$. Se esse fator é maior que 1 , devemos considerar mais simulações, para melhorar a inferência sobre a distribuição de interesse; se $\sqrt{\hat{R}}$ estiver próximo de 1 , a convergência foi atingida e as amostras selecionadas das iterações são i.i.d. O critério de Gelman e Rubin é apresentado no apêndice A.3.

\subsection{Análise Bayesiana Para o Modelo de Moranda Considerando Densidades a Priori Informativas}

Nesta seção, apresentamos uma análise Bayesiana do modelo de Moranda, considerando densidades a priori informativas para representar o grau de conhecimento sobre os parâmetros $\lambda$ e $\mathrm{k}_{1}$, e utilizamos algoritmos computacionais como o amostrador de Gibbs e Metropolis-Hastings (ver Gelfand e Smith, 1990) para aproximar as densidades a posteriori de interesse.

\subsubsection{Densidades $\boldsymbol{a}$ Priori Informativas para $\lambda$ e $\mathrm{k}_{1}$}

Como comentamos na seção (3.2), o conhecimento prévio do pesquisador, a respeito dos parâmetros é expresso pela densidade a priori.

Assumindo independência entre os parâmetros do modelo (2.1.1), uma densidade a priori conjunta para $\lambda$ e $\mathrm{k}_{1}$ é dada por: 


$$
\pi\left(\lambda, \mathrm{k}_{1}\right) \propto \pi(\lambda) \pi\left(\mathrm{k}_{1}\right)
$$

Para expressar o grau de conhecimento sobre os parâmetros do modelo de Moranda, assumimos $\mathrm{k}_{1}$, com distribuição a priori Beta $\left(\mathrm{c}_{1}, \mathrm{c}_{2}\right)\left(\right.$ para $\left.0<\mathrm{k}_{1}<1\right)$ e $\lambda$, com distribuição a priori Gama $\left(b_{1}, b_{2}\right)$ (para $\lambda>0$ ). Desta forma, (3.4.1) é dada por,

$$
\pi\left(\lambda, \mathrm{k}_{1}\right) \propto \lambda^{\mathrm{b}_{1}-1} \mathrm{e}^{-\mathrm{b}_{2} \lambda_{\mathrm{a}}} \mathrm{k}_{1}^{\mathrm{c}_{1}-1}\left(1-\mathrm{k}_{1}\right)^{\mathrm{c}_{2}-1}
$$

onde $\lambda>0$ e $0<\mathrm{k}_{1}<1$.

$\mathrm{Na}$ prática, os valores $\mathrm{c}_{1}, \mathrm{c}_{2}, \mathrm{~b}_{1}$ e $\mathrm{b}_{2}$ da expressão (3.4.2) podem ser determinados a partir de informações como a média e a variabilidade do parâmetro de interesse, colhidas junto ao especialista da área de software, ou através de estudos preliminares dos dados de falhas.

\subsubsection{Densidade a Posteriori Conjunta para $\lambda$ e $\mathrm{k}_{1}$}

Combinando a densidade a priori (3.4.2) com a função de verossimilhança (2.1.3), obtemos a densidade a posteriori conjunta, dada pela expressão:

$$
\begin{aligned}
& \pi\left(\lambda, \mathrm{k}_{1} \backslash \mathrm{m}_{1}, \mathrm{~m}_{2}, \ldots, \mathrm{m}_{\mathrm{n}}\right) \propto \lambda^{\sum_{\mathrm{i}=1}^{\mathrm{n}} \mathrm{m}_{\mathrm{i}}+\mathrm{b}_{1}-1} \\
& \exp -\left(\left[\mathrm{b}_{2}+\sum_{\mathrm{i}=1}^{\mathrm{n}} \mathrm{k}_{1}^{\mathrm{i}-1}\right] \lambda\right) \mathrm{k}_{1}^{\mathrm{i}=1} \mathrm{i}^{\mathrm{i}-1) \mathrm{m}_{\mathrm{i}}+\mathrm{c}_{1}-1}\left(1-\mathrm{k}_{1}\right)^{\mathrm{c}_{2}-1}
\end{aligned}
$$

onde $\lambda>0$ e $0<\mathrm{k}_{1}<1$. 


\subsubsection{Densidade Condicional de $\lambda$ para o Algoritmo amostrador de Gibbs}

A densidade condicional de $\lambda$ para o algoritmo amostrador de Gibbs é obtida através da densidade a posteriori conjunta de $\lambda$ e $\mathrm{k}_{1}$ (3.4.3), considerando $\lambda$ condicionado aos dados e ao parâmetro $\mathrm{k}_{1}$. Assim, temos,

$$
\pi\left(\lambda / \mathrm{k}_{1}, \mathrm{~m}_{1}, \mathrm{~m}_{2}, \ldots, \mathrm{m}_{\mathrm{n}}\right) \sim \Gamma\left(\sum_{\mathrm{i}=1}^{\mathrm{n}} \mathrm{m}_{\mathrm{i}}+\mathrm{b}_{1}, \mathrm{~b}_{2}+\sum_{\mathrm{i}=1}^{\mathrm{n}} \mathrm{k}_{1}^{\mathrm{i}-1}\right)
$$

onde $\lambda>0$ e $0<\mathrm{k}_{1}<1$.

A densidade de $\lambda$, condicionada aos dados e ao parâmetro $\mathrm{k}_{1}$ (3.4.4), tem forma padronizada gama. Como comentamos na seção (3.3), quando identificamos uma distribuição condicional de forma conhecida, utilizamos 0 algoritmo amostrador de Gibbs para gerar $\lambda$.

\subsubsection{Densidade Condicional de $k_{1}$ para o Algoritmo amostrador de Gibbs}

A densidade condicional de $\mathrm{k}_{1}$ é obtida através da densidade a posteriori conjunta (3.4.3), considerando $\mathrm{k}_{1}$ condicionado aos dados e ao parâmetro $\lambda$. Assim, temos,

$$
\pi\left(\mathrm{k}_{1} \backslash \lambda, \mathrm{m}_{1}, \mathrm{~m}_{2}, \ldots, \mathrm{m}_{\mathrm{n}}\right) \propto \mathrm{k}_{1}^{\sum_{\mathrm{i}=1}^{\mathrm{n}}(\mathrm{i}-1) \mathrm{m}_{\mathrm{i}}+\mathrm{c}_{1}-1}\left(1-\mathrm{k}_{1}\right)^{\mathrm{c}_{2}-1} \exp \left(-\lambda \sum_{\mathrm{i}=1}^{\mathrm{n}} \mathrm{k}_{1}^{\mathrm{i}-1}\right)
$$

onde $\lambda>0$ e $0<\mathrm{k}_{1}<1$.

Note-se que a distribuição condicional de $\mathrm{k}_{1}$ (3.4.5) não tem uma forma padronizada, ou seja, não tem distribuição de probabilidade conhecida. 
Como comentamos na seção (3.3), quando isto ocorre, utilizamos o algoritmo de Metropolis-Hastings para gerar o parâmetro $\mathrm{k}_{1}$.

Chib e Greenberg (1995) exploram a densidade a posteriori dos parâmetros de interesse, para determinar o núcleo de transição da cadeia de Markov. Por exemplo, se a densidade condicional $\pi(\mathrm{t})$ pode ser escrita como $\pi(\mathrm{t}) \propto \mathrm{h}(\mathrm{t}) \Psi(\mathrm{t})$, onde $h(t)$ é uma densidade com forma padronizada e $\Psi(t)$ é uma função uniformemente limitada, usamos $\mathrm{h}(\mathrm{t})$ como núcleo para gerar os candidatos do parâmetro de interesse.

Assim, para gerar $\mathrm{k}_{1}$ pelo algoritmo de Metropolis-Hastings, primeiramente tentamos identificar um núcleo de transição $\mathrm{h}(\mathrm{t})$ com forma padronizada, o qual é utilizado para gerar candidatos para o parâmetro $\mathrm{k}_{1}$. Neste caso, através da equação (3.4.5), é identificado um núcleo com a forma da distribuição Beta $\left(c_{1}, c_{2}\right)$. Portanto a densidade condicional de $\mathrm{k}_{1}$ dados $\lambda$, e $\mathrm{m}_{1}, \mathrm{~m}_{2}, \ldots, \mathrm{m}_{\mathrm{n}}$, pode ser reescrita por,

$$
\pi\left(\mathrm{k}_{1} \backslash \lambda, \mathrm{m}_{1}, \mathrm{~m}_{2}, \ldots, \mathrm{m}_{\mathrm{n}}\right) \propto \mathrm{k}_{1}^{\mathrm{c}_{1}-1}\left(1-\mathrm{k}_{1}\right)^{\mathrm{c}_{2}-1} \Psi_{1}\left(\lambda, \mathrm{k}_{1}\right),
$$

onde $\Psi_{1}\left(\lambda, \mathrm{k}_{1}\right)=\exp \left\{\sum_{\mathrm{i}=1}^{\mathrm{n}}(\mathrm{i}-1) \mathrm{m}_{\mathrm{i}} \ln \mathrm{k}_{1}-\lambda \sum_{\mathrm{i}=1}^{\mathrm{n}} \mathrm{k}_{1}^{\mathrm{i}-1}\right\}$

Observe que, se é satisfeita a reversibilidade da cadeia de Markov, a probabilidade de movimento com que $\mathrm{k}_{1}{ }^{(\mathrm{s}-1)}$ move-se para $\mathrm{k}_{1}{ }^{(\mathrm{s})}$; requer o cálculo somente da função $\Psi$ (não requer o cálculo de $\pi$ ou h) e é dada por,

$$
\operatorname{minimo}\left\{\frac{\Psi_{1}\left(\lambda^{(\mathrm{s})}, \mathrm{k}_{1}^{(\mathrm{s})}\right)}{\Psi_{1}\left(\lambda^{(\mathrm{s})}, \mathrm{k}_{1}^{(\mathrm{s}-1)}\right)}, 1\right\}
$$

onde $\Psi_{1}\left(\lambda, \mathrm{k}_{1}\right)=\exp \left\{\sum_{\mathrm{i}=1}^{\mathrm{n}}(\mathrm{i}-1) \mathrm{m}_{\mathrm{i}} \ln \mathrm{k}_{1}-\lambda \sum_{\mathrm{i}=1}^{\mathrm{n}} \mathrm{k}_{1}^{\mathrm{i}-1}\right\}$ 
Em caso contrário, permanecemos com $\mathrm{k}_{1}{ }^{(\mathrm{s}-1)}$ (onde $\mathrm{s}$ denota s-ésima simulação).

\subsubsection{Densidade Preditiva e Momentos a Posteriori}

As amostras geradas pelos algoritmos amostrador de Gibbs e MetropolisHastings podem ser utilizadas para fazer inferências sobre os parâmetros dos modelos de confiabilidade de software ou funções dos mesmos. Assim, podemos aproximar os momentos a posteriori de interesse, ou estimar a densidade preditiva para $\mathrm{m}_{\mathrm{i}}$ dado $\mathrm{m}_{1}, \mathrm{~m}_{2}, \ldots, \mathrm{m}_{\mathrm{i}-1}$, utilizando as amostras geradas por esse algoritmo.

A densidade preditiva para a observação futura $\mathrm{m}_{\mathrm{i}}$, condicionada a $\mathrm{m}_{1}, \mathrm{~m}_{2}, \ldots, \mathrm{m}_{\mathrm{i}-1}$, é dada por

$$
\mathrm{f}\left(\mathrm{m}_{\mathrm{i}} \backslash \mathrm{m}_{1}, \ldots \mathrm{m}_{\mathrm{i}-1}\right)=\int_{0}^{1} \int_{0}^{\infty} \mathrm{f}\left(\mathrm{m}_{\mathrm{i}} \backslash \lambda, \mathrm{k}_{1}\right) \pi\left(\lambda, \mathrm{k}_{1} \backslash \mathrm{m}_{1}, \ldots, \mathrm{m}_{\mathrm{i}-1}\right) \mathrm{d} \lambda \mathrm{dk}_{1}
$$

onde $\mathrm{f}\left(\mathrm{m}_{\mathrm{i}} \backslash \lambda, \mathrm{k}_{1}\right)=\frac{\exp \left(-\lambda \mathrm{k}_{1}^{\mathrm{i}-1}\right)\left(\lambda \mathrm{k}_{1}^{\mathrm{i}-1}\right)^{\mathrm{m}_{\mathrm{i}}}}{\mathrm{m}_{\mathrm{i}} !}$

Um estimador Monte-Carlo para $\mathrm{f}\left(\mathrm{m}_{\mathrm{i}} \backslash \mathrm{m}_{1}, \ldots, \mathrm{m}_{\mathrm{i}-1}\right)$ (3.4.7), considerandose $\mathrm{S}$ amostras geradas pelo algoritmo Gibbs com Metrópolis Hastings, é dado por,

$$
\hat{f}\left(m_{i} \backslash m_{1}, \ldots, m_{i-1}\right)=\frac{1}{S} \sum_{s=1}^{s} \frac{e^{-\lambda^{(s)} k_{1}^{(s)^{i-1}}\left(\lambda^{(s)} k_{1}^{(s)^{i-1}}\right)^{m_{i}}}}{m_{i} !}
$$

Um estimador Bayesiano para a taxa de falhas (2.1.1), do modelo de Moranda. com respeito à função de perda quadrática, é dado por:

$$
\mathrm{E}\left(\lambda_{\mathrm{i}} \backslash \mathrm{m}_{1}, \mathrm{~m}_{2}, \ldots, \mathrm{m}_{\mathrm{n}}\right)=\int_{0}^{1} \int_{0}^{\infty} \lambda \mathrm{k}_{1}^{\mathrm{i}-1} \pi\left(\lambda, \mathrm{k}_{1} \backslash \mathrm{m}_{1}, \mathrm{~m}_{2}, \ldots, \mathrm{m}_{\mathrm{n}}\right) \mathrm{d} \lambda \mathrm{dk}_{1},
$$


onde $\pi\left(\lambda, \mathrm{k}_{1} \backslash \mathrm{m}_{1}, \mathrm{~m}_{2}, \ldots, \mathrm{m}_{\mathrm{n}}\right)$ é a densidade a posteriori conjunta de $\lambda \mathrm{e} \mathrm{k}_{1}$ dada em (3.4.2).

Sejam $\lambda^{(\mathrm{r}, \mathrm{s})} \mathrm{e}_{1}^{(\mathrm{r}, \mathrm{s})}$ as variáveis para $\lambda$ e $\mathrm{k}_{1}$, obtidas na r-ésima réplica e $\mathrm{s}$ ésima iteração de $\lambda$ e $\mathrm{k}_{1}$, onde $\mathrm{R}$ e $\mathrm{S}$ são, respectivamente, o número total de cadeias (réplica) e as simulações do amostrador de Gibbs.

Um estimador Monte Carlo para (3.4.9), considerando as amostras geradas pelos algoritmos de Gibbs e Metropolis-Hastings, é dado por,

$$
\hat{\lambda}_{\mathrm{i}}=\frac{2}{\mathrm{RS}} \sum_{\mathrm{r}=1}^{\mathrm{R}} \sum_{\mathrm{s}=\frac{\mathrm{S}}{2}+1}^{\mathrm{S}} \lambda^{(\mathrm{r}, \mathrm{s})} \mathrm{k}_{1}^{(\mathrm{r}, \mathrm{s})^{\mathrm{i}-1}}
$$

Com as aproximações das densidades a posteriori de interesse, momentos $a$ posteriori e densidades preditivas, utilizando as amostras aleatórias geradas pelo amostrador de Gibbs e Metropolis-Hastings, verificamos a simplicidade em utilizar esses algoritmos computacionais, quando temos interesse em fazer inferências para os parâmetros dos modelos de confiabilidade de software ou funções dos mesmos.

\subsection{Análise Bayesiana da Generalização do Modelo de Moranda Considerando Densidades a Priori Informativas}

Apresentamos a seguir uma análise Bayesiana da forma generalizada do Modelo de Moranda, com função intensidade (2.2.1), considerando densidades $a$ priori informativas. Utilizamos os algoritmos amostrador de Gibbs e MetropolisHastings para aproximar as integrais a posteriori de interesse.

\subsubsection{Densidades $\boldsymbol{a}$ Priori Informativas para $\lambda_{\mathrm{a}}, \mathrm{k}_{1}$ e $\mathrm{k}_{2}$}

Assumindo que temos informações a respeito dos parâmetros $\lambda_{\mathrm{a}}, \mathrm{k}_{1}$ e $\mathrm{k}_{2}$, através de opinião de especialistas de software ou de estudos preliminares dos 
dados de falhas, vamos considerar densidades a priori informativas para os parâmetros $\lambda_{\mathrm{a}}, \mathrm{k}_{1}$ e $\mathrm{k}_{2}$.

Supondo independência para os parâmetros $\lambda_{\mathrm{a}}, \mathrm{k}_{1} \mathrm{e} \mathrm{k}_{2}$, temos as seguintes densidades a priori:

(i) $\lambda_{\mathrm{a}} \sim \Gamma\left(\mathrm{b}_{1}, \mathrm{~b}_{2}\right)\left(\right.$ para $\left.\lambda_{\mathrm{a}}>0\right)$,

(ii) $\mathrm{k}_{1} \sim \mathrm{B}\left(\mathrm{c}_{1}, \mathrm{c}_{2}\right)\left(\right.$ para $\left.0<\mathrm{k}_{1}<1\right)$,

(iii) $\mathrm{k}_{2} \sim \mathrm{N}\left(\mu_{0}, \sigma_{0}^{2}\right)\left(\right.$ para $\left.-\infty<\mathrm{k}_{2}<\infty\right)$,

onde $\Gamma\left(\mathrm{b}_{1}, \mathrm{~b}_{2}\right)$ denota a distribuição gama, $\mathrm{B}\left(\mathrm{c}_{1}, \mathrm{c}_{2}\right)$ denota a distribuição beta, ambas definidas em (3.4.2), e $\mathrm{N}\left(\mu_{0}, \sigma_{0}^{2}\right)$ denota a distribuição normal com média $\mu_{0}$ e variância $\sigma_{0}^{2}$.

\subsubsection{Densidade a Posteriori Conjunta para $\lambda_{\mathrm{a}}, \mathrm{k}_{1}$ e $\mathrm{k}_{2}$}

A densidade a posteriori conjunta para $\lambda_{\mathrm{a}}, \mathrm{k}_{1}$ e $\mathrm{k}_{2}$, considerando-se as densidades a priori (3.5.1) e a função de verossimilhança (2.2.2), é dada por:

$$
\begin{aligned}
& \pi\left(\lambda_{\mathrm{a}}, \mathrm{k}_{1}, \mathrm{k}_{2} \backslash \mathrm{m}_{1}, \mathrm{~m}_{2}, \ldots, \mathrm{m}_{\mathrm{n}}\right) \propto \lambda_{\mathrm{a}}^{\mathrm{d}_{1}+\mathrm{b}_{1}-1} \mathrm{e}^{-\left[\mathrm{b}_{2}+\mathrm{A}\left(\mathrm{k}_{1}, \mathrm{k}_{2}\right)\right] \lambda_{\mathrm{a}}} \\
& \mathrm{k}_{1}^{\mathrm{d}_{2}\left(\mathrm{k}_{2}\right)+\mathrm{c}_{1}-1}\left(1-\mathrm{k}_{1}\right)^{\mathrm{c}_{2}-1} \exp \left\{-\frac{1}{2 \sigma_{0}^{2}}\left(\mathrm{k}_{2}-\mu_{0}\right)^{2}\right\},
\end{aligned}
$$

onde $\lambda_{\mathrm{a}}>0$, e $0<\mathrm{k}_{1}<1$ e $-\infty<\mathrm{k}_{2}<\infty, \mathrm{d}_{2}\left(\mathrm{k}_{2}\right)=\sum_{\mathrm{i}=1}^{\mathrm{n}} \mathrm{i}^{\mathrm{k}_{2}} \mathrm{~m}_{\mathrm{i}}$

$\mathrm{e} A\left(\mathrm{k}_{1}, \mathrm{k}_{2}\right)=\sum_{\mathrm{i}=1}^{\mathrm{n}} \mathrm{k}_{1}^{\mathrm{i}^{\mathrm{k}} \mathrm{k}_{2}}$ 
3.5.2.1 Densidade Condicional de $\lambda_{\mathrm{a}}$ para o Algoritmo Amostrador de Gibbs

A densidade condicional de $\lambda_{\mathrm{a}}$, condicionada aos dados, $\mathrm{m}_{1}, \ldots, \mathrm{m}_{\mathrm{n}}$ e aos parâmetros $\mathrm{k}_{1} \mathrm{e} \mathrm{k}_{2}$, é obtida através da densidade a posteriori conjunta (3.5.2), dada por:

$$
\pi\left(\lambda_{\mathrm{a}} \backslash \mathrm{k}_{1}, \mathrm{k}_{2}, \mathrm{~m}_{1}, \ldots, \mathrm{m}_{\mathrm{n}}\right) \sim \Gamma\left(\mathrm{d}_{1}+\mathrm{b}_{1}, \mathrm{~b}_{2}+\mathrm{A}\left(\mathrm{k}_{1}, \mathrm{k}_{2}\right)\right)
$$

onde $\mathrm{A}\left(\mathrm{k}_{1}, \mathrm{k}_{2}\right)=\sum_{\mathrm{i}=1}^{\mathrm{n}} \mathrm{k}_{1}^{\mathrm{i}^{\mathrm{k}}}$ e $\lambda_{\mathrm{a}}>0$, e $0<\mathrm{k}_{1}<1$ e $-\infty<\mathrm{k}_{2}<\infty$.

A densidade condicional de $\lambda_{\mathrm{a}}$ (3.5.3) tem forma padronizada gama. Portanto, geramos $\lambda_{\mathrm{a}}$ utilizando o algoritmo amostrador de Gibbs.

\subsubsection{Densidade Condicional de $\mathrm{k}_{1}$ para o Algoritmo de Gibbs}

A densidade de $\mathrm{k}_{1}$, condicionada aos dados, $\mathrm{m}_{1}, \ldots, \mathrm{m}_{\mathrm{n}}, \lambda_{\mathrm{a}}$ e $\mathrm{k}_{2}$, é dada por:

$$
\begin{aligned}
& \pi\left(\mathrm{k}_{1} \backslash \lambda_{\mathrm{a}}, \mathrm{k}_{2}, \mathrm{~m}_{1}, \ldots, \mathrm{m}_{\mathrm{n}}\right) \propto \mathrm{k}_{1}^{\mathrm{d}_{2}\left(\mathrm{k}_{2}\right)+\mathrm{c}_{1}-1}\left(1-\mathrm{k}_{1}\right)^{\mathrm{c}_{2}-1} \\
& \exp \left\{-\lambda_{\mathrm{a}} \mathrm{A}\left(\mathrm{k}_{1}, \mathrm{k}_{2}\right)\right\}
\end{aligned}
$$

$$
\begin{aligned}
& \text { onde } \mathrm{d}_{2}\left(\mathrm{k}_{2}\right)=\sum_{\mathrm{i}=1}^{\mathrm{n}} \mathrm{i}^{\mathrm{k}_{2}} \mathrm{~m}_{\mathrm{i}}, \mathrm{A}\left(\mathrm{k}_{1}, \mathrm{k}_{2}\right)=\sum_{\mathrm{i}=1}^{\mathrm{n}} \mathrm{k}_{1}^{\mathrm{i}^{\mathrm{k}}}, \lambda_{\mathrm{a}}>0,0<\mathrm{k}_{1}<1 \\
& \text { e }-\infty<\mathrm{k}_{2}<\infty
\end{aligned}
$$

Atente-se que a densidade condicional de $\mathrm{k}_{1}$ (3.5.4) não tem forma padronizada, portanto, utilizamos o algoritmo de Metropolis-Hastings para gerar este parâmetro. 
Como comentamos na seção (3.4.2.2), a densidade condicional (3.5.4) pode ser reescrita na forma,

$$
\pi\left(\mathrm{k}_{1} \backslash \lambda_{\mathrm{a}}, \mathrm{k}_{2}, \mathrm{~m}_{1}, \ldots, \mathrm{m}_{\mathrm{n}}\right) \propto \mathrm{k}_{1}^{\mathrm{c}_{1}-1}\left(1-\mathrm{k}_{1}\right)^{\mathrm{c}_{2}-1} \Psi_{2}\left(\lambda_{\mathrm{a}}, \mathrm{k}_{1}, \mathrm{k}_{2}\right),
$$

onde $\Psi_{2}\left(\lambda_{\mathrm{a}}, \mathrm{k}_{1}, \mathrm{k}_{2}\right)=\exp \left\{-\lambda_{\mathrm{a}} \mathrm{A}\left(\mathrm{k}_{1}, \mathrm{k}_{2}\right)+\mathrm{d}_{2}\left(\mathrm{k}_{2}\right) \log \left(\mathrm{k}_{1}\right)\right\}$.

$\mathrm{O}$ núcleo de transição da cadeia $\mathrm{h}(\mathrm{t})$ tem forma conhecida, dada pela distribuição $\operatorname{Beta}\left(c_{1}, c_{2}\right)$. Portanto, a distribuição candidata para gerar $k_{1}$ é a distribuição $\operatorname{Beta}\left(c_{1}, c_{2}\right)$.

\subsubsection{Densidade Condicional de $\mathrm{k}_{2}$ para o Algoritmo de Gibbs}

A densidade condicional de $\mathrm{k}_{2}$ para o algoritmo de Gibbs, condicionado aos dados $\left(\mathrm{m}_{1}, \ldots, \mathrm{m}_{\mathrm{n}}\right)$ e aos parâmetros $\lambda_{\mathrm{a}}$ e $\mathrm{k}_{1}$ é dada por,

$$
\begin{aligned}
& \pi\left(\mathrm{k}_{2} \backslash \mathrm{k}_{1}, \lambda_{\mathrm{a}}, \mathrm{m}_{1}, \ldots, \mathrm{m}_{\mathrm{n}}\right) \propto \mathrm{k}_{1}^{\mathrm{d}_{2}\left(\mathrm{k}_{2}\right)} \exp \left\{-\lambda_{\mathrm{a}} \mathrm{A}\left(\mathrm{k}_{1}, \mathrm{k}_{2}\right)\right\} \\
& \exp \left\{-\frac{1}{2 \sigma^{2}}\left(\mathrm{k}_{2}-\mu_{0}\right)^{2}\right\}
\end{aligned}
$$

onde $\mathrm{d}_{2}\left(\mathrm{k}_{2}\right)=\sum_{\mathrm{i}=1}^{\mathrm{n}} \mathrm{i}^{\mathrm{k}_{2}} \mathrm{~m}_{\mathrm{i}}, \mathrm{A}\left(\mathrm{k}_{1}, \mathrm{k}_{2}\right)=\sum_{\mathrm{i}=1}^{\mathrm{n}} \mathrm{k}_{1}^{\mathrm{i}^{\mathrm{k}}}, \lambda_{\mathrm{a}}>0,0<\mathrm{k}_{1}<1$
e $-\infty<\mathrm{k}_{2}<\infty$

Observe que a densidade condicional de $\mathrm{k}_{2}$ (3.5.6) não tem forma conhecida, portanto, utilizamos o algoritmo Metropolis-Hastings para gerar $\mathrm{k}_{2}$.

Assim, (3.5.6) pode ser reescrita na forma:

$$
\pi\left(\mathrm{k}_{2} \backslash \mathrm{k}_{1}, \lambda_{\mathrm{a}}, \mathrm{m}_{1}, \ldots, \mathrm{m}_{\mathrm{n}}\right) \propto \exp \left\{-\frac{1}{2 \sigma^{2}}\left(\mathrm{k}_{2}-\mu_{0}\right)^{2}\right\} \Psi_{3}\left(\lambda_{\mathrm{a}}, \mathrm{k}_{1}, \mathrm{k}_{2}\right),
$$


onde $\Psi_{3}\left(\lambda_{\mathrm{a}}, \mathrm{k}_{1}, \mathrm{k}_{2}\right)=\exp \left\{\mathrm{d}_{2}\left(\mathrm{k}_{2}\right) \log \left(\mathrm{k}_{1}\right)-\lambda_{\mathrm{a}} \mathrm{A}\left(\mathrm{k}_{1}, \mathrm{k}_{2}\right)\right\}$.

$\mathrm{O}$ núcleo de transição usado para gerar $\mathrm{k}_{2}$ é a distribuição normal $\mathrm{N}\left(\mu_{0}, \sigma_{0}^{2}\right)$ com média $\mu_{0}$ e variância $\sigma_{0}^{2}$.

\subsubsection{Momentos a Posteriori e Densidade Preditiva}

Admitindo-se $\lambda_{\mathrm{a}}^{(\mathrm{r}, \mathrm{s})}, \mathrm{k}_{1}^{(\mathrm{r}, \mathrm{s})}$ e $\mathrm{k}_{2}^{(\mathrm{r}, \mathrm{s})}$ como as variáveis geradas pelo algoritmo de Gibbs para $\lambda_{a}, k_{1}$ e $k_{2}$ obtidas na r-ésima réplica (cadeia) e s-ésima iteração, um estimador Monte Carlo para a função intensidade (2.2.1) é dado por:

$$
\hat{\lambda}_{i}=\frac{2}{\operatorname{RS}} \sum_{\mathrm{r}=1}^{\mathrm{R}} \sum_{\mathrm{s}=\frac{\mathrm{S}}{2}+1}^{\mathrm{S}} \lambda_{\mathrm{a}}^{(\mathrm{r}, \mathrm{s})} \mathrm{k}_{1}^{(\mathrm{r}, \mathrm{s})^{\mathrm{k}_{2}^{(\mathrm{r}, \mathrm{s})}}}
$$

Para a generalização do modelo de Moranda, a densidade preditiva para a observação futura $\mathrm{m}_{\mathrm{i}}$ condicionada a $\mathrm{m}_{1}, \mathrm{~m}_{2}, \ldots, \mathrm{m}_{\mathrm{i}-1}$, $(\mathrm{i}=2,3, \ldots)$ é definida por,

$\mathrm{f}\left(\mathrm{m}_{\mathrm{i}} \backslash \mathrm{m}_{1}, \ldots, \mathrm{m}_{\mathrm{i}-1}\right)=\int_{0}^{\infty} \int_{-\infty}^{\infty} \int_{0}^{1} \mathrm{f}\left(\mathrm{m}_{\mathrm{i}} \backslash \lambda_{\mathrm{a}}, \mathrm{k}_{1}, \mathrm{k}_{2}\right) \pi\left(\lambda_{\mathrm{a}}, \mathrm{k}_{1}, \mathrm{k}_{2} \backslash \mathrm{m}_{1}, \ldots, \mathrm{m}_{\mathrm{i}-1}\right) \mathrm{dk}_{1} \mathrm{dk}_{2} \mathrm{~d} \lambda_{\mathrm{a}}$

onde $\mathrm{f}\left(\mathrm{m}_{\mathrm{i}} \backslash \lambda_{\mathrm{a}}, \mathrm{k}_{1}, \mathrm{k}_{2}\right)=\frac{\exp \left(-\lambda_{\mathrm{a}} \mathrm{k}_{1}^{\mathrm{k}_{2}}\right)\left(\lambda_{\mathrm{a}} \mathrm{k}_{1}^{\mathrm{i}_{2}}\right)^{\mathrm{m}_{\mathrm{i}}}}{\mathrm{m}_{\mathrm{i}} !}$.

Utilizando as amostras geradas pelo algoritmo amostrador de Gibbs (ver apêndice B), a densidade preditiva (3.5.9) para uma observação futura $\mathrm{m}_{\mathrm{i}}$, condicionada a $\mathrm{m}_{1}, \mathrm{~m}_{2}, \ldots, \mathrm{m}_{\mathrm{i}-1}$, pode ser aproximada por um estimador Monte Carlo dado por,

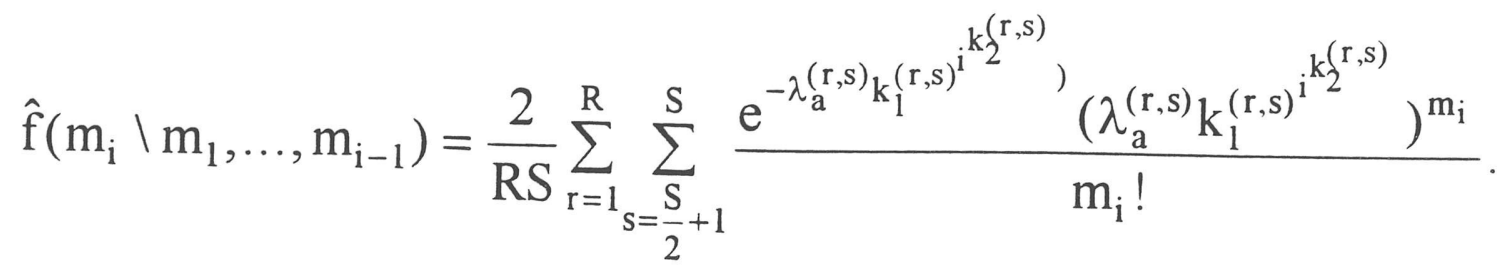




\section{Capítulo 4}

\section{PROCESSOS DE POISSON NÃO HOMOGÊNEOS UM ENFOQUE BAYESIANO}

Muitos modelos de confiabilidade crescente têm sido propostos para modelar os tempos de falhas de um software em teste. Estes modelos incluem a classe de modelos de estatísticas de ordem proposta por Yang (1994). Neste capítulo, propomos uma extensão dos modelos de estatísticas de ordem, assumindo a distribuição gaussiana inversa para modelar a função de valor médio $m(t)$ dos processos de Poisson não homogêneos (ver Achcar e Storani, 1996). Além disso, consideramos os modelos de estatísticas de ordem exponencial (ver Goel e Okumoto, 1979), gama (ver Yamada, Ohba e Osaki, 1983 ou Kuo, Lee, Choi e Yang, 1996), Weibul (ver Goel, 1983), gama generalizada e log-normal (ver Achcar Dey e Niverthy, 1996) e fazemos, para esses modelos, uma análise Bayesiana. Consideramos densidades a priori informativas e não-informativas. Algoritmos computacionais como o amostrador de Gibbs e Metropolis-Hastings (ver Gelfand e Smith, 1990 ou Chib e Greenberg, 1995) são utilizados para implementar os cálculos Bayesianos. A técnica de dados aumentados (ver Tanner e Young, 1987), com a inclusão de uma variável latente (ver Yang, 1994), é utilizada para especificar as densidades condicionais do algoritmo amostrador de Gibbs.

$\mathrm{Na}$ seção 4.1, apresentamos a função de verossimilhança dos processos de Poisson não homogêneos e mostramos a relação entre os modelos de estatísticas de ordem e os modelos PPNH. Além disso, introduzimos os modelos de estatísticas de ordem considerados nesta dissertação. Na seção 4.2, apresentamos as densidades condicionais dos parâmetros de interesse para o algoritmo amostrador de Gibbs e Metropolis Hastings. 


\subsection{Modelos de Confiabilidade de Software com Processo de Poisson Não Homogêneo}

Seja $M(t)$ o número de falhas de um software observadas no intervalo $(0, t]$ e $E(M(t))=m(t)$.

Para a modelagem de $\mathrm{M}(\mathrm{t})$, podemos considerar duas classes diferentes de modelos: (1) modelos de estatísticas de ordem em geral $\left(\right.$ se $\left.\lim _{\mathrm{t} \rightarrow \infty} \mathrm{m}(\mathrm{t})<\infty\right)$; (2) modelos de estatísticas de valores observados (se $\mathrm{m}(\mathrm{t}) \rightarrow \infty$, quando $\mathrm{t} \rightarrow \infty$ ) (ver Yang, 1994).

Para ambas as classes de modelos, $\mathrm{M}(\mathrm{t})$ pode ser modelado por um processo de Poisson não homogêneo.

Neste trabalho, consideramos a classe dos modelos de estatísticas de ordem para modelar $\mathrm{M}(\mathrm{t})$.

\subsubsection{Modelos de Estatísticas de Ordem}

Os modelos de estatísticas de ordem assumem que existe um número desconhecido $\mathrm{N}$ de erros no início do teste do software. Os instantes de falhas são as n primeiras estatísticas de ordem i.i.d. tomadas de $\mathrm{N}$, com função densidade $f$, limitada em $\mathfrak{R}^{+}$. Os modelos de estatísticas de ordem supõem que $\mathrm{N}$ tem distribuição de Poisson com média $\theta$. Então, pode-se mostrar que $\mathrm{m}(\mathrm{t})=\theta \mathrm{F}(\mathrm{t})$, onde $\mathrm{F}$ é a função distribuição com densidade $f$. Observe-se que $\lim _{\mathrm{t} \rightarrow \infty} \mathrm{m}(\mathrm{t})=\theta$, denotado por PPNH-I. Se $\mathrm{F}(\mathrm{t})=1-\mathrm{e}^{-\beta \mathrm{t}}$ (função distribuição exponencial), então $\mathrm{M}(\mathrm{t})$ é um PPNH-I com função de valor médio $m(t)=\theta\left(1-e^{-\beta t}\right)$. Este é o processo de Goel e Okumoto (1979). Outros processos podem ser considerados, por variação de F. Por exemplo: Weibul PPNH-I, quando F é a função distribuição Weibul; e gama PPNH-I quando $\mathrm{F}$ é a função distribuição gama.

$\mathrm{Na}$ inferência para os modelos de estatísticas de ordem , Raftery (1987) apresenta uma análise Bayesiana empírica; Joe (1989) desenvolve resultados assintóticos para estimadores de máxima verossimilhança; Yang (1994), e Achcar, Dey e Niverthy (1996) desenvolvem inferência Bayesiana para os PPNH-I. Utilizam a técnica de dados aumentados com a inclusão da 
variável latente, número de erros remanescentes no software, com o objetivo de facilitar a especificação das densidades condicionais usadas no amostrador de Gibbs com Metropolis.

A seguir, apresentamos a função de verossimilhança dos PPNH e mostramos a relação existente entre os modelos de estatísticas de ordem e os modelos PPNH-I.

Vamos supor $m(t)$ indexado por parâmetros desconhecidos $\theta$ e $\beta$, onde $\theta$ é um número real não negativo e $\beta$ um vetor de parâmetros desconhecidos. Para simplificação de notação, escrevemos $m(t)=m(t \backslash \theta, \beta)$.

Considerando-se o modelo de tempo truncado (ver Cox e Lewis, 1966 ou Lawless, 1982), testado até o tempo t, os instantes das $n$ falhas observado no intervalo $(0, t]$, são denotados por $x_{1}, x_{2}, \ldots, x_{n}$.

Neste capítulo, o conjunto de dados é escrito por $D_{t}=\left\{n, x_{1}, \ldots, x_{n} ; t\right\}$.

\subsubsection{Função de verossimilhança}

Nesta seção apresentamos dois métodos para obter a função de verossimilhança dos modelos com processos de Poisson não homogêneos. Método 1, utiliza um argumento condicional, $\mathrm{M}(\mathrm{t})=\mathrm{n}$ (ver Musa, Yannino e Okumoto, 1987 ou Yang, 1994). Método 2, transforma um PPNH por um processo de Poisson homogêneo.

Método 1: Tomando o modelo de tempo truncado, o número de falhas observadas em $(0, \mathrm{t}]$ é uma variável aleatória, denotada por $\mathrm{n}$.

Dado $M(t)=n$, os instantes de falhas observados no intervalo de tempo $(0, \mathrm{t}] \mathrm{x}_{1}<\ldots<\mathrm{x}_{\mathrm{n}}<\mathrm{t}$, são estatísticas de ordem (ver Mood, Graybill e Boes, 1974) de variáveis aleatórias i.i.d, com função densidade de probabilidade dada por: 


$$
\mathrm{f}\left(\mathrm{x}_{\mathrm{i}}\right)=\frac{\lambda\left(\mathrm{x}_{\mathrm{i}}\right)}{\mathrm{m}(\mathrm{t})}, \quad 0 \leq \mathrm{x}_{\mathrm{i}} \leq \mathrm{t}
$$

Portanto, a função de verossimilhança condicionada aos dados, para o modelo de tempo truncado, é definida por,

$$
\begin{aligned}
\underset{\sim}{L}\left(\theta, \beta \backslash D_{t}\right)= & p\left(D_{t} \backslash M(t)=n\right) \times p(M(t)=n) \\
& =n !\left[\prod_{i=1}^{n} \frac{\lambda\left(x_{i}\right)}{m(t)}\right] \frac{e^{-m(t)} m(t)^{n}}{n !},
\end{aligned}
$$

ou seja,

$$
\underset{\sim}{L}\left(\theta, \beta \backslash D_{t}\right)=\prod_{i=1}^{n} \lambda\left(x_{i}\right) \exp \{-m(t)\}
$$

Para o modelo de falha truncado, uma expressão similar para (4.1.1) pode ser obtida com $t$ substituído por $\mathrm{x}_{\mathrm{n}}$.

Método 2: Transformando um processo de Poisson não homogêneo para um processo de Poisson homogêneo, pode-se mostrar que $\mathrm{m}\left(\mathrm{x}_{1}\right)<\mathrm{m}\left(\mathrm{x}_{2}\right)<\ldots$ são os tempos de chegadas no processo de Poisson homogêneo com taxa de falha 1 (Cinlar 1975, pág. 97). Os tempos entre chegadas no processo de Poisson homogêneo são variáveis aleatórias exponenciais com taxa de falha 1 , e os eventos $\left[m\left(X_{n}\right) \geq m\left(X_{n-1}+t\right)\right]$ e $\left[X_{n}-X_{n-1}>t\right]$ são equivalentes, assim podemos provar que,

$$
\begin{aligned}
\mathrm{p}\left(\mathrm{X}_{\mathrm{n}}-\mathrm{X}_{\mathrm{n}-1}\right. & \left.>\mathrm{t} \backslash X_{1}, \ldots, X_{\mathrm{n}-1}\right)=\mathrm{p}\left(\mathrm{m}\left(\mathrm{X}_{\mathrm{n}}\right) \geq \mathrm{m}\left(\mathrm{t}+\mathrm{X}_{\mathrm{n}-1}\right) \backslash X_{1}, \ldots, X_{\mathrm{n}-1}\right) \\
& =\exp \left[-\mathrm{m}\left(X_{\mathrm{n}-1}+\mathrm{t}\right)+\mathrm{m}\left(X_{\mathrm{n}-1}\right)\right]=\mathrm{p}\left(X_{\mathrm{n}}-X_{\mathrm{n}-1}>\mathrm{t} \backslash X_{\mathrm{n}-1}\right) .
\end{aligned}
$$

Além disso,

$$
\begin{gathered}
f_{\mathrm{X}_{1}}\left(\mathrm{x}_{1}\right)=\lambda\left(\mathrm{x}_{1}\right) \exp \left(-\mathrm{m}\left(\mathrm{x}_{1}\right)\right) \\
f_{\mathrm{X}_{1} \backslash \mathrm{X}_{\mathrm{n}-1}}\left(\mathrm{x}_{\mathrm{n}} \backslash \mathrm{x}_{\mathrm{n}-1}\right)=\lambda\left(\mathrm{x}_{\mathrm{n}}\right) \exp \left[-\mathrm{m}\left(\mathrm{x}_{\mathrm{n}}\right)+\mathrm{m}\left(\mathrm{x}_{\mathrm{n}-1}\right)\right] .
\end{gathered}
$$


Portanto, a função de verossimilhança para o modelo de tempo truncado é dada por,

$$
\begin{aligned}
& \mathrm{L}_{\mathrm{PPNH}}\left(\theta, \beta ; \mathrm{D}_{\mathrm{t}}\right)=f\left(\mathrm{x}_{1}, \ldots, \mathrm{x}_{\mathrm{n}} ; \mathrm{t}\right) \\
& =f_{\mathrm{X}_{1}}\left(\mathrm{x}_{1}\right) f_{\mathrm{X}_{2} \backslash \mathrm{X}_{1}}\left(\mathrm{x}_{2} \backslash \mathrm{x}_{1}\right) \ldots f_{\mathrm{X}_{\mathrm{n}} \backslash \mathrm{x}_{\mathrm{n}-1}}\left(\mathrm{x}_{\mathrm{n}} \backslash \mathrm{x}_{\mathrm{n}-1}\right) \mathrm{p}\left(\mathrm{X}_{\mathrm{n}+1}>\mathrm{t} \backslash \mathrm{x}_{\mathrm{n}}\right) \\
& =\underset{\sim}{L}\left(\theta, \beta \backslash D_{t}\right)=\prod_{i=1}^{n} \lambda\left(x_{i}\right) \exp \{-m(t)\},
\end{aligned}
$$

(ver Cox e Lewis, 1966 ou Yang, 1994).

\subsubsection{Estatísticas de Ordem Genéricas e PPNH-I}

Os modelos de estatísticas de ordem assumem que $\lim _{t \rightarrow \infty} m(t)=\theta$. Portanto, temos os PPNH-I.

Seja N o número de erros no início do teste do software. Então, podemos mostrar que a função de verossimilhança dos modelos de estatísticas de ordem, dada em Raftery (1987) e Joe (1989), é a função de verossimilhança de $\mathrm{N}, \theta$ e $\beta$ nos modelos PPNH-I.

A função de verossimilhança de $\mathrm{N}, \theta$ e $\beta$, condicionados a $D_{t}$ para o modelo de tempo truncado dos PPNH-I, é dada por,

$$
\mathrm{L}_{\mathrm{PPNH}-\mathrm{I}}\left(\theta, \underset{\sim}{\beta, N} \backslash \mathrm{D}_{\mathrm{t}}\right)=\frac{\mathrm{N} !}{(\mathrm{N}-\mathrm{n}) !}\left[\prod_{\mathrm{i}=1}^{\mathrm{n}} \frac{\lambda\left(\mathrm{x}_{\mathrm{i}}\right)}{\theta}\right]\left(1-\frac{\mathrm{m}(\mathrm{t})}{\theta}\right)^{\mathrm{N}-\mathrm{n}} .
$$

Seja $\mathrm{p}(\mathrm{N} \backslash \theta)$, denotando a função densidade de probabilidade de Poisson com média $\theta$. Então, é simples verificar que,

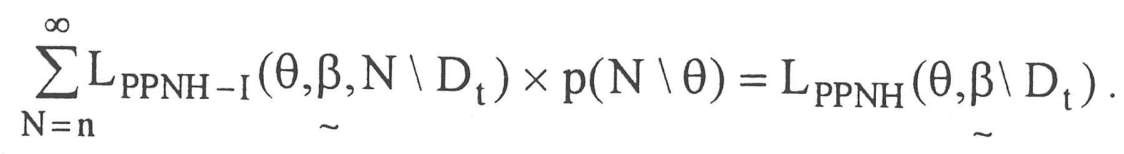


Observe-se que a função de verossimilhança (4.1.1) é a função de verossimilhança marginal, quando $\mathrm{N}$ tem uma distribuição de Poisson.

Considere-se agora o modelo de estatísticas de ordem em geral. A classe desses modelos assume que os instantes de falhas são as n primeiras estatísticas de ordem tomadas de $\mathrm{N}$ observações i.i.d, com função densidade $f(S \backslash \beta)$ e função distribuição $\mathrm{F}(\mathrm{S} \backslash \beta)$.

Assim, a função de verossimilhança do modelo de falha truncado é dada por

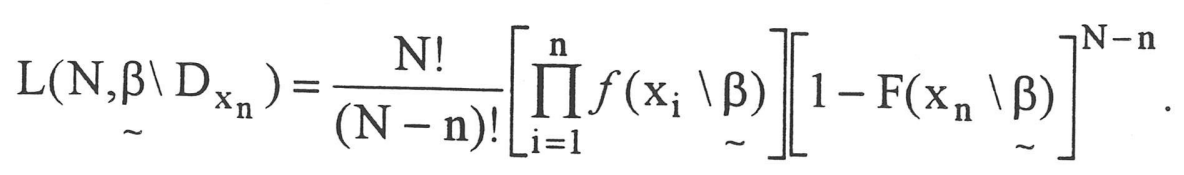

A função de verossimilhança dos modelos de estatísticas de ordem para o modelo de tempo truncado é similar para (4.1.3), exceto o argumento $\mathrm{x}_{\mathrm{n}}$, substituído por $\mathrm{t}$.

Note-se que, se $\mathrm{m}(\mathrm{t})=\theta \mathrm{F}(\mathrm{t})$ (consequentemente, $\lambda(\mathrm{t})=\theta f(\mathrm{t}))$, a função de verossimilhança condicional (4.1.2) é idêntica à função de verossimilhança dos modelos de estatísticas de ordem (4.1.3).

Suponha-se que os instantes de falhas seguem um modelo de estatísticas de ordem e $\mathrm{N}$ tem distribuição de Poisson com média $\theta$; então, podemos mostrar que $\mathrm{M}(\mathrm{t})$ é um PPNH-I com função de valor médio $\mathrm{m}(\mathrm{t})=\theta \mathrm{F}(\mathrm{S} \backslash \beta)$.

Para o modelo de estatísticas de ordem, a probabilidade condicional $\mathrm{P}(\mathrm{M}(\mathrm{t})=\mathrm{n} \backslash \mathrm{N})$ é dada por

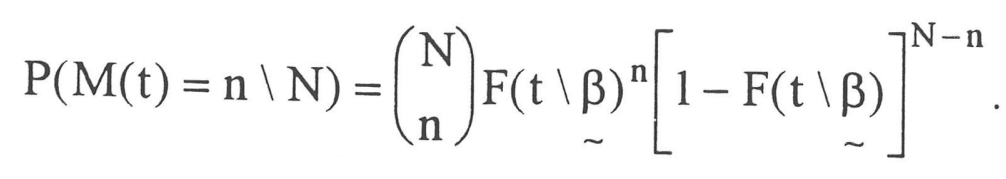


A probabilidade não condicional é dada por

$$
\begin{aligned}
\mathrm{P}(\mathrm{M}(\mathrm{t})=\mathrm{n}) & =\sum_{\mathrm{N}=\mathrm{n}}^{\infty}\left(\begin{array}{l}
\mathrm{N} \\
\mathrm{n}
\end{array}\right) \mathrm{F}(\mathrm{t} \backslash \underset{\sim}{\sim})^{\mathrm{n}}[1-\mathrm{F}(\mathrm{t} \backslash \underset{\sim}{\sim})]^{\mathrm{N}-\mathrm{n}} \frac{\theta^{N} e^{-\theta}}{\mathrm{N} !} \\
& =\frac{\left.\mathrm{e}^{-\theta \mathrm{F}(\mathrm{t} \backslash \beta)}[\mathrm{F}(\mathrm{t} \backslash \beta)]^{\mathrm{n}}\right]}{\mathrm{n} !} .
\end{aligned}
$$

Portanto, M(t) é um PPNH-I com função de valor médio dada por,

$$
m(t)=\theta F(t \backslash \beta)
$$

A seguir, apresentamos os modelos de estatísticas de ordem considerados neste trabalho.

\subsubsection{Modelo de Tempo Dependente e Detecção de Erro (Goel e Okumoto, 1979)}

O modelo de tempo dependente e detecção de erro foi originalmente apresentado por Goel e Okumoto (1979). O seu nome é dado justamente por assumir que os tempos entre sucessivas falhas são dependentes e utilizar um fator de redução de erro na composição do modelo.

Para a construção deste modelo, Goel e Okumoto partiram de algumas suposições:

- O número de erros no software é uma variável aleatória;

- O número de falhas no software no tempo $t$, dado pela função de valor médio $\mathrm{m}(\mathrm{t})$, é não decrescente e limitada acima. Especificamente $\mathrm{m}(0)=0 \mathrm{e}$ $\lim _{t \rightarrow \infty} \mathrm{m}(\mathrm{t})=\theta$, onde $\theta$ representa o número esperado de erros do software.

Os autores assumiram, também, que o número de falhas no tempo ( $t$; $\mathrm{t}+\Delta \mathrm{t}$ ) é proporcional ao número de erros não detectados, isto é,

$$
\mathrm{m}(\mathrm{t}+\Delta \mathrm{t})-\mathrm{m}(\mathrm{t})=\beta(\theta-\mathrm{m}(\mathrm{t})) \Delta \mathrm{t}+\mathrm{o}(\Delta \mathrm{t}),
$$


onde $\beta$ é a taxa de detecção de erro (ou fator de redução de erro) ${ }^{1}$.

Para o modelo de Goel e Okumoto (1979), a função de valor médio é obtida considerando a função distribuição exponencial $F(t \backslash \beta)$ em (4.1.4). Assim, temos,

$$
m(t)=\theta\left(1-e^{-\beta t}\right)
$$

onde $\theta>0$ e $\beta>0$.

Derivando a expressão (4.1.5), obtemos a função intensidade, dada por,

$$
\lambda(\mathrm{t})=\theta \beta \mathrm{e}^{-\beta \mathrm{t}}
$$

\subsubsection{Modelo de Goel (1983)}

Experiências têm mostrado que, de um modo geral, a taxa de falhas do software cresce inicialmente antes de um eventual decréscimo. Considerando este fato, Goel (1983) propôs uma generalização do modelo de Goel e Okumoto (1979). Nesse modelo, a função distribuição, considerada para modelar a função de valor médio (4.1.4), é a distribuição de Weibull. Portanto, a função de valor médio (4.1.4), para o modelo de Goel (1983) é dada por:

$$
m(t)=\theta\left(1-e^{-\beta t^{\alpha}}\right),
$$

onde $\beta>0$ e $\alpha>0$.

${ }^{1} \beta$ é chamado de fator de redução de erro por Musa Iannino e Okumoto (1987) e de taxa de detecção de erro, por Singpurwalla e Wilson (1994). 
Derivando a expressão (4.1.7) obtemos a função intensidade,

$$
\lambda(t)=\theta \beta \alpha t^{\alpha-1} e^{-\beta t^{\alpha}},
$$

onde $\theta$ representa o número esperado de erros no software e os parâmetros $\alpha$ e $\beta$ descrevem a qualidade do teste.

\subsubsection{Modelo de Confiabilidade de Software Tipo Gama}

O modelo de confiabilidade de software Tipo-Gama (ver, por exemplo Yamada, Ohba e Osaki, 1983) assume a função distribuição gama para modelar a função de valor médio (4.1.4) dada por,

$$
\mathrm{m}(\mathrm{t})=\theta\left(1-\mathrm{e}^{-\beta \mathrm{t}} \sum_{\mathrm{j}=0}^{k-1} \frac{(\beta \mathrm{t})^{\mathrm{j}-1}}{\mathrm{j} !}\right)
$$

onde $k>0$ e $\beta>0$.

Derivando (4.1.8), obtemos a função intensidade de falha dada por,

$$
\lambda(\mathrm{t})=\theta \frac{\beta^{k}}{\Gamma(k)} \mathrm{t}^{k-1} \mathrm{e}^{-\beta \mathrm{t}}
$$

\subsubsection{Modelo de Confiabilidade de Software com Função de Valor Médio Tipo Gama-Generalizada}

O modelo de estatísticas de ordem gama generalizada foi proposto por Achcar, Dey e Niverthy (1996), sob a argumentação de que a classe desses modelos fornece grande flexibilidade para a forma da função intensidade $\lambda(t)$, o que implica melhores ajustes dos dados de confiabilidade de software. Os autores assumiram a função distribuição gama generalizada na modelagem da função de valor médio. Asśim, a 
função de valor médio para o modelo de estatísticas de ordem gama generalizada é dada por,

$$
\mathrm{m}(\mathrm{t})=\theta \mathrm{I}_{k}\left(\beta \mathrm{t}^{\alpha}\right)
$$

onde $\mathrm{I}_{k}$ é uma integral gama incompleta dada por,

$$
\mathrm{I}_{k}(\mathrm{~s})=\frac{1}{\Gamma(k)} \int_{0}^{\mathrm{s}} \mathrm{x}^{k-1} \mathrm{e}^{-\mathrm{x}} \mathrm{dx}
$$

e função intensidade

$$
\lambda(\mathrm{t})=\frac{\partial \mathrm{m}(\mathrm{t})}{\partial \mathrm{t}}=\frac{1}{\Gamma(k)} \theta \beta^{k} \alpha \mathrm{t}^{\alpha \mathrm{t}-1} \mathrm{e}^{-\beta \mathrm{t}^{\alpha}},
$$

onde $\theta>0, k>0, \beta>0$ e $\alpha>0$ e $\Gamma(k)$ denota a função gama.

O modelo de estatísticas de ordem gama generalizada é chamado de "super-modelo", pois incorpora outros modelos de confiabilidade de software:

(i) $\operatorname{Se} \alpha=1$ e $k=1$, a função intensidade do modelo de estatísticas de ordem gama generalizada (4.1.10) reduz-se à função intensidade do modelo de Goel e Okumoto (1979) descrita em (4.1.5).

(ii) Se $k=1,(4.1 .10)$ se reduz a (4.1.7) (Modelo de Goel, 1983).

(iii) Se $\alpha=1,(4.1 .10)$ se reduz a (4.1.8) (Modelo de estatísticas de ordem gama).

(iv) Se $k \rightarrow \infty$, temos um modelo de estatísticas de ordem normal (ver Lawless, 1982). 


\subsubsection{Modelo de Confiabilidade de Software com Função de Valor Médio do Tipo Log-Normal}

O modelo de estatísticas de ordem log-normal foi também proposto por Achcar, Dey e Niverthy (1996), considerando a distribuição log-normal na modelagem da função de valor médio. Este modelo é formulado, sob a argumentação de que a distribuição log-normal fornece grande flexibilidade para a forma da função intensidade de falha, o que implica melhores ajustes dos dados de falhas.

Considerando a função distribuição log-normal na função de valor médio (4.1.4), temos

$$
\mathrm{m}(\mathrm{t})=\theta \Phi\left(\frac{\ln t-\mu}{\sigma^{2}}\right)
$$

onde $\Phi(\cdot)$ é uma função distribuição de uma variável aleatória com distribuição normal padronizada $\mathrm{N}(0,1)$.

Derivando (4.1.12), obtemos a função intensidade de falha dada por

$$
\lambda(t)=\frac{\theta}{t \sqrt{2 \pi \sigma}} \exp \left\{-\frac{(\ln t-\mu)^{2}}{2 \sigma^{2}}\right\}
$$

onde $\theta>0,-\infty<\mu<\infty$ e $\sigma>0$.

\subsubsection{Modelo de Confiabilidade de Software com Função de Valor Médio Tipo Gaussiana Inversa}

Nesta seção, discutimos as motivações para o uso da distribuição gaussiana inversa em confiabilidade de software e apresentamos a função densidade de probabilidade e função distribuição dessa distribuição. 


\subsubsection{Motivação para a Utilização da Distribuição Gaussiana Inversa em Confiabilidade de Software}

Nos estudos de confiabilidade de software, a escolha da distribuição para modelar os dados de falhas geralmente é baseada no conhecimento a respeito do comportamento (mecanismo) de falha.

Por exemplo, é lógico considerar distribuição com taxa de falha crescente, para representar os tempos de falhas em situações onde novos erros são introduzidos no software.

Entretanto, a falha do software pode ser devida a várias causas, tais como: uso impróprio do software pelo usuário, execução de um erro de um programa, vírus, e assim por diante (ver Musa, Iannino e Okumoto, 1987).

Assim, para fazer a escolha de um modelo de falha, é mais apropriado considerar características físicas do fenômeno de falhas, do que um bom ajuste dos dados por uma distribuição (ver Chikkara e Folks, 1989).

Lawless (1982) também enfatiza esse ponto na seleção de um modelo e sugere que seja feita uma análise minuciosa de sua robustez, além de um exame cuidadoso de suposição e dados.

Quando as ocorrências de falhas antecipadas (precoces) ou correções de erros identificados no software são dominantes na distribuição dos tempos de falhas, espera-se que a taxa de falha seja uma função não monótona; primeiro crescente e depois decrescente.

Nessa situação, a distribuição gaussiana inversa (ver Chikkara e Folks, 1989) pode ser uma escolha adequada para modelar os dados de falhas do software, pois a taxa de falha dessa distribuição é uma função nãomonótona.

Embora a distribuição log-normal, entre outras, também seja aplicável em tais casos, há certas vantagens da distribuição gaussiana inversa sobre a log normal, que destacamos:

- A distribuição gaussiana inversa destina-se a uma extensa classe de distribuições dos dados de falhas, devido à grande flexibilidade da sua taxa de falha (ver figura, 4.3). 
- A taxa de falha $\lambda(\mathrm{t})$ da distribuição gaussiana inversa é diferente de zero e constante, quando $\mathrm{t} \rightarrow \infty$, mas, na distribuição log-normal, $\lambda(\mathrm{t}) \rightarrow 0$, quando $t \rightarrow \infty$.

A taxa de falha próxima de uma constante após um certo período de tempo implica que a ocorrência de falhas é aleatória e independente dos tempos de falhas anteriores. Essa é uma propriedade da distribuição exponencial, que tem sido extensivamente utilizada nos estudos de confiabilidade (ver Lawless, 1982). Por outro lado, a taxa de falha próxima de zero implica que "quase" nenhuma falha ocorrerá, o que é pouco provável na vida real.

Folks e Chikkara (1978) ajustaram a distribuição gaussiana inversa para uma variedade de conjuntos de dados referentes a estudos de confiabilidade e a distribuição gaussiana inversa, mostrou-se ser tão apropriada (adequada) quanto as distribuições Weibul e log-normal, ajustadas anteriormente por Gacula e Kubala (1975).

Na discussão do artigo de Folks e Chikkara (1978), Aitkin também ajustou duas outras distribuições de três parâmetros, a gama generalizada e a normal transformada, para os mesmos dados considerados por Folks e Chikkara (1978), mostrando que a distribuição gaussiana inversa "compete" bem com as distribuições de três parâmetros.

\subsubsection{Taxa de Falha}

A distribuição Gaussiana Inversa destina-se a uma extensa classe de distribuições dos tempos de falha, devido à grande flexibilidade de sua intensidade de falha. (ver figura 4.3). 


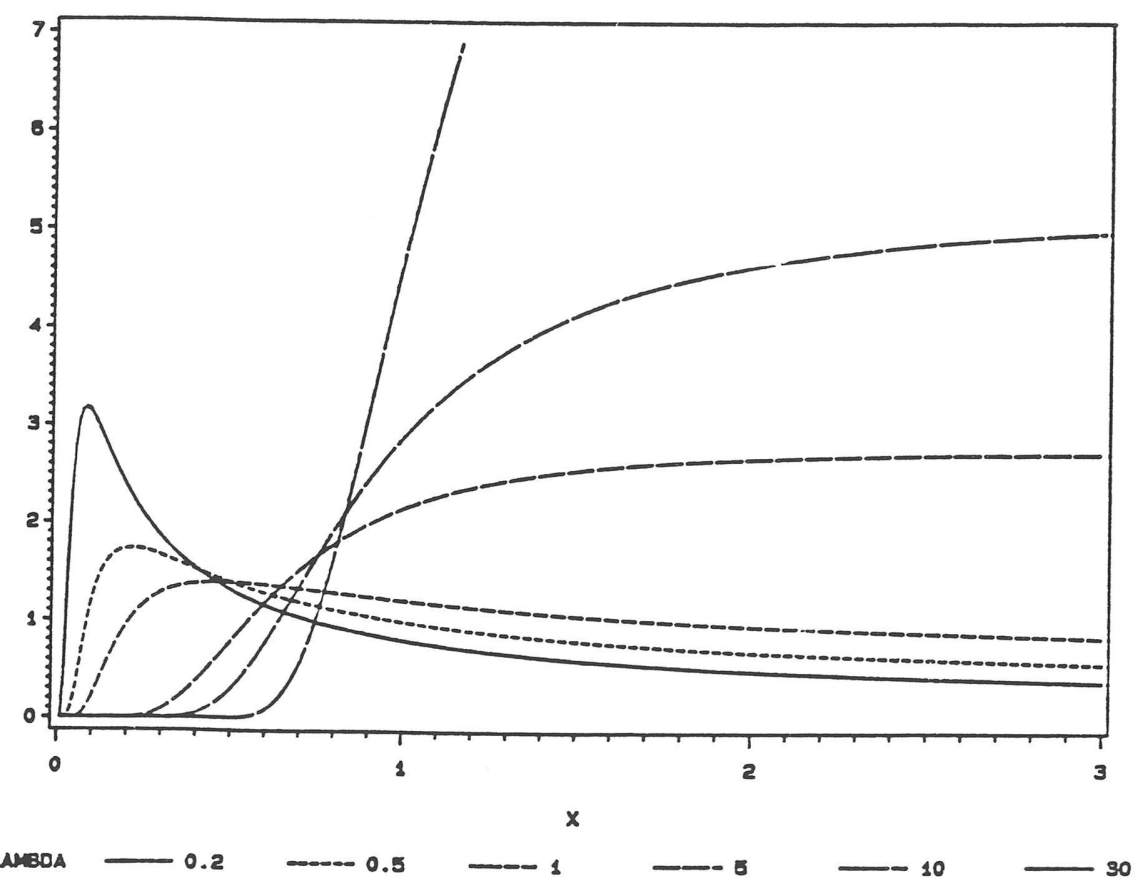

Figura 4.3. Taxa de Falha da Gaussiana Inversa com $\mu=1$ para seis valores de $\lambda$.

Através da figura (4.3), podemos observar que a taxa de falha é crescente, quando não imposta à inclinação. Assim, a distribuição gaussiana inversa também pode ser aplicada à descrição dos tempos de falhas, quando não é dominada por falhas ou correções de erros "precoces".

\subsubsection{Função Densidade de Probabilidade e Função Distribuição}

A função densidade de probabilidade da distribuição gaussiana inversa é dada por:

$$
\mathrm{f}(\mathrm{t}, \mu, \lambda)=\sqrt{\frac{\lambda}{2 \pi}} \mathrm{t}^{-3 / 2} \exp \left(-\frac{\lambda(\mathrm{t}-\mu)^{2}}{2 \mu^{2} \mathrm{t}}\right), \text { para } \mathrm{t}>0,
$$

onde $\mu>0$ e $\lambda>0$. 
A função distribuição $F(t)$ é dada por

$$
\mathrm{F}(\mathrm{t})=\Phi\left[\sqrt{\frac{\lambda}{\mathrm{t}}}\left(\frac{\mathrm{t}}{\mu}-1\right)\right]+\mathrm{e}^{2 \lambda / \mu} \Phi\left[-\sqrt{\frac{\lambda}{\mathrm{t}}}\left(1+\frac{\mathrm{t}}{\mu}\right)\right],
$$

onde $\Phi(\cdot)$ denota a função distribuição de uma variável $Z$ com distribuição normal padronizada $\mathrm{N}(0,1)$.

\subsubsection{Função de Valor Médio e Taxa de Falha Tipo Gaussiana Inversa dos PPNH-I}

Considerando a função distribuição (4.1.13), na modelagem da função de valor médio (4.1.4) temos,

$$
\mathrm{m}(\mathrm{t})=\theta\left\{\Phi\left[\sqrt{\frac{\lambda}{\mathrm{t}}}\left(\frac{\mathrm{t}}{\mu}-1\right)\right]+\mathrm{e}^{2 \lambda / \mu} \Phi\left[-\sqrt{\frac{\lambda}{\mathrm{t}}}\left(1+\frac{\mathrm{t}}{\mu}\right)\right]\right\}
$$

Derivando (4.1.14) em relação a $t$, obtemos a função intensidade $\lambda(t)$, dada por

$$
\lambda(t)=\frac{\theta \sqrt{\lambda}}{\sqrt{2 \pi}} \mathrm{t}^{-3 / 2} \exp \left\{-\frac{\lambda(\mathrm{t}-\mu)^{2}}{2 \mu^{2} \mathrm{t}}\right\} .
$$

4.2 Inferência Bayesiana para os Modelos de Confiabilidade de Software Com Processos de Poisson não Homogêneos

Como comentamos no capítulo 1 , os modelos de confiabilidade de software com processos de Poisson não homogêneos são modelos nãolineares, bastante complexos e, geralmente, apresentam vários parâmetros $\beta=\left\{\beta_{1}, \beta_{2}, \ldots, \beta_{n}\right\}$. 
O procedimento clássico usual para a análise desses modelos, consiste, inicialmente, na utilização de métodos iterativos para a obtenção das estimativas dos parâmetros (estimativas de máxima verossimilhança ou de mínimos quadrados). Porém, as distribuições dos estimadores obtidas dessa forma somente são conhecidas assintoticamente. Desse modo, inferências baseadas nessas estatísticas podem não ser confiáveis, uma vez que, em confiabilidade de software, trabalha-se com amostras não muito grandes e usualmente censuradas (ver Musa e Okumoto, 1987).

Nesta dissertação, nosso objetivo é analisar os modelos de confiabilidade de software com processos de Poisson não homogêneos sob o enfoque Bayesiano.

\subsubsection{Análise Bayesiana do Modelo de Estatísticas de Ordem Gama-Generalizada (Achcar, Dey e Niverthy, 1996)}

Nesta seção, fazemos inferências para os parâmetros do modelo de estatísticas de ordem gama generalizada, sob o enfoque Bayesiano. Para representar o grau de informação dos parâmetros, consideramos densidades a priori informativas e não informativas. Exploramos o uso de algoritmos computacionais, como o amostrador de Gibbs e Metropolis-Hastings, para aproximar as integrais a posteriori Bayesianas de interesse.

\subsubsection{Função de Verossimilhança}

Para a análise Bayesiana do modelo de estatísticas de ordem gama generalizada, consideramos o modelo de tempo truncado, testado até o tempo $\mathrm{t}_{\mathrm{n}}$. Quando terminamos o teste do software, após observar $\mathrm{n}$ falhas, substituímos $\mathrm{t}_{\mathrm{n}}$ por $\mathrm{x}_{\mathrm{n}}$ em (4.1.1) e encontramos uma expressão similar ao modelo de tempo truncado. 
Considerando-se o modelo de estatísticas de ordem gama generalizada (4.1.10), a função de verossimilhança (4.1.1), para $\theta, \alpha, \beta$ e $k$, é dada por

$$
\begin{aligned}
& \mathrm{L}\left(\theta, \alpha, \beta, k / \mathrm{D}_{\mathrm{t}}\right)=\left\{\prod_{\mathrm{i}=1}^{\mathrm{n}} \theta \frac{\beta^{k}}{\Gamma(k)} \alpha \mathrm{x}_{\mathrm{i}}^{\alpha \mathrm{k}-1} \mathrm{e}^{-\beta \mathrm{x}_{\mathrm{i}}^{\alpha}}\right\} \\
& \exp \left\{-\int_{0}^{\mathrm{t}} \theta \frac{\beta^{k}}{k} \alpha \mathrm{u}^{\alpha k-1} \mathrm{e}^{-\beta \mathrm{u}^{\alpha}} \mathrm{du} .\right\},
\end{aligned}
$$

onde $\mathrm{D}_{\mathrm{t}}=\left\{\mathrm{n}, \mathrm{x}_{1}, \mathrm{x}_{2}, \ldots, \mathrm{x}_{\mathrm{n}}\right\}$ é o conjunto de dados (ver Cox e Lewis, 1966; ou Lawless, 1982).

Portanto,

$$
\begin{aligned}
& \mathrm{L}(\theta, \alpha, \beta, k)=\frac{\theta^{\mathrm{n}} \alpha^{\mathrm{n}} \beta^{k \mathrm{n}}}{\{\Gamma(k)\}^{\mu}}\left\{\prod_{\mathrm{i}=1}^{\mathrm{n}} \mathrm{x}_{\mathrm{i}}^{\alpha k-1}\right\} \\
& \exp \left\{-\beta \sum_{\mathrm{i}=1}^{\mathrm{n}} \mathrm{x}_{\mathrm{i}}^{\alpha}-\theta \mathrm{I}_{k}\left(\beta \mathrm{t}^{\alpha}\right)\right\} .
\end{aligned}
$$

\subsubsection{Densidades a Priori para os Parâmetros $\theta, \alpha, \beta$ e $k$}

A informação de estudos preliminares ou de um especialista na área de confiabilidade de software pode ser utilizada como uma forma para melhorar os resultados inferenciais, isto é, utilizando informação a priori.

Assumindo-se o modelo de estatísticas de ordem gama generalizada, para modelar os instantes de falhas observados no intervalo $(0, t]$, vamos considerar densidades a priori informativas para expressar o grau de conhecimento sobre os parâmetros $\theta$ e $\beta$ e vamos assumir que não temos nenhuma informação prévia a respeito dos parâmetros $\alpha$ e $k$. Assim, consideramos densidades a priori não-informativas localmente uniformes, para $\alpha$ e $k$ proporcional a $\alpha^{-1}$ e $k^{-1}$ resnectivamente (ver Box e Tian 1073) 
Assumimos, então, as seguintes densidades a priori :

(i) $\theta \sim \Gamma(\mathrm{a}, \mathrm{b})$, onde a e b são constantes conhecidas e $\theta>0$;

(ii) $\beta \sim \Gamma(c, d)$, onde c e d são constantes conhecidas e $\beta>0$; (4.2.2)

(iii) $\alpha \sim \pi_{1}(\alpha)$, onde $\pi_{1}(\alpha)$ é uma densidade a priori para $\alpha(\alpha>0)$;

(iv) $k \sim \pi_{2}(k)$, onde $\pi_{2}(k)$ é uma densidade a priori para $k(k>0)$, onde $\Gamma(a, b)$ denota a distribuição gama com média $a / b$ e variância $a / b^{2}$.

\subsubsection{Densidade $a$ Posteriori Conjunta para $\theta, \alpha, \beta$ e $k$}

Assumindo independência entre os parâmetros $\theta, \alpha, \beta$ e $k$ e combinando a função de verossimilhança (4.2.1) com as densidades a priori (4.2.2) obtemos a densidade a posteriori conjunta para $\theta, \alpha, \beta$ e $k$ dada por

$$
\begin{aligned}
& \pi\left(\alpha, \beta, \theta, k \backslash D_{t}\right) \propto \frac{\theta^{\mathrm{a}+\mathrm{n}-1} \alpha^{\mathrm{n}} \beta^{k \mathrm{n}+\mathrm{c}-1}}{\{\Gamma(k)\}^{\mathrm{n}}}\left\{\prod_{\mathrm{i}=1}^{\mathrm{n}} \mathrm{x}_{\mathrm{i}}^{\alpha k-1}\right\} \\
& \exp \left\{-\left(\mathrm{d}+\sum_{\mathrm{i}=1}^{\mathrm{n}} \mathrm{x}_{\mathrm{i}}^{\alpha}\right) \beta-\left(\mathrm{b}+\mathrm{I}_{k}\left(\beta \mathrm{t}^{\alpha}\right) \theta\right\} \pi_{1}(\alpha) \pi_{2}(k),\right.
\end{aligned}
$$

onde $\alpha>0, \beta>0, \theta>0$ e $k>0$. 


\subsubsection{Densidades Condicionais de $\theta, \alpha, \beta$ e $k$ para o} Algoritmo Amostrador de Gibbs

A densidade condicional de $\theta$, para o algoritmo amostrador de Gibbs, condicionada aos dados e aos parâmetros $\alpha, \beta$ e $k$ é dada por

$$
\pi\left(\theta \backslash \alpha, \beta, k, \mathrm{D}_{\mathrm{t}}\right) \sim \Gamma\left[\mathrm{a}+\mathrm{n}, \mathrm{b}+\mathrm{I}_{k}\left(\beta \mathrm{t}^{\alpha}\right)\right],
$$

onde $\theta>0$, a e b são constantes conhecidas e $\mathrm{I}_{k}(\mathrm{~s})=\frac{1}{\Gamma(k)} \int_{0}^{\mathrm{s}} \mathrm{x}^{k-1} \mathrm{e}^{-\mathrm{x}} \mathrm{dx}$.

Note-se que (4.2.4) tem a forma da distribuição gama. Como comentamos no capítulo 3 , seção 3.3 , quando a densidade condicional tem forma padronizada, utilizamos o algoritmo amostrador de Gibbs para gerar essa variável.

A densidade condicional de $\beta$ para o algoritmo amostrador de Gibbs condicionada aos dados e aos parâmetros $\theta, \alpha$ e $k$ é dada por

$$
\pi\left(\beta \backslash \alpha, k, \theta, D_{t}\right) \propto \beta^{k n+c-1} \exp \left\{-\beta\left(\sum_{i=1}^{n} x_{i}^{\alpha}+d\right)-\theta I_{k}\left(\beta t^{\alpha}\right)\right\}(4.2 .5)
$$

onde $\beta>0$, c e d são constantes conhecidas.

Observe que a densidade condicional de $\beta$ (4.2.5) não tem forma padronizada como normal, gama, etc. Portanto, para gerar $\beta$, utilizamos o algoritmo Metropolis-Hastings.

Como comentamos no capítulo 3, nestes casos é necessário definir um núcleo de transição da cadeia de Markov do qual vamos gerar novos candidatos para o parâmetro. 
Por exemplo, se a densidade condicional $\pi(\mathrm{t})$ pode ser escrita como $\pi(\mathrm{t}) \propto \mathrm{h}(\mathrm{t}) \Psi(\mathrm{t})$, onde $\mathrm{h}(\mathrm{t})$ é uma densidade com forma padronizada (por exemplo, a densidade a priori informativa de $\beta$ ), e $\Psi(t)$ é uma função uniformemente limitada, usamos $\mathrm{h}(\mathrm{t})$ como núcleo para gerar candidatos de $\beta$.

Assim, para gerar $\beta$ pelo algoritmo de Metropolis-Hastings, primeiramente tentamos identificar um núcleo $h(t)$ na densidade condicional de $\beta$ (4.2.5). Neste caso, o núcleo identificado é uma distribuição gama $(\mathrm{c}, \mathrm{d})$. Portanto a densidade condicional de $\beta$ pode ser reescrita na forma

$$
\pi\left(\beta \backslash \alpha, k, \theta, D_{t}\right) \propto \beta^{c-1} \exp \{-\beta d\} \Psi_{3}(\alpha, \beta, \theta, k),
$$

onde $h(t) \propto \beta^{c-1} \exp \{-\beta d\}$ e

$$
\Psi_{3}(\alpha, \beta, \theta, k)=\exp \left\{-\beta \sum_{\mathrm{i}=1}^{\mathrm{n}} \mathrm{x}_{\mathrm{i}}^{\alpha}-\theta \mathrm{I}_{k}\left(\beta \mathrm{t}^{\alpha}\right)+\operatorname{kn} \log (\beta)\right\} \text {. }
$$

Se a condição de reversibilidade da cadeia de Markov é satisfeita, geramos um candidato $\beta^{(s)}$ de $\beta$, de uma distribuição gama (c,d), obtido na s-ésima simulação, com probabilidade de movimento $\left(\beta^{(s-1)}\right.$ mover-se para $\beta^{(s)}$ dada por

$$
\operatorname{mínimo}\left\{\frac{\Psi\left(\beta^{(\mathrm{s})}, \theta^{(\mathrm{s})}, \alpha^{(\mathrm{s}-1)}, k^{(\mathrm{s}-1)}\right)}{\Psi\left(\beta^{(\mathrm{s}-1)}, \theta^{(\mathrm{s})}, \alpha^{(\mathrm{s}-1)}, k^{(\mathrm{s}-1)}\right)}, 1\right\}
$$

$$
\Psi(\alpha, \beta, \theta, k)=\exp \left\{-\beta \sum_{\mathrm{i}=1}^{\mathrm{n}} \mathrm{x}_{\mathrm{i}}^{\alpha}-\theta \mathrm{I}_{k}\left(\beta \mathrm{t}^{\alpha}\right)+\mathrm{kn} \log (\beta)\right\} .
$$

Caso contrário, permanecemos com $\beta^{(s-1)}$. 
A densidade condicional de $\alpha$ para o algoritmo amostrador de Gibbs é dada por

$$
\begin{aligned}
& \pi\left(\alpha \backslash \theta, \beta, k, D_{t}\right) \propto \alpha^{n}\left\{\prod_{i=1}^{n} x_{i}^{\alpha k-1}\right\} \exp \left\{-\beta \sum_{i=1}^{n} x_{i}^{\alpha}-\theta I_{k}\left(\beta t^{\alpha}\right)\right\} \\
& \times \pi_{1}(\alpha)
\end{aligned}
$$

onde $\pi_{1}(\alpha) \propto \frac{1}{\alpha}$ (densidade a priori não-informativa localmente uniforme (ver Box e Tiao, 1973)) para $\alpha>0$.

Observe-se que a densidade condicional de $\alpha$ (4.2.6) não tem forma conhecida e também não pode ser escrita na forma, $\pi(t) \propto h(t) \Psi(t)$, pois não é identificado nenhum núcleo $(\mathrm{h}(\mathrm{t}))$ em (4.2.6).

Quando isso ocorre, multiplicamos e dividimos a densidade condicional de $\alpha$ (4.2.6) por uma função densidade com forma conhecida e simétrica (Chib e Greenberg, 1995) e a consideramos como a distribuição para gerar candidatos de $\alpha$.

Neste caso, a distribuição que gera candidatos de $\alpha$ é a gama $(e, f)$, (para $\alpha>0$ ).

$\mathrm{Na}$ prática, os valores $e, \mathrm{f}$ podem ser determinados a partir de informações como a média e a variabilidade do parâmetro de interesse através de estudos dos dados de falhas.

Caso seja satisfeita a reversibilidade da cadeia de Markov, gera-se um candidato $\alpha^{(\mathrm{s})}$ (da s-ésima iteração) de $\alpha$, pela distribuição gama $(e, \mathrm{f})$ com probabilidade de movimento,

$$
\operatorname{minimo}\left\{\frac{\Psi_{1}\left(\alpha^{(\mathrm{s})}, \beta^{(\mathrm{s})}, \theta^{(\mathrm{s})}, k^{(\mathrm{s}-1)}\right)}{\Psi_{1}\left(\alpha^{(\mathrm{s}-1)}, \beta^{(\mathrm{s})}, \theta^{(\mathrm{s})}, k^{(\mathrm{s}-1)}\right)}, 1\right\} \text {, }
$$


onde

$$
\begin{aligned}
& \Psi_{1}(\alpha, \beta, \theta, k)=\exp \left\{-\beta \sum_{i=1}^{n} x_{i}^{\alpha}-\theta I_{k}\left(\beta t^{\alpha}\right)-n \log (\alpha)+\right\} \\
& \times \exp \left\{(\alpha k-1) \sum_{i=1}^{n} \log \left(x_{i}\right)-f \alpha-(e-2) \log (\alpha)\right\} .
\end{aligned}
$$

Caso contrário, permanecemos com $\alpha^{(\mathrm{s}-1)}$.

A densidade condicional de $k$ para o algoritmo amostrador de Gibbs é dada por,

$$
\begin{aligned}
& \pi\left(k \backslash \alpha, \beta, \theta, D_{t}\right) \propto \frac{\beta^{k n}}{\{\Gamma(k)\}^{n}}\left\{\prod_{i=1}^{n} x_{i}^{\alpha k-1}\right\} \\
& \exp \left\{\theta I_{k}\left(\beta t^{\alpha}\right)\right\} \pi_{2}(k)
\end{aligned}
$$

onde $\mathrm{k}>0$ e $\pi_{2}(k) \propto \frac{1}{k}$ (densidade a priori localmente uniforme (ver Box e Tiao, 1973).

A densidade condicional de $k$ (4.2.7) não tem forma conhecida e não pode ser escrita como $\pi(\mathrm{t}) \propto \mathrm{h}(\mathrm{t}) \Psi(\mathrm{t})$, pois não se identifica nenhum núcleo $(\mathrm{h}(\mathrm{t}))$ em (4.2.7).

Portanto, multiplicamos e dividimos (4.2.7) por uma distribuição gama $(\mathrm{g}, \mathrm{h})$ e por essa mesma distribuição, gera-se $k$ com probabilidade de movimento dada por,

$$
\operatorname{minimo}\left\{\frac{\Psi_{2}\left(\alpha^{(\mathrm{s})}, \beta^{(\mathrm{s})}, \theta^{(\mathrm{s})}, k^{(\mathrm{s})}\right)}{\Psi_{2}\left(\alpha^{(\mathrm{s})}, \beta^{(\mathrm{s})}, \theta^{(\mathrm{s})}, k^{(\mathrm{s}-1)}\right)}, 1\right\}
$$


onde

$$
\begin{aligned}
& \Psi_{2}(\alpha, \beta, \theta, k)=\exp \{k \mathrm{n} \log (\beta)-\mathrm{n} \log (\Gamma(k))\} \\
& \times \exp \left\{\sum_{\mathrm{i}=1}^{\mathrm{n}} \log \left(\mathrm{x}_{\mathrm{i}}^{\alpha k-1}\right)+\theta \mathrm{I}_{k}\left(\beta \mathrm{t}^{\alpha}\right)-\log (k)-\mathrm{h} k+\mathrm{g} \log (k)\right\} .
\end{aligned}
$$

Note-se que, para gerar as variáveis $\alpha, \beta$ e $k$, é necessária a utilização do algoritmo de Metropolis-Hastings (ver por exemplo, Chib e Greenberg, 1995), e para gerar $\theta$, a distribuição gama $\Gamma\left[\mathrm{a}+\mathrm{n}, \mathrm{b}+\mathrm{I}_{k}\left(\beta \mathrm{t}^{\alpha}\right)\right]$ envolve o cálculo de $\mathrm{I}_{k}\left(\beta \mathrm{t}^{\alpha}\right)$, em cada iteração.

\subsubsection{Análise Bayesiana do Modelo de Estatísticas de Ordem Gama Generalizada (Achcar, Dey e Niverthy, 1996) Considerando a Inclusão da Variável Latente $\left(\mathrm{N}^{\prime}=\mathrm{N}-\mathrm{n}\right)$}

Nesta seção, fazemos inferências sobre os parâmetros do modelo de estatísticas de ordem gama-generalizada, considerando as densidades $a$ priori para $\theta, \alpha, \beta$ e $k$, definidas na seção (4.2.1.2).

Para aproximar as integrais a posteriori de interesse de $\theta, \alpha, \beta$ e $k$, utilizamos a técnica de dados aumentados (ver Tanner e Young, 1987) para o amostrador de Gibbs. Este aumento dos dados facilita a especificação das densidades condicionais dos parâmetros desconhecidos, dada uma variável latente. Assim, consideramos a inclusão da variável latente $\mathrm{N}^{\prime}=\mathrm{N}-\mathrm{n}$ (ver Yang, 1994, Kuo e Yang, 1996 ou Achcar, Dey e Niverthy, 1996) onde $N^{\prime}$ é o número de erros remanescentes no software. A inclusão da variável $\mathrm{N}^{\prime}$ (onde $\mathrm{N}^{\prime}$ é um número restrito positivo e inteiro) com distribuição $a$ priori Poisson com parâmetro $\theta(1-\mathrm{F}(\mathrm{t}))$, denotada por, $\mathrm{N}^{\prime} \sim$ $\mathrm{P}[\theta(1-\mathrm{F}(\mathrm{t}))]$, simplifica as expressões das densidades condicionais, pois a função distribuição $\mathrm{F}(\mathrm{t})$, que envolve o cálculo da integral gama incompleta, 
é excluída das densidades condicionais dos parâmetros de interesse $\theta, \alpha, \beta$ e $k$.

\subsubsection{Função de Verossimilhança}

Considerando-se o modelo de estatísticas de ordem gama generalizada (4.1.14), a função de verossimilhança (4.1.1), para $\theta, \alpha, \beta$ e $k$, é dada por

$$
\begin{aligned}
& \mathrm{L}\left(\theta, \alpha, \beta, k \backslash \mathrm{D}_{\mathrm{t}}\right)=\left\{\prod_{\mathrm{i}=1}^{\mathrm{n}} \theta \frac{\beta^{k}}{\Gamma(k)} \alpha \mathrm{x}_{\mathrm{i}}^{\alpha \mathrm{k}-1} \mathrm{e}^{-\beta \mathrm{x}_{\mathrm{i}}^{\alpha}}\right\} \\
& \exp \left\{-\int_{0}^{\mathrm{t}} \theta \frac{\beta^{k}}{k} \alpha \mathrm{u}^{\alpha k-1} \mathrm{e}^{-\beta \mathrm{u}^{\alpha}} d \mathrm{u} .\right\} .
\end{aligned}
$$

Portanto,

$$
\begin{aligned}
& \mathrm{L}(\theta, \alpha, \beta, k)=\frac{\theta^{\mathrm{n}} \alpha^{\mathrm{n}} \beta^{k \mathrm{n}}}{\{\Gamma(k)\}}\left\{\prod_{\mathrm{i}=1}^{\mathrm{n}} \mathrm{x}_{\mathrm{i}}^{\alpha k-1}\right\} \\
& \exp \left\{-\beta \sum_{\mathrm{i}=1}^{\mathrm{n}} \mathrm{x}_{\mathrm{i}}^{\alpha}-\theta \mathrm{I}_{k}\left(\beta \mathrm{t}^{\alpha}\right)\right\}
\end{aligned}
$$

\subsubsection{Densidades a Priori para os Parâmetros} $\mathrm{N}^{\prime}, \theta, \alpha, \beta$ e $k$

Para expressarmos o grau de conhecimento sobre $\mathrm{N}^{\prime}, \theta, \alpha, \beta$ e $k$ assumimos as seguintes densidades a priori:

(i) $\mathrm{N}^{\prime} \sim \mathrm{P}\left\{\theta\left[1-\mathrm{I}_{\mathrm{k}}\left(\beta \mathrm{t}^{\alpha}\right)\right\}\right.$, para $\mathrm{N}^{\prime}>0$; 
(ii) $\theta \sim \Gamma(\mathrm{a}, \mathrm{b})$, onde a e b são conhecidos e $\theta>0$;

(iii) $\beta \sim \Gamma(\mathrm{c}, \mathrm{d})$, onde c e d são conhecidos e $\beta>0$;

(iv) $\alpha \sim \pi_{1}(\alpha)$, onde $\pi_{1}(\alpha)$ é uma densidade a priori $\operatorname{para} \alpha(\alpha>0)$;

(v) $k \sim \pi_{2}(k)$, onde $\pi_{2}(k)$ é uma densidade a priori para $k(k>0)$.

As densidades a priori para os parâmetros $\theta, \alpha, \beta$ e $k$ são definidas em (4.2.2) e $\mathrm{P}(\lambda)$ denota a distribuição de Poisson com parâmetro $\lambda$; $\Gamma(\mathrm{a}, \mathrm{b})$ denota a distribuição gama com média $\mathrm{a} / \mathrm{b}$ e variância $\mathrm{a} / \mathrm{b}^{2}$.

Assumindo que o pesquisador não tenha nenhuma informação prévia a respeito dos parâmetros $\alpha$ e $k$, vamos considerar densidades a priori não-informativas e localmente uniformes para $\alpha$ e $k$, proporcional a $\alpha^{-1} \mathrm{e}$ $k^{-1}$, respectivamente (ver Box e Tiao, 1973).

\subsubsection{Densidade a Posteriori Conjunta para $\mathrm{N}^{\prime}, \theta, \alpha, \beta$ e $k$}

Assumindo independência entre os parâmetros $\theta, \alpha, \beta$ e $k$ e combinando a função de verossimilhança (4.2.8) com as densidades a priori (4.2.9), obtemos a densidade a posteriori conjunta para os parâmetros $\mathrm{N}^{\prime}, \theta, \alpha, \beta$ e $k$, dada por:

$$
\begin{aligned}
& \pi\left(\mathrm{N}^{\prime}, \alpha, \beta, k, \theta \backslash \mathrm{D}_{\mathrm{t}}\right) \propto \frac{\theta^{\mathrm{N}^{\prime}+\mathrm{n}+\alpha-1} \alpha^{\mathrm{n}} \beta^{k \mathrm{n}+\mathrm{c}-1}}{\mathrm{~N}^{\prime} !\left\{\Gamma(k)^{\mathrm{n}}\right\}} \\
& \mathrm{x}\left\{\prod_{\mathrm{i}=1}^{\mathrm{n}} \mathrm{x}_{\mathrm{i}}^{\alpha k-1}\right\}\left\{1-\mathrm{I}_{k}\left(\beta \mathrm{t}^{\alpha}\right)\right\}^{\mathrm{N}^{\prime}} \mathrm{e}^{-(\mathrm{b}+1) \theta-\left(\mathrm{d}+\sum_{\mathrm{i}=1}^{\mathrm{n}} \mathrm{x}_{\mathrm{i}}^{\alpha}\right) \beta} \pi_{1}(\alpha) \pi_{2}(\beta),
\end{aligned}
$$


onde $\mathrm{N}^{\prime}>0, \alpha>0, \beta>0, \theta>0$ e $k>0$.

\subsubsection{Densidades Condicionais para $\mathrm{N}^{\prime}, \theta, \alpha, \beta$ e $k$} para o Algoritmo de Gibbs

(i) $\mathrm{N}^{\prime} \backslash \alpha, \beta, k, \theta, \mathrm{D}_{\mathrm{t}} \sim \mathrm{P}\left[\theta\left(1-\mathrm{I}_{k}\left(\beta \mathrm{t}^{\alpha}\right)\right)\right]$;

(ii) $\theta \backslash \mathrm{N}^{\prime}, \alpha, \beta, k, \mathrm{D}_{\mathrm{t}} \sim \Gamma\left[\mathrm{a}+\mathrm{n}+\mathrm{N}^{\prime}, \mathrm{b}+1\right]$;

(iii) $\pi\left(\beta \backslash \mathrm{N}^{\prime}, \alpha, k, \theta, \mathrm{D}_{\mathrm{t}}\right) \propto \beta^{k \mathrm{n}+\mathrm{c}-1} \mathrm{e}^{-\beta\left[\sum_{\mathrm{i}=1}^{\mathrm{n} x_{\mathrm{i}}^{\alpha}+\mathrm{d}}\right]}\left\{1-\mathrm{I}_{\mathrm{k}}\left(\beta \mathrm{t}^{\alpha}\right)\right\}^{\mathrm{N}^{\prime}}$;

(iv) $\pi\left(\alpha \backslash \theta, N^{\prime}, \beta, k, D_{t}\right) \propto \alpha^{n}\left\{\prod_{i=1}^{n} x_{i}^{\alpha k-1}\right\} e^{-\beta \sum_{i=1}^{n} x_{i}^{\alpha}}$ $\left\{1-\mathrm{I}_{k}\left(\beta \mathrm{t}^{\alpha}\right)\right\}^{\mathrm{N}^{\prime}} \pi_{1}(\alpha)$

(v) $\pi\left(k \backslash N^{\prime}, \alpha, \beta, \theta, D_{\mathrm{t}}\right) \propto \frac{\beta^{k \mathrm{n}}}{\{\Gamma(k)\}^{\mathrm{n}}}\left\{\prod_{\mathrm{i}=1}^{\mathrm{n}} \mathrm{x}_{\mathrm{i}}^{\alpha k-1}\right\}$

$\left\{1-\mathrm{I}_{k}\left(\beta \mathrm{t}^{\alpha}\right)\right\}^{\mathrm{N}^{\prime}} \pi_{2}(k)$.

As variáveis $\alpha, \beta$ e $k$ são geradas pelo algoritmo de Metropolis Hastings.

Observe-se que a introdução da variável latente $\mathrm{N}^{\prime}=\mathrm{N}-\mathrm{n}$, na análise Bayesiana desse modelo, proporciona expressões mais simples das densidades condicionais; diminuindo a complexidade das expressões (densidades condicionais), diminui o tempo computacional para a convergência dos algoritmos de Metropolis Hastings e amostrador de Gibbs. 


\subsubsection{Análise Bayesiana do Modelo de Goel Considerando a Inclusão da Variável Latente $\left(\mathrm{N}^{\prime}=\mathrm{N}-\mathrm{n}\right)$}

(1983)

Assumindo $\mathrm{k}=1$ no modelo de estatísticas de ordem gama generalizada com função de valor (4.1.19), obtemos o modelo de estatísticas de ordem Weibull (processo de Goel, 1983).

\subsubsection{Densidade a Posteriori Conjunta}

Assumindo independência entre os parâmetros $\mathrm{N}^{\prime}, \alpha, \beta$ e $\theta$ e $\mathrm{k}=1$, na função de verossimilhança do modelo de estatísticas de ordem gama generalizada (4.2.1), obtemos a função de verossimilhança do modelo de Goel (1983). Então, combinamos essa função com as densidades a priori para $\mathrm{N}^{\prime}, \alpha, \beta$ e $\theta$ definidas em (4.2.9); assim, temos a densidade a posteriori conjunta dada por

$$
\begin{aligned}
& \pi\left(N^{\prime}, \alpha, \beta, \theta \backslash D_{t}\right) \propto \frac{1}{N^{\prime} !} \theta^{N^{\prime}+n+a-1} \alpha^{n} \beta^{n+c-1} \\
& x\left\{\prod_{i=1}^{n} x_{i}^{\alpha-1}\right\} e^{-(b+1) \theta} e^{-\left(\sum_{i=1}^{n} x_{i}^{\alpha}+N^{\prime} t^{\alpha}+d\right) \beta} \pi_{1}(\alpha),
\end{aligned}
$$

onde $\mathrm{N}^{\prime}>0, \alpha>0, \beta>0$ e $\theta>0$.

\subsubsection{Densidades Condicionais para $\mathrm{N}^{\prime}, \alpha, \beta$ e $\theta$ para o Algoritmo de Gibbs}

As densidades condicionais de $\mathrm{N}^{\prime}, \alpha, \beta$ e $\theta$, para o algoritmo amostrador de Gibbs são dadas por, 
(i) $\mathrm{N}^{\prime} \backslash \alpha, \beta, \theta, \mathrm{D}_{\mathrm{t}} \sim \mathrm{P}\left[\theta\left(1-\mathrm{I}\left(\beta \mathrm{t}^{\alpha}\right)\right)\right]$;

(ii) $\theta \backslash N^{\prime}, \alpha, \beta, D_{t} \sim \Gamma\left[a+n+N^{\prime}, b+1\right]$;

(iii) $\pi\left(\beta \backslash N^{\prime}, \alpha, \theta, D_{t}\right) \sim \Gamma\left[n+c, N^{\prime} t^{c}+d+\sum_{i=1}^{n} x_{i}^{\alpha}\right]$;

(iv) $\pi\left(\alpha \backslash \theta, \mathrm{N}^{\prime}, \beta, k, \mathrm{D}_{\mathrm{t}}\right) \propto \alpha^{\mathrm{n}}\left\{\prod_{\mathrm{i}=1}^{\mathrm{n}} \mathrm{x}_{\mathrm{i}}^{\alpha}\right\} \mathrm{e}^{-\left(\mathrm{N}^{\prime} \mathrm{t}+\sum_{\mathrm{i}=1}^{\mathrm{n}} \mathrm{x}_{\mathrm{i}}^{\alpha}\right) \beta} \pi_{1}(\alpha)$.

Atente-se que, quando k=1 (modelo de Goel, 1983), utilizamos o algoritmo de Metrópolis-Hastings para gerar somente a variável $\alpha$.

\subsubsection{Análise Bayesiana para o Modelo de Estatísticas de Ordem Gama Considerando a Inclusão da Variável Latente $\left(\mathbf{N}^{\prime}=\mathbf{N}-\mathbf{n}\right)$}

Admitindo-se $\alpha=1$ no modelo de estatísticas de ordem gama generalizada temos o modelo de estatísticas de ordem gama.

\subsubsection{Densidade a Posteriori Conjunta de $\mathrm{N}^{\prime}, \beta, k$ e $\theta$}

Assumindo independência para os parâmetros $\mathrm{N}^{\prime}, \beta, k$ e $\theta$, a densidade a posteriori conjunta para $\mathrm{N}^{\prime}, \beta, k$ e $\theta$ é obtida assumindo $\alpha=1$, na função de verossimilhança (4.2.8), e combinando essa função com as densidades a priori de $\mathrm{N}^{\prime}, \beta, k$ e $\theta$ definidas em (4.2.9). Assim, temos, 


$$
\begin{aligned}
& \pi\left(\mathrm{N}^{\prime}, \beta, \theta, k \backslash \mathrm{D}_{\mathrm{t}}\right) \propto \frac{1}{\mathrm{~N}^{\prime}\{\Gamma(k)\}^{\mathrm{n}}} \theta^{\mathrm{N}^{\prime}+\mathrm{n}+\mathrm{a}-1} \beta^{k \mathrm{n}+\mathrm{c}-1} \\
& \left\{\prod_{\mathrm{i}=1}^{\mathrm{n}} \mathrm{x}_{\mathrm{i}}^{\mathrm{k}-1}\right\} \mathrm{e}^{-(\mathrm{b}+1) \theta} \mathrm{e}^{-\left(\mathrm{d}+\sum_{\mathrm{i}=1}^{\mathrm{n}} \mathrm{x}_{\mathrm{i}}\right) \beta}\left\{1-\mathrm{I}_{k}(\beta \mathrm{t})\right\}^{\mathrm{N}^{\prime}} \pi_{2}(k),
\end{aligned}
$$

onde $\mathrm{N}^{\prime}>0, \beta>0, \theta>0$ e $k>0$.

\subsubsection{Densidades Condicionais para $\mathrm{N}^{\prime}, \beta, k$ e $\theta$ para o algoritmo de Gibbs}

As densidades condicionais de $\mathrm{N}^{\prime}, \beta, k$ e $\theta$ para o algoritmo amostrador de Gibbs são dadas por:

(i) $\mathrm{N}^{\prime} \backslash \beta, k, \theta, \mathrm{D}_{\mathrm{t}} \sim \mathrm{P}\left[\theta\left(1-\mathrm{I}_{k}(\beta \mathrm{t})\right)\right]$;

(ii) $\theta \backslash \mathrm{N}^{\prime}, \beta, k, \mathrm{D}_{\mathrm{t}} \sim \Gamma\left[\mathrm{a}+\mathrm{n}+\mathrm{N}^{\prime}, \mathrm{b}+1\right]$;

(iii) $\pi\left(\beta \backslash \mathrm{N}^{\prime}, k, \theta, \mathrm{D}_{\mathrm{t}}\right) \propto \beta^{k \mathrm{n}+\mathrm{c}-1} \mathrm{e}^{-\beta\left[\sum_{\mathrm{i}=1}^{\mathrm{n}} \mathrm{x}_{\mathrm{i}}+\mathrm{d}\right]}\left\{1-\mathrm{I}_{\mathrm{k}}(\beta \mathrm{t})\right\}^{\mathrm{N}^{\prime}}$;

(iv) $\pi\left(k \backslash \mathrm{N}^{\prime}, \beta, \theta, \mathrm{D}_{\mathrm{t}}\right) \propto \frac{\beta^{k \mathrm{n}}}{\{\Gamma(k)\}^{\mathrm{n}}}\left\{\prod_{\mathrm{i}=1}^{\mathrm{n}} \mathrm{x}_{\mathrm{i}}^{k-1}\right\}\left\{1-\mathrm{I}_{k}(\beta \mathrm{t})\right\}^{\mathrm{N}^{\prime}} \pi_{2}(k)$.

Atente-se que, quando $\alpha=1$, torna-se necessária a utilização do algoritmo de Metrópolis-Hastings para gerar as variáveis $\beta$ e $k$. 


\subsubsection{Análise Bayesiana para o Modelo de Goel e Okumoto (1979) Considerando a Inclusão da Variável Latente $\left(\mathbf{N}^{\prime}=\mathbf{N}-\mathbf{n}\right)$}

Quando $\alpha=1$ e $k=1$ no modelo de estatísticas de ordem gama generalizada, obtemos o modelo de estatísticas de ordem exponencial (processo de Goel e Okumoto, 1979).

\subsubsection{Densidade a Posteriori Conjunta de $\mathrm{N}^{\prime}, \theta$ e $\beta$}

Assumindo independência entre os parâmetros $\mathrm{N}^{\prime}, \theta$ e $\beta$ e combinando as densidades a priori de $\mathrm{N}^{\prime}, \theta$ e $\beta$, definidas em (4.2.9) com a função de verossimilhança (4.2.8) com $\alpha=1$ e $k=1$, encontramos a densidade a posteriori conjunta para $\mathrm{N}^{\prime}, \theta$ e $\beta$ dada por,

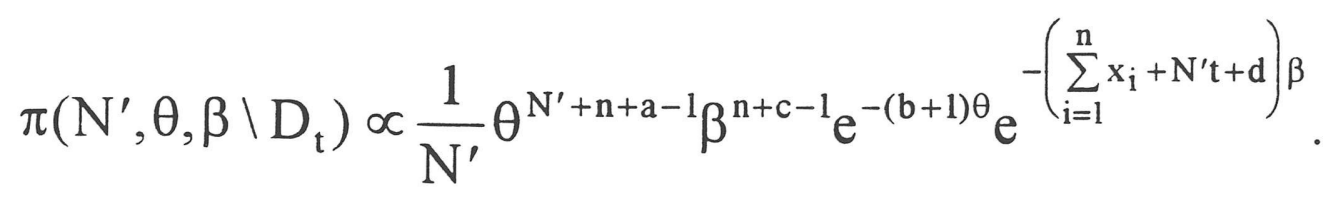

onde $\mathrm{N}^{\prime}>0, \beta>0$ e $\theta>0$.

4.2.5.2 Densidades condicionais para $\mathrm{N}^{\prime}, \theta$ e $\beta$ para 0 algoritmo de Gibbs

As densidades condicionais de $\mathrm{N}^{\prime}, \theta$ e $\beta$ para o algoritmo amostrador de Gibbs são dadas por: 
(i) $\mathrm{N}^{\prime} \backslash \beta, \theta, \mathrm{D}_{\mathrm{t}} \sim \mathrm{P}\left[\theta \mathrm{e}^{-\beta \mathrm{t}^{\alpha}}\right]$;

(ii) $\theta \backslash \mathrm{N}^{\prime}, \beta, \mathrm{D}_{\mathrm{t}} \sim \Gamma\left[\mathrm{a}+\mathrm{n}+\mathrm{N}^{\prime}, \mathrm{b}+1\right]$;

(ii) $\pi\left(\beta \backslash \mathrm{N}^{\prime}, \theta, \mathrm{D}_{\mathrm{t}}\right) \sim \Gamma\left[\mathrm{n}+\mathrm{c}, \mathrm{N}^{\prime} \mathrm{t}+\mathrm{d}+\sum_{\mathrm{i}=1}^{\mathrm{n}} \mathrm{x}_{\mathrm{i}}\right]$.

\subsubsection{Análise Bayesiana do Modelo de Estatísticas de Ordem log-normal (Achcar, Dey e Niverthy Considerando a Inclusão da Variável Latente $\left(\mathrm{N}^{\prime}=\mathrm{N}-\mathrm{n}\right)$ \\ (1996))}

Nesta seção, apresentamos uma análise Bayesiana do modelo de estatísticas de ordem log-normal (ver Achcar, Dey e Niverthy, 1996). Consideramos a técnica de dados aumentados com inclusão de uma variável latente $\mathrm{N}^{\prime}=\mathrm{N}-\mathrm{n}$, para facilitar a especificação das densidades condicionais do algoritmo amostrador de Gibbs e Metropolis-Hastings.

\subsubsection{Função de Verossimilhança}

Tomando-se o modelo de falha truncado, a função de verossimilhança do modelo de estatísticas de ordem log-normal, com função de valor médio (4.1.12), é dada por

$$
\begin{aligned}
& \mathrm{L}_{\lg }\left(\theta, \mu, \sigma \backslash \mathrm{D}_{\mathrm{t}}\right)=\frac{\theta^{\mathrm{n}}}{(2 \pi)^{\mathrm{n} / 2} \sigma^{\mathrm{n}}}\left\{\prod_{\mathrm{i}=1}^{\mathrm{n}} 1 / \mathrm{x}_{\mathrm{i}}\right\} \\
& \exp \left\{-\frac{1}{2 \sigma^{2}} \sum_{\mathrm{i}=1}^{\mathrm{n}}\left(\ln \mathrm{x}_{\mathrm{i}}-\mu\right)^{2}-\theta \Phi\left(\frac{\ln \mathrm{t}-\mu}{\sigma}\right)\right\} .
\end{aligned}
$$




\subsubsection{Densidades a Priori para $N^{\prime}, \theta, \mu$ e $\sigma$}

Supondo que o pesquisador não tenha informação prévia a respeito dos parâmetros $\mu \mathrm{e} \sigma$, vamos considerar densidades a priori não informativas e localmente uniformes para $\mu$ e $\sigma$ (ver Box e Tiao, 1973) e vamos assumir densidades a priori informativas para os parâmetros $\mathrm{N}^{\prime}$ e $\theta$.

Assim, assumimos para $\mathrm{N}^{\prime}, \theta, \mu \mathrm{e} \sigma$ as seguintes densidades a priori,

(i) $\mathrm{N}^{\prime} \sim \mathrm{P}\left[\theta\left(1-\Phi\left(\frac{\ln \mathrm{t}-\mu}{\sigma}\right)\right)\right]$, para $\mathrm{N}^{\prime}>0$;

(ii) $\theta \sim \Gamma\left[a_{1}, b_{1}\right]$, onde $a_{1}$ e $b_{1}$ são conhecidos e $\theta>0$;

(iii) $\mu \sim \pi_{3}(\mu)$, onde $\pi_{3}(\mu)$ é a densidade a priori para $\mu(-\infty<\mu<\infty)$;

(iv) $\sigma \sim \pi_{4}(\sigma)$, onde $\pi_{4}(\sigma)$ é a densidade a priori para $\sigma(\sigma>0)$.

\subsubsection{Densidade a Posteriori Conjunta para} $\mathrm{N}^{\prime}, \theta, \mu$ e $\sigma$

Combinando as densidades a priori (4.2.16) e a função de verossimilhança (4.2.15), encontramos a densidade a posteriori conjunta para $\mathrm{N}^{\prime}, \theta, \mu$ e $\sigma$, dada por, 


$$
\begin{aligned}
& \pi\left(\mathrm{N}^{\prime}, \theta, \mu, \sigma \backslash \mathrm{D}_{\mathrm{t}}\right) \propto \frac{1}{\sigma^{\mathrm{n}} \mathrm{N}^{\prime} !} \theta^{\mathrm{n}+\mathrm{N}^{\prime}+\mathrm{a}-1} \mathrm{e}^{-\theta(\mathrm{b}+1)} \\
& \times\left\{1-\Phi\left(\frac{\ln \mathrm{t}-\mu}{\sigma}\right)\right\}^{\mathrm{N}^{\prime}} \exp \left\{-\frac{1}{2 \sigma^{2}} \sum_{\mathrm{i}=1}^{\mathrm{n}}\left(\ln \mathrm{x}_{\mathrm{i}}-\mu\right)^{2}\right\} \pi_{3}(\mu) \pi_{4}(\sigma) .
\end{aligned}
$$

4.2.6.4 Densidades Condicionais para $\mathrm{N}^{\prime}, \theta, \mu$ e $\sigma$ para

\section{o Algoritmo Amostrador de Gibbs}

As densidades condicionais de $\mathrm{N}^{\prime}, \theta, \mu$ e $\sigma$ para o algoritmo amostrador de Gibbs são dadas por:

$$
\begin{aligned}
& \text { (i) } \mathrm{N}^{\prime} \backslash \theta, \mu, \sigma, \mathrm{D}_{\mathrm{t}} \sim \mathrm{P}\left[\theta\left(1-\Phi\left(\frac{\ln \mathrm{t}-\mu}{\sigma}\right)\right)\right] \\
& \text { (ii) } \theta \backslash \mathrm{N}^{\prime}, \mu, \sigma, \mathrm{D}_{\mathrm{t}} \sim \Gamma\left[\mathrm{a}+\mathrm{n}+\mathrm{N}^{\prime}, \mathrm{b}+1\right] \\
& \text { (iii) } \mu \backslash \mathrm{N}^{\prime}, \theta, \sigma, \mathrm{D}_{\mathrm{t}} \propto \exp \left\{-\frac{1}{2 \sigma^{2}} \sum_{\mathrm{i}=1}^{\mathrm{n}}\left(\ln \mathrm{x}_{\mathrm{i}}-\mu\right)^{2}\right\} \\
& \times\left\{1-\Phi\left(\frac{\ln \mathrm{t}-\mu}{\sigma}\right)\right\}^{\mathrm{N}^{\prime}} \pi_{3}(\mu) ; \\
& \text { (iv) } \sigma \backslash \mathrm{N}^{\prime}, \mu, \theta, \mathrm{D}_{\mathrm{t}} \propto \exp \left\{-\frac{1}{2 \sigma^{2}} \sum_{\mathrm{i}=1}^{\mathrm{n}}\left(\ln \mathrm{x}_{\mathrm{i}}-\mu\right)^{2}\right\} \\
& \times \sigma^{-\mathrm{n}}\left\{1-\Phi\left(\frac{\ln \mathrm{t}-\mu}{\sigma}\right)\right\}^{\mathrm{N}^{\prime}} \pi_{4}(\sigma) .
\end{aligned}
$$

onde $\Phi(\cdot)$ é a função distribuição de uma variável aleatória $Z \sim N(0,1)$ (Normal padronizada). 


\subsubsection{Análise Bayesiana do Modelo de Estatísticas de Ordem Gaussiana Inversa (Achcar e Storani, 1996) Considerando a Inclusão da Variável Latente $\left(N^{\prime}=N-n\right)$}

Nesta seção, fazemos inferências para os parâmetros do modelo de estatísticas de ordem gaussiana inversa sob o aspecto Bayesiano. Apresentamos as densidades condicionais do algoritmo de Gibbs com Metropolis para os parâmetros $\theta, \mu \mathrm{e} \lambda$. Utilizamos a técnica de dados aumentados para as densidades condicionais do amostrador de Gibbs, incluída a variável latente $\mathrm{N}^{\prime}=\mathrm{N}-\mathrm{n}$.

\subsubsection{Função de Verossimilhança}

Considerando-se o modelo de estatísticas de ordem gaussiana inversa com função intensidade de falha (4.1.14), a função de verossimilhança para $\theta, \mu$ e $\lambda$ é dada por,

$$
\begin{aligned}
& \mathrm{L}_{\mathrm{GI}}(\theta, \mu, \lambda)=\frac{\theta^{\mathrm{n}} \lambda^{\mathrm{n} / 2}}{(2 \pi)^{\mathrm{n} / 2}}\left\{\prod_{\mathrm{i}=1}^{\mathrm{n}} \mathrm{x}_{\mathrm{i}}^{-3 / 2}\right\} \\
& \exp \left\{-\theta \mathrm{F}\left(\mathrm{x}_{\mathrm{n}}\right)-\frac{\lambda}{2 \mu^{2}} \sum_{\mathrm{i}=1}^{\mathrm{n}} \frac{\left(\mathrm{x}_{\mathrm{i}}-\mu\right)^{2}}{\mathrm{x}_{\mathrm{i}}}\right\},
\end{aligned}
$$

onde $\mathrm{F}(\mathrm{t})=\Phi\left[\sqrt{\frac{\lambda}{\mathrm{t}}}\left(\frac{\mathrm{t}}{\mu}-1\right)\right]+\mathrm{e}^{2 \lambda / \mu} \Phi\left[-\sqrt{\frac{\lambda}{\mathrm{t}}}\left(1+\frac{\mathrm{t}}{\mu}\right)\right]$ é definida em (4.1.13) e $\Phi(\cdot)$ é a função distribuição de uma variável aleatória $Z \sim N(0,1)$ (normal padronizada). 


\subsubsection{Densidades a Priori Para os Parâmetros $\mathrm{N}^{\prime}, \theta$, $\mu$ e $\lambda$}

O grau de conhecimento sobre os parâmetros é expresso pela distribuição a priori. Supondo que temos informação sobre os parâmetros de interesse, a partir de opinião de especialistas da área de software, ou através de estudos preliminares, vamos supor densidades a priori informativas para $\mathrm{N}^{\prime}, \theta, \mu$ e $\lambda$ dadas por,

(i) $\mathrm{N}^{\prime} \sim \mathrm{P}[\theta[1-\mathrm{F}(\mathrm{t})]]$,para $\mathrm{N}^{\prime}>0$;

(ii) $\mu \sim \Gamma\left(a_{2}, b_{2}\right) ; a_{2}, b_{2}$ conhecidos, para $\mu>0$;

(iii) $\lambda \sim \Gamma\left(\mathrm{a}_{3}, \mathrm{~b}_{3}\right) ; \mathrm{a}_{3}, \mathrm{~b}_{3}$ conhecidos, para $\lambda>0$;

(iv) $\theta \sim \Gamma\left(\mathrm{a}_{4}, \mathrm{~b}_{4}\right) ; \mathrm{a}_{4}, \mathrm{~b}_{4}$ conhecidos, para $\theta>0$.

onde $\mathrm{P}(\lambda)$ denota distribuição de Poisson com parâmetro $\lambda, \Gamma\left(\mathrm{a}_{\mathrm{i}}, \mathrm{b}_{\mathrm{i}}\right)$ $(i=2,3,4)$ denota a distribuição gama com média $a_{i} / b_{i}$ e variância $a_{i} /\left(b_{i}\right)^{2}$.

\section{2.7.3 Densidade a Posteriori Conjunta de $N^{\prime}, \theta, \mu$ e $\lambda$}

Admitindo-se independência entre os parâmetros $\theta, \mu$ e $\lambda$ e combinando a função de verossimilhança (4.2.18) com as densidades $a$ priori (4.2.19), obtemos a densidade a posteriori conjunta dada por 


$$
\begin{aligned}
& \pi\left(N^{\prime}, \mu, \lambda, \theta \backslash D_{t}\right) \propto \theta^{N^{\prime}+n+a_{3}-1} \lambda^{n / 2+a_{2}-1} \mu^{a_{1}-1} \frac{\left\{1-F\left(x_{n}\right)\right\}^{N^{\prime}}}{N^{\prime} !} \\
& \exp \left\{-\frac{\lambda}{2 \mu^{2}} \sum_{i=1}^{n} \frac{\left(x_{i}-\mu\right)^{2}}{x_{i}}-b_{1} \mu-b_{2} \lambda-\theta\left(b_{3}+1\right)\right\},
\end{aligned}
$$

onde $D_{t}=\left\{n, x_{1}, \ldots, x_{n}, t\right\}$ é o conjunto de dados e $F(t)$ é dado em (4.1.13).

\subsubsection{Densidades Condicionais de $\mathrm{N}^{\prime}, \theta, \mu$ e $\lambda$ para o algoritmo amostrador de Gibbs}

As densidades condicionais de $\mathrm{N}^{\prime}, \theta, \mu$ e $\lambda$ para o algoritmo amostrador de Gibbs são dadas por,

(i) $\mathrm{N}^{\prime} \backslash \mu, \lambda, \theta, \mathrm{D} \sim \mathrm{P}\left[\theta\left(1-\mathrm{F}\left(\mathrm{x}_{\mathrm{n}}\right)\right)\right]$;

(ii) $\theta \backslash \mu, \lambda, \mathrm{N}^{\prime}, \mathrm{D} \sim \Gamma\left[\mathrm{N}^{\prime}+\mathrm{n}+\mathrm{a}_{4}, \mathrm{~b}_{4}+1\right]$;

(iii) $\pi\left(\mu \backslash \lambda, \theta, N^{\prime}, D\right) \propto \mu^{\mathrm{a}_{2}-1} \mathrm{e}^{-\mathrm{b}_{2} \mu} \Psi_{1}(\mu, \lambda)$,

onde $\Psi_{1}(\mu, \lambda)=\exp \left\{-\frac{\lambda}{2 \mu^{2}} \sum_{\mathrm{i}=1}^{\mathrm{n}} \frac{\left(\mathrm{x}_{\mathrm{i}}-\mu\right)^{2}}{\mathrm{x}_{\mathrm{i}}}+\mathrm{N}^{\prime} \log \left(1-\mathrm{F}\left(\mathrm{x}_{\mathrm{n}}\right)\right)\right\}$;

(iv) $\pi\left(\lambda \backslash \theta, \mu, N^{\prime}\right.$, D) $\propto \lambda^{a_{3}-1} e^{-b_{3} \lambda} \Psi_{2}(\mu, \lambda)$

onde $\Psi_{2}(\mu, \lambda)=\exp \left\{N^{\prime} \log \left(1-F\left(x_{n}\right)\right)+\frac{n}{2} \log (\lambda)-\frac{\lambda}{2 \mu^{2}} \sum_{i=1}^{n} \frac{\left(x_{i}-\mu\right)^{2}}{x_{i}}\right\}$.

Observe-se que utilizamos o algoritmo Metropolis-Hastings para gerar $\mu$ e $\lambda$. 


\section{Capítulo 5}

\section{DISCRIMINAÇÃO DOS MODELOS DE CONFIABILIDADE DE SOFTWARE}

Como apresentado nos capítulos 2 e 4, a literatura discute vários modelos de estratégia tipo II para análise dos dados de falhas de um software, dentre os quais destacamos os processos de Poisson homogêneos: modelo de Moranda e generalização do modelo de Moranda e os processos de Poisson não homogêneos: modelos de estatísticas de ordem gama, gamageneralizada, log-normal, exponencial, Weibul e gaussiana inversa.

Uma questão que surge é a seguinte "Dentre os modelos que utilizam processos de Poisson trata-se de saber qual (ou quais) é aquele que melhor se ajusta a um determinado conjunto de dados de confiabilidade de software?"

Para determinarmos o melhor modelo, dois fatores devem ser considerados (Gelfand, Dey e Chang, 1992): adequabilidade e seleção do modelo.

Sob o enfoque Bayesiano, procura-se, neste trabalho, responder a esta questão através dos seguintes critérios:

(i) Soma dos Erros Relativos;

(ii) Análise frequencial de densidades preditivas condicionais ordenadas, denotada por PCPO (em inglês "prequential conditional predictive ordinates") (Yang, 1994). 
A técnica PCPO define o $\mathrm{CPO}^{1}$, de $\mathrm{t}_{\mathrm{i}}$ condicionado aos dados passados dos tempos de falhas $t_{1}, \ldots, t_{i-1}$ conjuntamente. Essa técnica difere do CPO que utiliza os dados cross-validated, onde o condicionamento é de todos os dados passados e futuros exceto $t_{i}$. A análise frequencial é considerada, pois o teste do software é usualmente conduzido em um tempo real. Para cada instante de falha, temos interesse em predizer o instante da próxima falha. A regra CPO providencia uma taxa de explicação dos dados para o modelo em estudo.

Gelfand, Dey e Chang (1992) fizeram uma revisão da técnica CPO dos dados cross-validated na discriminação de modelos.

Dawid (1984) propõe, para a seleção dos modelos de confiabilidade de software, o critério da verossimilhança frequencial. Singpurwalla e Soyer (1992) também utilizam a análise frequencial para a comparação dos modelos onde a confiabilidade crescente de um software é modelada por um processo auto-regressivo de coeficiente aleatório.

Yang (1994) e Achcar, Dey e Niverthy (1996) consideram a técnica PCPO para a discriminação dos modelos de confiabilidade de software que utilizam os processos de Poisson não homogêneo.

Para a verificação da adequabilidade do modelo, Littlewood, AbdelGhaly e Chan (1986) propõem o u-plot; ordenando os valores $u_{i}=P\left(T_{i}<t_{i}\right)$ e plotando os pontos $\left(u_{j}, j / c\right)$, onde c é o número total de falhas no software, observadas no intervalo $(0, t]$. Algum vício na predição será destacado pelo desvio da linha unitária com inclinação na origem.

Portanto, além dos procedimentos analíticos de discriminação, podese também ter uma boa idéia do melhor modelo com processos de Poisson não homogêneo, através das suposições básicas dos modelos de estatísticas de ordem PPNH-I: O número de erros $\mathrm{N}$ no início do teste do software tem distribuição de Poisson com média $\theta$. Então, a função de valor médio é

\footnotetext{
${ }^{1}$ CPO é a densidade preditiva condicional ordenada; para o tempo de falha futuro $t_{i+1}$ é definido ser $c_{i+1}=p\left(t_{i+1} / t_{1}, t_{2}, . . t_{i-1}, t_{i}, t_{i+2}, \ldots, t_{n}\right)$.
} 
dada por $m(t)=\theta F(t)$. Se essas suposições estiverem corretas, então $\mathrm{m}(\mathrm{t}) / \theta=\mathrm{F}(\mathrm{t})$ tem uma distribuição uniforme padronizada.

Assim, para cada tempo de falha, podemos considerar Q-Q plots empíricos de estimadores monte Carlo de $\mathrm{m}(\mathrm{t}) / \theta$ versus uma distribuição Uniforme $(0,1)$ para cada tempo de falha (ver Achcar, Dey e Niverthy, 1996). Um distanciamento da distribuição uniforme indica inadequação do modelo.

É importante ressaltarmos que várias outras estratégias Bayesianas ou clássicas, propostas na literatura, podem ser utilizadas na discriminação dos modelos de confiabilidade de software. Além das estratégias Bayesianas de discriminação citadas anteriormente, acrescenta-se, o Fator de Bayes (Jeffreys, 1939), Pseudo Fator de Bayes (Geisser e Eddy, 1979), etc.

Neste capítulo, são apresentadas as técnicas de discriminação de modelo PCPO e soma dos erros relativos. Essas estratégias são apresentadas utilizando-se os modelos de Moranda, generalização do modelo de Moranda e os modelos de estatísticas de ordem exponencial, gama, gama generalizada, log-normal, Weibul e gaussiana inversa.

\subsection{Seleção dos Modelos que Utilizam Processos de Poisson Homogêneo}

Um critério simples para a seleção dos modelos com processos de Poisson homogêneos, modelo de Moranda e a generalização do modelo de Moranda, é o das somas dos erros de predição relativos, dado por

$$
\mathrm{SRPE}=\sum_{\mathrm{i}=1}^{\mathrm{n}} \frac{\left|\mathrm{m}_{\mathrm{i}}-\mathrm{E}\left(\mathrm{m}_{\mathrm{i}} \backslash \mathrm{m}_{1}, \ldots, \mathrm{m}_{\mathrm{i}-1}\right)\right|}{\mathrm{E}\left(\mathrm{m}_{\mathrm{i}} \backslash \mathrm{m}_{1}, \ldots, \mathrm{m}_{\mathrm{i}-1}\right)}, \text { para } \mathrm{i}=2,3 \ldots
$$


A densidade preditiva para a observação futura $\mathrm{m}_{\mathrm{i}}$, do modelo de Moranda com função intensidade (2.1.1), condicionada $\mathrm{m}_{1}, \mathrm{~m}_{2}, \ldots, \mathrm{m}_{\mathrm{i}-1}$, $(i=2,3, \ldots)$ é definida por,

$\mathrm{f}\left(\mathrm{m}_{\mathrm{i}} \backslash \mathrm{m}_{1}, \mathrm{~m}_{2}, \ldots, \mathrm{m}_{\mathrm{i}-1}\right)=\int_{0}^{1} \int_{0}^{\infty} \mathrm{f}\left(\mathrm{m}_{\mathrm{i}} \backslash \lambda, \mathrm{k}_{1}\right) \pi\left(\lambda, \mathrm{k}_{1} \backslash \mathrm{m}_{1}, \mathrm{~m}_{2}, \ldots, \mathrm{m}_{\mathrm{i}-1}\right) \mathrm{d} \lambda \mathrm{dk}_{1}$

onde $f\left(m_{i} \backslash \lambda\right)=\frac{e^{-\lambda_{i}} \lambda_{i}^{m_{i}}}{m_{i} !}, m_{i}=0,1,2, \ldots ; \lambda_{i}$ dado em (2.1.1)

Assumindo $\mathrm{k}_{1}$ conhecido em (5.1.2) e uma densidade a priori não informativa proporcional a $\lambda^{-1}$ para $\lambda$, a densidade preditiva para o número de falhas $\mathrm{m}_{1}$, condicionada aos dados $\mathrm{m}_{1}, \mathrm{~m}_{2}, \ldots, \mathrm{m}_{\mathrm{i}-1}(\mathrm{i}=2,3, \ldots)$, é dada por,

$\mathrm{f}\left(\mathrm{m}_{\mathrm{i}} / \mathrm{m}_{1}, \mathrm{~m}_{2}, \ldots, \mathrm{m}_{\mathrm{i}-1}\right)=\int_{0}^{\infty} \mathrm{f}\left(\mathrm{m}_{\mathrm{i}} / \lambda\right) \pi\left(\lambda / \mathrm{m}_{1}, \mathrm{~m}_{2}, \ldots, \mathrm{m}_{\mathrm{i}-1}\right) \mathrm{d} \lambda,(5.1 .3)$

onde $f\left(m_{i} / \lambda\right)=\frac{e^{-\lambda_{i}} \lambda_{i}^{m_{i}}}{m_{i} !}, m_{i}=0,1,2, \ldots ; \lambda_{i}$ dado em (2.1.1)

Considerando a expressão (5.1.3), temos

$f\left(m_{i} / m_{1}, m_{2}, \ldots, m_{i-1}\right) \propto \frac{k_{1}^{(i-1) m_{i}}}{m_{i} !} \int_{0}^{\infty} \lambda^{d_{1}(i)+m_{i}-1} \exp \left\{-\lambda\left[k_{1}^{i-1}+\sum_{j=1}^{i-1} k_{1}^{j-1}\right]\right\} d \lambda$

onde $d_{1}(i)=\sum_{j=1}^{i-1} m_{j}$ 
Assim, a densidade preditiva para o modelo com função intensidade (2.1.1) é dada por

$$
\mathrm{f}\left(\mathrm{m}_{\mathrm{i}} / \mathrm{m}_{1}, \mathrm{~m}_{2}, \ldots \mathrm{m}_{\mathrm{i}-1}\right)=\frac{\mathrm{c}_{1} \theta_{\mathrm{i}}^{\mathrm{m}_{\mathrm{i}}} \Gamma\left(\mathrm{d}_{1}(\mathrm{i})+\mathrm{m}_{\mathrm{i}}\right)}{\mathrm{m}_{\mathrm{i}} !}
$$

onde $\theta_{\mathrm{i}}=\frac{\mathrm{k}_{1}^{\mathrm{i}-1}}{\left[\mathrm{k}_{1}^{\mathrm{i}-1}+\sum_{\mathrm{j}=1}^{\mathrm{i}-1} \mathrm{k}_{1}^{\mathrm{j}-1}\right]}, \mathrm{d}_{1}(\mathrm{i})=\sum_{\mathrm{j}=1}^{\mathrm{i}-1} \mathrm{~m}_{\mathrm{j}}, \Gamma(\mathrm{x})$ é a função gama e $\mathrm{c}_{1}$ é a constante normalizadora dada por,

$$
\mathrm{c}_{1}^{-1}=\sum_{\mathrm{m}_{1}=0}^{\infty} \frac{\theta_{\mathrm{i}}^{\mathrm{m}_{\mathrm{i}}} \Gamma\left(\mathrm{d}_{1}(\mathrm{i})+\mathrm{m}_{\mathrm{i}}\right)}{\mathrm{m}_{\mathrm{i}} !}
$$

Observe-se que a constante normalizadora $c_{1}$, definida em (5.1.4), pode ser escrita na forma:

$$
\mathrm{c}_{1}^{-1}=\Gamma\left[\mathrm{d}_{1}(\mathrm{i})\right]\left\{1+\frac{\theta_{\mathrm{i}}}{1 !} \mathrm{d}_{1}(\mathrm{i})+\frac{\theta_{\mathrm{i}}^{2}}{2 !} \mathrm{d}_{1}(\mathrm{i})\left(\mathrm{d}_{1}(\mathrm{i})+1\right)+\ldots\right\}
$$

isto é,

$$
\mathrm{c}_{1}^{-1}=\Gamma\left[\mathrm{d}_{1}(\mathrm{i})\right] \sum_{\mathrm{j}=0}^{\infty}\left(\begin{array}{c}
\mathrm{d}_{1}(\mathrm{i})+\mathrm{j}-1 \\
\mathrm{j}
\end{array}\right) \theta_{\mathrm{i}}^{\mathrm{j}}
$$


Fazendo uma transformação de variáveis $\mathrm{x}=\mathrm{d}_{1}(\mathrm{i})+\mathrm{j}$, temos a expressão:

$$
\sum_{j=0}^{\infty}\left(\begin{array}{c}
d_{1}(i)+j-1 \\
j
\end{array}\right) \theta_{i}^{j}=\sum_{x=d_{1}(i)}^{\infty}\left(\begin{array}{l}
x-1 \\
x-d_{1}(i)
\end{array}\right) \theta_{i}^{x-d_{1}(i)}
$$

(ver por exemplo, Abramowitz e Stegun, 1972).

Assim, a constante normalizadora de (5.1.3) é escrita como,

$$
\mathrm{c}_{1}^{-1}=\Gamma\left[\mathrm{d}_{1}(\mathrm{i})\right]\left(1-\theta_{\mathrm{i}}\right)^{-\mathrm{d}_{1}(\mathrm{i})} .
$$

Substituindo-se (5.1.5) em (5.1.4), a densidade preditiva para o número de falhas é dada por

$$
\mathrm{f}\left(\mathrm{m}_{\mathrm{i}} / \mathrm{m}_{1}, \mathrm{~m}_{2}, \ldots, \mathrm{m}_{\mathrm{i}-1}\right)=\frac{\theta_{\mathrm{i}}^{\mathrm{m}_{\mathrm{i}}}\left(1-\theta_{\mathrm{i}}\right)^{\mathrm{d}_{1}(\mathrm{i})} \Gamma\left(\mathrm{d}_{1}(\mathrm{i})+\mathrm{m}_{\mathrm{i}}\right)}{\mathrm{m}_{\mathrm{i}} ! \Gamma\left[\mathrm{d}_{1}(\mathrm{i})\right]}
$$

onde $\mathrm{m}_{\mathrm{i}}=0,1,2, .$. e $\mathrm{d}_{1}(\mathrm{i})=\sum_{\mathrm{j}=1}^{\mathrm{i}-1} \mathrm{~m}_{\mathrm{j}}$

Considerando-se o modelo de Moranda (1975), o valor médio de predição para $\mathrm{m}_{\mathrm{i}}$ condicionado a $\mathrm{m}_{1}, \mathrm{~m}_{2}, \ldots, \mathrm{m}_{\mathrm{i}-1}(\mathrm{i}=2,3, \ldots)$ é definido por:

$$
E\left(m_{i} / m_{1}, m_{2}, \ldots, m_{i-1}\right)=\sum_{m_{i}=0}^{\infty} m_{i} f\left(m_{i} / m_{1}, m_{2}, \ldots, m_{i-1}\right)
$$


Considerando-se a densidade preditiva (5.1.6), o valor médio de predição (5.1.7) é dado por:

$$
E\left(m_{i} / m_{1}, m_{2}, \ldots, m_{i-1}\right)=\frac{k_{1}^{i} \sum_{j=1}^{i-1} m_{j}}{\sum_{j=1}^{i-1} k_{1}^{j}} .
$$

(ver Achcar, 1995).

Para a generalização do modelo de Moranda (Achcar, 1995), a densidade preditiva de uma observação futura $\mathrm{m}_{\mathrm{i}}$, dado $\mathrm{m}_{1}, \mathrm{~m}_{2}, \ldots, \mathrm{m}_{\mathrm{i}-1}$, $(i=2,3, \ldots)$, é definida por

$$
\begin{array}{r}
\mathrm{f}\left(\mathrm{m}_{\mathrm{i}} / \mathrm{m}_{1}, \mathrm{~m}_{2}, \ldots, \mathrm{m}_{\mathrm{i}-1}\right)=\int_{-\infty}^{\infty} \int_{0}^{1} \int_{0}^{\infty} \mathrm{f}\left(\mathrm{m}_{\mathrm{i}} / \lambda_{\mathrm{a}}, \mathrm{k}_{1}, \mathrm{k}_{2}\right) \\
\times \pi\left(\lambda_{\mathrm{a}}, \mathrm{k}_{1}, \mathrm{k}_{2} / \mathrm{m}_{1}, \mathrm{~m}_{2}, \ldots, \mathrm{m}_{\mathrm{i}-1}\right) \mathrm{d} \lambda_{\mathrm{a}} \mathrm{dk}_{1} \mathrm{dk}_{2}
\end{array}
$$

onde $\mathrm{f}\left(\mathrm{m}_{\mathrm{i}} / \lambda_{\mathrm{a}}\right)=\frac{\mathrm{e}^{-\lambda_{\mathrm{i}}} \lambda_{\mathrm{i}}^{\mathrm{m}_{\mathrm{i}}}}{\mathrm{m}_{\mathrm{i}} !}, \mathrm{m}_{\mathrm{i}}=0,1,2, \ldots ; \lambda_{\mathrm{i}}$ dado em $(2.2 .1)$

Considerando-se $\mathrm{k}_{1}$ e $\mathrm{k}_{2}$ conhecidos em (5.1.9), e seguindo-se o mesmo procedimento de cálculo da densidade preditiva do Modelo de Moranda (5.1.6), a densidade preditiva para o número de falhas $\mathrm{m}_{\mathrm{i}}$ condicionada a $\mathrm{m}_{1}, \mathrm{~m}_{2}, \ldots, \mathrm{m}_{\mathrm{i}-1},(\mathrm{i}=2,3, \ldots)$, é dada por:

$$
f\left(m_{i} / m_{1}, m_{2}, \ldots, m_{i-1}\right)=\frac{\theta_{i}^{m_{i}}\left(1-\theta_{i}\right)^{d_{1}(i)} \Gamma\left(d_{1}(i)+m_{i}\right)}{m_{i} ! \Gamma\left[d_{1}(i)\right]}
$$


onde $\mathrm{m}_{\mathrm{i}}=0,1,2, \ldots \quad \theta_{\mathrm{i}}=\frac{\mathrm{k}_{1}^{\mathrm{i}^{\mathrm{k}_{2}}}}{\left[\mathrm{k}_{1}^{\mathrm{i}^{\mathrm{k}_{2}}} \sum_{\mathrm{j}=1}^{\mathrm{i}-1} \mathrm{k}_{1}^{\mathrm{j}^{\mathrm{k}_{2}}}\right]} \quad \mathrm{e} \quad \mathrm{d}_{1}(\mathrm{i})=\sum_{\mathrm{j}=1}^{\mathrm{i}-1} \mathrm{~m}_{\mathrm{j}}$.

O valor médio de predição para a generalização do modelo de Moranda é dado por:

$$
E\left(m_{i} / m_{1}, m_{2}, \ldots, m_{i-1}\right)=\frac{k_{1}^{\mathrm{i}^{k}} \sum_{j=1}^{i-1} m_{j}}{\sum_{j=1}^{i-1} k_{1}^{j^{k_{2}}}}
$$

Segundo esse critério, o modelo selecionado é aquele que minimiza a soma dos erros de predição médio (5.1.1).

Para a seleção dos modelos, podemos também considerar a densidade preditiva ordenada frequencial definida por $\mathrm{c}_{\mathrm{i}}=\mathrm{f}\left(\mathrm{m}_{\mathrm{i}} \backslash \mathrm{m}_{1}, \ldots, \mathrm{m}_{\mathrm{i}-1}\right)$.

As densidades preditivas (5.1.3) e (5.1.9) podem ser aproximadas por estimadores Monte Carlo, considerando-se as amostras geradas pelo amostrador de Gibbs com Metropolis.

Um estimador Monte Carlo de (5.1.9), considerando-se as amostras geradas pelo amostrador de Gibbs é dado por

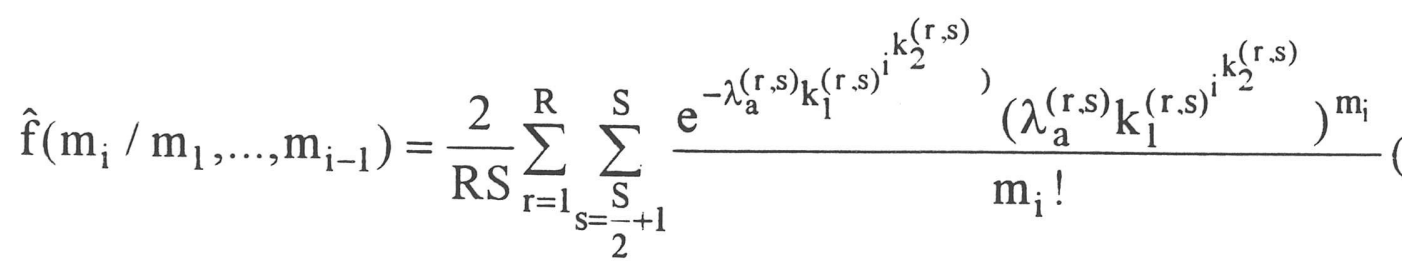

De maneira análoga, aproximamos a densidade preditiva do modelo de Moranda (5.1.2), por um estimador Monte Carlo, utilizando as amostras 
geradas pelo amostrador de Gibbs e Metropolis-Hastings. A densidade preditiva (5.1.2) aproximada é definida em (3.4.5).

Podemos "plotar" $c_{i}=\hat{f}\left(m_{i} \backslash m_{1}, \ldots, m_{i-1}\right)$ versus $i$, para $i=2, . ., n$, para cada modelo. $\mathrm{O}$ modelo que apresentar, em média, valores maiores de $\mathrm{c}_{i}$, é o melhor. O critério da verossimilhança preditiva Bayesiano, seleciona o modelo que maximiza a verossimilhança $\mathrm{c}(\ell)=\prod_{\mathrm{i}=1}^{\mathrm{n}} \mathrm{c}_{\mathrm{i}}(\ell)$, onde $\ell$ indexa $\mathrm{o} \ell$ ésimo modelo de confiabilidade. Neste caso, $(\ell=1,2), \quad(\ell=1$ modelo de Moranda, $€=2$ generalização do modelo de Moranda).

\subsection{Seleção dos Modelos de Confiabilidade de Software que utilizam os Processos de Poisson não homogêneos}

Para os modelos PPNH-I, podemos considerar a técnica da verossimilhança preditiva condicional ordenada, denotada por PCPO. A técnica PCPO define o CPO de $t_{i}$ condicionado aos dados passados dos tempos de falhas $t_{1}, \ldots, t_{i-1}$ conjuntamente.

A análise frequencial é considerada, porque o teste do software é usualmente conduzido em um tempo real.

Nesta seção os dados são definidos ser os instantes de falhas do software, denotados por $\mathrm{x}_{1}, \ldots, \mathrm{x}_{\mathrm{n}}$. O conjunto de dados é escrito por $D_{x_{i}}=\left\{x_{1}, \ldots, x_{n}\right\}$.

Admitindo-se os modelos de estatísticas de ordem denotados por PPNH-I, a verossimilhança da densidade preditiva condicional ordenada para uma observação futura $x_{i+1}$ é definida por $c_{i}=p\left(x_{i+1} / D_{x_{i}}\right)$, ou seja, é a densidade condicional estimada de $\mathrm{X}_{\mathrm{i}+1}$ para o tempo futuro observado de falha $x_{i+1}$, condicionado aos dados $\left\{x_{1}, \ldots, x_{i}\right\}$.

A seqüência $\left\{X_{i}\right\}_{i \geq 1}$ é uma cadeia de Markov (ver Yang, 1994, pág. 52). Portanto, $c_{i}=p\left(x_{i+1} \backslash D_{x_{i}}\right)$ pode ser escrito como $c_{i}=p\left(x_{i+1} \backslash x_{i}\right)$. 
Assim, a densidade preditiva condicional ordenada pode ser calculada por

$$
\begin{aligned}
& p\left(x_{i+1} \backslash x_{i}\right)=\int p\left(x_{i+1} \backslash \beta, D_{x_{i}}\right) p\left(\beta, D_{x_{i}}\right) d \beta \\
= & \int \lambda_{I I}\left(x_{i+1}\right) \exp \left\{-m_{I I}\left(x_{i+1}\right)+m_{I I}\left(x_{i}\right)\right\} p\left(\beta, D_{x_{i}}\right) d \beta
\end{aligned}
$$

onde $\mathrm{m}_{\mathrm{II}}(\mathrm{t})=-\ln (1-\mathrm{F}(\mathrm{t}))$ é a função de valor médio dos PPNH-II, definida em (1.2.4) e $\lambda_{\Pi}(\mathrm{t})$ é a função densidade dada por $\lambda_{\text {II }}(\mathrm{t})=\frac{\mathrm{dm}_{\mathrm{II}}(\mathrm{t})}{\mathrm{dt}}=\frac{\mathrm{f}(\mathrm{t})}{(1-\mathrm{F}(\mathrm{t}))}$.

Considerando-se o modelo de estatísticas de ordem gama generalizada (4.1.10) com $\mathrm{F}(\mathrm{t})=\mathrm{I}_{k}\left(-\beta \mathrm{t}^{\alpha}\right)$, temos $\mathrm{m}_{\mathrm{I}}(\mathrm{t})=-\ln \left(1-\mathrm{I}_{k}\left(\beta \mathrm{t}^{\alpha}\right)\right)$ e $\lambda_{\text {II }}(\mathrm{t})=\alpha \beta^{k} \mathrm{t}^{\alpha k-1} \mathrm{e}^{-\beta \mathrm{t}^{\alpha}} /\left\{\Gamma(k)\left[1-\mathrm{I}_{k}\left(\beta \mathrm{t}^{\alpha}\right)\right]\right\}$.

Para o modelo de estatísticas de ordem gama generalizada, o PCPO (5.2.1) é dado por

$$
\mathrm{p}\left(\mathrm{x}_{\mathrm{i}+1} \backslash \mathrm{x}_{\mathrm{i}}\right)=\iiint \frac{\alpha \beta^{k} \mathrm{x}_{\mathrm{i}+1}^{\alpha k-1} \mathrm{e}^{-\beta \mathrm{x}_{\mathrm{i}+1}^{\alpha}}}{\Gamma(k)\left\{1-\mathrm{I}_{k}\left(\beta \mathrm{x}_{\mathrm{i}}^{\alpha}\right)\right\}} \mathrm{p}\left(\alpha, \beta, k \backslash \mathrm{D}_{\mathrm{x}_{\mathrm{i}}}\right) \mathrm{d} \alpha \mathrm{d} \beta \mathrm{d} k .
$$

Utilizando-se o amostrador de Gibbs e Metropolis-Hastings, (5.2.2) pode ser aproximado por um estimador Monte Carlo dado por

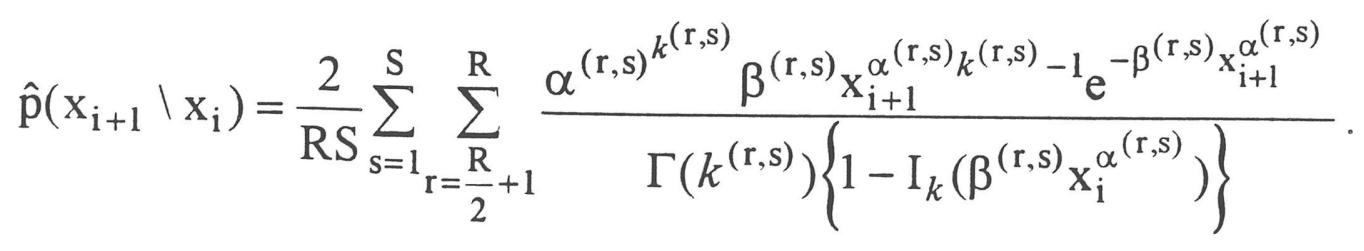


O PCPO dos modelos de estatísticas de ordem exponencial, gama e Weibul são facilmente obtidos de (5.2.2); por exemplo, considerando-se k=1 em (5.2.2), temos o PCPO dos modelos de estatísticas de ordem Weibul dado por,

$$
\mathrm{p}\left(\mathrm{x}_{\mathrm{i}+1} \backslash \mathrm{x}_{\mathrm{i}}\right)=\iint \alpha \beta \mathrm{x}_{\mathrm{i}+1}^{\alpha-1} \exp \left\{-\beta \mathrm{x}_{\mathrm{i}+1}^{\alpha}+\beta \mathrm{x}_{\mathrm{i}}^{\alpha}\right\} \mathrm{p}\left(\alpha, \beta \backslash \mathrm{D}_{\mathrm{x}_{\mathrm{i}}}\right) \mathrm{d} \alpha \mathrm{d} \beta .
$$

O PCPO (5.2.3), pode ser aproximado por um estimador Monte Carlo, utilizando-se as amostras geradas pelo amostrador de Gibbs e Metropolis-Hastings.

Considere-se, agora, o modelo de estatísticas de ordem log-normal com função de valor médio (4.1.12), a função de valor médio dos PPNH-II (1.2.4) é dada por $\mathrm{m}_{\mathrm{II}}(\mathrm{t})=-\ln \left\{1-\Phi\left(\frac{\ln \mathrm{t}-\mu}{\sigma}\right)\right\}$ e a função intensidade $\lambda_{\text {II }}(t)=m_{I I}^{\prime}(t)=\frac{\exp \left\{-(\ln t-\mu)^{2} / 2 \sigma^{2}\right\}}{\sqrt{2 \pi} \sigma t\left\{1-\Phi\left(\frac{\ln t-\mu}{\sigma}\right)\right\}}$.

O PCPO (5.2.1), para o instante de falha futura $x_{i+1}$, dos modelos de estatísticas de ordem log-normal, é dado por,

$$
\mathrm{p}\left(\mathrm{x}_{\mathrm{i}+1} \backslash \mathrm{x}_{\mathrm{i}}\right)=\iint \frac{\exp \left\{-\ln \left(\mathrm{x}_{\mathrm{i}+1}-\mu\right)^{2} / 2 \sigma^{2}\right\}}{\sqrt{2 \pi} \sigma \mathrm{x}_{\mathrm{i}+1}\left\{1-\Phi\left(\frac{\ln \mathrm{x}_{\mathrm{i}+1}-\mu}{\sigma}\right)\right\}} \mathrm{p}\left(\mu, \sigma \backslash \mathrm{D}_{\mathrm{x}_{\mathrm{i}}}\right) \mathrm{d} \mu \mathrm{d} \sigma .(5.2 .4)
$$

Admitindo-se o modelo de estatísticas de ordem gaussiana inversa com função distribuição (4.1.13), a função de valor médio (1.2.4) é dada por $\mathrm{m}_{\text {II }}(\mathrm{t})=-\ln \left\{1-\left[\Phi\left[\sqrt{\frac{\lambda}{\mathrm{t}}}\left(\frac{\mathrm{t}}{\mu}-1\right)\right]+\mathrm{e}^{2 \lambda / \mu} \Phi\left[-\sqrt{\frac{\lambda}{\mathrm{t}}}\left(1+\frac{\mathrm{t}}{\mu}\right)\right]\right]\right\}$ e a função 
intensidade $\lambda_{\mathrm{II}}(\mathrm{t})=\frac{\frac{\sqrt{\lambda}}{\sqrt{2 \pi}} \mathrm{t}^{-3 / 2} \exp \left\{-\frac{\lambda(\mathrm{t}-\mu)^{2}}{2 \mu^{2} \mathrm{t}}\right\}}{1-\left[\Phi\left[\sqrt{\left.\left.\frac{\lambda}{\mathrm{t}}\left(\frac{\mathrm{t}}{\mu}-1\right)\right]+\mathrm{e}^{2 \lambda / \mu} \Phi\left[-\sqrt{\frac{\lambda}{\mathrm{t}}}\left(1+\frac{\mathrm{t}}{\mu}\right)\right]\right]}\right.\right.}$.

O PCPO (5.2.1) para o modelo de estatísticas de ordem gaussiana inversa é dado por,

$$
\mathrm{p}\left(\mathrm{x}_{\mathrm{i}+1} / \mathrm{x}_{\mathrm{i}}\right)=\iint \frac{\frac{\sqrt{\lambda}}{\sqrt{2 \pi}} \mathrm{x}_{\mathrm{i}+1}-3 / 2 \exp \left\{-\frac{\lambda\left(\mathrm{x}_{\mathrm{i}+1}-\mu\right)^{2}}{2 \mu^{2} \mathrm{x}_{\mathrm{i}+1}}\right\}}{1-\left[\Phi\left[\sqrt{\frac{\lambda}{\mathrm{x}_{\mathrm{i}+1}}}\left(\frac{\mathrm{t}}{\mu}-1\right)\right]+\mathrm{e}^{2 \lambda / \mu} \Phi\left[-\sqrt{\frac{\lambda}{\mathrm{x}_{\mathrm{i}+1}}}\left(1+\frac{\mathrm{x}_{\mathrm{i}+1}}{\mu}\right)\right]\right]} \mathrm{p}\left(\mu, \lambda \backslash \mathrm{D}_{\mathrm{x}_{\mathrm{i}}}\right) \mathrm{d} \lambda \mathrm{d} \mu
$$

Os PCPO dos modelos de estatísticas de ordem log-normal e gaussiana inversa, podem ser aproximados por um estimador Monte Carlo, utilizando-se o amostrador de Gibbs e Metropolis-Hastings.

Para seleção dos modelos, podemos "plotar" $c_{i}$ versus $i$, para $\mathrm{i}=2, . ., \mathrm{n}$, para cada modelo. $\mathrm{O}$ maior dos $\mathrm{c}_{\mathrm{i}}$ em média é, o melhor modelo.

Como comentamos na seção (5.1), o critério de verossimilhança preditiva Bayesiano seleciona o modelo, que maximiza a verossimilhança $\mathrm{c}(\ell)=\prod_{\mathrm{i}=1}^{\mathrm{n}} \mathrm{c}_{\mathrm{i}}(\ell)$, onde $\ell$ indexa o $\ell$-ésimo modelo de confiabilidade. Neste caso, $(\ell=1, \ldots 6),(\ell=1$ modelo de estatísticas de ordem gama generalizada, $\ell$ $=2$ Weibul, $\ell=3$ gama, $\ell=4$ exponencial, $\ell=5 \log$-normal, $\ell=6$ gaussiana inversa). 
Neste trabalho, selecionamos também o modelo que minimiza a soma dos erros relativos, denotada neste capítulo por RE. A soma dos erros relativos para os modelos que utilizam processos de Poisson nãohomogêneo é dada pela expressão,

$$
\mathrm{RE}=\frac{\sum_{\mathrm{i}=1}^{\mathrm{n}}\left(\mathrm{n}_{\mathrm{i}}-\hat{\mathrm{m}}\left(\mathrm{x}_{\mathrm{i}}\right)\right)^{2}}{\hat{\mathrm{m}}\left(\mathrm{x}_{\mathrm{i}}\right)},
$$

onde $\mathrm{n}_{\mathrm{i}}$ é o número de erros no software observados no i-ésimo intervalo de tempo e $\hat{\mathrm{m}}\left(\mathrm{x}_{\mathrm{i}}\right)$ é um estimador Bayesiano da função de valor médio.

Por exemplo, considere-se o modelo de estatísticas de ordem exponencial, com função de valor médio dada por,

$$
m(t)=\theta\left(1-e^{-\beta t}\right) .
$$

Considerando as amostras geradas pelo amostrador de Gibbs e Metropolis-Hastings, aproximamos a função de valor médio (5.2.5) por um estimador Monte Carlo dado por,

$$
\hat{\mathrm{m}}\left(\mathrm{x}_{\mathrm{i}}\right)=\frac{2}{\mathrm{RS}} \sum_{\mathrm{r}=1}^{\mathrm{R}} \sum_{\mathrm{s}=\frac{\mathrm{S}}{2}+1}^{\mathrm{S}} \theta^{(\mathrm{r}, \mathrm{s})}\left(1-\mathrm{e}^{-\beta^{(\mathrm{r}, \mathrm{s})} \mathrm{x}_{\mathrm{i}}}\right) .
$$

Para encontrar a soma dos erros relativos do modelo de estatísticas de ordem exponencial, considera-se (5.2.6) em (5.2.4).

Para calcular a soma dos erros relativos RE (5.2.4) para os modelos de estatísticas de ordem Weibul, gama, gama-generalizada, log-normal e gaussiana inversa, aproximamos as funções de valor médio (4.1.7), (4.1.8), (4.1.10), (4.1.12) e (4.1.14), respectivamente, por um estimador Monte Carlo e, substituímos esses estimadores, em (5.2.4) para cada modelo. 


\section{Capítulo 6}

\section{EXEMPLOS DE APLICAÇÕES}

\subsection{Exemplo 1}

Considere o modelo de Moranda (1975), com função intensidade dada por:

$$
\lambda_{\mathrm{i}}=\lambda \mathrm{k}_{1}^{\mathrm{i}-1} \mathrm{i}=1,2, \ldots
$$

onde $\lambda>0$ e $0<\mathrm{k}_{1}<1$ e $\lambda_{\mathrm{i}}$ é a taxa de falha do modelo de Moranda para o i-ésimo intervalo de tempo.

Uma aplicação do modelo de processo de Poisson homogêneo com função intensidade (6.1.1) é dada no estudo do número de falhas de um software observadas em um intervalo de tempo $(0, \mathrm{t}]$. Neste caso, desejamos estimar os parâmetros $\lambda$ (valor médio de falhas do software) e $k_{1}$ (probabilidade de um software falhar no i-ésimo intervalo de tempo). 
Na tabela (1), temos os dados introduzidos por Goel (1985), que se referem ao número de falhas por hora de um software desenvolvido pelo Laboratório Bell, testado por um período de 25 horas.

Tabela 1. Números de falhas por hora de um software testado por 25 horas.

\begin{tabular}{|c|c|c|c|}
\hline $\begin{array}{l}\text { Tempo de teste } \\
\text { do software }\end{array}$ & $\begin{array}{l}\text { Número de falhas } \\
\text { do software por hora }\end{array}$ & $\begin{array}{l}\text { Tempo de teste } \\
\text { do software }\end{array}$ & $\begin{array}{l}\text { Número de falhas do } \\
\text { software por hora }\end{array}$ \\
\hline 1 & 27 & 14 & 5 \\
2 & 16 & 15 & 5 \\
3 & 11 & 16 & 6 \\
4 & 10 & 17 & 0 \\
5 & 11 & 18 & 5 \\
6 & 7 & 19 & 1 \\
7 & 2 & 20 & 1 \\
8 & 5 & 21 & 2 \\
9 & 3 & 22 & 1 \\
10 & 1 & 23 & 2 \\
11 & 4 & 24 & 1 \\
12 & 7 & 25 & \\
13 & 2 & & \\
\hline
\end{tabular}

\section{Análise Clássica}

Nesta seção, aplicamos aos dados da tabela (1), a metodologia de análise clássica baseada na aproximação normal assintótica dos estimadores de máxima verossimilhança, proposta no capítulo 2 .

Considerando-se os dados da tabela (1) e as expressões descritas em (2.1.4), os estimadores de máxima verossimilhança para $\lambda$ e $k_{1}$ (obtido pelo método iterativo de Newton-Rapshon) são dados por: $\hat{\lambda}=16.67257$ e $\hat{\mathrm{k}}_{1}=0.88285$.

A matriz de variância e covariância assintótica de $\hat{\lambda}$ e $\hat{\mathrm{k}}_{1}$, ou seja, a inversa da matriz de informação observada (2.1.7), é dada por, 


$$
I^{-1}\left(\lambda, \mathrm{k}_{1}\right)=\left(\begin{array}{cc}
4.490164 & -0.02032 \\
-0.02032 & 0.000168
\end{array}\right)
$$

Intervalos de confiança $95 \%$ aproximados para $\lambda$ e $\mathrm{k}_{1}$, considerando a aproximação normal assintótica (2.1.6) dos estimadores de máxima verossimilhança $\hat{\lambda}$ e $\hat{\mathrm{k}}_{1}$, são dados por $(0.85739 ; 0.908313)$ e $(12.5191$; 20.82553), respectivamente.

\section{Análise Bayesiana}

Como visto no capítulo 3, em inferência Bayesiana, as inferências sobre os parâmetros de interesse são baseadas nas suas densidades a posteriori marginais.

Para inferir dos parâmetros $\lambda$ e $\mathrm{k}_{1}$, considerando-se o conjunto de dados da tabela (1) e a densidade a priori informativa conjunta (3.4.2), aproximamos as densidades a posteriori marginais de $\lambda$ e $\mathrm{k}_{1}$, utilizando os algoritmos Metropolis-Hastings e amostrador de Gibbs.

Geração de Amostras Aleatórias das Densidades Condicionais de $\lambda$ e $\mathrm{k}_{1}$ pelos Algoritmos Amostrador de Gibbs e Metropolis-Hastings

Através de uma análise preliminar dos dados da tabela (1) obtemos os valores das constantes $b_{1}, b_{2}, c_{1}$ e $c_{2}$ das densidades condicionais de $\lambda$ e $\mathrm{k}_{1}$ para o algoritmo amostrador de Gibbs e Metropolis-Hastings dadas em (3.4.4) e (3.4.6) respectivamente. Os valores encontrados são

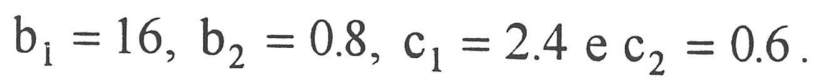

Considerando as densidades condicionais de $\lambda(3.4 .4)$ e de $k_{1}$ (3.4.6) com $b_{1}=16, b_{2}=0.8, c_{1}=2.4 e^{2} c_{2}=0.6$, geramos, pelos 
amostradores de Gibbs e de Metropolis-Hastings, 10 seqüências de 1700 pontos amostrais para cada variável com 10 pares de valores iniciais arbitrários de $\lambda$ e $\mathrm{k}_{1}$ dados na tabelas 2 .

Tabela 2. Valores iniciais de $\lambda$ e $\mathrm{k}_{1}$ para as seqüências do algoritmo amostrador de Gibbs e Metropolis-Hastings.

\begin{tabular}{|c|c|c|}
\hline Sequências & $\lambda$ inicial & $\mathrm{k}_{1}$ inicial \\
\hline 1 & 15.0 & 0.45 \\
\hline 2 & 16.0 & 0.50 \\
\hline 3 & 17.0 & 0.60 \\
\hline 4 & 18.5 & 0.70 \\
\hline 5 & 19.0 & 0.80 \\
\hline 6 & 20.2 & 0.92 \\
\hline 7 & 16.0 & 0.95 \\
\hline 8 & 20.1 & 0.98 \\
\hline 9 & 15.3 & 0.99 \\
\hline 10 & 19.5 & 1.00 \\
\hline
\end{tabular}

Para a seleção da amostra, consideramos em cada seqüência os 100 pontos amostrais finais para $\lambda$ e $\mathrm{k}_{1}$ resultando em uma amostra de 1000 pontos.

Para verificar a independência dos dados construímos o gráfico da correlação dos 1000 pontos amostrais de $\lambda$ e $k_{1}$, ver figuras (1a) e (lb), respectivamente. 


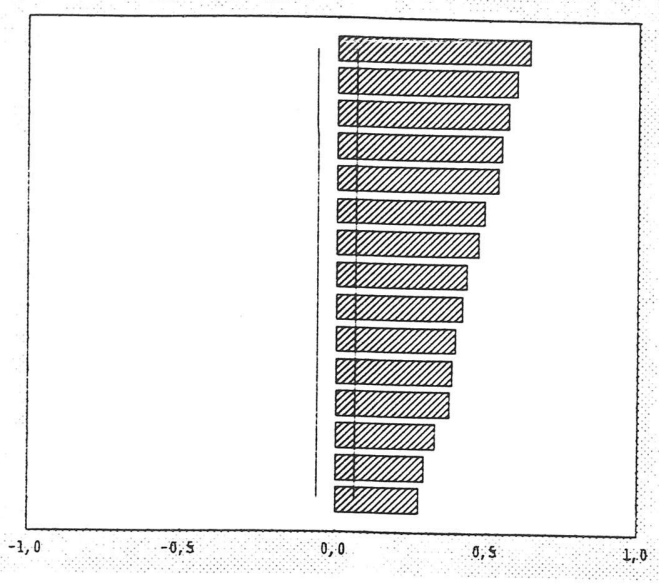

(1a)

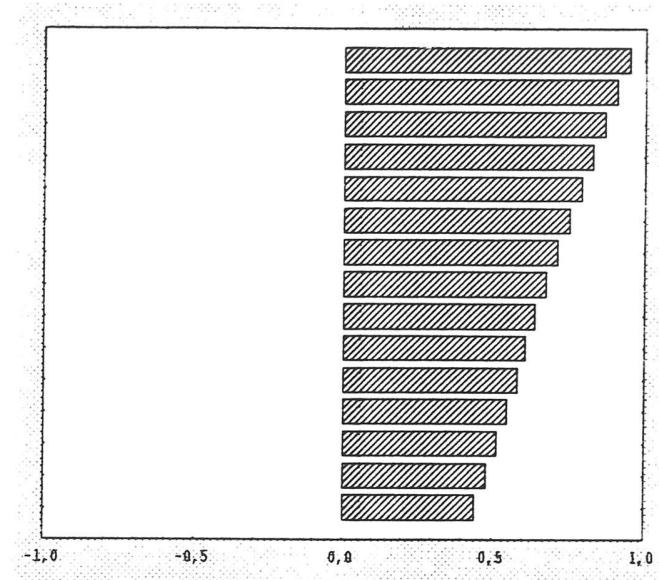

(1b)

Figura 1. Correlação da amostra gerada pelo amostrador de Gibbs com Metropolis de $\lambda$ e $\mathrm{k}_{1}$, selecionada considerando-se os 100 pontos finais de cada sequência.

Observamos através da figura (1) uma alta correlação entre os dados da amostra selecionada utilizando-se os 100 pontos finais de cada seqüência. Portanto devemos considerar outro método de seleção.

Assim, em cada seqüência, desprezamos os primeiros 200 valores e dos 1500 pontos restantes selecionamos 100 pontos, tomados de 15 em 15 , resultando em uma amostra final de 1000 pontos amostrais. Desta forma, obtemos independência dos dados da amostra, ver figura (2).

Na figura (2), temos os gráficos da correlação da amostra de $\lambda \mathrm{e} \mathrm{k}_{1}$, selecionada desprezando-se os 200 valores iniciais e escolhendo de $15 \mathrm{em}$ 15 pontos dos 1500 pontos restantes. Ver figuras (2a) e (2b), respectivamente. 


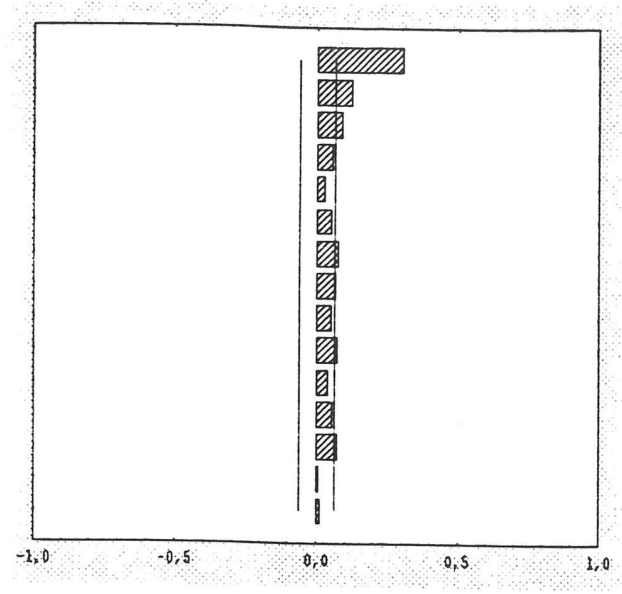

(2a)

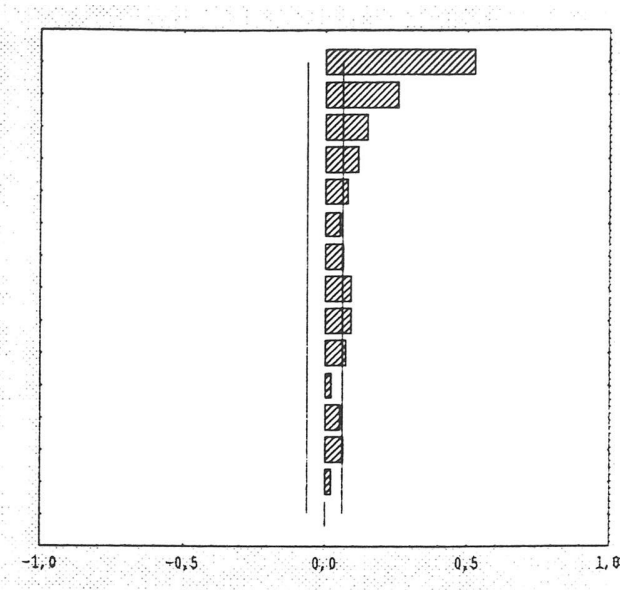

(2b)

Figura 2. Correlação da amostra de $\lambda$ e $k_{1}$, selecionada desprezando os 200 valores iniciais de cada sequêencia e selecionando-se os 1500 pontos restantes de 15 em 15 .

Utilizando as amostras geradas das densidades condicionais de $\lambda$ (3.4.4) e $k_{1}$ (3.4.6), pelos algoritmos amostrador de Gibbs e MetropolisHastings, contruímos os gráficos das densidades a posteriori marginais para $\lambda$ e $k_{1}$, ver figuras (3a) e (3b), respectivamente.

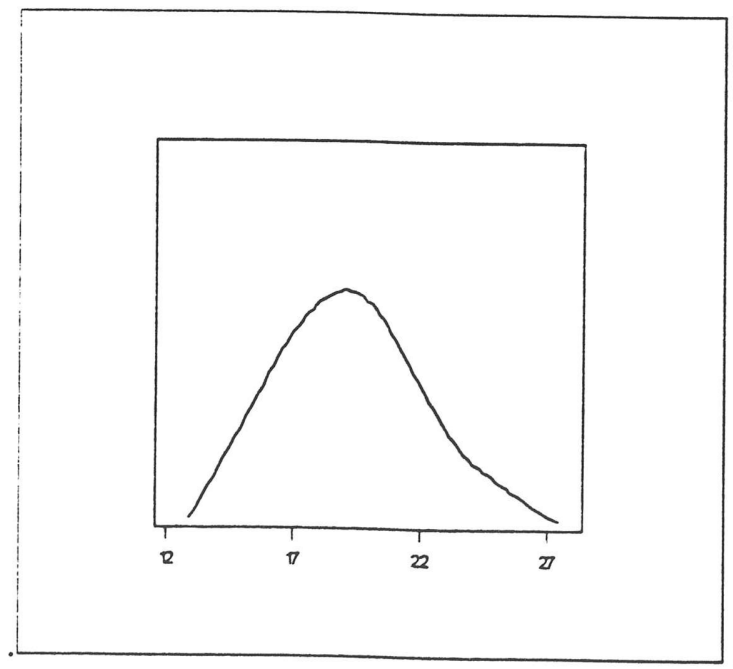

(a)

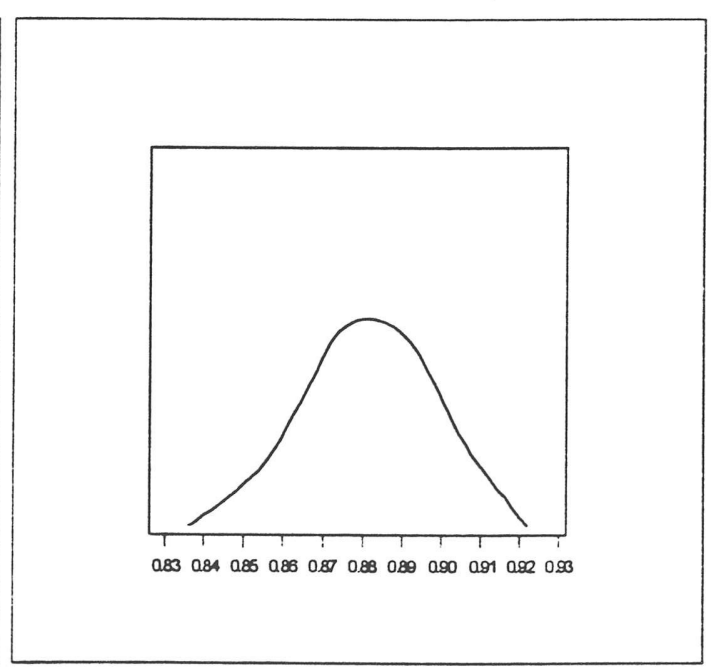

(b)

Figura 3. Densidades a Posteriori Marginais de $\lambda$ e $\mathrm{k}_{1}$, respectivamente, obtidas pelo algoritmo amostrador de Gibbs e Metropolis-Hastings. 
As modas dessas densidades a posteriori marginais são dadas por $\tilde{\lambda}=18.10$ e $\tilde{\mathrm{k}}_{1}=0.887$.

Considerando as amostras geradas das densidades condicionais de $\lambda$ (3.4.4) e de $k_{1}$ (3.4.6) pelo algoritmo de Gibbs com Metropolis, calculamos, os estimadores Monte Carlo para os parâmetros de interesse $\lambda$ e $\mathrm{k}_{1}, \mathrm{E}(\hat{\lambda} /$ dados $)=18.1533$ e $\mathrm{E}\left(\hat{\mathrm{k}}_{1} /\right.$ dados $)=0.8821$, respectivamente.

Intervalos de credibilidade $95 \%$ Bayesianos para $\lambda$ e $\mathrm{k}_{1}$ são dados por: $[14.784 ; 23.8602]$ e $[0.8569 ; 0.9069]$ respectivamente.

A convergência (em distribuição) do algoritmo de Gibbs com Metropolis é verificada pelo critério de Gelman e Rubin (1992).

Os fatores de Redução de Escala para $\lambda$ e $k_{1}$ são dados por: $\mathrm{R}(\lambda)=1.00131$ e $\mathrm{R}\left(\mathrm{k}_{1}\right)=1.0099$. A taxa de aceitação do algoritmo de Metropolis-Hastings é 49.99\%. Observe-se que $\mathrm{R}$ (fator de redução de escala), aproxima-se de 1 com significância de duas casas decimais. Portanto é razoável considerarmos 17000 iterações para obter a convergência do algoritmo de Gibbs com Metropolis para esse modelo.

\section{Comparação entre as Estimativas Pontuais}

Na tabela (3), temos os estimadores de máxima verossimilhança e os estimadores de Monte Carlo de $\lambda$ e $k_{1}$, utilizando as amostras geradas pelo algoritmo de Gibbs com Metropolis.

Tabela 3. Estimadores de máxima verossimilhança e estimadores de Monte Carlo utilizando as amostras geradas pelos algoritmos de Gibbs com Metropolis para os parâmetros $\lambda$ e $\mathrm{k}_{1}$.

\begin{tabular}{|c|c|c|}
\hline Parâmetro & E.M.V. & $\begin{array}{l}\text { Estimador Monte Carlo } \\
\text { (amostrador de Gibbs) }\end{array}$ \\
\hline$\lambda$ & 16.67257 & 18.1533 \\
$\mathrm{k}_{1}$ & 0.88258 & 0.8821 \\
\hline
\end{tabular}


Observamos que as estimativas pontuais para 0 parâmetro $\lambda$ são razoavelmente próximas e para o parâmetro $\mathrm{k}_{1}$ são próximas, considerando o método clássico e Bayesiano com densidades a priori informativas.

\section{Comparação entre as Estimativas por Intervalos}

Para facilitar a comparação, construímos a tabela (4), onde temos inferências por intervalos para os parâmetros $\lambda$ e $\mathrm{k}_{1}$, obtidas através das análises clássica e Bayesiana considerando densidade a priori informativas.

Tabela 4. Intervalos de Confiança 95\% e Intervalos de Credibilidade Bayesianos $95 \%$ para $\lambda$ e $\mathrm{k}_{1}$.

\begin{tabular}{|c|c|c|}
\hline Parâmetros & $\begin{array}{c}\text { Normalidade Assintótica } \\
\text { de } \lambda \mathrm{e}_{1}\end{array}$ & $\begin{array}{c}\text { Intervalo de Credibilidade } \\
\text { Bayesiano } \\
\text { (amostrador de Gibbs) }\end{array}$ \\
\hline$\lambda$ & $(12.5191 ; 20.82553)$ & $(14.784 ; 23.458)$ \\
$\mathrm{k}_{1}$ & $(0.85739 ; 0.908313)$ & $(0.8591 ; 0.9067)$ \\
\hline
\end{tabular}

Os intervalos para $\mathrm{k}_{1}$ são próximos e para $\lambda$ são razoavelmente próximos para o método clássico e Bayesiano, observamos uma amplitude menor dos intervalos para o caso Bayesiano, indicando uma precisão melhor, considerando densidades a priori informativas.

\section{Forma Generalizada do Modelo de Moranda (Achcar, 1995)}

Como comentamos no Capítulo 2, a forma generalizada do modelo de Moranda pode ser feita inserindo um parâmetro $\mathrm{k}_{2}$ e uma reparametrização $\lambda_{\mathrm{a}}=\frac{\lambda}{\mathrm{k}_{1}}$ na função intensidade (6.1.1). Assim temos, 
Uma aplicação do modelo de processo de Poisson homogêneo com função intensidade (6.1.2) é utilizada no estudo do número de falhas de um software observadas durante um intervalo de tempo $(0, t]$. Nesse caso, desejamos estimar os parâmetros $\lambda_{\mathrm{a}}, \mathrm{k}_{1}$ e $\mathrm{k}_{2}$.

Devemos ressaltar que a reparametrização $\lambda_{\mathrm{a}}=\frac{\lambda}{\mathrm{k}_{1}}$ é feita somente para facilitar os cálculos computacionais

\section{Análise Clássica}

Tomando-se os dados da tabela (1) e a expressão dada em (2.2.3), obtemos os estimadores de máxima verossimilhança para $\lambda_{a}, k_{1}$ e $k_{2}$ (obtidos pelo método de Newton), dados por: $\hat{\lambda}_{\mathrm{a}}=252.516, \hat{\mathrm{k}}_{1}=0.10 \mathrm{e}$ $\hat{\mathrm{k}}_{2}=0.248$.

A matriz de informação observada de $\hat{\lambda}_{a}, \hat{\mathrm{k}}_{1}$ e $\hat{\mathrm{k}}_{2}$ (2.2.5) é dada por,

$$
\mathrm{I}\left(\lambda_{\mathrm{a}}, \mathrm{k}_{1}, \mathrm{k}_{2}\right)=\left(\begin{array}{ccc}
46605.377 & -17.5693 & -13.10554 \\
-17.56930 & 0.006797 & 0.005251 \\
-13.10554 & 0.005251 & 0.004356
\end{array}\right)
$$

Intervalos de confiança $95 \%$ aproximados para $\lambda_{\mathrm{a}}, \mathrm{k}_{1}$ e $\mathrm{k}_{2}$, considerando-se a aproximação normal assintótica (2.2.4) dos E.M.V. são dados por $(-170.6142 ; 675.6463),(-0.0616 ; 0.2616)$ e $(0.118635 ; 0.37736)$ respectivamente.

Observemos que os intervalos de confiança para os parâmetros $\lambda_{a} e$ $\mathrm{k}_{1}$ apresentam valores "absurdos"; pois $\lambda_{\mathrm{a}}$ é um número restrito maior que $0\left(\lambda_{\mathrm{a}}>0\right)$ e $\mathrm{k}_{1}$ é a probabilidade de o software falhar no i-ésimo intervalo de tempo, portanto, $0<\mathrm{k}_{1}<1$. 


\section{Análise Bayesiana}

Para inferir dos parâmetros $\lambda_{\mathrm{a}}, \mathrm{k}_{1} \mathrm{e} \mathrm{k}_{2}$, considerando o conjunto de dados da tabela (1) e as densidades a priori informativas (3.6.1), aproximamos as densidades a posteriori marginais de $\lambda_{a}, k_{1}$ e $k_{2}$, utilizando os algoritmos amostrador de Gibbs e Metropolis-Hastings.

\section{Geração de Amostras Aleatórias das Densidades} Condicionais de $\lambda$ e $k_{1}$ pelos Algoritmos Amostrador de Gibbs e Metropolis-Hastings

Fazendo uma análise preliminar dos dados da tabela (1), obtemos os valores das constantes $b_{1}, b_{2}, c_{1}, c_{2}, \mu_{0}$ e $\sigma_{0}^{2}$ descritas em (3.5.1), através da média e variabilidade dos parâmetros $\lambda_{\mathrm{a}}, \mathrm{k}_{1}$ e $\mathrm{k}_{2}$.

Por exemplo, assumindo uma densidade a priori gama, denotada por $\Gamma\left(b_{1}, b_{2}\right)$ para $\lambda_{a}$ e considerando o estimador de máxima verossimilhança $\hat{\lambda}_{\mathrm{a}}=252.516$, temos: $\mathrm{E}\left(\lambda_{\mathrm{a}}\right)=\frac{\mathrm{b}_{1}}{\mathrm{~b}_{2}}=250$ e $\operatorname{Var}\left(\lambda_{\mathrm{a}}\right)=\frac{\mathrm{b}_{1}}{\mathrm{~b}_{2}^{2}}=250$,

Desta forma, encontramos os valores de $b_{1}$ e $b_{2}$, dados por $b_{1}=250$ e $b_{2}=1$. Utilizando o mesmo procedimento para encontrar as constantes $\mathrm{c}_{1}, \mathrm{c}_{2}, \mu_{0}$ e $\sigma_{0}^{2}$ das densidades condicionais dos parâmetros $\mathrm{k}_{1} \mathrm{e}$ $\mathrm{k}_{2}$ temos, $\mathrm{c}_{1}=1.5, \mathrm{c}_{2}=13, \mu_{0}=0.25$ e $\sigma_{0}=0.04$.

Substituindo os valores das constantes nas densidades condicionais de $\lambda_{\mathrm{a}}$ (3.5.3), $\mathrm{k}_{1}$ (3.5.5) e $\mathrm{k}_{2}$ (3.5.7), geramos, pelos amostradores de Gibbs e Metropolis-Hastings, 10 seqüências de 1700 pontos para cada variável com 10 valores iniciais arbitrários de $\lambda_{\mathrm{a}}, \mathrm{k}_{1}$ e $\mathrm{k}_{2}$.

Em cada seqüência, desprezamos os primeiros 200 valores e dos 1500 restantes selecionamos 100 pontos tomados de 15 em 15, resultando em uma amostra final de 1000 pontos. A seleção dessa amostra foi realizada de forma a obter independência e uma baixa correlação entre os dados. 
Utilizando as amostras geradas das densidades condicionais de $\lambda_{\mathrm{a}}$ (3.6.4), $\mathrm{k}_{1}$ (3.6.6) e $\mathrm{k}_{2}$ (3.6.8), pelos algoritmos amostrador de Gibbs e Metropolis-Hastings, construímos os gráficos das densidades a posteriori marginais para $\lambda_{a}, k_{1}$ e $k_{2}$, ver figuras (4a), (4b) e (4c), respectivamente.

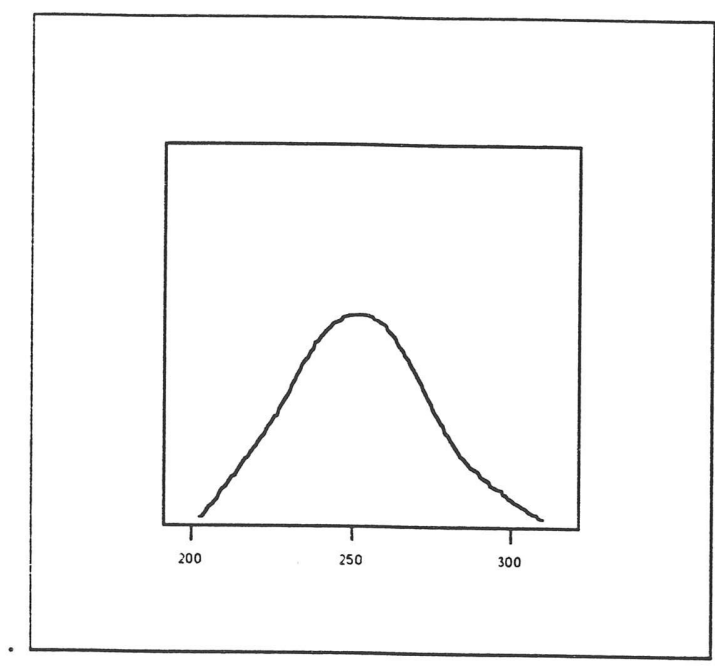

(a)

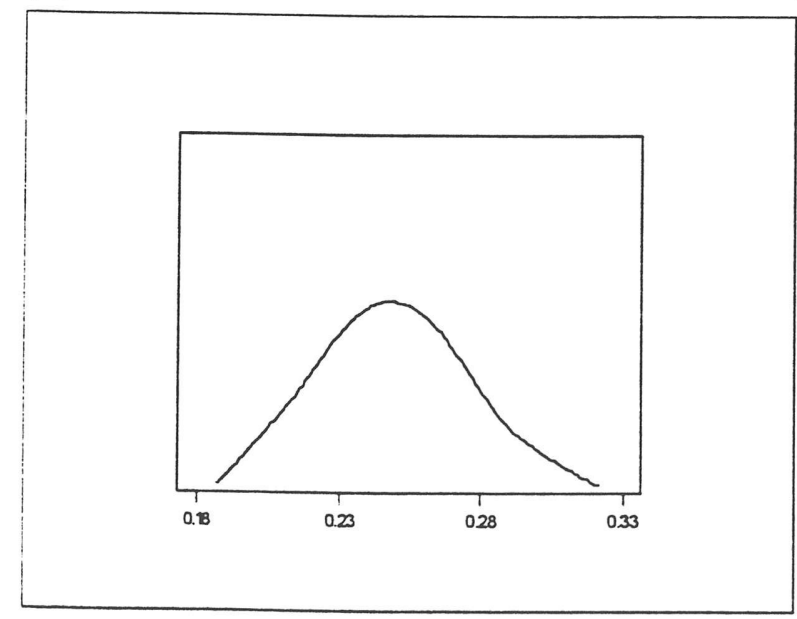

(c)

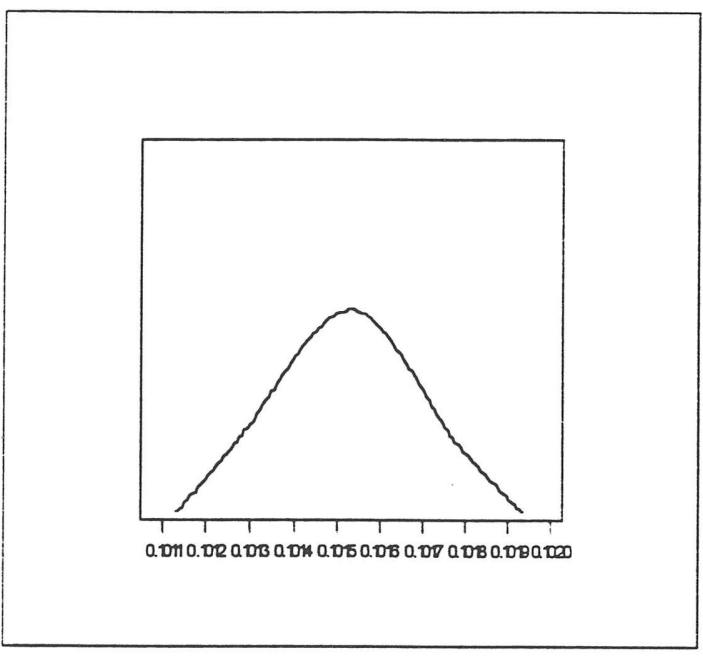

(b)

Figura 4. Densidades a Posteriori Marginais de $\lambda_{\mathrm{a}}, \mathrm{k}_{1}$ e $\mathrm{k}_{2}$ obtidas pelo algoritmo amostrador de Gibbs e Metropolis-Hastings.

Na tabela (5), temos os sumários a posteriori dos parâmetros $\lambda_{\mathrm{a}}, \mathrm{k}_{1}$ e $\mathrm{k}_{2}$, considerando as amostras geradas pelo algoritmo amostrador de Gibbs com Metropolis. 
Tabela 5. Sumários a posteriori de $\lambda_{\mathrm{a}}, \mathrm{k}_{1} \mathrm{e} \mathrm{k}_{2}$ obtidos pelo algoritmo amostrador de Gibbs com Metropolis

\begin{tabular}{|c|cccc|}
\hline Parâmetros & Média & Mediana & Desvio Padrão & $\begin{array}{c}\text { Intervalo de Credibilidade } \\
\text { Bayesiano 95\% }\end{array}$ \\
\hline$\lambda_{\mathrm{a}}$ & 249.91 & 249.70 & 14.58 & $(222.004 ; 280.024)$ \\
$\mathrm{k}_{1}$ & 0.10152 & 0.1013 & 0.01141 & $(0.07854 ; 0.12640)$ \\
$\mathrm{k}_{2}$ & 0.24816 & 0.24764 & 0.02106 & $(0.20988 ; 0.27989)$ \\
\hline
\end{tabular}

A convergência (em distribuição) do algoritmo amostrador de Gibbs com Metropolis é verificada pelo critério de Gelman e Rubin (1992).

Os fatores de Redução de Escala para $\lambda_{a}, k_{1}$ e $k_{2}$ são dados por: $R\left(\lambda_{a}\right)=1.00110$ e $R\left(k_{1}\right)=0.99999 R\left(k_{2}\right)=1.0099$. Observe que $R$ (fator de redução de escala), aproxima-se de 1 com significância de duas casas decimais para $\lambda_{\mathrm{a}}$ e $\mathrm{k}_{2}$. Portanto, é razoável considerarmos 17000 iterações para obter a convergência do algoritmo de Gibbs com Metropolis para esse modelo.

\section{Comparação das Estimativas Pontuais}

Na tabela (6), temos os estimadores de máxima verossimilhança e estimadores Monte Carlo considerando as amostras geradas pelo algoritmo amostrador de Gibbs com Metropolis para $\lambda_{\mathrm{a}}, \mathrm{k}_{1}$ e $\mathrm{k}_{2}$. 
Tabela 6. Estimadores de máxima verossimilhança e estimadores Monte Carlo considerando as amostras geradas pelo algoritmo de Gibbs com Metropolis para $\lambda_{\mathrm{a}}, \mathrm{k}_{1}$ e $\mathrm{k}_{2}$.

\begin{tabular}{|c|c|c|}
\hline Parâmetros & E.M.V. & $\begin{array}{c}\text { Estimadores Monte Carlo } \\
\text { Bayesianos }\end{array}$ \\
\hline$\lambda_{\mathrm{a}}$ & 252.516 & 249.91 \\
$\mathrm{k}_{1}$ & 0.1000 & 0.10152 \\
$\mathrm{k}_{2}$ & 0.2480 & 0.24816 \\
\hline
\end{tabular}

Observamos que as estimativas pontuais de $\mathrm{k}_{1}$ e $\mathrm{k}_{2}$ são próximas e, para $\lambda_{\mathrm{a}}$ são razoavelmente próximas, para o método clássico e Bayesiano, considerando densidades a priori informativas.

\section{Comparação entre as Estimativas por Intervalos}

$\mathrm{Na}$ tabela (7), mostramos as inferências por intervalos para os parâmetros $\lambda_{\mathrm{a}}, \mathrm{k}_{1} \mathrm{e} \mathrm{k}_{2}$, obtidas através das análises clássica e Bayesiana, considerando densidades a priori informativas.

Tabela 7. Intervalos de confiança $95 \%$ e Intervalos de Credibilidade Bayesianos $95 \%$ para $\lambda_{\mathrm{a}}, \mathrm{k}_{1}$ e $\mathrm{k}_{2}$.

\begin{tabular}{|c|c|c|}
\hline Parâmetros & $\begin{array}{c}\text { Normalidade assintótica } \\
\text { de } \hat{\lambda}_{\mathrm{a}}, \hat{\mathrm{k}}_{1} \mathrm{e} \hat{\mathrm{k}}_{2}\end{array}$ & $\begin{array}{c}\text { Intervalo de } \\
\text { Credibilidade Bayesiano } \\
\text { (amostrador de Gibbs) }\end{array}$ \\
\hline$\lambda_{\mathrm{a}}$ & $(-170.614 ; 675.6436)$ & $(222.004 ; 280.024)$ \\
$\mathrm{k}_{1}$ & $(-0.06160 ; 0.26161)$ & $(0.07854 ; 0.12640)$ \\
$\mathrm{k}_{2}$ & $(0.118635 ; 0.37736)$ & $(0.20988 ; 0.27989)$ \\
\hline
\end{tabular}


Através da tabela (7), observamos uma precisão significativamente melhor da inferência por intervalos no caso Bayesiano do que no caso clássico. Os intervalos clássicos para $\lambda_{\mathrm{a}} \mathrm{e} \mathrm{k}_{1}$ apresentam valores absurdos para esses parâmetros, pois $\lambda_{\mathrm{a}}>0$ e $0<\mathrm{k}_{1}<1$, indicando que uma reparametrização para $\lambda_{\mathrm{a}}$ e $\mathrm{k}_{1}$ torna-se necessária para a precisão das estimativas por intervalo.

\section{Discriminação Bayesiana dos Processos de Poisson Homogêneo}

A seguir, conduziremos a discriminação Bayesiana dos modelos de Moranda (1975) e da forma generalizada do Modelo de Moranda (Achcar, 1995), utilizando as estratégias apresentadas no capítulo 5, seção (5.1).

Na tabela (8), apresentamos as estimativas Bayesianas para a função intensidade $\lambda_{i}$, para $(i=1,2, \ldots, 25)$ com respeito a função de perda média quadrática, considerando as amostras geradas pelo amostrador de Gibbs, para os modelos de Moranda e para a forma generalizada desse modelo. Observamos estimativas mais precisas da taxa de falha para a generalização do modelo de Moranda. 
Tabela 8. Estimadores Monte Carlo da taxa de falhas do modelo de Moranda (6.1.1) e da generalização do modelo de Moranda (6.1.2), considerando-se as amostras de Gibbs e Metropolis-Hastings.

\begin{tabular}{|c|c|c|c|}
\hline $\mathrm{i}$ & $\mathrm{mi}$ & $\begin{array}{r}\lambda_{\mathrm{i}} \\
(6.1 .1)\end{array}$ & $\begin{array}{c}\lambda_{i} \\
(6.1 .2)\end{array}$ \\
\hline 1 & 27 & 16.6724 & 25.2912 \\
\hline 2 & 16 & 14.7277 & 16.4452 \\
\hline 3 & 11 & 13.0087 & 12.3314 \\
\hline 4 & 10 & 11.4941 & 9.8455 \\
\hline 5 & 11 & 10.1562 & 8.2028 \\
\hline 6 & 7 & 8.9768 & 7.0035 \\
\hline 7 & 2 & 7.9346 & 6.0934 \\
\hline 8 & 5 & 7.0144 & 5.3421 \\
\hline 9 & 3 & 6.2014 & 4.8100 \\
\hline 10 & 1 & 5.4775 & 4.3251 \\
\hline 11 & 4 & 4.8754 & 3.9257 \\
\hline 12 & 7 & 4.2913 & 3.5877 \\
\hline 13 & 2 & 3.8001 & 3.2956 \\
\hline 14 & 5 & 3.3645 & 3.0444 \\
\hline 15 & 5 & 2.9784 & 2.8207 \\
\hline 16 & 6 & 2.6355 & 2.6311 \\
\hline 17 & 0 & 2.3351 & 2.4522 \\
\hline 18 & 5 & 2.0684 & 2.2966 \\
\hline 19 & 1 & 1.8344 & 2.1548 \\
\hline 20 & 1 & 1.6234 & 2.0278 \\
\hline 21 & 2 & 1.4388 & 1.9167 \\
\hline 22 & 1 & 1.2752 & 1.8143 \\
\hline 23 & 2 & 1.1305 & 1.7044 \\
\hline 24 & 1 & 1.0042 & 1.6245 \\
\hline 25 & 1 & 0.8874 & 1.5399 \\
\hline
\end{tabular}

Considerando-se novamente os dados da tabela (1), são apresentados os resultados obtidos do valor de predição médio e a soma dos erros de predição para o modelo de Moranda (5.1.8) e para a forma generalizada do modelo de Moranda (5.1.11). 
Na tabela (9), apresentamos as estimativas Bayesianas dos valores de predição e soma dos erros predição para os modelos de Moranda e para a generalização do modelo de Moranda. Comparando os valores encontrados, podemos verificar que a generalização do modelo de Moranda proposta por Achcar (1995), apresenta menor soma dos erros de predição, ou seja proporciona um melhor ajuste aos dados da tabela (1).

Tabela 9. Valor de predição e soma dos erros de predição do modelo Moranda e da generalização do modelo de Moranda.

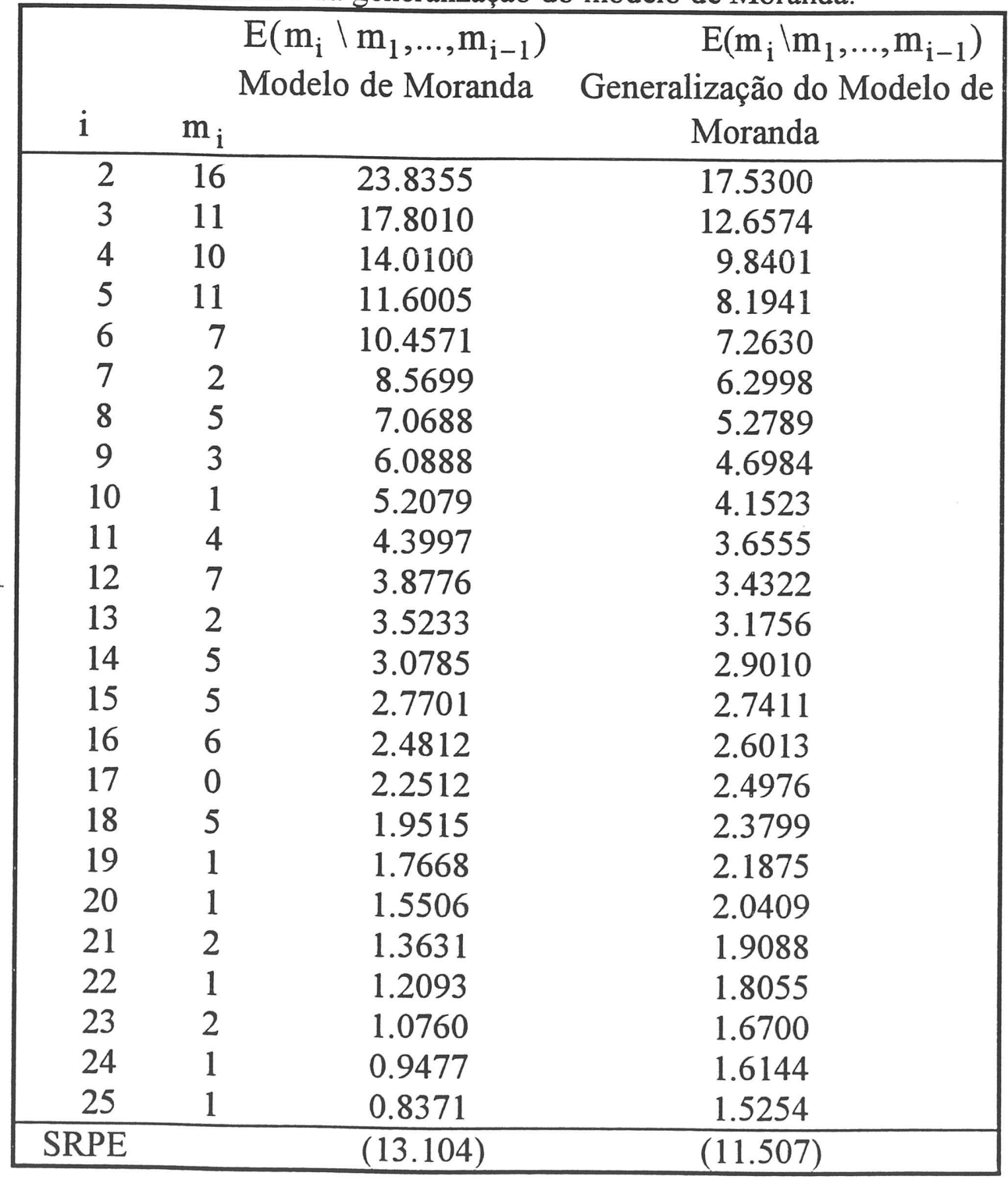


A seguir são apresentadas as densidades preditivas para o cálculo da estratégia de discriminação PCPO dos modelos de Moranda e da generalização do modelo de Moranda.

Utilizando-se os dados da tabela (1) e as amostras geradas pelo amostrador de Gibbs com Metropolis, calculamos os estimadores Monte Carlo para as densidades preditivas do modelo de Moranda (3.4.8) e para a forma generalizada do modelo de Moranda (3.5.9).

A tabela (10) apresenta os valores de $c_{i}=f\left(m_{i} \backslash m_{1}, \ldots, m_{i-1}\right)$ para o modelo de Moranda e para forma generalizada do modelo de Moranda.

Tabela 10. Valores de $c_{i}=f\left(m_{i} \backslash m_{1}, \ldots, m_{i-1}\right)$

$(\mathrm{i}=1, \ldots, 25)$, para o modelo de Moranda e para a generalização do modelo de Moranda.

\begin{tabular}{|l|lll|}
\hline $\mathrm{m}_{\mathrm{i}}$ & $\mathrm{i}$ & $\begin{array}{c}\mathrm{c}_{\mathrm{i}} \\
\text { (modelo 6.1.1) }\end{array}$ & $\begin{array}{c}\mathrm{c}_{\mathrm{i}} \\
\text { (modelo 6.2) }\end{array}$ \\
\hline 27 & 1 & 0.007580 & 0.063681 \\
16 & 2 & 0.087906 & 0.091125 \\
11 & 3 & 0.08512 & 0.106844 \\
10 & 4 & 0.109904 & 0.120416 \\
11 & 5 & 0.111512 & 0.077458 \\
7 & 6 & 0.116557 & 0.145121 \\
2 & 7 & 0.012489 & 0.050014 \\
5 & 8 & 0.126681 & 0.169788 \\
3 & 9 & 0.090144 & 0.152484 \\
1 & 10 & 0.020899 & 0.060144 \\
4 & 11 & 0.177855 & 0.189999 \\
7 & 12 & 0.074512 & 0.043152 \\
2 & 13 & 0.163214 & 0.200741 \\
5 & 14 & 0.121922 & 0.103554 \\
5 & 15 & 0.099945 & 0.090111 \\
6 & 16 & 0.035781 & 0.034345 \\
0 & 17 & 0.103755 & 0.090777 \\
5 & 18 & 0.042155 & 0.057746 \\
1 & 19 & 0.291345 & 0.250458 \\
1 & 20 & 0.321231 & 0.266754 \\
2 & 21 & 0.241091 & 0.264231 \\
1 & 22 & 0.348122 & 0.295263 \\
2 & 23 & 0.351221 & 0.299987 \\
1 & 24 & 0.365844 & 0.298687 \\
1 & 25 & 0.369881 & 0.334125 \\
\hline & $\mathrm{c}(\ell)$ & $4.799 \times 10^{-26}$ & $2.8196 \times 10^{-23}$ \\
\hline & & & \\
\hline
\end{tabular}


A figura (5), mostra os valores de $\mathrm{c}_{\mathrm{i}}$ versus $\mathrm{i}=1, \ldots, 25$ (números de intervalos) para os modelos de Moranda e para a generalização do modelo de Moranda.

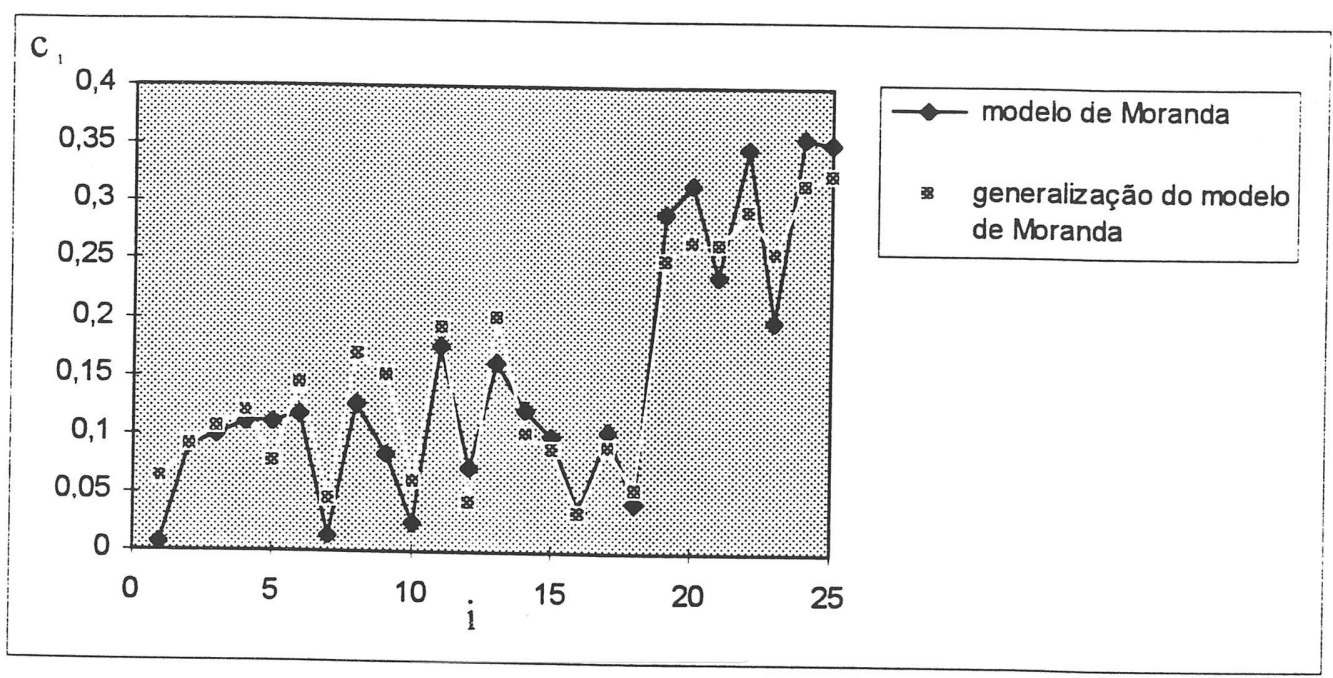

Figura 5. Valores de $c_{i}$ versus $i$ para os modelos de Moranda e para a generalização do modelo de Moranda.

Observando a tabela (10) e a figura (5), notamos que a generalização do modelo de Moranda apresenta em média maiores valores de $c_{i}$ e também maximiza $\quad \mathrm{c}(\ell)=\prod_{\mathrm{i}=1}^{25} \mathrm{f}\left(\mathrm{m}_{\mathrm{i}} \backslash \mathrm{m}_{1}, \ldots, \mathrm{m}_{\mathrm{i}-1}\right)$, indicando que a generalização do modelo de moranda apresenta um melhor ajuste aos dados da tabela (1) do que ao modelo de Moranda.

\section{Algumas Conclusões}

Para o modelo de Moranda, verificamos um concordância razoável entre as inferências obtidas por intervalos e as inferências pontuais, utilizando a metodologia clássica e Bayesiana, assumindo densidades $a$ priori informativas. 
Para a generalização do modelo de Moranda, observa-se uma concordância das estimativas pontuais, considerando a metodologia clássica e Bayesiana.

As estimativas por intervalo apresentam valores absurdos para os parâmetros do modelo (6.1.2), utilizando-se o método de máxima verossimilhança. Assim, para utilizarmos os resultados assintóticos da teoria clássica usual para o modelo (6.1.2) e para o conjunto de dados da tabela (1), reparametrizações tornam-se necessárias para precisão das estimativas.

Quando não podemos utilizar os resultados assintóticos da teoria clássica devido à complexidade do modelo em estudo, e ao conjunto de dados, o uso de métodos Bayesianos aproximados é uma alternativa apropriada.

A título de decidir qual dos dois modelos analisados melhor se ajusta aos dados em estudo, podemos concluir através das estratégias de discriminação utilizadas que a generalização do modelo de Moranda mostrou-se superior ao modelo de Moranda.

Fazendo comparação entre as duas estratégias utilizadas para a seleção de modelo, soma dos erros de predição e PCPO, podemos contatar que há uma concordância entre as mesmas e as duas técnicas mostraram-se eficientes.

A inferência Bayesiana, utilizando o amostrador de Gibbs com Metropolis torna-se muito simples e muito fácil de ser aplicada.

No exemplo seguinte, vamos aplicar a análise Bayesiana para os modelos que utilizam processos de Poisson não homogêneo para modelagem dos dados de falhas de um software. 


\subsection{Exemplo 2}

Neste exemplo, vamos considerar o conjunto de dados da tabela (11), que foram introduzidos por Jelinski e Moranda (1972), consistindo no número de dias entre as 26 falhas que ocorreram durante a fase de produção do software NTDS (em inglês Naval Tactical Data System).

Neste trabalho, processos de Poisson não-homogêneo são considerados para modelar os dados da tabela (11). Os modelos explorados são os de estatísticas de ordem exponencial, gama, Weibul, gama generalizada, log-normal e gaussiana inversa.

Tabela 11. Número de dias entre as 26 falhas que ocorreram na fase de teste do software NTDS $\left(t_{i}=x_{i}-x_{i-1}\right)$ refere-se ao tempo entre falha.

\begin{tabular}{|r|r|r|c|c|c|c|c|c|}
\hline $\mathrm{i}$ & $\mathrm{t}_{\mathrm{i}}$ & $\mathrm{x}_{\mathrm{i}}$ & $\mathrm{i}$ & $\mathrm{t}_{\mathrm{i}}$ & $\mathrm{x}_{\mathrm{i}}$ & $\mathrm{i}$ & $\mathrm{t}_{\mathrm{i}}$ & $\mathrm{x}_{\mathrm{i}}$ \\
\hline 1 & 9 & 9 & 11 & 1 & 71 & 21 & 11 & 116 \\
2 & 12 & 21 & 12 & 6 & 77 & 22 & 33 & 149 \\
3 & 11 & 32 & 13 & 1 & 78 & 23 & 7 & 156 \\
4 & 4 & 36 & 14 & 9 & 87 & 24 & 91 & 247 \\
5 & 7 & 43 & 15 & 4 & 91 & 25 & 2 & 249 \\
6 & 2 & 45 & 16 & 1 & 92 & 26 & 1 & 250 \\
7 & 5 & 50 & 17 & 3 & 95 & & & \\
8 & 8 & 58 & 18 & 3 & 98 & & & \\
9 & 5 & 63 & 19 & 6 & 104 & & & \\
10 & 7 & 70 & 20 & 1 & 105 & & & \\
\hline
\end{tabular}

Assumindo o modelo de estatísticas de ordem gama generalizada na modelagem dos dados da tabela (11), com função de valor médio dada por,

$$
\mathrm{m}(\mathrm{t})=\theta \mathrm{I}_{k}\left(\beta \mathrm{t}^{\alpha}\right)
$$

onde $\mathrm{I}_{k}$ é uma integral gama incompleta dada por, $\mathrm{I}_{k}(\mathrm{~s})=\frac{1}{\Gamma(k)} \int_{0}^{\mathrm{s}} \mathrm{x}^{k-1} \mathrm{e}^{-\mathrm{x}} \mathrm{dx}, \mathrm{e} \alpha>0, \beta>0, \theta>0$ e $k>0$. 
$\mathrm{Na}$ prática, temos interesse em fazer inferências dos parâmetros $\theta, \alpha, \beta$ e $k$ ou funções dos mesmos (por exemplo, taxa de falha, confiabilidade, etc).

A metodologia considerada para fazer inferências dos parâmetros $\theta, \alpha, \beta$ e $k$ são os métodos Bayesianos apresentados no Capítulo 4.

\section{Análise Bayesiana}

Como vimos anteriormente, na metodologia Bayesiana, as inferências sobre os parâmetros de interesse são baseadas nas suas densidades a posteriori marginais, resultando em um procedimento que pode simplificar as interpretações para vários problemas práticos.

Para inferir dos parâmetros $\theta, \alpha, \beta$ e $k$, considerando o conjunto de dados da tabela (11) e as densidade a priori informativas (4.2.2), aproximamos as densidades a posteriori marginais de $\theta, \alpha, \beta$ e $k$, utilizando os algoritmos Metropolis-Hastings e amostrador de Gibbs.

\section{Geração de Amostras Aleatórias das Densidades Condicionais de $\theta, \alpha, \beta$ e $k$ pelos Algoritmos Amostrador de Gibbs e Metrópolis-Hastings}

Considerando os dados da tabela (11), obtemos os estimadores de máxima verossimilhança de $\beta, \theta, k$ e $\alpha$, utilizando o pacote estatístico SAS. Os valores encontrados são $\hat{\beta}=5.1279, \hat{\alpha}=0.2748, \hat{k}=18.201 \quad$ e e $\hat{\theta}=29.4301$. Considerando os estimadores de máxima verossimilhança, obtemos os valores das constantes a,b,c,d,e,f,g e $\mathrm{h}$ das densidades condicionais de $\theta, \alpha, \beta$ e $k$ para o algoritmo amostrador de Gibbs e Metropolis-Hastings. Os valores encontrados são $a=60, b=2, c=10, d=2$, $\mathrm{e}=12, \mathrm{f}=28, \mathrm{~g}=20 \mathrm{e} \mathrm{h}=1$. 
Considerando as densidades condicionais de $\theta, \beta, \alpha$ e $k$, descritas em (4.2.4), (4.2.5), (4.2.6) e (4.2.7) respectivamente, com a=60, b=2, $\mathrm{c}=10$, $\mathrm{d}=2, \mathrm{e}=12, \mathrm{f}=28, \mathrm{~g}=20 \mathrm{e} \mathrm{h}=1$, geramos, pelos amostradores de Gibbs e Metropolis-Hastings, 10 seqüências de 2000 pontos amostrais para cada variável, com 10 valores iniciais arbitrários para $\theta, \alpha, \beta$ e $k$.

Em cada seqüência, desprezamos os primeiros 500 valores e dos 1500 pontos restantes selecionamos 100 pontos, tomados de $15 \mathrm{em} \mathrm{15,} \mathrm{com} \mathrm{o}$ objetivo de obter independência entre os elementos da amostra. Assim, obtém-se uma amostra final de 1000 pontos amostrais.

A convergência (em distribuição) do algoritmo de Gibbs com Metropolis é verificada pelo critério de Gelman e Rubin (1992).

Os fatores de Redução de Escala para $\theta, \alpha, \beta$ e $k$ são dados por: $\mathrm{R}(\theta)=0.9999, \mathrm{R}(\alpha)=1.0099, \mathrm{R}(\beta)=1.0178, \mathrm{R}(k)=1.0048$. Observe-se que $\mathrm{R}$ (fator de redução de escala) aproxima-se de 1 . Portanto é razoável considerarmos 20000 iterações para obter a convergência do algoritmo de Gibbs e Metropolis-Hastings para esse modelo.

A taxa de aceitação do algoritmo de Metropolis-Hastings é 37,71\%, indicando uma aceitação razoável.

Utilizando o amostrador de Gibbs com Metropolis, construímos os gráficos das densidades a posteriori marginais para $\beta, \theta, k \mathrm{e} \alpha$, Ver figuras (6a), (6b), (6c) e (6d), respectivamente. 


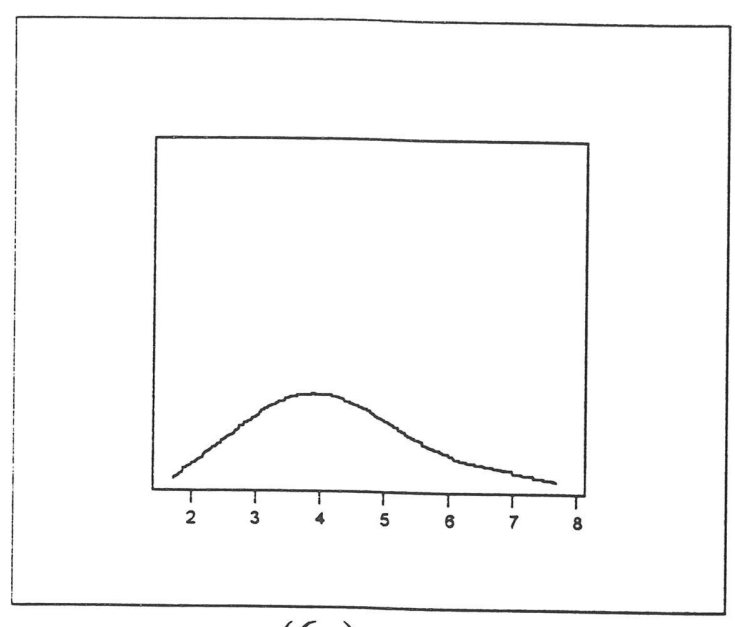

(6a)

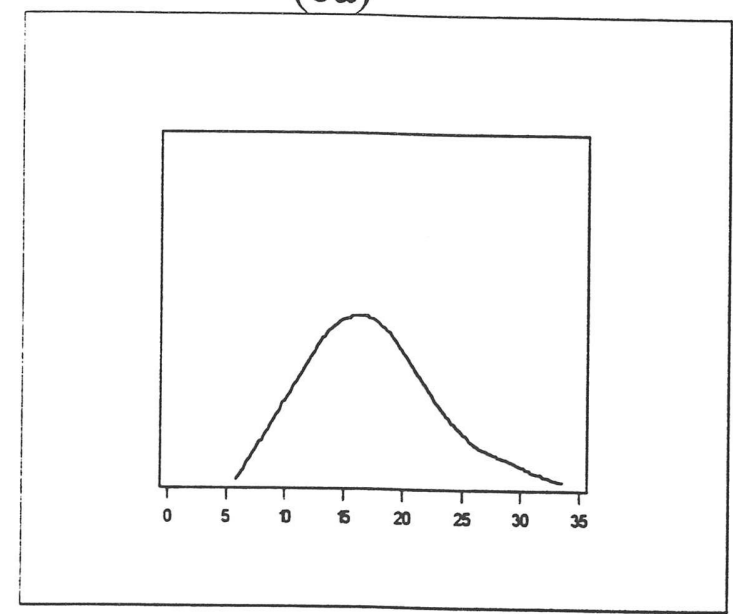

(6c)

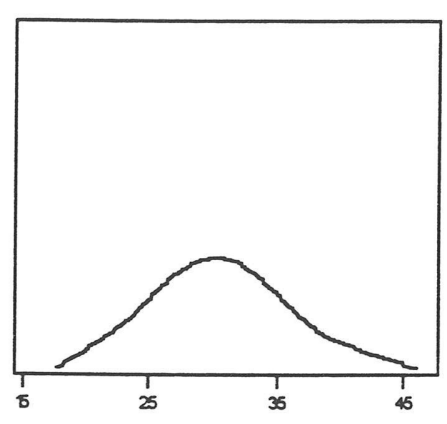

(6b)

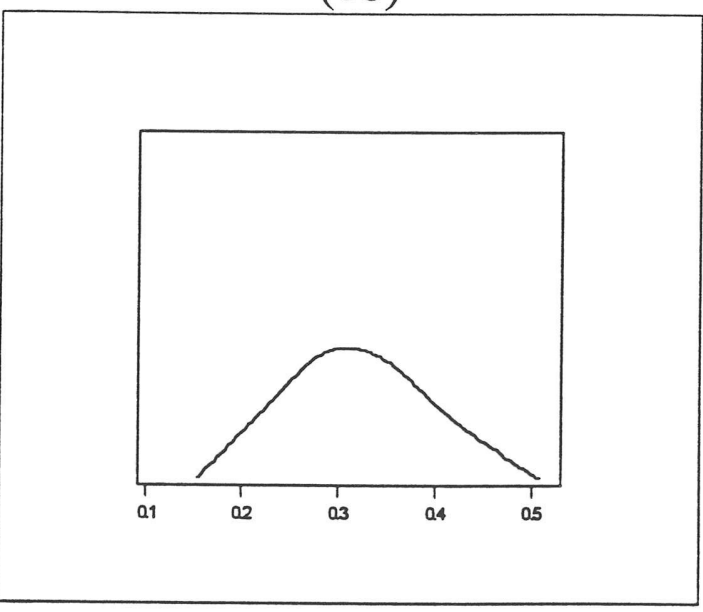

(6d)

Figura 6. Densidades a posteriori marginais de $\beta, \theta, k$ e $\alpha$, aproximadas pelo algoritmo amostrador de Gibbs e Metropolis-Hastings.

Na tabela (12), mostramos os sumários a posteriori dos parâmetros $\beta, \theta, k$ e $\alpha$, considerando as amostras geradas pelo algoritmo amostrador de Gibbs e Metropolis-Hastings. 
Tabela 12. Sumários a posteriori de $\beta, \theta, k$ e $\alpha$ obtidos pelo algoritmo amostrador de Gibbs com Metropolis, para o modelo de estatísticas de ordem gama generalizada.

\begin{tabular}{|c|lllc|}
\hline Parâmetros & Média & Mediana & Variância & $\begin{array}{c}\text { Intervalo de Credibilidade } \\
\text { Bayesiano 95\% }\end{array}$ \\
\hline$\theta$ & 30.3156 & 30.0851 & 13.1698 & $(24.0164 ; 38.0502)$ \\
$\alpha$ & 0.3121 & 0.3084 & 0.00390 & $(0.2090 ; 0.4412)$ \\
$\beta$ & 3.9708 & 3.8910 & 1.0353 & $(2.5434 ; 6.1262)$ \\
$k$ & 16.4026 & 16.0892 & 16.3394 & $(9.5141 ; 25.4061)$ \\
\hline
\end{tabular}

\section{Comparação das Estimativas Pontuais}

$\mathrm{Na}$ tabela (13), temos os estimadores de máxima verossimilhança e a média a posteriori aproximada por um estimador Monte Carlo de $\beta, \theta, k$ e $\alpha$.

Tabela 13. Estimadores de máxima verossimilhança e estimadores Monte Carlo da média a posteriori de $\beta, \theta, k$ e $\alpha$.

\begin{tabular}{|c|cc|}
\hline Parâmetros & \multicolumn{2}{|c|}{$\begin{array}{r}\text { Estimador Monte Carlo } \\
\text { aproximado pelo amostrador } \\
\text { de Gibbs com Metropolis }\end{array}$} \\
\hline$\theta$ & 29.4301 & 30.3156 \\
$\alpha$ & 0.27480 & 0.3121 \\
$\beta$ & 5.12000 & 3.9708 \\
$k$ & 18.2010 & 16.4026 \\
\hline
\end{tabular}

Observamos que as estimativas pontuais são razoavelmente próximas para os parâmetros $\beta, \theta, k$ e $\alpha$, considerando o método clássico e o método Bayesiano.

Sob o enfoque Bayesiano, as densidades a posteriori têm formas bem comportadas, validando, assim, os resultados obtidos aproximados. 


\section{Algoritmos Amostrador de Gibbs e Metropolis-Hastings}

para o Modelo de Estatísticas de Ordem Gama Generalizada Considerando a Inclusão da Variável Latente $\mathrm{N}^{\prime}=\mathrm{N}-\mathrm{n}$

Para a especificação e simplificação das densidades condicionais de $\beta, \theta, k$ e $\alpha$ do algoritmo amostrador de Gibbs e Metropolis-Hastings, consideramos a inclusão de uma variável latente, que se refere ao número de erros remanescentes no software.

Considerando os dados da tabela (11), as densidades a priori (4.2.9) obtemos as densidades condicionais de $\mathrm{N}^{\prime}, \beta, \theta, k \mathrm{e} \alpha$ dadas em (4.2.11), com $\mathrm{a}=60, \mathrm{~b}=2, \mathrm{c}=10, \mathrm{~d}=2, \mathrm{e}=12, \mathrm{f}=28, \mathrm{~g}=20 \mathrm{e} \mathrm{h}=1$. Assim, simulamos 10 seqüências de tamanho 2000 , com 10 valores iniciais arbitrários para cada variável $\mathrm{N}^{\prime}, \beta, \theta, k \mathrm{e} \alpha$.

Em cada seqüência, desprezamos os 500 pontos iniciais e selecionamos 100 pontos tomados de 15 em 15, resultando em uma amostra final de 1000 pontos amostrais.

A convergência do algoritmo amostrador de Gibbs com Metropolis foi verificada pelo critério de Gelman e Rubin (1992).

Os fatores de Redução de Escala para $\theta, \alpha, \beta$ e $k$ são dados por: $\mathrm{R}(\theta)=1.0039, \quad \mathrm{R}(\alpha)=1.0099, \quad \mathrm{R}(\beta)=1.0029, \quad \mathrm{R}(k)=1.0096 \quad \mathrm{e}$ $\mathrm{R}\left(\mathrm{N}^{\prime}\right)=0.9999$. Observe-se que $\mathrm{R}$ (fator de redução de escala), aproxima-se de 1. Portanto, é razoável considerarmos 20000 iterações para obter a convergência do algoritmo de Gibbs com Metropolis para esse modelo.

A taxa de aceitação do algoritmo de Metropolis-Hastings é $38.99 \%$ indicando uma razoável aceitação do algoritmo.

As densidades a posteriori marginais de $\mathrm{N}^{\prime}, \beta, \theta, k$ e $\alpha$, aproximadas pelo amostrador de Gibbs e Metropolis-Hastings, são apresentadas nas figuras (7e), (7a), (7b), (7c) e (7d), respectivamente. 


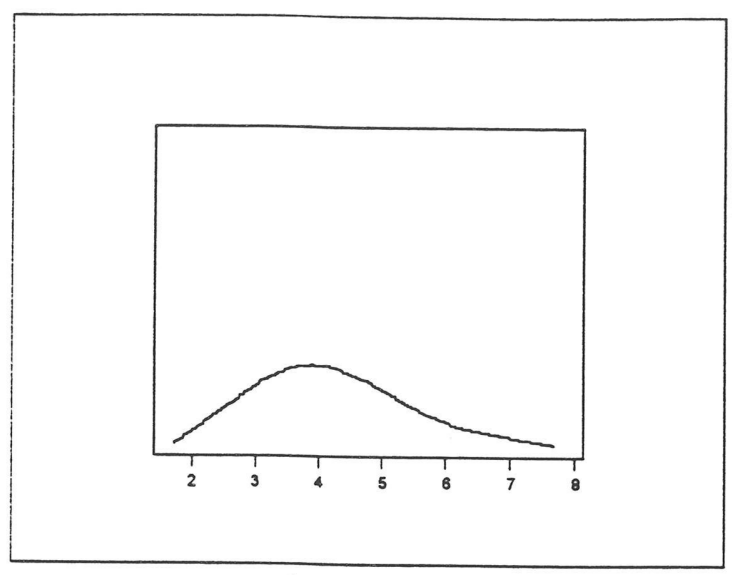

(7a)

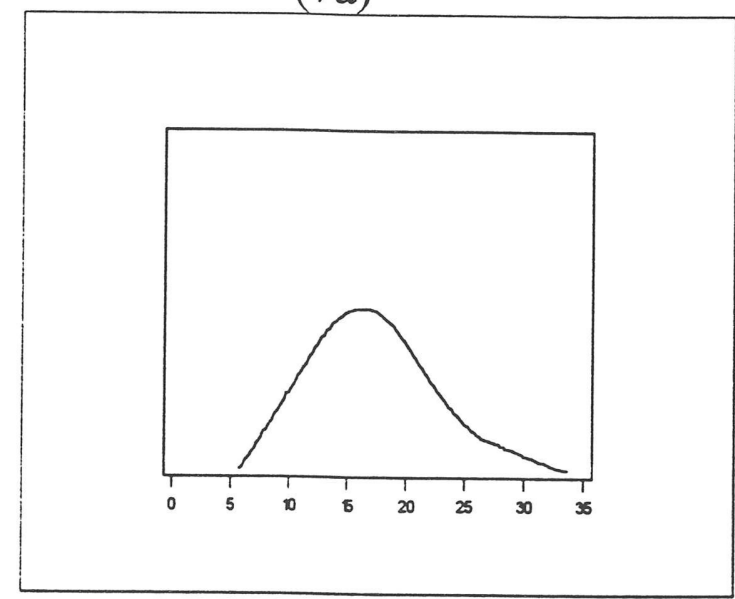

(7c)

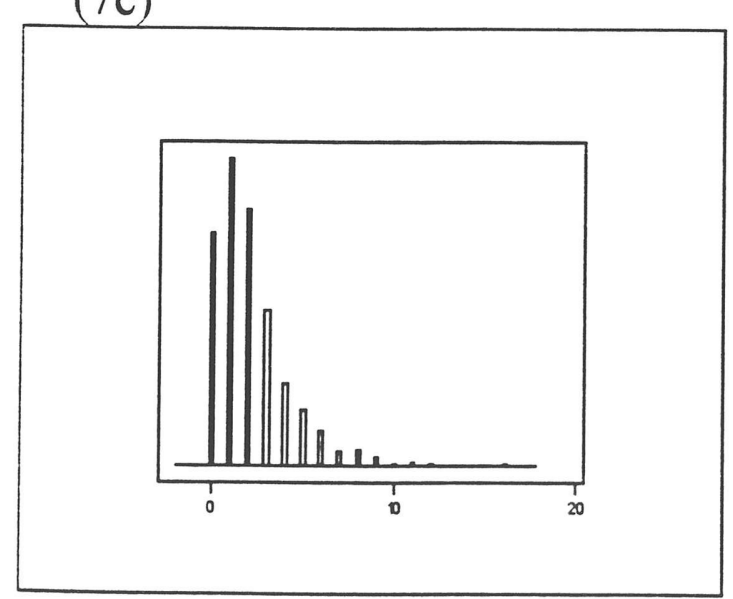

(7d)

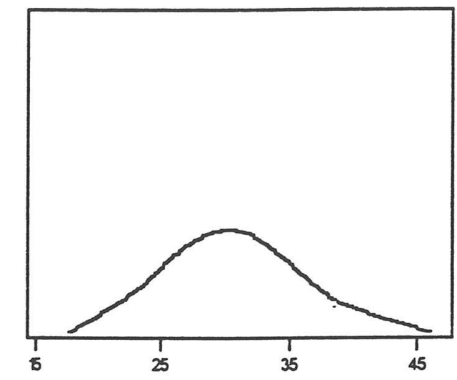

(7b)

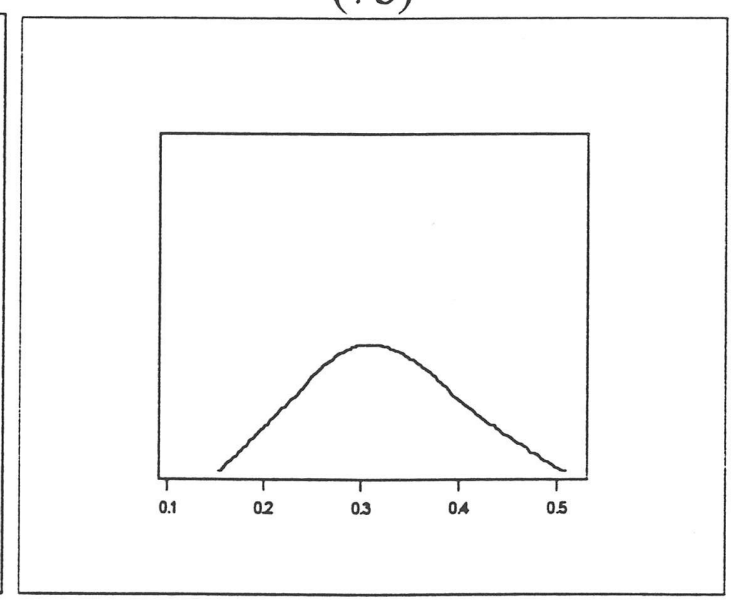

(7d)

Figura 7. Densidades a Posteriori Marginais de $\beta, \theta, k, \alpha$ e $\mathrm{N}^{\prime}$, respectivamente, obtidas pelo amostrador de Gibbs com Metropolis. 
Observamos, na figura (7), que as densidades a posteriori marginais de $\beta, \theta, k$ e $\alpha$ obtidas pelo algoritmo de Gibbs com Metropolis com inclusão da variável latente são similares às densidades a posteriori obtidas sem a inclusão da variável latente no algoritmo amostrador de Gibbs. Ver figura (6).

$\mathrm{Na}$ tabela (14) temos os sumários a posteriori dos parâmetros $\mathrm{N}^{\prime}$, $\beta, \theta, k$ e $\alpha$ considerando as amostras geradas pelo algoritmo amostrador de Gibbs com Metropolis.

Tabela 14. Sumários a posteriori de $\mathrm{N}^{\prime}, \beta, \theta, k$ e $\alpha$ obtidos pelo algoritmo amostrador de Gibbs com Metropolis incluindo a variável latente $\mathrm{N}^{\prime}$

\begin{tabular}{|c|llll|}
\hline Parâmetros & Média & Mediana & Variância & $\begin{array}{c}\text { Intervalo de Credibilidade } \\
\text { Bayesiano 95\% }\end{array}$ \\
\hline $\mathrm{N}^{\prime}$ & 2.4511 & 2.0000 & 1.0254 & $(1 ; 4)$ \\
$\theta$ & 29.996 & 30.010 & 12.1848 & $(24.0194 ; 38.1233)$ \\
$\alpha$ & 0.3061 & 0.3084 & 0.00390 & $(0.2101 ; 0.4316)$ \\
$\beta$ & 4.0125 & 3.9879 & 1.2455 & $(2.9874 ; 6.1257)$ \\
$k$ & 16.976 & 16.9201 & 16.4042 & $(9.1443 ; 25.0124)$ \\
\hline
\end{tabular}

Comparando os resumos a posteriori de $\beta, \theta, k$ e $\alpha$ obtidos pelo algoritmo de Gibbs sem a inclusão da variável latente (tabela, 12) com os resumos considerando a inclusão da variável latente (tabela, 14), notamos resultados bastante semelhantes.

\section{Comparação da Convergência}

A tabela (15) apresenta o fator de redução de escala do critério de Gelman e Rubin (1992) considerados para verificar a convergência do amostrador de Gibbs com Metropolis sem a inclusão da variável latente e com a inclusão da variável latente para os parâmetros $\beta, \theta, k$ e $\alpha$. 
Tabela 15. Fator de redução de escala do critério de Gelman e Rubin (1992) para os algoritmos amostrador de Gibbs e Metropolis Hastings sem a inclusão da variável latente e considerando a variável latente.

\begin{tabular}{|c|c|c|}
\hline Parâmetros & $\begin{array}{l}\text { Fator de Redução de Escala } \\
\text { (com inclusão da variável } \mathrm{N}^{\prime}\end{array}$ & $\begin{array}{l}\text { Fator de Redução de Escala } \\
\quad\left(\text { sem a variável } \mathrm{N}^{\prime}\right)\end{array}$ \\
\hline$\beta$ & 1.0029 & 1.0178 \\
\hline$\alpha$ & 1.0099 & 1.0099 \\
\hline$\theta$ & 1.0039 & 0.9998 \\
\hline$k$ & 1.0096 & 1.0048 \\
\hline
\end{tabular}

Observamos na tabela (15), que a inclusão da variável latente $\mathrm{N}^{\prime}$, melhora a convergência do algoritmo amostrador de Gibbs e MetropolisHastings e, como comentamos no Capítulo 4, auxilia a especificação e simplificação das densidades condicionais dos parâmetros envolvidos.

Usualmente, engenheiros de software consideram casos especiais do modelo com função de valor médio tipo gama generalizada (6.2.1) para analisar os dados de confiabilidade de software. Por exemplo quando $k=1$ em (6.2.1), temos o processo de Goel (1983), se $\alpha=1$ em (6.2.1) temos o modelo de confiabilidade de software Tipo Gama e quando $k=1$ e $\alpha=1$ em (6.2.1) temos o processo de Goel e Okumoto (1979).

A seguir, analisamos os modelos de Goel (1983), Goel e Okumoto (1979) e modelo Tipo Gama, utilizando métodos Bayesianos e o algoritmo amostrador de Gibbs e Metropolis-Hastings.

\section{Análise Bayesiana do Modelo de Goel (1983) Assumindo $k=1$}

Assumindo $k=1$ em (6.2.1), temos o modelo de estatísticas de ordem Weibul, com função de valor médio dada por,

$$
m(t)=\theta\left(1-e^{-\beta t^{\alpha}}\right)
$$


onde $\theta>0, \beta>0$ e $\alpha>0$.

Considerando-se o modelo de Goel (1983) com parâmetros $\theta, \alpha$ e $\beta$ e os dados da tabela (11), aproximamos as densidades a posteriori marginais de $\theta, \alpha$ e $\beta$ utilizando-se o amostrador de Gibbs com Metropolis.

Consideramos a inclusão da variável latente $\mathrm{N}^{\prime}$, para algoritmo amostrador de Gibbs. Assim, obtemos as densidades condicionais de $\mathrm{N}^{\prime}, \theta, \alpha$ e $\beta$ (4.2.16), com $\mathrm{a}=60, \mathrm{~b}=2, \mathrm{c}=10, \mathrm{~d}=2, \mathrm{e}=3.6$ e $\mathrm{f}=7.2$.

Utilizando-se o amostrador de Gibbs e Metropolis-Hastings simulamos 10 cadeias com 1200 pontos amostrais, considerando 10 valores iniciais arbitrários de $\mathrm{N}^{\prime}, \theta, \alpha$ e $\beta$. Em cada seqüência, desprezamos os 200 valores inicias e dos 1000 pontos restantes selecionamos 100 pontos escolhidos de de 10 em 10, obtendo assim, uma amostra final de 1000 pontos.

Os fatores de Redução de Escala do critério de Gelman e Rubin (1992) para os parâmetros $N^{\prime}, \theta, \alpha$ e $\beta$ são dados por: $R(\theta)=1.0076$, $\mathrm{R}(\alpha)=1.009, \mathrm{R}(\beta)=1.0055$ e $\mathrm{R}\left(\mathrm{N}^{\prime}\right)=1.018$. A taxa de aceitação desse modelo para o algoritmo de Metropolis Hastings é dada por $41.02 \%$.

Podemos observar que o fator de Redução de Escala aproxima-se de 1 para todos os parâmetros, assim é razoável considerar 12000 iterações para a convergência do algoritmo amostrador de Gibbs com Metropolis para o modelo de Goel (1983).

$\mathrm{Na}$ figura (8), temos as densidades a posteriori marginais dos parâmetros $\mathrm{N}^{\prime}, \theta, \alpha$ e $\beta$, aproximadas pelo amostrador de Gibbs e Metropolis-Hastings. 

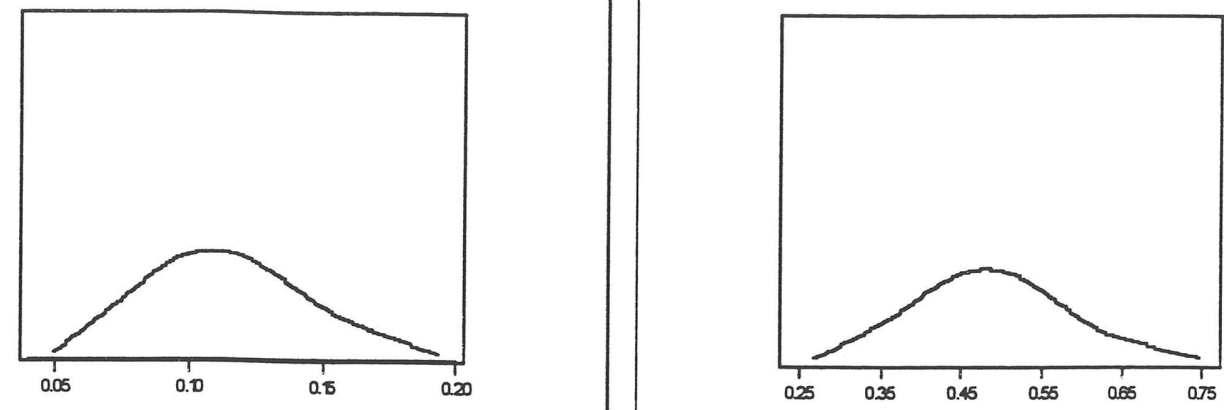

(8a)
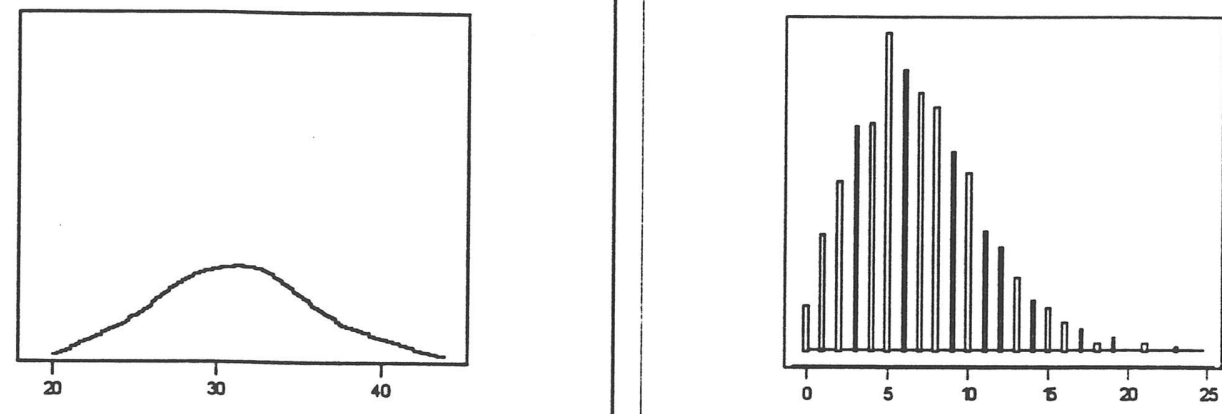

(8c)

(8d)

Figura 8. Densidades a posteriori marginais de $\beta, \alpha, \theta$ e $\mathrm{N}^{\prime}$, dadas em (8a), (8b), (8c) e (8d), respectivamente, aproximadas pelo amostrador de Gibbs e Metropolis-Hastings.

Na tabela (16), temos os sumários a posteriori dos parâmetros $\mathrm{N}^{\prime}, \theta, \alpha$ e $\beta$, obtidos com a utilização das amostras geradas pelo amostrador de Gibbs e Metropolis-Hastings. 
Tabela 16. Sumários a posteriori de $\mathrm{N}^{\prime}, \theta, \alpha$ e $\beta$ obtidos pelo amostrador de Gibbs e Metropolis-Hastings, para o modelo de estatísticas de ordem Weibul.

\begin{tabular}{|c|llll|}
\hline Parâmetros & Média & Mediana & Variância & $\begin{array}{c}\text { Intervalo de Credibilidade } \\
\text { Bayesiano 95\% }\end{array}$ \\
\hline $\mathrm{N}^{\prime}$ & 6.949 & 6.0000 & 9.879 & $(1 ; 16)$ \\
$\theta$ & 30.854 & 30.684 & 5.2542 & $(25.1755 ; 37.2316)$ \\
$\alpha$ & 0.3061 & 0.3084 & 0.00390 & $(0.0 .3573 ; 0.6107)$ \\
$\beta$ & 0.11148 & 0.11010 & 0.04497 & $(0.0724 ; 0.1609)$ \\
\hline
\end{tabular}

\section{Análise Bayesiana do Modelo de Confiabilidade de Softwate Tipo}

\section{Gama Assumindo $\alpha=1$}

Assumindo $\alpha=1$ em (6.2.1), temos o modelo de estatísticas de ordem gama, com função de valor médio dada por,

$$
\mathrm{m}(\mathrm{t})=\theta\left(1-\mathrm{e}^{-\beta \mathrm{t}} \sum_{\mathrm{j}=0}^{k-1} \frac{(\beta \mathrm{t})^{\mathrm{j}-1}}{\mathrm{j} !}\right)
$$

onde $k>0$ e $\beta>0$.

Considerando-se o modelo de confiabilidade de software tipo Gama e os dados da tabela (11), encontramos os estimadores de máxima verossimilhança de $\theta, k$ e $\beta$ dados por $\hat{\theta}=29.5, \hat{k}=1.884$ e $\hat{\beta}=0.01665$ (obtidos pelo método de Newton).

Utilizando-se a informação dos dados, através dos estimadores de máxima verossimilhança de $\mathrm{N}^{\prime}, \theta, k$ e $\beta$, obtemos as densidades condicionais descritas em (4.2.13), com a=90, b=3, c=12, d=680, e=9.0 e $\mathrm{f}=4.75$.

Usando o amostrador de Gibbs e Metropolis-Hastings, simulamos 10 cadeias de tamanho 1200, considerando-se 10 valores iniciais arbitrários de $N^{\prime}, \theta, \alpha$ e $\beta$. Em cada seqüência, desprezamos os 200 valores inicias e dos 
1000 pontos restantes selecionamos 100 , escolhidos de de 10 em 10 pontos, obtendo, assim, uma amostra final de 1000 pontos.

Os fatores de Redução de Escala do critério de Gelman e Rubin (1992) para os parâmetros $\mathrm{N}^{\prime}, \theta, k$ e $\beta$ são dados por: $\mathrm{R}(\theta)=1.0026$, $\mathrm{R}(k)=1.0091, \mathrm{R}(\beta)=1.0132$ e $\mathrm{R}\left(\mathrm{N}^{\prime}\right)=1.0096$.

A taxa de aceitação para o algoritmo de Metropolis Hastings é $41.40 \%$, indicando uma razoável aceitação desse algoritmo para o modelo de estatísticas de ordem gama.

Podemos observar que os fatores de Redução de Escala aproximamse de 1 para todos os parâmetros. Assim, é razoável considerar 12000 iterações para a convergência desse algoritmo.

A figura (9), mostra as densidades a posteriori marginais de $\beta, \theta, \mathrm{N}^{\prime} \mathrm{e} \mathrm{k}$, aproximadas pelo algoritmo amostrador de Gibbs e Metropolis-Hastings, ver figuras (9a), (9b), (9c) e (9d), respectivamente.

A tabela (17), apresenta os sumários a posteriori para $\beta, \theta, \mathrm{N}^{\prime} \mathrm{e} \mathrm{k}$, obtidos usando o amostrador de Gibbs e Metropolis-Hastings. 


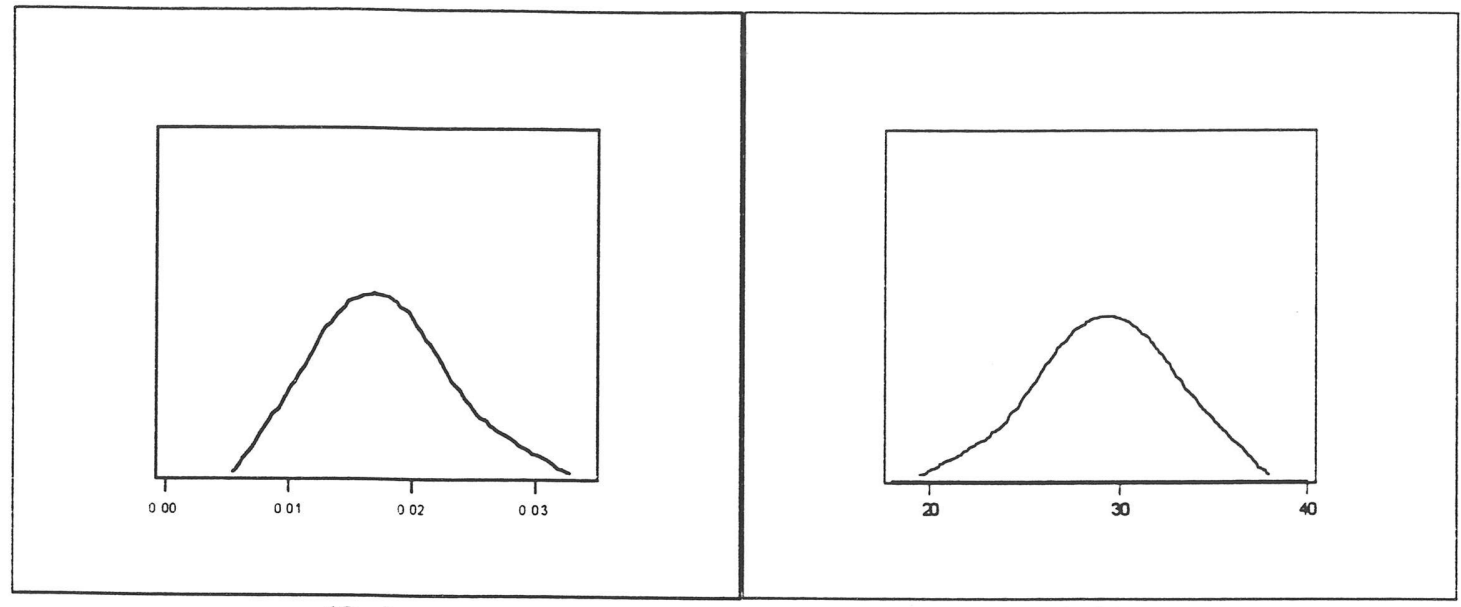

(9a)

(9b)

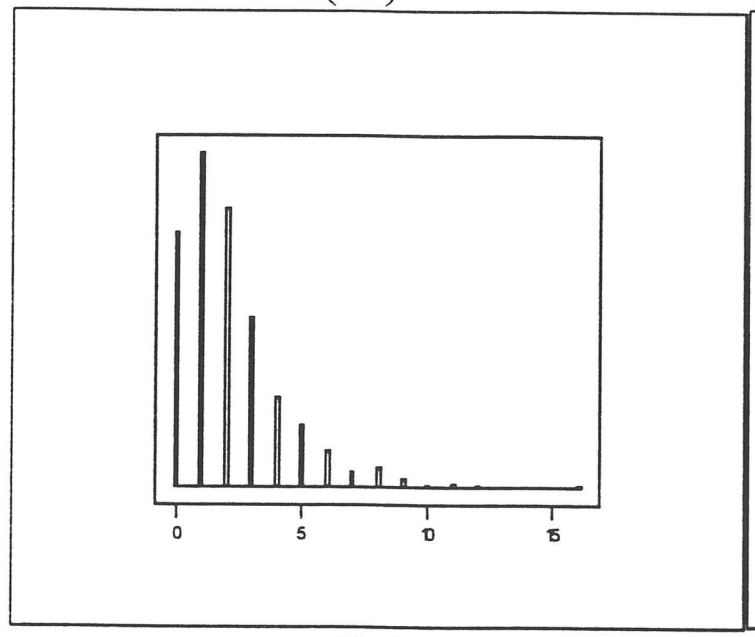

(9c)

$(9 \mathrm{~d})$

Figura 9. Densidades a Posteriori Marginal de $\beta, \theta, N^{\prime}$ e $k$, respectivamente, obtidas pelo amostrador de Gibbs com Metropolis.

Tabela 17. Sumários a Posteriori de $\beta, \theta, N^{\prime}$ e $k$ obtidos pelo amostrador de Gibbs com Metropolis, para o modelo de estatísticas de ordem gama.

\begin{tabular}{|c|llll|}
\hline Parâmetros & Média & Mediana & Variância & $\begin{array}{c}\text { Intervalo de Credibilidade } \\
\text { Bayesiano 95\% }\end{array}$ \\
\hline $\mathrm{N}^{\prime}$ & 2.1310 & 2.000 & 4.1360 & $(0 ; 8)$ \\
$\theta$ & 29.4676 & 29.3576 & 7.914500 & $(24.2259 ; 35.5030)$ \\
$k$ & 1.8750 & 1.8364 & 0.1491 & $(1.9997 ; 2.7292)$ \\
$\beta$ & 0.0171 & 0.0168 & 0.00184 & $(0.0098 ; 0.0266)$ \\
\hline
\end{tabular}




\section{Análise Bayesiana do Modelo de Goel e Okumoto (1979)}

Assumindo $k=1$ e $\alpha=1$ em (6.2.1) temos o processo de Goel e Okumoto (1979), com função de valor médio dada por,

$$
m(t)=\theta\left(1-e^{-\beta t}\right)
$$

onde $\theta>0$ e $\beta>0$.

Para inferir sobre os parâmetros $\theta$ e $\beta$, aproximamos as densidades a posteriori marginais utilizando o amostrador de Gibbs e MetropolisHastings.

Utilizando os dados da tabela (11), os estimadores de máxima verossimilhança de $\mathrm{N}^{\prime}, \theta$ e $\beta$ obtemos as densidades condicionais de $\mathrm{N}^{\prime}, \theta$ e $\beta$ dadas em (4.2.14), com $\mathrm{a}=60, \mathrm{~b}=2, \mathrm{c}=5, \mathrm{~d}=1000$.

Assim, geramos, pelo amostrador de Gibbs 10 seqüências de 1200 pontos amostrais. Em cada seqüência, desprezamos os 200 valores iniciais e selecionamos 100 pontos tomados de $10 \mathrm{em} \mathrm{10}$, resultando assim em uma amostra final de 1000 pontos amostrais.

Os fatores de Redução de Escala do critério de Gelman e Rubin (1992) são dados por: $R(\beta)=1.001, R(\theta)=1.000$ e $R\left(N^{\prime}\right)=0.9999$. Observe que os fatores de Redução de Escala aproximam-se de 1 para todos os parâmetros. Portanto, é razoável considerar-se 12000 iterações para obter convergência em distribuição para esse modelo.

A figura (10) mostra as densidades a posteriori de $\mathrm{N}^{\prime}, \theta$ e $\beta$ aproximadas pelo algoritmo amostrador de Gibbs. 

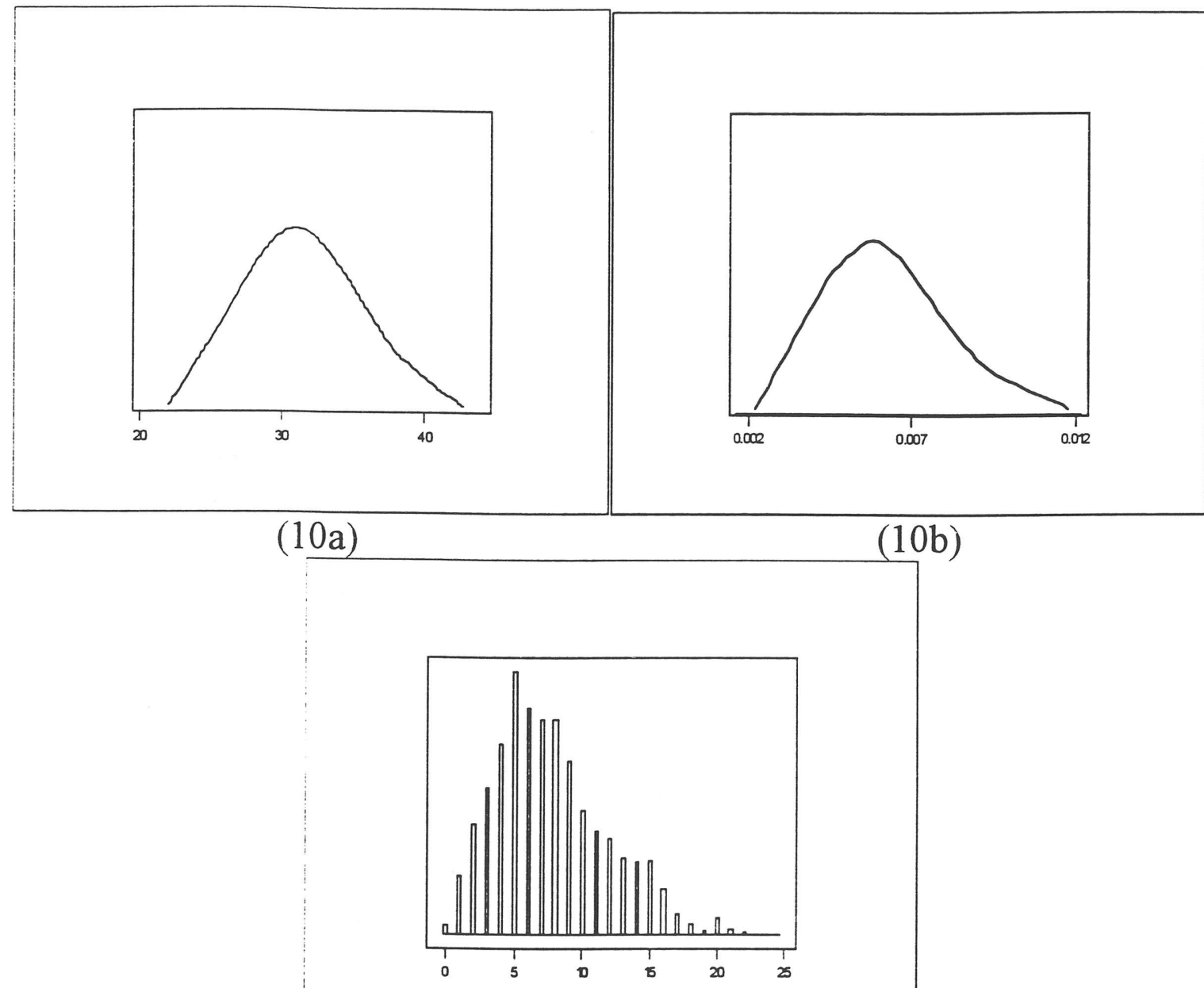

(10c)

Figura 10. Densidades a Posteriori Marginais de $\theta, \beta$ e $N^{\prime}$, respectivamente, obtidas pelo amostrador de Gibbs com Metropolis.

Na tabela (18) temos os sumários a posteriori de $\theta, \beta$ e $\mathrm{N}^{\prime}$.

Tabela 18. Sumários a posteriori $\operatorname{de} \theta, \beta$ e $\mathrm{N}^{\prime}$, obtidos pelo amostrador de Gibbs e Metropolis-Hastings, para o modelo de Goel e Okumoto (1979).

\begin{tabular}{|c|lccc|}
\hline Parâmetros & Média & Mediana & Variância & $\begin{array}{c}\text { Intervalo de Credibilidade } \\
\text { Bayesiano 95\% }\end{array}$ \\
\hline $\mathrm{N}^{\prime}$ & 7.724 & 7.000 & 17.2070 & $(1 ; 17)$ \\
$\theta$ & 31.2174 & 31.0882 & 12.4283 & $(24.8528 ; 38.1791)$ \\
$\beta$ & 0.0059 & 0.0058 & $2,428 \times 10^{-6}$ & $(0.0034 ; 0.0094)$ \\
\hline
\end{tabular}




\section{Análise Bayesiana do Modelo de Estatísticas de Ordem Log-Normal}

Admitindo-se o modelo de estatísticas de ordem log-normal com função de valor médio descrita em (4.1.12), para modelar os dados da tabela (11), temos interesse em inferir dos parâmetros $\theta, \mu$ e $\sigma$ ou função dos mesmos.

Para aproximar as densidades a posteriori marginais de $\theta, \mu \mathrm{e} \sigma$, usamos o amostrador de Gibbs e Metropolis-Hastings com inclusão de uma variável latente $\mathrm{N}^{\prime}=\mathrm{N}-26$ (número de erros remanescentes no software).

Utilizando-se a informação que os dados fornecem a respeito de $\theta, \mu$ e $\sigma$, através dos estimadores de máxima verossimilhança $\hat{\mu}=4.55$, $\hat{\sigma}=0.84$ e $\hat{\theta}=30$, obtemos as contantes das densidades a priori de $N^{\prime}$ e $\theta$ definidas em (4.2.17), assim temos $\mathrm{N}^{\prime} \sim \mathrm{P}\left[\theta-\Phi\left(\frac{\ln 250-\mu}{\sigma^{2}}\right)\right]$ e $\theta \sim \Gamma(36,1.2)$, as quais são utilizadas para gerar os parâmetros $N^{\prime}$ e $\theta$. Para encontrar os núcleos da cadeia de Markov, utilizados para gerar candidatos dos parâmetros $\mu$ e $\sigma$, multiplicamos e dividimos as densidades condicionais de $\mu$ e $\sigma$, definidas em (4.2.17), pelas densidades $\mu \sim \mathrm{N}(4.5,2.0)$ e $\sigma \sim \Gamma(8.11,9.5)$. Assim, simulamos 10 cadeias de tamanho 1200, considerando-se 10 valores iniciais arbitrários de $N^{\prime}, \theta, \mu$ e $\sigma$. Em cada seqüência, desprezamos os 200 valores inicias e dos 1000 pontos restantes selecionamos 100 pontos escolhidos de 10 em 10, obtendo, assim, uma amostra final de 1000 pontos.

Os fatores de Redução de Escala do critério de Gelman e Rubin (1992) para os parâmetros $\mathrm{N}^{\prime}, \theta, \mu$ e $\sigma$ são dados por: $\mathrm{R}(\theta)=1.010$, $\mathrm{R}(\mu)=1.0091, \mathrm{R}(\lambda)=1.002$ e $\mathrm{R}\left(\mathrm{N}^{\prime}\right)=1.0099$. A taxa de aceitação desse modelo para o algoritmo de Metropolis-Hastings é dada por 39.01\%, indicando uma aceitação razoável desse algoritmo.

Podemos observar que o fator de redução de escala aproxima-se de 1 para todos os parâmetros. Assim é razoável considerar 12000 iterações para 
a convergência do algoritmo amostrador de Gibbs e Metropolis-Hastings para o modelo de estatísticas de ordem log normal.

A figura (11) apresenta as densidades a posteriori marginais de $\mathrm{N}^{\prime}, \theta, \mu$ e $\sigma$, obtidas pelo amostrador de Gibbs e Metropolis-Hastings. Ver figuras (11c), (11a), (11d) e (11b), respectivamente.

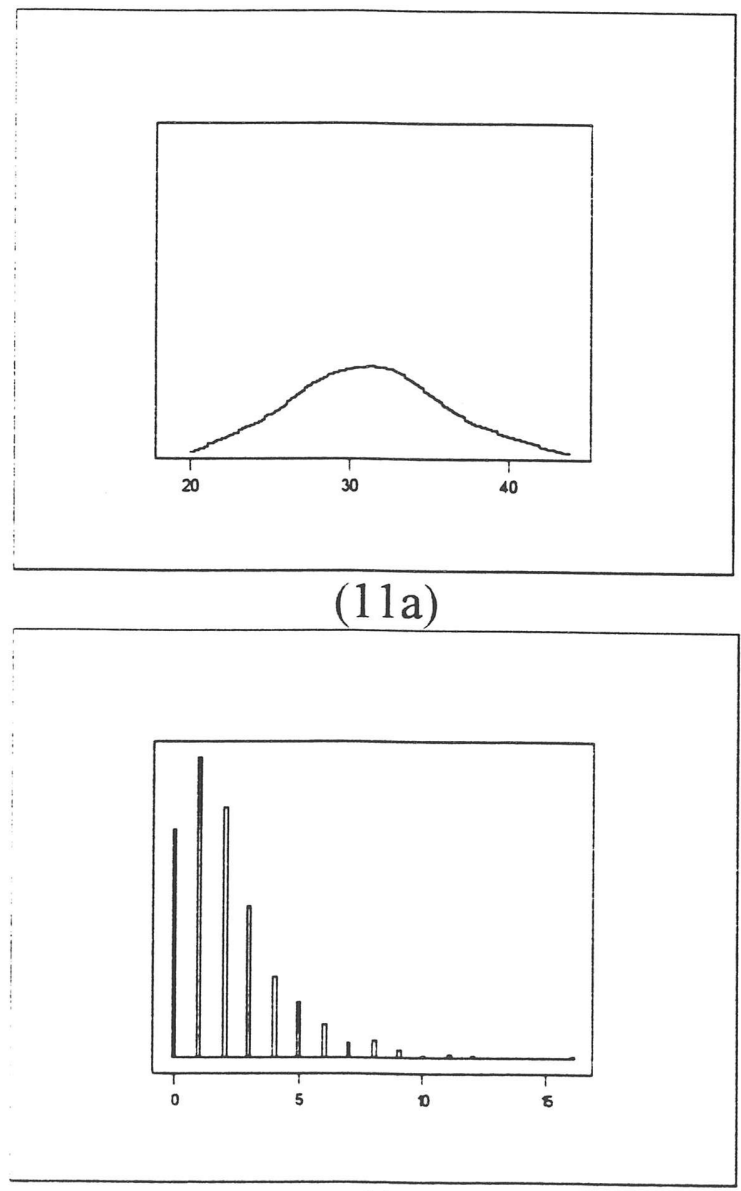

(11c)

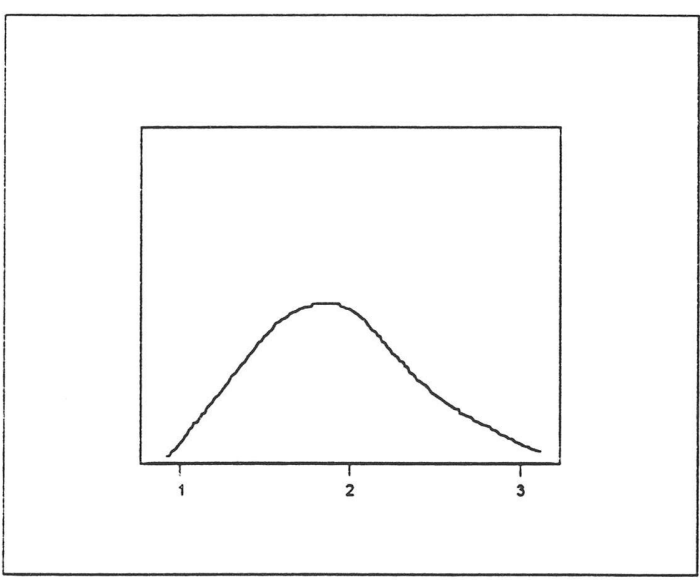

(11b)

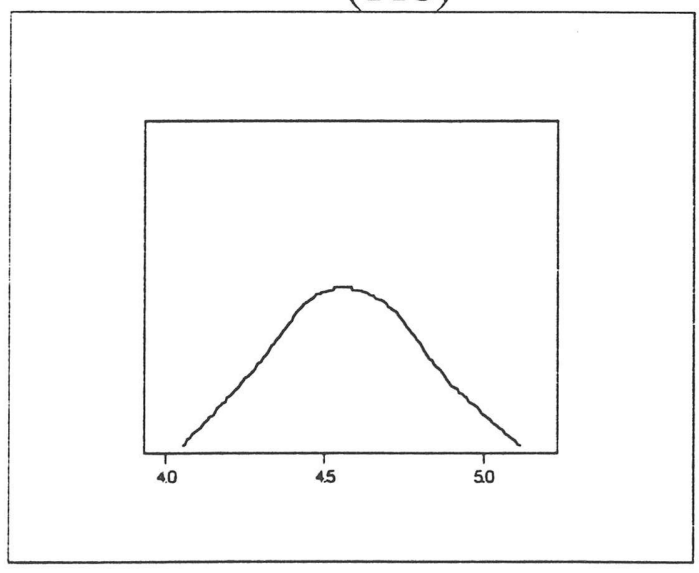

(11d)

Figura 11. Densidades a posteriori de $\theta, \sigma, \mathrm{N}^{\prime}$ e $\mu$, obtidas pelo amostrador de Gibbs e Metropolis-Hastings.

$\mathrm{Na}$ tabela (19) temos os sumários a posteriori de $\theta, \sigma, \mathrm{N}^{\prime}$ e $\mu$, obtidos considerando-se as amostras geradas pelo amostrador de Gibbs e Metropolis-Hastings. 
Tabela 19. Sumários a posteriori de $\theta, \sigma, N^{\prime}$ e $\mu$, obtidos pelo amostrador de Gibbs e Metropolis-Hastings, para o modelo de estatísticas de ordem log-normal.

\begin{tabular}{|c|llll|}
\hline Parâmetros & Média & Mediana & Variância & $\begin{array}{c}\text { Intervalo de Credibilidade } \\
\text { Bayesiano 95\% }\end{array}$ \\
\hline $\mathrm{N}^{\prime}$ & 4.2001 & 2.000 & 24.812 & $(0 ; 21)$ \\
$\theta$ & 30.105 & 29.844 & 16.451 & $(22.511 ; 40.173)$ \\
$\mu$ & 4.59 & 4.58 & 0.1706 & $(4.13 ; 5.94)$ \\
$\sigma$ & 0.854 & 0.7787 & 0.449 & $(0.567 ; 1.7012)$ \\
\hline
\end{tabular}

\section{Análise Bayesiana do Modelo de Estatísticas de Ordem Gaussiana Inversa}

Admitamos o modelo de falha truncado com $t$ substituído por $\mathrm{x}_{26}=250$, e o modelo de estatísticas de ordem gaussiana inversa para modelar os dados da tabela (11), com função de valor médio dada por

$$
\mathrm{m}(\mathrm{t})=\theta\left\{\Phi\left[\sqrt{\frac{\lambda}{250}}\left(\frac{250}{\mu}-1\right)\right]+\mathrm{e}^{2 \lambda / \mu} \Phi\left[-\sqrt{\frac{\lambda}{250}}\left(1+\frac{250}{\mu}\right)\right]\right\}
$$

onde $\theta>0, \mu>0$ e $\lambda>0$.

Tomando-se os dados da tabela (11) e os estimadores de máxima verossimilhança $\hat{\mu}=275.3, \hat{\lambda}=100$ e $\hat{\theta}=35.6$ (obtidos pelo método de Newton), encontramos os valores das constantes $\mathrm{a}_{2}, \mathrm{~b}_{2}, \mathrm{a}_{3}, \mathrm{~b}_{3}, \mathrm{a}_{4}$ e $\mathrm{b}_{4}$ das densidades a priori definidas em (4.2.19), obtendo-se assim as densidades a priori $\quad \mu \sim \Gamma(756 ; 2.75), \quad \lambda \sim \Gamma(100 ; 1), \quad \theta \sim \Gamma(81 ; 2.25) \quad$ e $\mathrm{N}^{\prime} \sim \mathrm{P}(\theta(1-\mathrm{F}(\mathrm{t}))$. Utilizando essas densidades, geramos, pelo amostrador de Gibbs e Metropolis-Hastings, 10 cadeias com 1200 pontos amostrais cada. Em cada seqüência, desprezamos os 200 valores iniciais e selecionamos 100 pontos tomados de 10 em 10, obtendo uma amostra final de 1000 pontos amostrais. 
Os fatores de Redução de Escala para os parâmetros $\theta, \sigma, \mathrm{N}^{\prime}$ e $\mu$ são dado por, $R(\theta)=1.000, R(\mu)=1.001, R(\sigma)=1.0006$ e $R\left(N^{\prime}\right)=0.999$. A taxa de aceitação desse algoritmo é de $44.1 \%$. Podemos observar que a taxa de aceitação e a convergência em distribuição desse algoritmo são bastante satisfatórias.

$\mathrm{Na}$ tabela (20), temos os sumários a posteriori dos parâmetros $\theta, \sigma, \mathrm{N}^{\prime}$ e $\mu$, e na figura (12), mostramos as densidades a posteriori marginais de $\theta, \sigma, \mathrm{N}^{\prime}$ e $\mu$, obtidas utilizando a amostra ( $\left.\mathrm{S}=1000\right)$ gerada pelos algoritmos amostrador de Gibbs e Metropolis-Hastings.

Tabela 20. Sumários a posteriori de $\theta, \sigma, \mathrm{N}^{\prime}$ e $\mu$, obtidos pelo amostrador de Gibbs e Metropolis-Hastings, para o modelo de estatísticas de ordem gaussiana inversa.

\begin{tabular}{|c|lllc|}
\hline Parâmetros & Média & Mediana & Variância & $\begin{array}{c}\text { Intervalo de Credibilidade } \\
\text { Bayesiano 95\% }\end{array}$ \\
\hline $\mathrm{N}^{\prime}$ & 10.4270 & 10 & 11.3821 & $(5.0,18.0)$ \\
$\theta$ & 36.2004 & 36.1753 & 11.6491 & $(29.8630 ; 43.4157)$ \\
$\mu$ & 274.6688 & 274.6903 & 87.7225 & $(256.012 ; 293.322)$ \\
$\sigma$ & 100.1174 & 99.9262 & 84.8426 & $(82.971 ; 119.424)$ \\
\hline
\end{tabular}



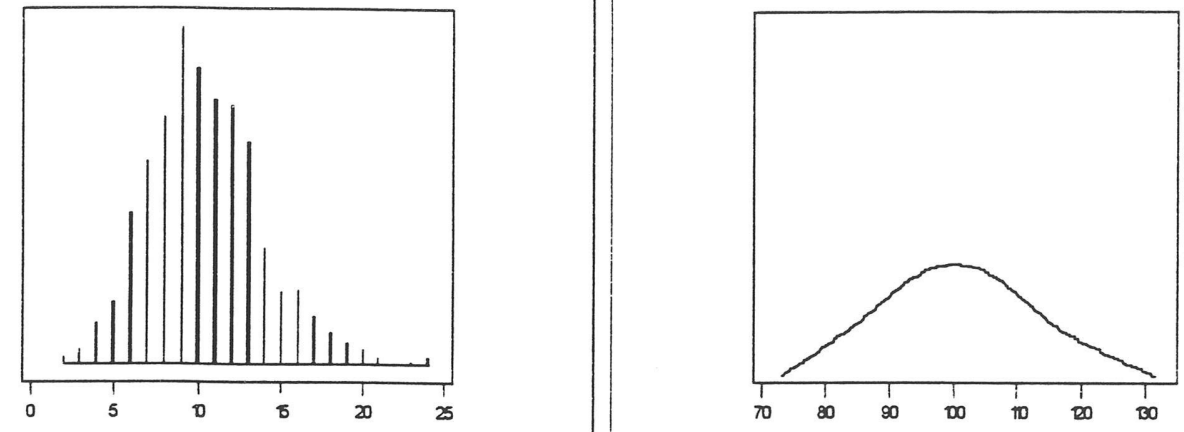

(12a)
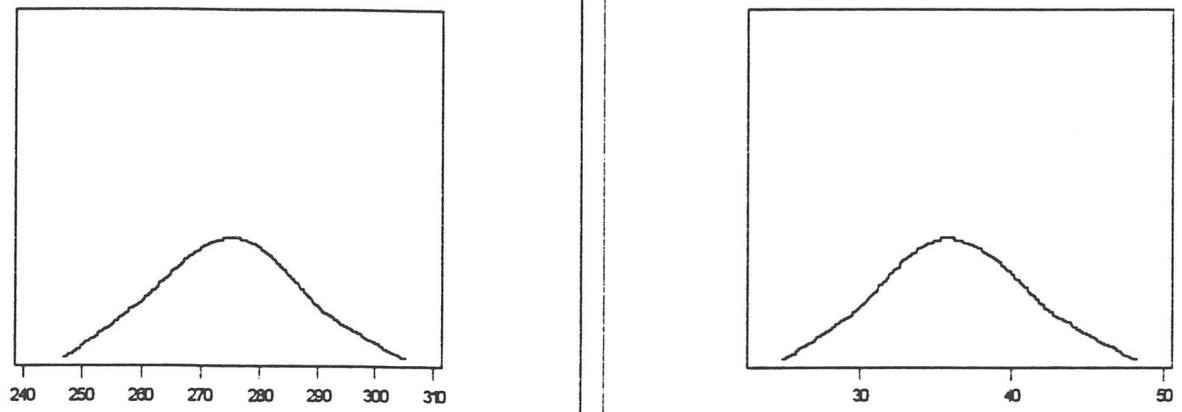

$(12 \mathrm{c})$

$(12 \mathrm{~d})$

Figura 12. Densidades a posteriori de $\mathrm{N}^{\prime}, \lambda, \mu$ e $\theta$, (12a), (12b), (12c) e (12d), respectivamente obtidas pelo amostrador de Gibbs e Metropolis-Hastings.

\section{Discriminação Bayesiana dos Modelos de Estatísticas de Ordem}

A seguir, conduziremos a discriminação Bayesiana dos modelos de estatísticas de ordem gama, gama-generalizada, log-normal, exponencial, Weibul e gaussiana inversa, utilizando as técnicas de seleção de modelos apresentadas no capítulo 5 .

$\mathrm{Na}$ tabela (21), temos os estimadores Monte Carlo das funções de valor médio (4.1.5), (4.1.7), (4.1.8), (4.1.10), (4.1.12) e (4.1.14) com 
respeito à função de perdâ quadrática, utilizando os dados da tabela (11) e as amostras geradas pelo amostrador de Gibbs e Metropolis-Hastings.

Tabela 21. Estimadores Monte Carlo das funções de valor médio $m\left(x_{i}\right)$ dos modelos de estatísticas de ordem gama generalizada, Weibul, gama, exponencial, lognormal e gaussiana inversa.

\begin{tabular}{|c|c|c|l|c|l|l|l|l|}
\hline $\mathrm{i}$ & $\mathrm{xi}$ & $\mathrm{n}_{\mathrm{i}}$ & $\begin{array}{l}\text { Gama } \\
\text { Generalizada }\end{array}$ & Weibul & Gama & Exponencial & $\begin{array}{c}\text { Log- } \\
\text { Normal }\end{array}$ & $\begin{array}{l}\text { gaussiana } \\
\text { inversa }\end{array}$ \\
\hline 1 & 9 & 1 & 0.3847 & 8.4412 & 0.5877 & 1.6011 & 0.1416 & 0.0442 \\
2 & 21 & 2 & 2.0812 & 11.7309 & 2.1222 & 3.4021 & 1.1853 & 1.4955 \\
3 & 32 & 3 & 4.3854 & 13.6602 & 3.9554 & 5.3110 & 3.0754 & 3.9496 \\
4 & 36 & 4 & 5.3102 & 14.2111 & 4.6694 & 5.8544 & 3.8987 & 4.8902 \\
5 & 43 & 5 & 6.8978 & 15.1044 & 5.9728 & 6.8610 & 5.4101 & 6.4974 \\
6 & 45 & 6 & 7.3999 & 15.3388 & 6.3544 & 7.1546 & 5.8612 & 6.9412 \\
7 & 50 & 7 & 8.6423 & 15.8751 & 7.3012 & 7.8012 & 6.8777 & 8.0144 \\
8 & 58 & 8 & 10.4689 & 16.6410 & 8.8199 & 8.8614 & 8.8012 & 9.6159 \\
9 & 63 & 9 & 11.5575 & 17.0676 & 9.7751 & 9.4755 & 9.9122 & 10.5444 \\
10 & 70 & 10 & 13.0123 & 17.6201 & 11.064 & 10.3319 & 11.255 & 11.7552 \\
11 & 71 & 11 & 13.2117 & 17.6944 & 11.243 & 10.4421 & 11.4512 & 11.9201 \\
12 & 77 & 12 & 14.3755 & 18.1321 & 12.441 & 11.1318 & 12.5914 & 12.8696 \\
13 & 78 & 13 & 14.5507 & 18.1914 & 12.495 & 11.2449 & 12.787 & 13.0214 \\
14 & 87 & 14 & 16.1164 & 18.7677 & 14.005 & 12.2513 & 14.3522 & 14.3118 \\
15 & 91 & 15 & 16.7584 & 18.9998 & 14.559 & 12.7010 & 14.9978 & 14.9442 \\
16 & 92 & 16 & 16.9130 & 19.0541 & 14.883 & 12.7431 & 15.1311 & 14.9737 \\
17 & 95 & 17 & 17.3422 & 19.2331 & 15.335 & 13.0947 & 15.6012 & 15.3535 \\
18 & 98 & 18 & 17.8001 & 19.4011 & 15.778 & 13.4119 & 16.0411 & 15.7212 \\
19 & 104 & 19 & 18.6199 & 19.7107 & 16.744 & 13.8754 & 16.8841 & 16.4220 \\
20 & 105 & 20 & 18.7422 & 19.7644 & 16.754 & 14.0844 & 17.0079 & 16.5346 \\
21 & 116 & 21 & 20.0765 & 22.2999 & 18.288 & 15.1004 & 18.3666 & 17.6994 \\
22 & 149 & 22 & 22.9722 & 21.5820 & 21.956 & 17.8999 & 21.4785 & 20.5412 \\
23 & 156 & 23 & 23.4415 & 21.8456 & 22.601 & 18.2606 & 21.9803 & 21.0447 \\
24 & 247 & 24 & 26.7045 & 24.0511 & 27.433 & 23.2911 & 25.8897 & 25.6768 \\
25 & 249 & 25 & 26.8011 & 24.0877 & 27.511 & 23.4143 & 25.9214 & 25.7507 \\
26 & 250 & 26 & 26.7644 & 24.1055 & 27.601 & 23.3999 & 25.9561 & 25.7873 \\
\hline
\end{tabular}

Podemos observar que as estimativas Bayesianas da função de valor médio para os modelos de estatísticas de ordem gama, gama-generalizada, log-normal, exponencial e gaussiana inversa são próximas. Notamos uma razoável discrepância das estimativas Bayesianas $\hat{\mathrm{m}}\left(\mathrm{x}_{\mathrm{i}}\right)$, para o modelo de estatísticas de ordem Weibul comparado aos demais modelos. 
Na tabela (22), temos os critérios de seleção, definidos no Capítulo 5, para discriminar os modelos em estudos: a soma dos erros relativos (5.2.4) e os PCPOs, definidos por $\mathrm{c}(\ell)=\prod_{\mathrm{i}=1}^{\mathrm{n}} \mathrm{c}_{\mathrm{i}}$, onde $\mathrm{c}_{\mathrm{i}}=\mathrm{p}\left(\mathrm{x}_{\mathrm{i}+1} / \mathrm{D}_{\mathrm{x}_{\mathrm{i}}}\right)$ para os modelos de estatísticas de ordem Weibul, gama, gama generalizada, exponencial, log-normal e gaussiana inversa.

Tabela 22. Soma dos erros relativos e $\mathrm{c}(\ell)$ dos modelos de estatísticas de ordem gama generalizada, gama, exponencial, Weibul, log-normal e gaussiana inversa.

\begin{tabular}{|l|l|l|}
\hline \multicolumn{1}{|c|}{ Modelos } & \multicolumn{1}{c|}{$\mathrm{c}(\ell)$} & \multicolumn{1}{c|}{$\mathrm{RE}$} \\
\hline Modelo de estatísticas de ordem gama genera. & $9.56 \times 10^{-50}$ & 6.8110 \\
Modelo de estatísticas de ordem gama & $1.33 \times 10^{-51}$ & 24.112 \\
Modelo de estatísticas de ordem exponencial & $3.61 \times 10^{-57}$ & 225.414 \\
Modelo de estatísticas de ordem Weibul & $9.91 \times 10^{-58}$ & 67.45 \\
Modelo de estatísticas de ordem log-normal & $1.130 \times 10^{-51}$ & 8.1012 \\
Modelo de estatísticas de ordem gaussiana inv. & $1.011 \times 10^{-51}$ & 25.467 \\
\hline
\end{tabular}

$\mathrm{Na}$ figura (13), apresentamos os valores de $c_{i}$ versus os valores de $i$, onde $\mathrm{i}$ representa o i-ésimo intervalo de ocorrência de falhas $(\mathrm{i}=1, \ldots, 26)$, para os modelos de estatísticas de ordem gama, gama-generalizada, lognormal, Weibul, exponencial e gaussiana inversa. 


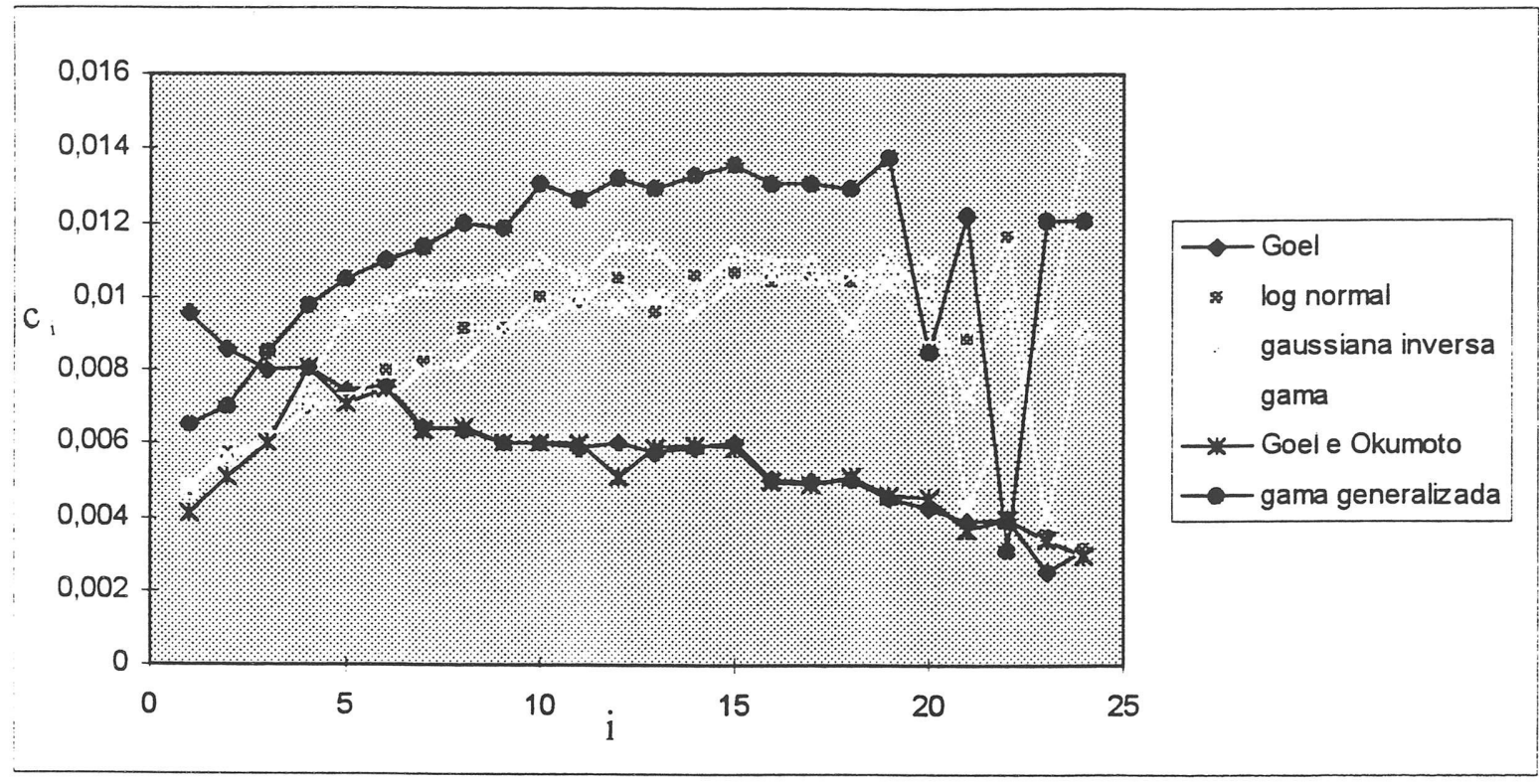

Figura 13. Valores de $c_{i}$ versus $i$ para os modelos de estatísticas de ordem gama, gama-generalizada, Weibul, log-normal, exponencial e gaussiana inversa.

\section{Adequabilidade do Modelo}

Sabemos que a construção dos modelos de estatísticas de ordem, denotados de PPNH-I, está baseada nas seguintes suposições:

- O número de erros $\mathrm{N}$ no início do teste do software tem distribuição de Poisson;

- a média do número de erros $\mathrm{N}$ é $\theta$.

Então, a função de valor médio é dada por $m\left(x_{i}\right)=\theta F\left(x_{i} \backslash \beta\right)$ (ver Capítulo 4, seção 4.1.2). Se essas suposições estiverem corretas, é fácil verificar que $\mathrm{m}\left(\mathrm{x}_{\mathrm{i}}\right) / \theta=\mathrm{F}\left(\mathrm{x}_{\mathrm{i}} \backslash \beta\right)$ tem uma distribuição Uniforme $(0,1)$.

Assim, para verificar se o modelo é adequado para os dados, ou seja, se a suposição é valida, fazemos um estudo para cada tempo de falha, construindo QXQ plots empíricos dos estimadores Monte Carlo de $\hat{\mathrm{m}}\left(\mathrm{x}_{\mathrm{i}}\right) / \hat{\theta}$ contra Uniforme $(0,1)$ para cada modelo (ver figura, 14$)$. 


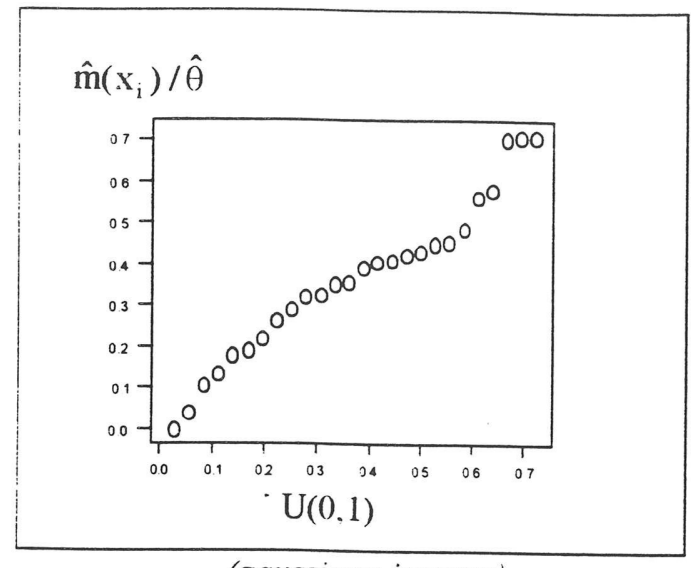

(gaussiana inversa)

$\hat{\mathrm{m}}\left(\mathrm{x}_{\mathrm{i}}\right) / \hat{\theta}$

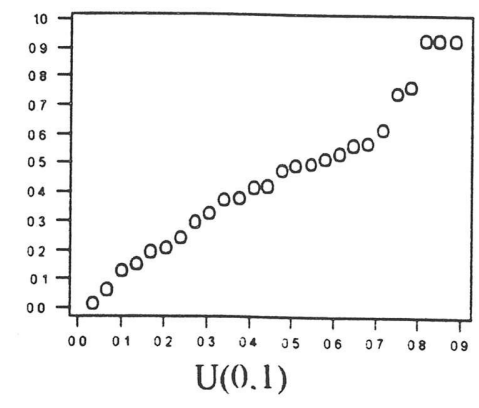

(gama)

$\hat{\mathrm{m}}\left(\mathrm{x}_{\mathrm{i}}\right) / \hat{\theta}$

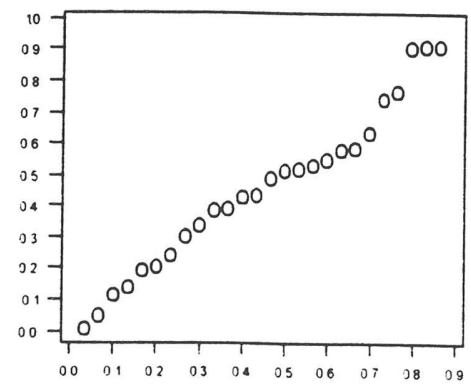

$\mathrm{U}(0.1)$

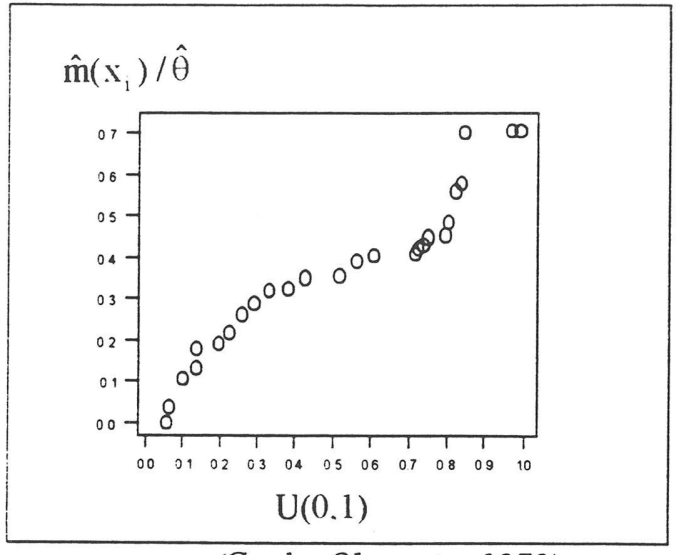

(Goel e Okumoto, 1979)

$\hat{\mathrm{m}}\left(\mathrm{x}_{\mathrm{i}}\right) / \hat{\theta}$

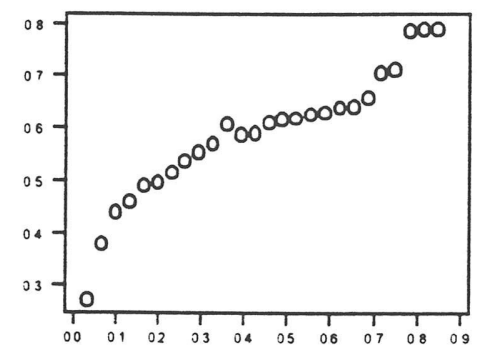

$\mathrm{U}(0.1)$

(Goel, 1983)

$\hat{\mathbf{m}}\left(\mathrm{x}_{\mathrm{i}}\right) / \hat{\theta}$

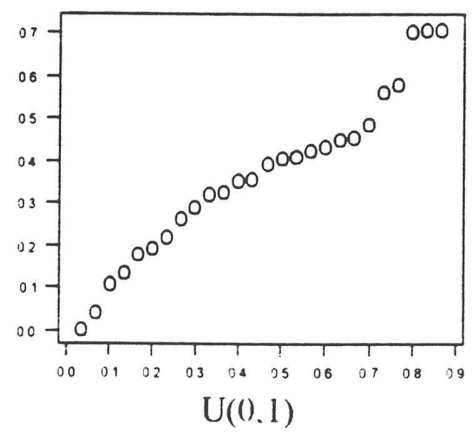

(log normal)

(gama generalizada)

Figura 14. Empíricos $Q X Q$ plots de $\hat{\mathrm{m}}\left(\mathrm{X}_{\mathrm{i}}\right) / \hat{\theta}$ versus a distribuição uniforme $(0,1)$ para os 6 modelos de estatísticas de ordem.

A título de decidir quais dos seis modelos analisados melhor explicam o fenômeno de confiabilidade crescente do software em estudo, podemos concluir, através das técnicas utilizadas, que, para este conjunto de dados, o modelo de estatísticas de ordem gama generalizada mostrou-se superior aos 
demais principalmente se comparado com os modelos especificados a partir de 2 parâmetros (modelos:Weibul e exponencial).

Fazendo a comparação entre modelos de mesma dimensão, concluímos que o modelo de estatísticas de ordem gaussiana inversa é superior ao modelo estatísticas de ordem log-normal. Quanto aos modelos especificados a partir de 2 parâmetros, podemos afirmar, através das técnicas utilizadas, que o modelo de estatísticas de ordem gama é melhor que os modelos de estatísticas de ordem Weibul e exponencial, para esse conjunto de dados (tabela (11)).

A adequação dos modelos é verificada na figura (15). Observamos uma razoável linearidade dos pontos indicando uma boa adequabilidade dos modelos analisados para esse conjunto de dados. 


\section{Capítulo 7}

\section{CONCLUSÕES E PERSPECTIVAS FUTURAS}

O uso de técnicas de simulação estocástica MCMC (cadeias de Markov com Monte Carlo) é uma forma apropriada para a análise de dados de confiabilidade de software com modelos de estratégia de tipo II. Esses modelos, assumindo um processo de Poisson homogêneo ou não homogêneo em geral, envolvem muitos parâmetros, e as inferências usuais baseadas na estimação de máxima verossimilhança não são viáveis. Por outro lado, o uso de métodos Bayesianos, usando-se algoritmos amostrador de Gibbs e Metropolis-Hastings, é de simples implementação, e não exige conhecimento computacional sofisticado. Além disso, a obtenção de quantidades a posteriori de interesse e densidades preditivas é facilmente obtida por estimadores de Monte Carlo. As técnicas Bayesianas para discriminação dos vários modelos propostos para análise dos dados de falhas, são simples de serem aplicadas e mostram-se eficientes.

As técnicas apresentadas nesta dissertação poderiam ser generalizadas a outras classes de modelos de confiabilidade de software de tipo II, considerando, ainda, a inclusão de covariáveis (informação sobre tipo de programa, programador, etc). 


\section{APÊNDICE A}

Neste apêndice, apresentamos os algoritmos amostrador de Gibbs, Metropolis-Hastings e o critério de verificação de convergência de Gelman e Rubin (1992).

\section{A.1 ALGORITMO AMOSTRADOR DE GIBBS}

O amostrador de Gibbs é uma técnica da cadeia de Markov, com estimadores Monte Carlo, para gerar variáveis aleatórias de uma distribuição sem o cálculo da densidade a posteriori (ver Gelfand e Smith, 1990). A distribuição estacionária da cadeia de Markov é a distribuição a posteriori que temos interesse em encontrar.

A seguir apresentamos o algoritmo amostrador de Gibbs.

Suponha que desejamos estimar a densidade a posteriori de $\left(\theta_{1}, \ldots, \theta_{\mathrm{p}}\right)$ condicionada aos dados, ou seja, $\mathrm{p}\left(\theta_{1}, \ldots, \theta_{\mathrm{p}} \backslash\right.$ dados $)$.

i) Inicializar o contador de iterações da cadeia $\mathrm{j}=1$ e arbitrar valores iniciais,

$$
\theta_{1}^{(0)}, \ldots, \theta_{\mathrm{p}}^{(0)}
$$

ii) Gerar um valor $\theta_{1}^{(1)}$ de uma densidade condicional

$$
\mathrm{p}\left(\theta_{1} \backslash \theta_{2}^{(0)}, \ldots, \theta_{\mathrm{p}}^{(0)}, \text { dados }\right)
$$

iii) Similarmente, gerar um valor $\theta_{2}^{(1)}$ de uma densidade condicional

$$
\mathrm{p}\left(\theta_{2} \backslash \theta_{1}^{(1)}, \theta_{2}^{(0)}, \ldots, \theta_{\mathrm{p}}^{(0)}, \text { dados }\right)
$$


iv) Continuar até gerar o valor $\theta_{\mathrm{p}}^{(1)}$ da densidade condicional

$$
\mathrm{p}\left(\theta_{\mathrm{p}} \backslash \theta_{1}^{(1)}, \theta_{2}^{(1)}, \ldots, \theta_{\mathrm{p}-1}^{(1)}, \text { dados }\right) .
$$

Considerando-se as novas realizações $\left(\theta_{1}^{(1)}, \ldots, \theta_{\mathrm{p}}^{(1)}\right)$, substitui-se os valores anteriores $\left(\theta_{1}^{(0)}, \ldots, \theta_{\mathrm{p}}^{(0)}\right)$ pelos valores novos e repete-se os passos ii), iii) e iv) iterativamente até a obtenção da convergência.

Gemam e Geman (1984) e Gelfand e Smith (1990) argumentam que, sob certas condições regulares e para um grande número de simulações, essa cadeia de Markov converge para uma distribuição estacionária. 


\section{A.2 Algoritmo de Metropolis-Hastings}

O algoritmo de Metropolis-Hastings é utilizado quando temos interesse em gerar amostras aleatórias de distribuições condicionais não padronizadas.

Supor que desejamos amostras de uma densidade não regular $\mathrm{p}\left(\theta_{\mathrm{i}} \backslash \theta_{1}, \theta_{2}, \ldots, \theta_{\mathrm{i}-1}, \theta_{\mathrm{i}+1}, \ldots, \theta_{\mathrm{k}}\right)$ ou simplesmente $\mathrm{p}\left(\theta_{\mathrm{i}} \backslash \theta^{(\mathrm{j})}\right)$ onde $\theta^{(\mathrm{j})}$ è $\theta_{1}, \theta_{2}, \ldots, \theta_{\mathrm{i}-1}, \theta_{\mathrm{i}+1}, \ldots, \theta_{\mathrm{k}}$.

Definir o núcleo de transição $q(\theta, \beta)$, da distribuição $p(\theta)$, que gera $\beta$ a partir de $\theta$.

Quando a posteriori é escrita na forma $\pi(\mathrm{t})=\Psi(\mathrm{t}) \mathrm{h}(\mathrm{t})$, onde $\mathrm{h}(\mathrm{t})$ é uma densidade que pode ser amostrada e $\Psi(\mathrm{t})$ é uniformemente limitada. Então $h(t)=q(\theta, \beta)$ é usado como candidato gerador.

Apresentamos a seguir o algoritmo Metropolis-Hastings.

1) Iniciar com um valor $\theta^{(0)}$ e indicador de estágio $j=0$;

2) Gerar um ponto $\beta$ de acordo com o núcleo de transição $q\left(\theta^{(j)}, \beta\right)$;

3) Atualizar $\theta^{(j)}$ por $\theta^{(j+1)}=\beta$ com probabilidade

$$
p=\min \left\{1, \frac{p(\beta), q\left(\theta^{(j)}, \beta\right)}{p\left(\theta^{(j)}\right) q\left(\beta, \theta^{(j)}\right)}\right\}
$$

4) Repetir estágios 2) e 3) até obter uma distribuição estacionária. 


\section{A.3 Critério de Gelman e Rubin, 1992}

Como discutido anteriormente, a convergência (em distribuição) dos algoritmos amostrador de Gibbs e Metropolis-Hastings, pode ser verificada de várias formas. O critério apresentado a seguir foi proposto por Gelman e Rubin (1992).

O critério de Gelman e Rubin (1992), considera que, cada uma das m seqüências têm comprimento $\mathrm{M}+\mathrm{N}$. Descartamos as $\mathrm{M}$ primeiras amostras e focalizamos atenção nas $\mathrm{N}$ últimas amostras. Daí calculamos,

$$
\frac{U}{N}=\frac{\sum_{i=1}^{N}\left(\bar{\beta}_{i}-\bar{\beta}_{. . .}\right)^{2}}{(m-1)}
$$

onde $\bar{\beta}_{\mathrm{i}}$. são m médias baseadas nas últimas iterações da seqüência e

$$
\mathrm{W}=\frac{\sum_{\mathrm{i}=1}^{\mathrm{N}} \mathrm{s}_{\mathrm{i}}^{2}}{\mathrm{~m}},
$$

onde $s_{i}^{2}=\frac{\sum_{i=1}^{N}\left(\beta_{i}-\bar{\beta}_{i}\right)^{2}}{(N-1)}$.

Observar que $\mathrm{U} / \mathrm{N}$ representa a variabilidade entre as $\mathrm{m}$ seqüências e W representa a média das $\mathrm{N}$ variâncias dentro das seqüências.

Daí, a variância da distribuição estudada pode ser estimada por:

$$
\hat{\sigma}^{2}=\frac{N-1}{N} W+\frac{1}{N} U
$$


e a média estimada por $\hat{\mu}=\bar{\beta}_{\text {... }}$.

Portanto temos o resultado que a distribuição de $\beta$ condicionada aos dados é aproximadamente $t$ - Student com centro $\hat{\mu}=\bar{\beta}$.. e desvio padrão dado por:

$$
\sqrt{\hat{V}}=\sqrt{\sigma^{2}+\mathrm{U} / \mathrm{Nm}}
$$

e graus de liberdade $d f=\frac{2 \hat{V}^{2}}{\operatorname{VAR}(\hat{V})}$,

onde,

$$
\begin{aligned}
& \operatorname{VÂR}(\hat{V})=\left(\frac{N-1}{N}\right)^{2} \frac{1}{N} \operatorname{VÂR}\left(s_{i}^{2}\right)+\left(\frac{m+1}{m+N}\right)^{2} \frac{2}{m-1} U^{2}+ \\
& 2 \frac{(m+1)(N-1)}{m N} \frac{N}{m}\left[\operatorname{cov}\left(s_{i}^{2}, \bar{\beta}_{i}^{2}\right)-2 \bar{\beta}_{. .} \operatorname{cov}\left(s_{i}^{2}, \bar{\beta}_{i} .\right)\right]
\end{aligned}
$$

Para verificarmos a convergência em distribuição utilizamos o fator de redução de escala dado por $\sqrt{\hat{R}}=\sqrt{\frac{d f}{(d f-2)} \frac{\hat{V}}{W}}$, se esse fator é maior que 1 , devemos considerar mais simulações para melhorar a inferência sobre a distribuição de interesse, se $\sqrt{\hat{\mathrm{R}}}$ estiver próximo de 1, Gelman e Rubin (1992), argumentam que a convergência foi atingida e as amostras selecionadas das iterações são i.i.d. 


\section{APÊNDICE B}

Neste apêndice, apresentamos um dos programas computacionais utilizados nesta dissertação. O programa foi desenvolvido utilizando o pacote $M A T L A B$. Foram utilizados alguns módulos principais do pacote, como por exemplo o STATS, OPS, GRAPHICS, etc. O programa apresentado é o algoritmo amostrador de Gibbs e Metropolis-Hastings para o modelo de estatísticas de ordem gaussiana inversa, que foi utilizado para encontrar as densidades a posteriori marginais para $\mathrm{N}^{\prime}, \theta, \lambda$ e $\mu$, a soma dos erros relativos desse modelo e o critério de parada de Gelman e Rubin (1992) de $\mathrm{N}^{\prime}, \theta, \lambda$ e $\mu$.

PROGRAMA DO ALGORITMO DE METROPOLIS-HASTINGS E AMOSTRADOR DE GIBBS PARA O MODELO DE ESTATÍSTICAS DE ORDEM GAUSSINA INVERSA

format long e

clear

cont $1=0$;

cont $2=0$;

rand('seed', 0);

$\mathrm{t}=\left[\begin{array}{llll}9 & 213236434550586370717778 & 3791929598104105116149156247\end{array}\right.$

$249250]$;

al $=756$;

$\mathrm{b} 1=1 / 2.75$;

a2 $=100$;

b2=1;

a3 $=107$;

$\mathrm{b} 3=1 / 3.25$;

$\mathrm{n}(1)=10$;

$\operatorname{teta}(1)=26$;

lambda $(1)=100$;

$\operatorname{mi}(1)=275$; 
$\%$

\% Condições iniciais

$\%$

$\mathrm{v}=\left[\begin{array}{llllllllll}10 & 12 & 16 & 18 & 14 & 11 & 13 & 9 & 19 & 8\end{array}\right.$

26212223242925323019

10014510510910611810712098110

275248258199250240210289247 220];

for $\mathrm{k}=2: 12001$

if $\operatorname{rem}(\mathrm{k}-2,1200)==0 \& \mathrm{k}<1200$

$\mathrm{n}(\mathrm{k}-1)=\mathrm{v}(1,1+(\mathrm{k}-2) / 1200)$;

teta $(\mathrm{k}-1)=\mathrm{v}(2,1+(\mathrm{k}-2) / 1200)$;

lambda $(\mathrm{k}-1)=\mathrm{v}(3,1+(\mathrm{k}-2) / 1200)$;

$\mathrm{mi}(\mathrm{k}-1)=\mathrm{v}(4,1+(\mathrm{k}-2) / 1200)$;

end

\% Cálculo do Somatório

for $j=1: 26$

$\left.\operatorname{st} 1 \mathrm{~m}=\operatorname{sum}\left(((\mathrm{t}-\mathrm{mi}(\mathrm{k}-1)))^{\wedge} 2\right) . / \mathrm{t}\right)$;

end

\% Cálculo da Função distribuição

$\mathrm{xl}=\operatorname{sqrt}(\mathrm{lambda}(\mathrm{k}-1) / 250) *(250 / \mathrm{mi}(\mathrm{k}-1)-1)$;

x2=-sqrt(lambda $(\mathrm{k}-1) / 250) *(1+250 / \mathrm{mi}(\mathrm{k}-1))$;

$\mathrm{pl}=\operatorname{normcdf}(\mathrm{xl})$

$\mathrm{p} 2=\operatorname{normcdf}(\mathrm{x} 2)$

$\mathrm{g}=\mathrm{pl}+\mathrm{p} 2 * \exp (2 * \operatorname{lambda}(\mathrm{k}-1) / \mathrm{mi}(\mathrm{k}-1))$;

\% Parâmetro da teta

$A=a 3+n(k-1)$

$\%$

$\%$ Gibbs Sampling (teta)

$\%$

\% Geração da Gama

$\operatorname{teta}(\mathrm{k})=\operatorname{gamrnd}(\mathrm{A}, \mathrm{b} 3)$; 
\%-----.------------

$\% \quad$ Gibbs Sampling (n)

\%-------------------------

\% Geração da Poisson

theta=teta $(\mathrm{k}) *(1-\mathrm{g})$;

$n(k)=$ poissrnd (theta);

\%--------------------------

$\%$ Metrópolis Hastings (mi)

\%-------------------------------

\% Geração da Gama

$\mathrm{y}=\operatorname{gamrnd}(\mathrm{al}, \mathrm{bl})$

\% Cálculo do somatório com o valor atualizado (y)

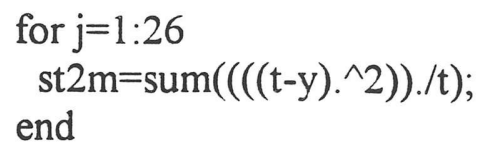

\% Cálculo da Função distribuição com o valor mi atualizado

x11=sqrt(lambda(k-1)/250)*(250/y-1);

$\mathrm{x} 22=-\operatorname{sqrt}(\operatorname{lambda}(\mathrm{k}-1) / 250) *(1+250 / \mathrm{y})$;

p11 =normcdf $(x 11)$;

p22=normcdf $(x 22)$;

$\mathrm{gl}=(\mathrm{pl} 1+\mathrm{p} 22 * \exp ((2 * \operatorname{lambda}(\mathrm{k}-1)) / \mathrm{y}))$;

$\%$ Testando o valor gerado para mi $(y)$

psi $1=\exp \left(n(k) * \log (1-\mathrm{g} 1)-\operatorname{lambda}(\mathrm{k}-1) * \operatorname{st} 2 \mathrm{~m} /\left(2 *\left(\mathrm{y}^{\wedge} 2\right)\right)-\mathrm{n}(\mathrm{k}) * \log (1-\mathrm{g})+\operatorname{lambda}(\mathrm{k}-\right.$

$\left.1)^{*} \operatorname{st~} \operatorname{lm} /\left(2 * \operatorname{mi}(\mathrm{k}-1)^{\wedge} 2\right)\right)$;

$\mathrm{ml}=\min (1, \mathrm{psil})$;

rand('uniform');

um=rand:

if um $<=\mathrm{m} 1$

$\operatorname{mi}(\mathrm{k})=\mathrm{y}$;

cont $1=$ cont $1+1$;

else

$\operatorname{mi}(k)=\operatorname{mi}(k-1)$;

end 
for $j=1: 26$

$\operatorname{st} 22 \mathrm{~m}=\operatorname{sum}\left(\left((\mathrm{t}-\mathrm{mi}(\mathrm{k})) \cdot{ }^{\wedge} 2\right) \cdot / \mathrm{t}\right)$;

end

\% Cálculo da Função distribuição utilizando mi(k)

x111=sqrt(lambda(k-1)/250)*(250/mi(k)-1);

x222=-sqrt(lambda(k-1)/250)*(1+250/mi(k));

p111=normcdf $(x 111)$;

p222=normcdf $(x 222)$;

$\mathrm{ga}=(\mathrm{p} 111+\mathrm{p} 222 * \exp ((2 * \operatorname{lambda}(\mathrm{k}-1)) / \mathrm{mi}(\mathrm{k})))$;

$\%$

$\%$ Metrópolis Hastings (lambda)

$\%$

$\mathrm{z}=\operatorname{gamrnd}(\mathrm{a} 2,1 / \mathrm{b} 2)$

\% Cálculo da função distribuição utilizando (z)

$\mathrm{f}=\operatorname{sqrt}(\mathrm{z} / 250) *(250 / \mathrm{mi}(\mathrm{k})-1)$;

$\mathrm{fl}=-\operatorname{sqrt}(\mathrm{z} / 250)^{*}(1+250 / \mathrm{mi}(\mathrm{k}))$;

$\mathrm{f} 7=\operatorname{normcdf}(\mathrm{f})$;

$\mathrm{f} 9=\operatorname{normcdf}(\mathrm{fl})$;

$\mathrm{g} 21=(\mathrm{f} 7+\mathrm{f} 9 * \exp ((2 * \mathrm{z}) / \mathrm{mi}(\mathrm{k})))$;

$\%$ Testando o valor para lambda $(z)$

$\mathrm{psi} 2=\exp \left(13 * \log (\mathrm{z})+\mathrm{n}(\mathrm{k}) * \log (1-\mathrm{g} 21)-\mathrm{z}^{*} \mathrm{st} 22 \mathrm{~m} /(2 *(\mathrm{mi}(\mathrm{k}) \wedge 2))-13 * \log (\operatorname{lambda}(\mathrm{k}-1))-\right.$ $\mathrm{n}(\mathrm{k}) * \log (1-\mathrm{ga})+\operatorname{lambda}(\mathrm{k}-1) * \mathrm{st} 22 \mathrm{~m} /(2 *(\mathrm{mi}(\mathrm{k}) \wedge 2)))$;

$\mathrm{m} 2=\min (1, \mathrm{psi} 2)$;

rand('uniform');

uml $=$ rand;

if uml $<=\mathrm{m} 2$

lambda $(\mathrm{k})=\mathrm{z}$;

cont $2=$ cont $2+1$;

else

lambda $(\mathrm{k})=\mathrm{lambda}(\mathrm{k}-1)$;

end

\% Cálculo da função distribuição utilizando lambda(k)

$\mathrm{j}=\operatorname{sqrt}(\mathrm{lambda}(\mathrm{k}) / 250) *(250 / \mathrm{mi}(\mathrm{k})-1)$;

$\mathrm{j} 1=-\operatorname{sqrt}(\mathrm{lambda}(\mathrm{k}) / 250) *(1+250 / \mathrm{mi}(\mathrm{k}))$;

$\mathrm{j} 7=\operatorname{normcdf}(\mathrm{j})$; 
$j 9=\operatorname{normcdf}(j 1)$;

$\mathrm{g} 311=(\mathrm{j} 7+\mathrm{j} 9 * \exp (\mathrm{lambda}(\mathrm{k}) / \mathrm{mi}(\mathrm{k})))$;

end

$\%$

\% Seleção da Amostra

$\%$

$\mathrm{c}=1$;

for $\mathrm{j}=201: 1200: 12000$;

for $\mathrm{i}=\mathrm{j}: \mathrm{j}+999$;

if $\operatorname{rem}(\mathrm{i}-\mathrm{j}+1,10)=0$

lambdas(c)=lambda(i);

$\operatorname{mis}(\mathrm{c})=\operatorname{mi}(\mathrm{i})$;

$\operatorname{tetas}(\mathrm{c})=\operatorname{teta}(\mathrm{i})$;

$\mathrm{ns}(\mathrm{c})=\mathrm{n}(\mathrm{i})$;

$\mathrm{c}=\mathrm{c}+1$;

end

end

end

\% Critério de Parada para Lambda

$\mathrm{nc}=10$

$\mathrm{nl}=1$;

$\mathrm{n} 2=100$;

$\mathrm{d}=100$;

Melambda $=[\operatorname{mean}(\operatorname{lambdas}(\mathrm{n} 1: \mathrm{n} 2))$, mean(lambdas $(\mathrm{n} 1+\mathrm{d}: \mathrm{n} 2+\mathrm{d}))$, mean $(\operatorname{lambdas}(\mathrm{n} 1+2 * \mathrm{~d}: \mathrm{n} 2+$ $2 * d)$ ), mean(lambdas $(n 1+3 * d: n 2+3 * d))$, mean (lambdas $(n 1+4 * d: n 2+4 * d)$ ), mean(lambdas $(n 1+$ $5 * \mathrm{~d}: \mathrm{n} 2+5 * \mathrm{~d})$ ), mean (lambdas $(\mathrm{n} 1+6 * \mathrm{~d}: \mathrm{n} 2+6 * \mathrm{~d})$ ), mean(lambdas $(\mathrm{n} 1+7 * \mathrm{~d}: \mathrm{n} 2+7 * \mathrm{~d})$ ), mean(lamb das(n1+8*d:n2+8*d)), mean(lambdas(n1+9*d:n2+9*d))];

QMlambda=Melambda. $*$ Melambda;

Valambda $=[\operatorname{cov}($ lambdas $(\mathrm{n} 1: \mathrm{n} 2)), \operatorname{cov}($ lambdas $(\mathrm{n} 1+\mathrm{d}: \mathrm{n} 2+\mathrm{d}))$, $\operatorname{cov}($ lambdas $(n 1+2 * d: n 2+2 * d)), \operatorname{cov}($ lambdas $(n 1+3 * d: n 2+3 * d))$,

$\operatorname{cov}(\operatorname{lambdas}(\mathrm{n} 1+4 * \mathrm{~d}: \mathrm{n} 2+4 * \mathrm{~d}))$, $\operatorname{cov}(\operatorname{lambdas}(\mathrm{n} 1+5 * \mathrm{~d}: \mathrm{n} 2+5 * \mathrm{~d})), \operatorname{cov}(\operatorname{lambdas}(\mathrm{n} 1+6 * \mathrm{~d}: \mathrm{n} 2+6 *$

d)), $\operatorname{cov}(\operatorname{lambdas}(\mathrm{n} 1+7 * \mathrm{~d}: \mathrm{n} 2+7 * \mathrm{~d})), \operatorname{cov}($ lambdas $(\mathrm{n} 1+8 * \mathrm{~d}: \mathrm{n} 2+8 * \mathrm{~d})), \operatorname{cov}($ lambdas $(\mathrm{n} 1+9 * \mathrm{~d}: \mathrm{n} 2$ $+9 * d))$;

MeMlambda=sum(Melambda)/nc;

Wlambda=sum(Valambda $) /$ nc;

VarVlambda $=($ Valambda*Valambda'-nc*Wlambda^2)/(nc-1);

Ulambda $=d^{*}($ Melambda*Melambda'-nc*MeMlambda`2)/(nc-1);

VARlambda $=((\mathrm{d}-1) / \mathrm{d}) *$ Wlambda $+(1 / \mathrm{d}) *$ Ulambda;

VHlambda=VARlambda+Ulambda/(nc*d);

Mcovlambda $=\operatorname{cov}($ Valambda,Melambda); 
Qcovlambda $=\operatorname{cov}($ Valambda, QMlambda);

COVlambda $=$ Qcovlambda $(1,2)-2 *$ MeMlambda*Mcovlambda $(1,2)$;

$\operatorname{VarVHlambda}=((\mathrm{d}-1) / \mathrm{d}) \wedge 2 *(1 / \mathrm{nc}) * \operatorname{Var} V l a m b d a+\left(((\mathrm{nc}+1) /(\mathrm{nc} * \mathrm{~d}))^{\wedge} 2\right) *(2 /(\mathrm{nc}-$

1))*Ulambda. ${ }^{\wedge} 2+2 *$ COVlambda $^{*}(\mathrm{nc}+1) *(\mathrm{~d}-1) /\left(\mathrm{d}^{*} \mathrm{nc}^{\wedge} 2\right)$;

dflambda $=2 *($ VHlambda^2)/VarVHlambda;

Rlambda $=$ sqrt((VHlambda/Wlambda)*dflambda/(dflambda-2));

\% Critério de Parada para Teta

Meteta $=[\operatorname{mean}(\operatorname{tetas}(\mathrm{n} 1: \mathrm{n} 2))$, mean $(\operatorname{tetas}(\mathrm{n} 1+\mathrm{d}: \mathrm{n} 2+\mathrm{d}))$, mean(tetas $(\mathrm{n} 1+2 * \mathrm{~d}: \mathrm{n} 2+2 * \mathrm{~d}))$, mean (te $\operatorname{tas}(n 1+3 * d: n 2+3 * d))$, mean (tetas $(n 1+4 * d: n 2+4 * d))$, mean(tetas(n1+5*d:n2+5*d)), mean(teta $\mathrm{s}(\mathrm{n} 1+6 * \mathrm{~d}: \mathrm{n} 2+6 * \mathrm{~d}))$, mean $(\operatorname{tetas}(\mathrm{n} 1+7 * \mathrm{~d}: \mathrm{n} 2+7 * \mathrm{~d}))$, mean $(\operatorname{tetas}(\mathrm{n} 1+8 * \mathrm{~d}: \mathrm{n} 2+8 * \mathrm{~d}))$, mean $(\operatorname{tetas}($ $\mathrm{nl}+9 * \mathrm{~d}: \mathrm{n} 2+9 * \mathrm{~d}))]$;

QMteta $=$ Meteta.*Meteta;

Vateta $=[\operatorname{cov}(\operatorname{tetas}(n 1: n 2)), \operatorname{cov}(\operatorname{tetas}(n 1+d: n 2+d)), \operatorname{cov}(\operatorname{tetas}(n 1+2 * d: n 2+2 * d))$, $\operatorname{cov}(\operatorname{tetas}(\mathrm{n} 1+3 * \mathrm{~d}: \mathrm{n} 2+3 * \mathrm{~d}))$,

$\operatorname{cov}(\operatorname{tetas}(\mathrm{n} 1+4 * \mathrm{~d}: \mathrm{n} 2+4 * \mathrm{~d})), \operatorname{cov}(\operatorname{tetas}(\mathrm{n} 1+5 * \mathrm{~d}: \mathrm{n} 2+5 * \mathrm{~d})), \operatorname{cov}(\operatorname{tetas}(\mathrm{n} 1+6 * \mathrm{~d}: \mathrm{n} 2+6 * \mathrm{~d})), \operatorname{cov}($ tet as $(n 1+7 * d: n 2+7 * d)), \operatorname{cov}(\operatorname{tetas}(n 1+8 * d: n 2+8 * d)), \operatorname{cov}(\operatorname{tetas}(n 1+9 * d: n 2+9 * d))]$;

MeMteta=sum(Meteta)/nc;

Wteta=sum(Vateta)/nc;

VarVteta $=($ Vateta*Vateta'-nc*Wteta^2)/(nc-1);

Uteta $=d^{*}($ Meteta*Meteta'-nc*MeMteta^2)/(nc-1);

VARteta $=((\mathrm{d}-1) / \mathrm{d}) *$ Wteta $+(1 / \mathrm{d}) *$ Uteta;

VHteta=VARteta+Uteta/(nc*d);

Mcovteta $=\operatorname{cov}($ Vateta, Meteta);

Qcovteta $=\operatorname{cov}($ Vateta, QMteta);

COVteta $=$ Qcovteta $(1,2)-2 *$ MeMteta*Mcovteta $(1,2)$;

$\left.\operatorname{VarVHteta}=((\mathrm{d}-1) / \mathrm{d}) \wedge 2 *(1 / \mathrm{nc})^{*} \operatorname{VarVteta}+((\mathrm{nc}+1) /(\mathrm{nc} * \mathrm{~d}))^{\wedge} 2\right)^{*}(2 /(\mathrm{nc}-$

1))*Uteta^2+2*COVteta* $(n c+1) *(d-1) /\left(d^{*} n c^{\wedge} 2\right)$;

dfteta $=2 *($ VHteta 2$) /$ VarVHteta;

Rteta $=$ sqrt $(($ VHteta/Wteta $) *$ dfteta/(dfteta-2))

$\%$ Critério de Parada para mi

Memi $=[\operatorname{mean}(\operatorname{mis}(\mathrm{n} 1: \mathrm{n} 2)), \operatorname{mean}(\operatorname{mis}(\mathrm{n} 1+\mathrm{d}: \mathrm{n} 2+\mathrm{d})), \operatorname{mean}(\operatorname{mis}(\mathrm{n} 1+2 * \mathrm{~d}: \mathrm{n} 2+2 * \mathrm{~d})), \operatorname{mean}(\operatorname{mis}(\mathrm{nl}$ $+3 * \mathrm{~d}: \mathrm{n} 2+3 * \mathrm{~d}))$, mean $(\operatorname{mis}(\mathrm{nl}+4 * \mathrm{~d}: \mathrm{n} 2+4 * \mathrm{~d}))$, mean $(\operatorname{mis}(\mathrm{n} 1+5 * \mathrm{~d}: \mathrm{n} 2+5 * \mathrm{~d}))$, mean $(\operatorname{mis}(\mathrm{nl}+6 * \mathrm{~d}$ : $\mathrm{n} 2+6 * \mathrm{~d}))$, mean $(\operatorname{mis}(\mathrm{n} 1+7 * \mathrm{~d}: \mathrm{n} 2+7 * \mathrm{~d}))$, mean $(\operatorname{mis}(\mathrm{n} 1+8 * \mathrm{~d}: \mathrm{n} 2+8 * \mathrm{~d}))$, mean $(\operatorname{mis}(\mathrm{n} 1+9 * \mathrm{~d}: \mathrm{n} 2+9$

$* d))$;

$\mathrm{QMmi}=$ Memi. ${ }^{*}$ Memi;

$\operatorname{Vami}=[\operatorname{cov}(\operatorname{mis}(n 1: n 2)), \operatorname{cov}(\operatorname{mis}(n 1+d: n 2+d)), \operatorname{cov}(\operatorname{mis}(n 1+2 * d: n 2+2 * d))$, $\operatorname{cov}(\operatorname{mis}(\mathrm{n} 1+3 * \mathrm{~d}: \mathrm{n} 2+3 * \mathrm{~d}))$,

$\operatorname{cov}(\operatorname{mis}(\mathrm{n} 1+4 * d \cdot n 2+4 * d)), \operatorname{cov}(\operatorname{mis}(\mathrm{n} 1+5 * d \cdot \mathrm{n} 2+5 * d)), \operatorname{cov}(\operatorname{mis}(\mathrm{n} 1+6 * d \cdot n 2+6 * d)), \operatorname{cov}(\operatorname{mis}(\mathrm{nl}$ $+7 * d: n 2+7 * d)), \operatorname{cov}(\operatorname{mis}(n 1+8 * d: n 2+8 * d)), \operatorname{cov}(\operatorname{mis}(n 1+9 * d: n 2+9 * d))]$;

$\mathrm{MeMmi}=\operatorname{sum}(\mathrm{Memi}) / \mathrm{nc}$;

$\mathrm{Wmi}=\operatorname{sum}(\mathrm{Vami}) / \mathrm{nc}$;

VarVmi $=($ Vami*Vami'-nc*Wmi^2)/(nc-1);

$\mathrm{Umi}=\mathrm{d}^{*}\left(\mathrm{Memi}^{*}{ }^{*} \mathrm{Memi}^{\prime}-\mathrm{nc}{ }^{*} \mathrm{MeMmi}^{\wedge} 2\right) /(\mathrm{nc}-1)$; 
VARmi $=((\mathrm{d}-1) / \mathrm{d}) * \mathrm{Wmi}+(1 / \mathrm{d}) * \mathrm{Umi}$;

$\mathrm{VHmi}=\mathrm{VARmi}+\mathrm{Umi} /\left(\mathrm{nc}^{*} \mathrm{~d}\right)$;

Mcovmi $=\operatorname{cov}($ Vami,Memi);

Qcovmi $=\operatorname{cov}($ Vami,QMmi);

COVmi=Qcovmi $(1,2)-2 * \mathrm{MeMmi} * \operatorname{Mcovmi}(1,2)$;

$\operatorname{VarVHmi}=((\mathrm{d}-1) / \mathrm{d})^{\wedge} 2 *(1 / \mathrm{nc}) * \operatorname{Var} \operatorname{Vmi}+\left(((\mathrm{nc}+1) /(\mathrm{nc} * \mathrm{~d}))^{\wedge} 2\right) *(2 /(\mathrm{nc}-$

1))*Umi^2+2* $\operatorname{COVmi}^{*}(\mathrm{nc}+1) *(\mathrm{~d}-1) /\left(\mathrm{d}^{*} \mathrm{nc}^{\wedge} 2\right)$;

$\mathrm{dfmi}=2 *\left(\mathrm{VHmi}^{\wedge} 2\right) / \mathrm{VarVHmi}$;

$\mathrm{Rmi}=\operatorname{sqrt}((\mathrm{VHmi} / \mathrm{Wmi}) * \mathrm{dfmi} /(\mathrm{dfmi}-2))$

\%Critério de Parada para $n$

Men $=[\operatorname{mean}(\mathrm{ns}(\mathrm{n} 1: \mathrm{n} 2))$, mean $(\mathrm{ns}(\mathrm{n} 1+\mathrm{d}: \mathrm{n} 2+\mathrm{d}))$, mean $(\mathrm{ns}(\mathrm{n} 1+2 * \mathrm{~d}: \mathrm{n} 2+2 * \mathrm{~d}))$, mean $(\mathrm{ns}(\mathrm{n} 1+3 * \mathrm{~d}$ : $n 2+3 * d))$, mean(ns(n1+4*d:n2+4*d)), mean(ns(n1+5*d:n2+5*d)), mean(ns(n1+6*d:n2+6*d)), mean(ns(n1+7*d:n2+7*d)), mean(ns(n1+8*d:n2+8*d)), mean(ns(n1+9*d:n2+9*d))];

$\mathrm{QMn}=\mathrm{Men} .{ }^{*} \mathrm{Men}$;

$\operatorname{Van}=[\operatorname{cov}(n s(n 1: n 2)), \operatorname{cov}(n s(n 1+d: n 2+d)), \operatorname{cov}(n s(n 1+2 * d: n 2+2 * d))$, $\operatorname{cov}(\mathrm{ns}(\mathrm{n} 1+3 * \mathrm{~d}: \mathrm{n} 2+3 * \mathrm{~d}))$,

$\operatorname{cov}(n s(n 1+4 * d: n 2+4 * d)), \operatorname{cov}(n s(n 1+5 * d: n 2+5 * d)), \operatorname{cov}(n s(n 1+6 * d: n 2+6 * d)), \operatorname{cov}(n s(n 1+7 *$ $\mathrm{d}: \mathrm{n} 2+7 * d)), \operatorname{cov}(\mathrm{ns}(\mathrm{n} 1+8 * d \cdot n 2+8 * d)), \operatorname{cov}(\mathrm{ns}(\mathrm{n} 1+9 * \mathrm{~d}: \mathrm{n} 2+9 * d))]$;

$\mathrm{MeMn}=\operatorname{sum}(\mathrm{Men}) / \mathrm{nc}$;

$\mathrm{Wn}=\operatorname{sum}(\operatorname{Van}) / \mathrm{nc}$;

$\operatorname{VarVn}=\left(\operatorname{Van} * \operatorname{Van}-\mathrm{nc}^{*} \mathrm{Wn}^{\wedge} 2\right) /(\mathrm{nc}-1)$;

$\mathrm{Un}=\mathrm{d}^{*}\left(\mathrm{Men} * \mathrm{Men}^{\prime}-\mathrm{nc} * \mathrm{MeMn}^{\wedge} 2\right) /(\mathrm{nc}-1)$;

$\operatorname{VAR} n=((\mathrm{d}-1) / \mathrm{d}) * \mathrm{Wn}+(1 / \mathrm{d}) * \mathrm{Un}$;

$\mathrm{VHn}=\mathrm{VARn}+\mathrm{Un} /(\mathrm{nc} * \mathrm{~d})$;

Mcovn=cov(Van,Men);

Qcovn= $\operatorname{cov}(\operatorname{Van}, \mathrm{QMn})$;

$\operatorname{COVn}=\mathrm{Q} \operatorname{covn}(1,2)-2 * \mathrm{MeMn} * \operatorname{Mcovn}(1,2)$;

$\operatorname{VarVHn}=((\mathrm{d}-1) / \mathrm{d}) \wedge 2 *(1 / \mathrm{nc})^{*} \operatorname{Var} \operatorname{Vn}+\left(((\mathrm{nc}+1) /(\mathrm{nc} * \mathrm{~d}))^{\wedge} 2\right) *(2 /(\mathrm{nc}-$

1) $) * \mathrm{Un}^{\wedge} 2+2 * \operatorname{COVn} *(\mathrm{nc}+1) *(\mathrm{~d}-1) /\left(\mathrm{d}^{*} \mathrm{nc}^{\wedge} 2\right)$;

$\mathrm{dfn}=2 *\left(\mathrm{VHn}^{\wedge} 2\right) / \mathrm{VarVHn}$;

$\mathrm{Rn}=\operatorname{sqrt}((\mathrm{VHn} / \mathrm{Wn}) * \mathrm{dfn} /(\mathrm{dfn}-2))$

$\%$

$\%$ Soma dos erros Relativos

$\%$

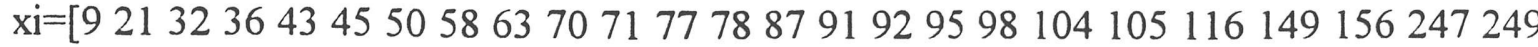
250];

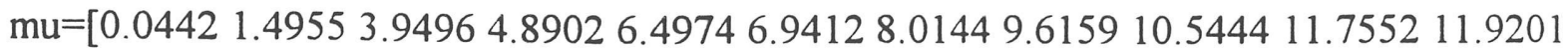
12.869613 .021414 .311814 .844214 .973715 .353515 .721216 .422016 .534617 .6994 $20.541221 .044725 .676825 .750725 .7873]$;

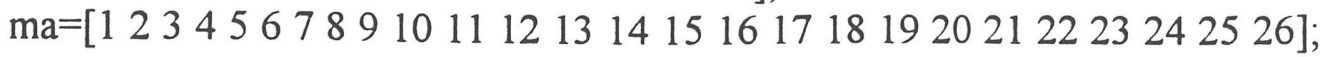

hq=lambda. ${ }^{\wedge}(1 / 2)$; 
hqa $=(9 . \wedge(-1 / 2))$;

hql $=$ hq. ${ }^{*}$ (hqa);

hq2 $=($ mi..$(-1)) * 9$;

hq3 $=($ hq2-1);

hq4=hq1. ${ }^{*}$ hq3;

$\mathrm{pp}=$ normcdf(hq4);

hq $6=(1+$ hq 2$)$;

hq7=-hq1. ${ }^{*}$ hq6;

pp1=normcdf(hq7);

hq8=lambda. ${ }^{*} 2$;

hq9 $=$ hq $8 . *$ mi..$^{\wedge}(-1)$;

pp3=exp(hq9);

$\mathrm{g} 70=(\mathrm{pp}+\mathrm{pp} 1 . * \mathrm{pp} 3)$;

$\mathrm{mc}=(\mathrm{g} 70 . *$ teta $)$;

$\mathrm{mcl}=\operatorname{sum}(\mathrm{mc})$;

$\mathrm{R} 1=\left((\mathrm{ma}-\mathrm{mc}) \cdot{ }^{\wedge} 2\right) \cdot{ }^{*}(\mathrm{mc}) . \wedge(-1)$;

$\mathrm{R} 4=\operatorname{sum}(\mathrm{R} 1)$;

end

$\%$ -

$\%$ Adequabilidade do Modelo

$\%$

$\mathrm{AM}=\mathrm{ma} / \mathrm{hq} 10$; plot(g70,AM); plot(mc/hq10,AM); $\mathrm{AM}=1 *\left(\mathrm{xi} .^{\wedge}(-1)\right)$; $\operatorname{plot}(\mathrm{g} 70, \mathrm{AM})$;

plot(mc/hq10,AM); 


\section{Bibliografia}

Dover.

ABRAMOWITZ, M., STEGUN, I. (1972). Handbook of mathematical functions,

ACHCAR, J.A. (1995). "A generalized Moranda software reliability model: a Bayesian approach", Notas do ICMSC-USP, N²2.

ACHCAR, J.A., DIPAK K. e NIVERTHY, M. (1996). "A Bayesian Approach Using Nonhomogeneous Poisson Process for Software Reliability Models", Technical Report 96-07.

ACHCAR, J.A., STORANI, K. (1996). 'Nonhomogeneous Poisson Processes Assuming a Inverse Gaussian Order Statistics Model for Software Reliability Data: a Bayesian Approach", Notas do ICMSC-USP, N 35.

BOX, G.E.P. (1980). 'Sampling and Bayes Inference in Scientific Modeling and Robusteness", Journal of the Royal Statistical Society, A, 143, 382-430.

BOX, G.E.P.; TIAO, G.C (1973). Bayesian Inference in Statistical Analysis. Addison-Wesley: Reading, Mass.

CAMPODONICO, S. e SINGPURWALLA, N.D. (1994). "A Bayesian and failure analysis of the logarithm- Poisson execution time model basead on expert opinion and failure data", IEEE Transactions on software engineering, 20, 9, 677-683.

CAMPODONICO, S. e SINGPURWALLA, N.D. (1995). "Inference and predictions from Poisson point processes incorporating expert knowledge", Journal of the Americam Statistical Association, 90, 429, 220-226.

CASELlA, G., e GEORGE, E.I. (1992). 'Explaining the Gibbs Sampler", The Americam Statistician, 46, 167-174.

CHIB, S. e GREENBERG, E. (1994). 'Understanding the Metropolis-Hastings algorithm". Manuscript.

CHIKKARA, R.S.; FOLKS J.L. (1989) The inverse Gaussian distribution, New York: Marcel Dekker, Inc.

CINLAR, E. (1975), Introduction To Stochastic Process, New Jersey: Prentice-Hall.

COX, D.R. e LEWIS, P.A. (1996) Statistical Analysis of the Series of Events. London: Methuen. 
DAWID, A.P. (1984). 'Statistics theory: the prequential approach". Journal of the Royal Statistics Society, Ser. A, 147, 278-292.

FORMAN, E.H.; SINGPURWALLA, N.D. (1977) "An empirical stopping rule for debugging and testing computer software", Journal American Statistics Association, 72, $360,750-757$.

FOLKS, J.L e CHHIKARA, R.S. (1978) The Inverse Gaussian distribution and its statistical application- a review. J.Roy.Stat. Soc. B40:2363-289.

GACULA, M. C., Jr., e KUBALA, J.J. (1975) Statistical Models for shelf life failures, J. Food SCI., 40:404-409.

GEISSER, S.; EDDY, W.F. (1979). A Predictive Approach to Model Selection. J. Am. Stat. Assoc., 74, 153-160.

GELFAND, A.E., DEY, D.K., e CHANG, H. (1992), 'Model Determination Using Predictive Distribution with Implementation via Sampling-Basead Methods" (with discussion), Bayesian Statistics 4, eds. J. M. Bernardo, J.O.Berger, A. P.Dawid, e A.F.M.Smith, Oxford: Oxford University Press, 147-169.

GELFAND, A.E.; SMITH, A.F.M. (1990). 'Sampling Basead Approaches to Calculating Marginal Densities". Journal of the Royal Statistics Society, Ser. A, 147, 278-292.

GEMAN, S. e GEMAN, D. (1984). 'Stochastic relaxation, Gibbs distribution and the Bayesian restorattion of images". IEEE Transactions on Pattern Analysis and Machine Intelligence, 6, 721-741.

GELMAN, A. E., e RUBIN, D.B,(1992). 'Inference from Iterative Simulation Using Multiple Sequence", Statistical Science, 7, 457-472.

GOEL, A.L.; e OKUMOTO, K. (1979) "hTime-dependent error detection rate model for software reability and other performance measures", IEEE Transaction Reability, R-28, 206-211.

GOEL, A.L. (1983) "A guidebook for software reability assesment", Technical report, RADC- TR-83-176.

GOEL, A.L. (1985). 'Software reliability models: Assumptions, limitations, and aplicability", IEEE Transactions on Software Engineering, SE-11, 1411-1423.

JEFFREYS, H. (1939). Theory of Probability. Oxford University Press.

JELINSKI, Z.; MORANDA, P (1972). 'Software reability research", Statistical Computer Performance Evaluation, Ed. W. Freiberg, 465-484. New York: Academic. 
JEWELL, W.S. (1985). 'Bayesian Extension to a Basic Model of Software Reliability", IEEE Transactions on Software Engineering, 11, 1465-1471.

JOE, H.L. (1989) 'Statistical Inference for General -Order-Statistical and Nonhomogeous-Poisson-Process Software Reliability Models," IEEE Transaction on Software Engineering, 15, 1485-1490.

JOE, H.L; REID, N. (1985). "On the software reability models of Jelinski-Moranda and Littlewood", IEEE Transaction Reliability., R-34, 3, 216-218.

KLOEK, T. VAN DIJK, H.K. (1978). 'Bayesian Extimates of Equation System Parameter: An Application of integration by Monte Carlo, Econometrika. 46, 1-19.

KUO, L. e YANG, T.Y. (1994). "Bayesian Computation for Nonhomogeneous Poisson Process in (Software) Reliability". Technical Report No .84-19, Statistics Departament, University of Connecticut.

KUO, L., LEE, J., CHOI, K. e Yang, T.Y. (1996). 'Bayesian inference for S-shaped software reliability growth models". Techincal Report 95-05, Department of Statistics, University of Connecticut.

LAWLESS, J.F.(1982). Statistical models and methods for lifetime data, New York: John Wiley \& Sons.

LITTLEWOOD, B.; ABDEL-GHALY A.A. e CHAN, P.Y. (1986) 'Evaluation of Competing Software Reliability Predictions," IEEE Transactions on Software Engineering, 12, 950-967.

LITTLEWOOD, B., e SOFER, A. (1987), "A Bayesian Modification to the JelinskiMoranda Software Reliability Growth Model", IEE/BCS Software Engineering Journal, $3,30-41$.

MAZZUCHI, T.A.; SINGPURWALLA, N.D. (1988) 'Software reliability models", in Handbook of Statistical, vol.7, Eds Krishnaiah, P,r.; Rao, C.R., Elsevier Science Publishers B.V., 73-98.

MAZZUCHI, T.A.; SOYER, R. (1988). "A Bayes empirical- Bayes model for software reliability", IEEE Transactions on reliability, R-37, 2, 248-254.

MEINHOLD, R.J. e SINGPURWALLA, N.D. (1983). 'Bayesian Analysis of a Comonly Used Model for Describing Software Failures", The Statistician, 32, 168-173.

MORANDA, P.B. (1975) 'Prediction of software reability and its applications". Proceedings of the annual reability and Maintainability Symposium, 327-332, Washington, D.C. 
MOOD, A. M., GRAYBILL F.A., BOES D.C. (1974) Introduction to the Theory of Statistics, McGraw-Hill, New York.

MUSA, J.D.; IANNINO, A.; OKUMOTO, K. (1987) Software Reliability: Measurement, Prediction, Application. New York:Wiley.

MUSA, J.D.; OKUMOTO, K. (1984) "A Logarithm poisson execution time model for software reability measurement". Proceedings of the 7th international conference on software engineering, Orlando, 230-237.

NAYLOR, J.C. e SMITH A.F.M. (1982) Application of a Method for the efficient Computation of Posterior distribution, Applied Statistics, 31, 214-225.

RAFTERY, A.E. (1987), 'Inference and Prediction for General Order Statistics Model with Unknown Population Size", Journal of the Americam Statistical Association, $82,1163-1168$.

SINGPURWALLA. N.D.; SOYER, R.(1985) "Assessing (software) reability growth using a randon coefficient autoregressive process and its ramifications", IEEE Trans. Soft. Eng., SE-11, 1456-1464.

SINGPURWALLA, N.D.; WILSON, S.P., (1994) 'Software reliability modelling”, Intern. Statis Review, 62, 3, 289-317.

TANNER, M., e WONG, W. (1987), 'The Calculation of Posterior Distribution by Data Augmentation," Journal of the Royal Statistical Society, Ser. B, 111-147.

TIERNEY, L.; KADANE, J. B. (1986). "Accurate Approximations For Posterior Moments and Marginal Densities”. J.Am. Stat. Assoc., 81, 82-86.

YAMADA, S.; OHBA, M. e OSAKI, S. (1983). 'S-shaped reliability growth modeling for software error detection". IEEE Transactions on Reliability, 32, 475-478.

YANG, T.Y. (1994). "Computational approaches to Bayesian inference for software realiability". Ph.D. Thesis, Department of Statistics, University of Connecticut, Storrs.

WEBER, C. K; LUCA, J.C. M.D.; ROCHA, A.R.C.D. (1997) "Qualidade e Produtividade em Software: Termo de Referência dos Subprograma Setorial da Qualidade e Produtividade em Software", do Programa Brasileiro da Qualidade e Produtividade PBQP, $2^{a}$ edição revisada e ampliada São Paulo: Makron Books, 1997. 
- Desconsiderar na página 22, a figura 2.2 e considerar, então, a figura abaixo:

$$
\lambda_{\mathrm{i}}=\lambda_{\mathrm{a}} \mathrm{k}_{1}^{\mathrm{i}^{\mathrm{k}}}
$$

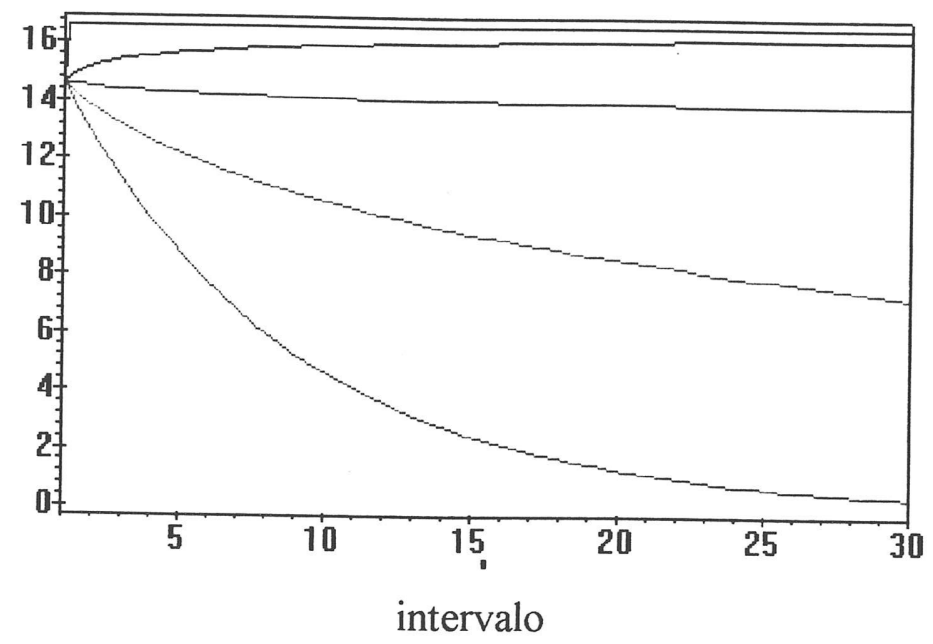

Valores de $\mathbf{k}_{2}$ :

$$
\begin{aligned}
& -\mathrm{k}_{2}=-100 \quad-\mathrm{k}_{2}=0.5 \\
& -\mathrm{k}_{2}=-20 \quad-\mathrm{k}_{2}=1.0 \\
& -\mathrm{k}_{2}=-10 \quad \mathrm{k}_{2}=10.0
\end{aligned}
$$

Figura 2.2: Taxa de falha da generalização do Modelo de Moranda (Achcar, 1995) com $\lambda_{\mathrm{a}}=16 \mathrm{e} \mathrm{k}_{1}=0.88$, para seis valores de $\mathrm{k}_{2}$.

- Substituir a expressão (2.2.2), na página 23, pela expressão abaixo:

$$
L\left(\lambda_{\mathrm{a}}, \mathrm{k}_{1}, \mathrm{k}_{2}\right)=\frac{\mathrm{e}^{-\lambda_{\mathrm{a}} \sum_{\mathrm{i}=1}^{\mathrm{n}} \mathrm{k}_{1} \mathrm{i}^{\mathrm{k}}{ }^{2}}\left(\lambda_{\mathrm{a}} \mathrm{k}_{1}^{\mathrm{i}=1} \mathrm{i}^{\mathrm{n}} \mathrm{k}_{2} \sum_{\mathrm{i}=1}^{\mathrm{n}} \mathrm{m}_{\mathrm{i}}\right.}{\prod_{\mathrm{i}=1}^{\mathrm{n}} \mathrm{m}_{\mathrm{i}} !} .
$$


- Na página 45, desconsiderar a expressão da última linha e considerar a expressão dada por:

$$
f_{\mathrm{x}_{\mathrm{n}} \backslash \mathrm{x}_{\mathrm{n}-1}}\left(\mathrm{x}_{\mathrm{n}} \backslash \mathrm{x}_{\mathrm{n}-1}\right)=\lambda\left(\mathrm{x}_{\mathrm{n}}\right) \exp \left[-\mathrm{m}\left(\mathrm{x}_{\mathrm{n}}\right)+\mathrm{m}\left(\mathrm{x}_{\mathrm{n}-1}\right)\right] \text {. }
$$

- Substituir a probabilidade não condicional, descrita na página 48 , pela expressão abaixo:

$$
\begin{aligned}
\mathrm{P}(\mathrm{M}(\mathrm{t})=\mathrm{n}) & =\sum_{\mathrm{N}=\mathrm{n}}^{\infty}\left(\begin{array}{l}
\mathrm{N} \\
\mathrm{n}
\end{array}\right) \mathrm{F}(\mathrm{t} \backslash \underset{\sim}{\sim})^{\mathrm{n}}[1-\mathrm{F}(\mathrm{t} \backslash \underset{\sim}{\sim})]^{\mathrm{N}-\mathrm{n}} \frac{\theta^{\mathrm{N}} \mathrm{e}^{-\theta}}{\mathrm{N} !} \\
& =\frac{\mathrm{e}^{-\theta \mathrm{F}(\mathrm{t} \mid \beta)}\left[\underset{\sim}{\sim}\left[\begin{array}{c}
\mathrm{t} \backslash \beta) \\
\sim
\end{array}\right]^{\mathrm{n}}\right.}{\mathrm{n} !} .
\end{aligned}
$$

- Na página 68, após o título da seção (4.2.3), considere o parágrafo:

Assumindo $k=1$, no modelo de estatísticas de ordem gama generalizada com função de valor médio definida em (4.1.10), obtemos o modelo de estatísticas de ordem Weibull (modelo de Goel, 1983).

- Desconsiderar na página 88 e 89 (Capítulo 5), os PCPOs dos modelos de estatísticas de ordem log-normal (5.2.4) e gaussiana inversa e considerar então os PCPOs:

O PCPO definido em (5.2.1), para o instante de falha futura $x_{i+1}$, dos modelos de estatísticas de ordem log-normal é dado por,

$$
\mathrm{p}\left(\mathrm{x}_{\mathrm{i}+1} \backslash \mathrm{x}_{\mathrm{i}}\right)=\iint \frac{\exp \left\{-\ln \left(\mathrm{x}_{\mathrm{i}+1}-\mu\right)^{2} / 2 \sigma^{2}\right\}}{\sqrt{2 \pi} \sigma \mathrm{x}_{\mathrm{i}+1}\left\{1-\Phi\left(\frac{\ln \mathrm{x}_{\mathrm{i}}-\mu}{\sigma}\right)\right\}} \mathrm{p}\left(\mu, \sigma \backslash \mathrm{D}_{\mathrm{x}_{\mathrm{i}}}\right) \mathrm{d} \mu \mathrm{d} \sigma .(5.2 .4)
$$

O PCPO (5.2.1) para o modelo de estatísticas de ordem gaussiana inversa é dado por, 


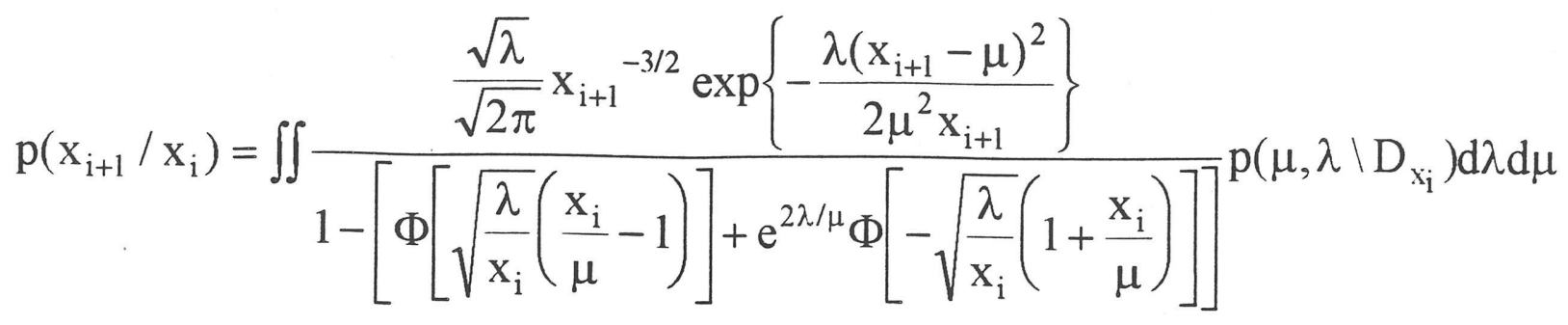

- Na página 98, inserir após a última linha, a seguinte expressão:

$$
\lambda_{\mathrm{i}}=\lambda_{\mathrm{a}} \mathrm{k}_{1}^{\mathrm{i}_{2}}
$$

- Na segunda linha, da página 101, considere a seguinte numeração das densidades condicionais de $\lambda_{\mathrm{a}}, \mathrm{k}_{1}$ e $\mathrm{k}_{2}$ :

Utilizando as amostras geradas das densidades condicionais de $\lambda_{\mathrm{a}}(3.5 .3), \mathrm{k}_{1}(3.5 .5)$ e $\mathrm{k}_{2}(3.5 .7) \ldots$

- Desconsiderar na página 131 , no último parágrafo a quarta linha e considerar, então a linha abaixo:

...razoável discrepância das estimativas Bayesianas de $\mathrm{m}\left(\mathrm{x}_{\mathrm{i}}\right)$, para o modelo de estatísticas de ordem Weibul comparado aos demais modelos. 